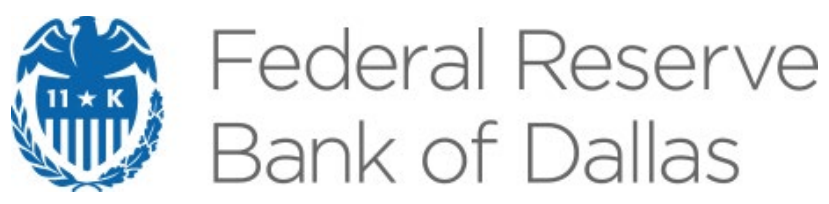

\title{
Understanding the Aggregate Effects of Credit Frictions and Uncertainty: Supplementary Materials and Additional Results
}

Nathan S. Balke, Enrique Martínez-García and Zheng Zeng

Globalization Institute Working Paper 317 Supplement

Research Department

https://doi.org/10.24149/gwp317suppr1

Working papers from the Federal Reserve Bank of Dallas are preliminary drafts circulated for professional comment. The views in this paper are those of the authors and do not necessarily reflect the views of the Federal Reserve Bank of Dallas or the Federal Reserve System. Any errors or omissions are the responsibility of the authors. 


\title{
Understanding the Aggregate Effects of Credit Frictions and Uncertainty: Supplementary Materials and Additional Results ${ }^{*}$
}

\author{
Nathan S. Balke ${ }^{\dagger}$, Enrique Martínez-García ${ }^{\ddagger}$ and Zheng Zeng ${ }^{\S}$ \\ June 12, 2017 \\ Revised: October 1, 2019
}

\begin{abstract}
Balke et al. (2017)'s model integrates financial frictions-arising from asymmetric information and costly monitoring-and time-varying uncertainty into a medium-scale Dynamic New Keynesian model. The model includes monetary policy uncertainty, financial risks (micro uncertainty), and aggregate macro-uncertainty in stochastic volatility form. In this paper, we provide the key derivations of the model as well as detailed information on our simulation and estimation approach. We use this framework to identify how uncertainty propagates and its interplay with financial frictions. We also investigate how uncertainty affects the propagation of other shocks (in particular, the propagation of TFP and monetary policy shocks).
\end{abstract}

JEL Classification: E32, E44, D8, C32

Keywords: Financial Accelerator, Stochastic Volatility, Monetary Policy Transmission, Nominal Rigidities, Perturbation Methods.

\footnotetext{
*This document has greatly benefited from valuable feedback provided by Francesco Bianchi, Andrés Blanco, Nicholas A. Bloom, Claudio Borio, Benjamin Born, William A. Brock, James Bullard, Dario Caldara, Lawrence J. Christiano, Olivier Coibion, Mario J. Crucini, Pablo Cuba-Borda, Michael B. Devereux, Martin Eichenbaum, Charles Engel, Emilio FernándezCorugedo, Andrew Filardo, Timothy S. Fuerst, Fabio Ghironi, Marc P. Giannoni, Simon Gilchrist, Pablo A. GuerrónQuintana, Joseph H. Haslag, Raju Huidrom, John Keating, Finn E. Kydland, Zheng Liu, Lilia Maliar, Rodolfo Manuelli, Antoine Martin, Leonardo Melosi, Marco del Negro, Filippo Occhino, Anna Orlik, Matthias O. Paustian, Giorgio E. Primiceri, Erwan Quintin, Barbara Rossi, Juan F. Rubío-Ramírez, Stephanie Schmitt-Grohe, Jae W. Sim, Eric Sims, Johannes Strobel, John B. Taylor, Stephen J. Terry, Martín Uribe, Víctor Valcárcel, Diego Vilán, Michael Weiss, Eric Van Wincoop, Egon Zakrajsek, and the many participants at the "Credit Market Frictions, Business Cycles, and Monetary Policy: A Research Conference in Honor of Charles Carlstrom and Timothy Fuerst" held at Notre Dame University (October 18-19, 2018). We acknowledge the excellent research assistance provided by Valerie Grossman. An earlier draft circulated under the title "Credit Uncertainty Cycles." The data and codes to replicate the results of the paper can be found here https://bit.ly/324vlkF. All remaining errors are ours alone. The views expressed here do not necessarily reflect those of the Federal Reserve Bank of Dallas or the Federal Reserve System.

${ }^{\dagger}$ Nathan S. Balke, Department of Economics, Southern Methodist University, Dallas, TX 75275, phone: (214) 768-2693, nbalke@smu.edu and Federal Reserve Bank of Dallas.

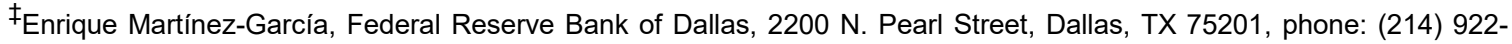
5262, fax: (214) 922-5194, emg.economics@gmail.com, webpage: https://sites.google.com/view/emgeconomics.

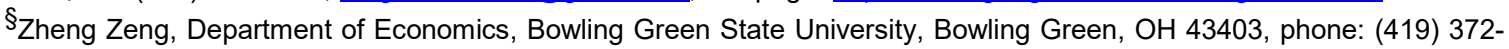
8397, zzeng@bgsu.edu.
} 


\section{Introduction}

Medium-scale DSGE models such as Christiano et al. (2005) and Smets and Wouters (2007) abstract from capital market imperfections. However, credit frictions have gained much prominence as distortions in capital markets tend to amplify and prolong over time the effects of shocks - as in the financial accelerator's adverse feedback loop that arises in the seminal work of Bernanke et al. (1999) on costly-state verification in general equilibrium. More recently, Dorofeenko et al. (2008), Christiano et al. (2014), Cesa-Bianchi and FernándezCorugedo (2018), among others, have also recognized that financial uncertainty (which we refer here as micro-uncertainty) can have first-order effects over the business cycles in the presence of credit frictions.

Financial risks or micro-uncertainty are not the only type of uncertainty that has received attention in the literature. Monetary policy uncertainty in particular and uncertainty about the state of the economy (about aggregate TFP) are among the main sources of macro/policy risk that researchers have looked at. Fernández-Villaverde et al. (2010) as well as Born and Pfeifer (2014) have explored them closely, but in economic environments which do not feature credit market imperfections like ours does. However, unlike for micro-uncertainty, their papers tend to suggest that neither aggregate (TFP) uncertainty nor policy risk appear to be of first-order importance over the business cycle.

The framework of Balke et al. (2017) employs a medium-scale Dynamic New Keynesian model with credit frictions (asymmetric information and costly monitoring as in Bernanke et al. (1999)) and uncertainty (stochastic volatility as in Fernández-Villaverde et al. (2010)). In Balke et al. (2017), we study specifically how financial frictions and micro-uncertainty affect our understanding of the monetary policy transmission mechanism through the lens of the Dynamic New Keynesian model. We also investigate the interaction between credit frictions and macro/policy and micro-uncertainty explicitly. This is where our estimated model makes its most novel contributions.

This paper complements the work of Balke et al. (2017). We describe our approach to model mean-preserving stochastic volatility in detail, provide a derivation of the optimal nominal risky debt contract between entrepreneurs (borrowers) and financial intermediaries (lenders), and include a brief description of the equilibrium conditions and steady state

equations that characterize the solution of the Balke et al. (2017) model. We also present an overview of the data used to estimate the model as well as a detailed explanation of the codes developed for the estimation and for the quantitative simulation of the pruned third-order approximation of the model's solution. Finally, this paper also contains an 
additional discussion of the main results inferred from the estimated model as well as a comprehensive set of experiments not reported in Balke et al. (2017) but conducted to establish the robustness of our findings.

\section{Modeling Mean-Preserving Stochastic Volatility}

In our framework, all shock processes with stochastic volatility - aggregate productivity (TFP), idiosyncratic productivity, and monetary policy shocks, i.e., $z_{t} \in\left\{a_{t}, \ln \left(\omega_{t}\right), m_{t}\right\}-$ can be cast in the following canonical form:

$$
\begin{aligned}
z_{t} & =\mu_{z, t}+\rho_{z}\left(z_{t-1}-\mu_{z, t-1}\right)+\sigma_{z} e^{\widehat{\sigma}_{z, t}} \varepsilon_{z, t}, \\
\mu_{z, t} & =-\frac{\left(\sigma_{z} e^{\widehat{\sigma}_{z, t}}\right)^{2}}{2}+\left(\rho_{z}\right)^{2} \mu_{z, t-1}, \\
\widehat{\sigma}_{z, t} & =v_{z} \widehat{\sigma}_{z, t-1}+\eta_{z} u_{z, t},
\end{aligned}
$$

where $\widehat{\sigma}_{z, t}=\ln \sigma_{z, t}-\ln \sigma_{z}$ and $\sigma_{z, t} \equiv \sigma_{z} e^{\widehat{\sigma}_{z, t}}$. The innovation terms $\varepsilon_{z, t}$ and $u_{z, t}$ are i.i.d. $N(0,1)$ and uncorrelated. Differences between conditional and unconditional moments of the distribution can arise under this canonical form-hence, we must note that the notion of mean-preserving spread that we adopt in this paper is that of a mean-preserving spread conditional on the history of the volatility shocks.

Any shock $z_{t} \in\left\{a_{t}, \ln \left(\omega_{t}\right), m_{t}\right\}$ is specified in logs as a stochastic (Gaussian) process, but appears in the model equilibrium conditions in levels (as $e^{z_{t}}$ ). Under the assumption of log-normality, an increase in $\sigma_{z, t}$ increases not only the variance of the shock (the dispersion for $e^{z_{t}}$ ) but also the expected mean value of $e^{z_{t}}$. Since we are interested in mean-preserving spreads that arise solely because of shifts in the dispersion of the distribution and not from indirect effects coming through the mean, we introduce a recursive correction given in (2) that reverses the conditional mean-effect of volatility on the time-varying conditional mean of the shock process $\mu_{z, t}$.

To show that this recursive correction is conditional mean-preserving, note first that $z_{t}$ and $\mu_{z, t}$ can be expanded backwards as follows:

$$
\begin{aligned}
z_{t} & =\mu_{z, t}+\sum_{i=0}^{\infty}\left(\rho_{z}\right)^{i} \sigma_{z, t-i} \varepsilon_{z, t-i} \\
\mu_{z, t} & =-\sum_{i=0}^{\infty}\left(\rho_{z}^{2}\right)^{i} \frac{\sigma_{z, t-i}^{2}}{2}
\end{aligned}
$$


When we compute the mean of the process $e^{z_{t}}$ conditional on the history of the volatility shocks, we obtain the following expression under the time-varying conditional mean $\left(\mu_{z, t}\right)$ recursion given in (2):

$$
\begin{aligned}
\mathbb{E} & {\left[e^{z_{t}} \mid \sigma_{z, t-i}, i=0, \ldots, \infty\right] } \\
& =\mathbb{E}\left[e^{\mu_{z, t}+\sum_{i=0}^{\infty}\left(\rho_{z}\right)^{i} \sigma_{z, t-i} \varepsilon_{z, t-i}} \mid \sigma_{z, t-i}, i=0, \ldots, \infty\right] \\
& =e^{\left(-\sum_{i=0}^{\infty}\left(\rho_{z}^{2}\right)^{i} \frac{\sigma_{z, t-i}^{2}}{2}\right)} \mathbb{E}\left[e^{\left(\sum_{i=0}^{\infty}\left(\rho_{z}\right)^{i} \sigma_{z, t-i} \varepsilon_{z, t-i}\right)} \mid \sigma_{z, t-i}, i=0, \ldots, \infty\right] \\
& =e^{\left(-\sum_{i=0}^{\infty}\left(\rho_{z}^{2}\right)^{i \frac{\sigma_{z, t-i}^{2}}{2}}\right)} e^{\mathbb{E}\left(\sum_{i=0}^{\infty}\left(\rho_{z}\right)^{i} \sigma_{z, t-i} \varepsilon_{z, t-i} \mid \sigma_{z, t-i}, i=0, \ldots, \infty\right)+\frac{1}{2} \mathbb{V}\left(\sum_{i=0}^{\infty}\left(\rho_{z}\right)^{i} \sigma_{z, t-i} \varepsilon_{z, t-i} \mid \sigma_{z, t-i}, i=0, \ldots, \infty\right)} \\
& =e^{\left(-\sum_{i=0}^{\infty}\left(\rho_{z}^{2}\right)^{i \frac{\sigma_{z, t-i}^{2}}{2}}\right)} e^{\left(\sum_{i=0}^{\infty}\left(\rho_{z}^{2}\right)^{i \frac{\sigma_{z, t-i}^{2}}{2}}\right)}=1
\end{aligned}
$$

which follows given that $\varepsilon_{z, t}$ are i.i.d. $N(0,1)$ innovations. As a result, this shows that the recursive correction proposed in (2) ensures that the conditional mean of the shock $z_{t}$ in levels is not affected by a change in the second moment $\sigma_{z, t}$. This is the sense in which the specification of the stochastic volatility shocks is said to be mean-preserving in our model.

Finally, we consider the implications that this mean-preserving correction has on the steady state. The standard way we characterize the deterministic steady state is: (a) to assume that $\varepsilon_{z, t}$ and $u_{z, t}$ are replaced by their unconditional means (i.e., replaced by $\mathbb{E}\left(\varepsilon_{z, t}\right)=$ $\left.\mathbb{E}\left(u_{z, t}\right)=0\right)$, and $(\mathrm{b})$ to drop the time subscript in the corresponding dynamic equations in the canonical form. Based on that logic, we get the following set of equations for the deterministic steady state:

$$
\begin{aligned}
z & =\mu_{z}=-\frac{1}{2} \frac{\sigma_{z}^{2}}{1-\rho_{z}^{2}}, \\
\widehat{\sigma}_{z} & =0
\end{aligned}
$$

for each $z \in\{a, \ln (\omega), m\}$. This describes the steady state for all shocks $z$ under our conditional mean-preserving recursive correction. 


\section{The Optimal Nominal Risky Debt Contract}

At time $t-1$, entrepreneurs purchase the aggregate stock of physical capital, $K_{t}$, which is needed for wholesale production at time $t$ at a price of $Q_{t-1}$ units of the final good per unit of physical capital. Capital-producing firms produce and sell the physical capital. The nominal expense on the acquisition of physical capital, $P_{t-1} Q_{t-1} K_{t}$, is financed with a combination of the entrepreneurs' accumulated nominal net worth (internal funds or equity), $N_{t-1}$, and external funding provided by the financial intermediaries (via one-period loans), $L_{t-1}=P_{t-1} Q_{t-1} K_{t}-N_{t-1}$. A linear technology transforms physical capital into the capital services supplied by entrepreneurs to wholesale producers at time $t$. Furthermore, these technological constraint implies that the physical capital transformed into capital services by each entrepreneur is subject to a purely idiosyncratic technology shock $\omega_{t-1}$ which is i.i.d. across entrepreneurs with $\mathbb{E}\left(\omega_{t-1}\right)=1$.

While all entrepreneurs face the same capital purchasing decision problem at $t-1$ and make identical choices, ex post differences emerge because each entrepreneur receives a different draw from $\omega_{t-1}$. At time $t$, each entrepreneur can only rent $\omega_{t-1}$ units of capital services to the wholesale producers per unit of physical capital owned. Then, in nominal terms, each entrepreneur accrues a nominal capital income of $\omega_{t-1}\left[R_{t}^{w}+P_{t} \bar{Q}_{t}(1-\delta)\right]$ at time $t$ per unit of physical capital acquired at time $t-1$. This nominal income comes from the earned competitive nominal rental rate on capital services, $R_{t}^{w}$, paid by the wholesale producers, but also from the resale value expressed in units of the final good, $\bar{Q}_{t}$, paid by the capital producers for the entrepreneurs' depreciated physical capital after production. From here it follows that each entrepreneur's nominal return accrued on physical capital is given by $\omega_{t-1} R_{t}^{e}$ and that the aggregate nominal return on capital, $R_{t}^{e}$, is defined as follows:

$$
\frac{R_{t}^{e}}{\Pi_{t}} \equiv\left[\frac{\frac{R_{t}^{w}}{P_{t}}+\bar{Q}_{t}(1-\delta)}{Q_{t-1}}\right]
$$

with $\Pi_{t} \equiv \frac{P_{t}}{P_{t-1}}$ being the gross inflation rate on final goods and $0<\delta<1$ the depreciation rate.

Given the definition of $R_{t}^{e}$ in (9), each individual entrepreneur's nominal capital income at time $t$ is $\omega_{t-1}\left[R_{t}^{w}+P_{t} \bar{Q}_{t}(1-\delta)\right] K_{t}=\omega_{t-1} R_{t}^{e} P_{t-1} Q_{t-1} K_{t}$. The idiosyncratic technology shock $\omega_{t-1}$ when realized is costlessly observed by the individual entrepreneur. However, $\omega_{t-1}$ is not observed by the financial intermediaries and verification (through monitoring) of the loan terms agreed upon is costly. The financial friction therefore arises from the agency 
costs associated with this informational asymmetry between entrepreneurs (borrowers) and financial intermediaries (lenders). ${ }^{1}$

At time $t$, default on a loan occurs whenever the nominal capital income earned after the realization of the idiosyncratic technology shock $\omega_{t-1}$ is insufficient for the entrepreneur to cover the nominal repayment of its loan, i.e., whenever

$$
\omega_{t-1} R_{t}^{e} P_{t-1} Q_{t-1} K_{t} \leq R_{t}^{L} L_{t-1}
$$

where, $R_{t}^{L}$, denotes the nominal return required at time $t$ by the financial intermediaries on the risky nominal one-period loan extended at time $t-1, L_{t-1}$. The return on the nominal one-period loan (risky debt contract) is defined implicitly in terms of a default threshold set on the idiosyncratic technology shock, $\bar{\omega}_{t-1}$, which corresponds to the draw of $\omega_{t-1}$ that equates the nominal loan repayment owed to financial intermediaries with the nominal capital income accrued by the entrepreneur-i.e., $\bar{\omega}_{t-1}$ such that $R_{t}^{L} L_{t-1}=\bar{\omega}_{t-1} R_{t}^{e} P_{t-1} Q_{t-1} K_{t}$.

Under limited liability, in case of default at time $t\left(\omega_{t-1}<\bar{\omega}_{t-1}\right)$, the financial intermediaries appropriate only the nominal capital income generated by the defaulting entrepreneur in that period - which equals $\omega_{t-1}\left[R_{t}^{w}+P_{t} \bar{Q}_{t}(1-\delta)\right] K_{t}=\omega_{t-1} R_{t}^{e} P_{t-1} Q_{t-1} K_{t}$. The financial intermediaries always choose to monitor the defaulting entrepreneurs in order to prevent them from misrepresenting the true value of $\omega_{t-1}$ and, therefore, the nominal income that can be recovered. Financial intermediaries monitor and verify the defaulting entrepreneur's income at a cost of $\mu \omega_{t-1} R_{t}^{e} P_{t-1} Q_{t-1} K_{t}$. The entrepreneur that defaults gets nothing, while the financial intermediaries are able to recover $(1-\mu) \omega_{t-1} R_{t}^{e} P_{t-1} Q_{t-1} K_{t}$. If the entrepreneur does not default $\left(\omega_{t-1} \geq \bar{\omega}_{t-1}\right)$, then he simply pays $\bar{\omega}_{t-1} R_{t}^{e} P_{t-1} Q_{t-1} K_{t}$ back to the financial intermediaries and keeps $\left(\omega_{t-1}-\bar{\omega}_{t-1}\right) R_{t}^{e} P_{t-1} Q_{t-1} K_{t}$.

Aggregate Sharing of Nominal Income from Capital. We denote the probability distribution function (pdf) and the cumulative distribution function (cdf) for the log-normallydistributed idiosyncratic technology shock $\omega_{t}$ as $\phi\left(\omega_{t} \mid \mu_{\omega, t}=-\frac{\sigma_{\omega, t}^{2}}{2}, \sigma_{\omega, t}\right)$ and $\Phi\left(\omega_{t} \mid \mu_{\omega, t}=-\frac{\sigma_{\omega, t}^{2}}{2}, \sigma_{\omega, t}\right)$, respectively. Or, to save notation, simply as $\phi\left(\omega_{t} \mid \sigma_{\omega, t}\right)$ and $\Phi\left(\omega_{t} \mid \sigma_{\omega, t}\right)$. At time $t$, the entrepreneurs anticipate the nominal capital income net of borrowing costs for each possible state of aggregate risk - where aggregate risks arise from aggregate productivity (TFP) shocks, monetary policy shocks, and from the stochastic volatility on the aggregate productivity (TFP) shock, the monetary policy shock, and the idiosyncratic technology shock —at

\footnotetext{
${ }^{1}$ The costly acquisition of information about these idiosyncratic shocks implies that financial contracts cannot be written down to completely diversify away these idiosyncratic risks. With a nominal financial contract, aggregate inflation risks can also impact the contract's borrowing terms.
} 
time $t+1$ which can be calculated as follows: ${ }^{2}$

$$
\begin{aligned}
& \int_{\bar{\omega}_{t}}^{+\infty}\left[\omega_{t} R_{t+1}^{e} P_{t} Q_{t} K_{t+1}-R_{t+1}^{L} L_{t}\right] \phi\left(\omega_{t} \mid \sigma_{\omega, t}\right) d \omega_{t} \\
& \quad=R_{t+1}^{e} P_{t} Q_{t} K_{t+1}\left[\int_{\bar{\omega}_{t}}^{+\infty}\left(\omega_{t}-\bar{\omega}_{t}\right) \phi\left(\omega_{t} \mid \sigma_{\omega, t}\right) d \omega_{t}\right]=R_{t+1}^{e} P_{t} Q_{t} K_{t+1} f\left(\bar{\omega}_{t}, \sigma_{\omega, t}\right),
\end{aligned}
$$

where

$$
f\left(\bar{\omega}_{t}, \sigma_{\omega, t}\right) \equiv \int_{\bar{\omega}_{t}}^{+\infty} \omega_{t} \phi\left(\omega_{t} \mid \sigma_{\omega, t}\right) d \omega_{t}-\bar{\omega}_{t}\left(1-\Phi\left(\bar{\omega}_{t} \mid \sigma_{\omega, t}\right)\right) .
$$

By the law of large numbers, $f\left(\bar{\omega}_{t}, \sigma_{\omega, t}\right)$ can be interpreted as the fraction of the expected nominal income from capital obtained by the entrepreneurs.

Similarly, the nominal capital income net of monitoring costs at time $t+1$ anticipated by the financial intermediaries at each possible state of aggregate risk is equal to:

$$
\begin{aligned}
& (1-\mu) \int_{0}^{\bar{\omega}_{t}}\left[\omega_{t} R_{t+1}^{e} P_{t} Q_{t} K_{t+1}\right] \phi\left(\omega_{t} \mid \sigma_{\omega, t}\right) d \omega_{t}+\int_{\bar{\omega}_{t}}^{+\infty}\left[R_{t+1}^{L} L_{t}\right] \phi\left(\omega_{t} \mid \sigma_{\omega, t}\right) d \omega_{t} \\
& =R_{t+1}^{e} P_{t} Q_{t} K_{t+1}\left[(1-\mu) \int_{0}^{\bar{\omega}_{t}} \omega_{t} \phi\left(\omega_{t} \mid \sigma_{\omega, t}\right) d \omega_{t}+\bar{\omega}_{t} \int_{\bar{\omega}_{t}}^{+\infty} \phi\left(\omega_{t} \mid \sigma_{\omega, t}\right) d \omega_{t}\right] \\
& =R_{t+1}^{e} P_{t} Q_{t} K_{t+1} g\left(\bar{\omega}_{t}, \sigma_{\omega, t}\right),
\end{aligned}
$$

where

$$
g\left(\bar{\omega}_{t}, \sigma_{\omega, t}\right) \equiv(1-\mu) \int_{0}^{\bar{\omega}_{t}} \omega_{t} \phi\left(\omega_{t} \mid \sigma_{\omega, t}\right) d \omega_{t}+\bar{\omega}_{t}\left(1-\Phi\left(\bar{\omega}_{t} \mid \sigma_{\omega, t}\right)\right) .
$$

By the law of large numbers, $g\left(\bar{\omega}_{t}, \sigma_{\omega, t}\right)$ can be interpreted as the fraction of the expected nominal capital income that accrues to the financial intermediaries.

Finally, the monitoring costs can be expressed as:

$$
\begin{aligned}
& \mu \int_{0}^{\bar{\omega}_{t}}\left[\omega_{t} R_{t+1}^{e} P_{t} Q_{t} K_{t+1}\right] \phi\left(\omega_{t} \mid \sigma_{\omega, t}\right) d \omega_{t} \\
& \quad=R_{t+1}^{e} P_{t} Q_{t} K_{t+1}\left[\mu \int_{0}^{\bar{\omega}_{t}} \omega_{t} \phi\left(\omega_{t} \mid \sigma_{\omega, t}\right) d \omega_{t}\right] \\
& \quad=R_{t+1}^{e} P_{t} Q_{t} K_{t+1} \mu G\left(\bar{\omega}_{t}, \sigma_{\omega, t}\right)
\end{aligned}
$$

\footnotetext{
${ }^{2}$ Whenever there is aggregate risk, $R_{t}^{e}$ is not known at time $t-1$ when the loan is finalized. Bernanke et al. (1999) argue that loan contracts must imply that the risk-neutral entrepreneurs bear all the aggregate risk to provide full insurance for the risk-averse households' savings loaned to them through financial intermediaries. However, loan contracts with full insurance for the savers are not necessarily optimal in more general settings (see, e.g., Hellwig (2001), Monnet and Quintin (2005), and Carlstrom et al. (2016), among others). We leave the exploration of more complex risk-sharing financial arrangements for future research.
} 
where

$$
G\left(\bar{\omega}_{t}, \sigma_{\omega, t}\right) \equiv \int_{0}^{\bar{\omega}_{t}} \omega_{t} \phi\left(\omega_{t} \mid \sigma_{\omega, t}\right) d \omega_{t}
$$

By the law of large numbers, $\mu G\left(\bar{\omega}_{t}, \sigma_{\omega, t}\right)$ can be interpreted as the fraction of the expected nominal capital income that is lost due to monitoring costs. Then, naturally, a nominal capital income sharing rule between entrepreneurs and financial intermediaries arises in the following form:

$$
f\left(\bar{\omega}_{t}, \sigma_{\omega, t}\right)+g\left(\bar{\omega}_{t}, \sigma_{\omega, t}\right)=1-\mu G\left(\bar{\omega}_{t}, \sigma_{\omega, t}\right),
$$

where $\mu G\left(\bar{\omega}_{t}, \sigma_{\omega, t}\right) \geq 0$ determines the nominal capital income losses due to monitoring costs which would be set to zero only if monitoring costs are zero, i.e., if $\mu=0$.

The Loan Contracting Problem. With the information available at time $t$, the entrepreneurs' expected nominal capital income net of borrowing costs implied by equation (11) is:

$$
P_{t} Q_{t} K_{t+1} \mathbb{E}_{t}\left[R_{t+1}^{e}\right] f\left(\bar{\omega}_{t}, \sigma_{\omega, t}\right)
$$

Similarly, financial intermediaries' expected nominal capital income net of monitoring costs given by equation (13) is:

$$
P_{t} Q_{t} K_{t+1} \mathbb{E}_{t}\left[R_{t+1}^{e}\right] g\left(\bar{\omega}_{t}, \sigma_{\omega, t}\right)
$$

The formal contracting problem, at time $t$, reduces to choosing the quantity of physical capital, $K_{t+1}$, and the default threshold, $\bar{\omega}_{t}$, that maximize the entrepreneurs' expected nominal capital income net of borrowing costs given by (18) subject to the following participation constraint for the financial intermediaries:

$$
P_{t} Q_{t} K_{t+1} \mathbb{E}_{t}\left[R_{t+1}^{e}\right]\left[1-f\left(\bar{\omega}_{t}, \sigma_{\omega, t}\right)-\mu G\left(\bar{\omega}_{t}, \sigma_{\omega, t}\right)\right] \geq I_{t} L_{t}=I_{t}\left[P_{t} Q_{t} K_{t+1}-N_{t}\right]
$$

where the left-hand side combines the expected nominal capital income for the financial intermediaries in (19) with the sharing rule equation in (17). The equality on the right-hand side of (20) follows from the aggregate balance sheet equation of the entrepreneurs (i.e., from $\left.P_{t} Q_{t} K_{t+1}=N_{t}+L_{t}\right)$. All financial intermediaries share equally in the pool of loans to entrepreneurs. If lenders participate in this loan contract, they always supply the desired nominal loans, $L_{t}$, as long as they accrue a rate of return on their loan portfolio greater than or equal to the return owed on deposits (the nominal risk-free interest rate, $I_{t}$ ). In other words, we do not explicitly consider here the possibility of credit rationing. 
It follows from the first-order condition with respect to $\bar{\omega}_{t}$ that:

$$
-f_{\omega}\left(\bar{\omega}_{t}, \sigma_{\omega, t}\right)+\lambda\left(\bar{\omega}_{t}, \sigma_{\omega, t}\right)\left[f_{\omega}\left(\bar{\omega}_{t}, \sigma_{\omega, t}\right)+\mu G_{\omega}\left(\bar{\omega}_{t}, \sigma_{\omega, t}\right)\right]=0
$$

where $\lambda\left(\bar{\omega}_{t}, \sigma_{\omega, t}\right)$ is the Lagrange multiplier on the financial intermediaries' participation constraint in (20). Here, we define $f_{\omega}\left(\bar{\omega}_{t}, \sigma_{\omega, t}\right) \equiv \frac{\partial f\left(\bar{\omega}_{t}, \sigma_{\omega, t}\right)}{\partial \bar{\omega}_{t}}$ and $G_{\omega}\left(\bar{\omega}_{t}, \sigma_{\omega, t}\right) \equiv \frac{\partial G\left(\bar{\omega}_{t}, \sigma_{\omega, t}\right)}{\partial \bar{\omega}_{t}}$. By virtue of this optimality condition, we say that the shadow cost of enticing the participation of the financial intermediaries in this loan contract is given by:

$$
\lambda\left(\bar{\omega}_{t}, \sigma_{\omega, t}\right)=\frac{f_{\omega}\left(\bar{\omega}_{t}, \sigma_{\omega, t}\right)}{f_{\omega}\left(\bar{\omega}_{t}, \sigma_{\omega, t}\right)+\mu G_{\omega}\left(\bar{\omega}_{t}, \sigma_{\omega, t}\right)} .
$$

The Kuhn-Tucker conditions imply that the complementary slackness condition

$$
\lambda\left(\bar{\omega}_{t}, \sigma_{\omega, t}\right)\left[I_{t}\left(P_{t} Q_{t} K_{t+1}-N_{t}\right)-P_{t} Q_{t} K_{t+1} \mathbb{E}_{t}\left[R_{t+1}^{e}\right]\left(1-f\left(\bar{\omega}_{t}, \sigma_{\omega, t}\right)-\mu G\left(\bar{\omega}_{t}, \sigma_{\omega, t}\right)\right)\right]=0,
$$

and $\lambda\left(\bar{\omega}_{t}, \sigma_{\omega, t}\right) \geq 0$ hold. This together with (22) implies that the participation constraint must always be binding since the Lagrange multiplier is non-zero.

The binding participation constraint can be re-written as:

$$
\frac{P_{t} Q_{t} K_{t+1}}{N_{t}} \mathbb{E}_{t}\left(\frac{R_{t+1}^{e}}{I_{t}}\right)\left(1-f\left(\bar{\omega}_{t}, \sigma_{\omega, t}\right)-\mu G\left(\bar{\omega}_{t}, \sigma_{\omega, t}\right)\right)=\left[\frac{P_{t} Q_{t} K_{t+1}}{N_{t}}-1\right],
$$

or, more compactly, as

$$
\frac{P_{t} Q_{t} K_{t+1}}{N_{t}}=\frac{1}{1-\mathbb{E}_{t}\left(\frac{R_{t+1}^{e}}{I_{t}}\right)\left(\frac{\Psi\left(\bar{\omega}_{t}, \sigma_{\omega, t}\right)-f\left(\bar{\omega}_{t}, \sigma_{\omega, t}\right)}{\lambda\left(\bar{\omega}_{t}, \sigma_{\omega, t}\right)}\right)},
$$

where $\Psi\left(\bar{\omega}_{t}, \sigma_{\omega, t}\right)$ is

$$
\begin{aligned}
\Psi\left(\bar{\omega}_{t}, \sigma_{\omega, t}\right) & \equiv f\left(\bar{\omega}_{t}, \sigma_{\omega, t}\right)+\lambda\left(\bar{\omega}_{t}, \sigma_{\omega, t}\right)\left(1-f\left(\bar{\omega}_{t}, \sigma_{\omega, t}\right)-\mu G\left(\bar{\omega}_{t}, \sigma_{\omega, t}\right)\right) \\
& =f\left(\bar{\omega}_{t}, \sigma_{\omega, t}\right)+\lambda\left(\bar{\omega}_{t}, \sigma_{\omega, t}\right) g\left(\bar{\omega}_{t}, \sigma_{\omega, t}\right)
\end{aligned}
$$

given the nominal capital income sharing rule in (17). From (25) and (26), it follows that the optimal leverage can be expressed as

$$
\frac{P_{t} Q_{t} K_{t+1}}{N_{t}}=\frac{1}{1-\mathbb{E}_{t}\left(\frac{R_{t+1}^{e}}{I_{t}}\right) g\left(\bar{\omega}_{t}, \sigma_{\omega, t}\right)},
$$


which depends on the expected aggregate excess return as given by $\mathbb{E}_{t}\left(\frac{R_{t+1}^{e}}{I_{t}}\right)$ and the fraction of the expected nominal capital income that accrues to the financial intermediaries $g\left(\bar{\omega}_{t}, \sigma_{\omega, t}\right)$.

The loan contracting problem also requires the following first-order condition with respect to physical capital, $K_{t+1}$, to hold:

$$
\mathbb{E}_{t}\left(\frac{R_{t+1}^{e}}{I_{t}}\right) \Psi\left(\bar{\omega}_{t}, \sigma_{\omega, t}\right)-\lambda\left(\bar{\omega}_{t}, \sigma_{\omega, t}\right)=0
$$

where we implicitly conjecture that $\bar{\omega}_{t}$ is conditioned only on variables known at time $t$ (to be more precise, we conjecture that $\bar{\omega}_{t}$ is conditioned on the pair $\left.\left(\frac{P_{t} Q_{t} K_{t+1}}{N_{t}}, \sigma_{\omega, t}\right)\right){ }^{3}$ Simply re-arranging this expression gives us that,

$$
\mathbb{E}_{t}\left(\frac{R_{t+1}^{e}}{I_{t}}\right)=\frac{\lambda\left(\bar{\omega}_{t}, \sigma_{\omega, t}\right)}{\Psi\left(\bar{\omega}_{t}, \sigma_{\omega, t}\right)}
$$

This optimality condition determines the excess returns per unit of physical capital above the nominal interest rate, $I_{t}$, that are required to make the loan contract worthwhile to both entrepreneurs and financial intermediaries.

If we combine equations (25) and (29), then it follows that:

$$
\begin{aligned}
\frac{P_{t} Q_{t} K_{t+1}}{N_{t}} & =\frac{1}{1-\frac{\lambda\left(\bar{\omega}_{t}, \sigma_{\omega, t}\right)}{\Psi\left(\bar{\omega}_{t}, \sigma_{\omega, t}\right)}\left(\frac{\Psi\left(\bar{\omega}_{t}, \sigma_{\omega, t}\right)-f\left(\bar{\omega}_{t}, \sigma_{\omega, t}\right)}{\lambda\left(\bar{\omega}_{t}, \sigma_{\omega, t}\right)}\right)} \\
& =\frac{1}{1-\left(\frac{\Psi\left(\bar{\omega}_{t}, \sigma_{\omega, t}\right)-f\left(\bar{\omega}_{t}, \sigma_{\omega, t}\right)}{\Psi\left(\bar{\omega}_{t}, \sigma_{\omega, t}\right)}\right)}=\frac{\Psi\left(\bar{\omega}_{t}, \sigma_{\omega, t}\right)}{f\left(\bar{\omega}_{t}, \sigma_{\omega, t}\right)}
\end{aligned}
$$

which validates our conjecture that the default threshold $\bar{\omega}_{t}$ is a function of observables known at time $t$, that is, our conjecture that $\bar{\omega}_{t} \equiv \bar{\omega}\left(\frac{P_{t} Q_{t} K_{t+1}}{N_{t}}, \sigma_{\omega, t}\right)$. Given the relationships derived in (29) and (30), the specification of the credit risk spread can be re-expressed as follows:

$$
\mathbb{E}_{t}\left[\frac{R_{t+1}^{e}}{I_{t}}\right]=s\left(\frac{P_{t} Q_{t} K_{t+1}}{N_{t}}, \sigma_{\omega, t}\right),
$$

where

$$
s\left(\frac{P_{t} Q_{t} K_{t+1}}{N_{t}}, \sigma_{\omega, t}\right) \equiv \frac{\lambda\left(\bar{\omega}\left(\frac{P_{t} Q_{t} K_{t+1}}{N_{t}}, \sigma_{\omega, t}\right), \sigma_{\omega, t}\right)}{\Psi\left(\bar{\omega}\left(\frac{P_{t} Q_{t} K_{t+1}}{N_{t}}, \sigma_{\omega, t}\right), \sigma_{\omega, t}\right)}
$$

\footnotetext{
${ }^{3}$ The micro-uncertainty shock, $\sigma_{\omega, t}$, and the asset-to-net-worth ratio, $\frac{P_{t} Q_{t} K_{t+1}}{N_{t}}$, are thought to be known by all agents at time $t$ when the loan contract is signed.
} 
or simply as

$$
\mathbb{E}_{t}\left[R_{t+1}^{e}\right]=s\left(\frac{P_{t} Q_{t} K_{t+1}}{N_{t}}, \sigma_{\omega, t}\right) I_{t},
$$

given that $I_{t}$ is known at time $t$ and can be taken out of the expectation. This characterization of the credit risk spread (or external finance premium) expands the Bernanke et al. (1999) financial accelerator framework by modeling loan contracts explicitly in nominal terms and by linking the external finance premium itself to the micro-uncertainty shocks, $\sigma_{\omega, t}$.

Loan Contract Terms Under Log-Normality. The idiosyncratic technology shock $\omega_{t}$ is $\log$-normally distributed, i.e., $\ln \left(\omega_{t}\right) \sim N\left(\mu_{\omega, t}, \sigma_{\omega, t}^{2}\right)$. The conditional variance, $\sigma_{\omega, t}^{2}$, reflects the time $t$ dispersion of the cross-sectional distribution of the entrepreneurs' idiosyncratic productivity. We set $\sigma_{\omega, t} \equiv \sigma_{\omega} e^{\widehat{\sigma}_{\omega, t}}$ and allow the conditional variance $\widehat{\sigma}_{\omega, t} \equiv \ln \sigma_{\omega, t}-\ln \sigma_{\omega}$ to be time-varying as follows:

$$
\widehat{\sigma}_{\omega, t}=v_{\omega} \widehat{\sigma}_{\omega, t-1}+\eta_{\omega} u_{\omega, t}
$$

where $u_{\omega, t}$ is i.i.d. $N(0,1)$. We refer to this as the exogenous micro-uncertainty shock. The parameter $0<v_{\omega}<1$ determines the persistence of the idiosyncratic technology shock's $\log$-volatility $\widehat{\sigma}_{\omega, t}$, the unconditional expected volatility is given by $\sigma_{\omega}>0$, and the standard deviation of its innovations by $\eta_{\omega} \geq 0$. By setting the time-varying conditional mean $\mu_{\omega, t}$ to be $\mu_{\omega, t}=-\frac{\sigma_{\omega, t}^{2}}{2}$, we ensure the unconditional mean of the idiosyncratic productivity shock $\omega_{t}$ to be mean-preserving (i.e., we ensure $\mathbb{E}\left(\omega_{t}\right)=1$ ) isolating the effects of pure second moment shocks (micro-uncertainty) from first moment or level effects of the shock.

The density function of the log-normally-distributed idiosyncratic technology shock $\omega_{t}$ is

$$
\phi\left(\omega_{t} \mid \mu_{\omega, t}, \sigma_{\omega, t}\right)=\frac{1}{\omega_{t} \sigma_{\omega, t} \sqrt[2]{2 \pi}} e^{-\frac{\left(\ln \omega_{t}-\mu_{\omega, t}\right)^{2}}{2 \sigma_{\omega, t}^{2}}}, \omega_{t}>0
$$

and its cumulative distribution function is

$$
\begin{gathered}
\Phi\left(\omega_{t} \mid \mu_{\omega, t}, \sigma_{\omega, t}\right)=\operatorname{Pr}\left(\omega_{t} \leq \omega \mid \mu_{\omega, t}, \sigma_{\omega, t}^{2}\right)=\frac{1}{2}\left[1+\operatorname{erf}\left(\frac{\ln \omega_{t}-\mu_{\omega, t}}{\sigma_{\omega, t} \sqrt[2]{2}}\right)\right] \\
\quad \operatorname{erf}\left(\frac{\ln \omega_{t}-\mu_{\omega, t}}{\sigma_{\omega, t} \sqrt[2]{2}}\right) \equiv \frac{2}{\sqrt[2]{\pi}} \int_{0}^{\frac{\ln \omega_{t}-\mu_{\omega, t}}{\sigma_{\omega, t} \sqrt[2]{2}}} e^{-x^{2}} d x
\end{gathered}
$$

where erf $(\cdot)$ denotes the Gaussian error function. 
Given the log-normal assumption in $(35)-(37)$, we obtain that: ${ }^{4}$

$$
\begin{aligned}
\int_{\bar{\omega}_{t}}^{+\infty} \omega_{t} \phi\left(\omega_{t} \mid \sigma_{\omega, t}\right) d \omega_{t} & =\int_{\bar{\omega}_{t}}^{+\infty} \frac{1}{\sigma_{\omega, t} \sqrt[2]{2 \pi}} e^{-\frac{\left(\ln \omega_{t}-\mu_{\omega, t}\right)^{2}}{2 \sigma_{\omega, t}^{2}}} d \omega_{t} \\
& =\left(-\left.\frac{1}{2} e^{\mu_{\omega, t}+\frac{\sigma_{\omega, t}^{2}}{2}} \operatorname{erf}\left(\frac{\mu_{\omega, t}+\sigma_{\omega, t}^{2}-\ln \omega_{t}}{\sigma_{\omega, t} \sqrt[2]{2}}\right)\right|_{\bar{\omega}_{t}} ^{+\infty}\right) \\
& =\left(-\frac{1}{2} e^{\mu_{\omega, t}+\frac{\sigma_{\omega, t}^{2}}{2}} \operatorname{erf}(-\infty)\right)-\left(-\frac{1}{2} e^{\mu_{\omega, t}+\frac{\sigma_{\omega, t}^{2}}{2}} \operatorname{erf}\left(\frac{\mu_{\omega, t}+\sigma_{\omega, t}^{2}-\ln \bar{\omega}_{t}}{\sigma_{\omega, t} \sqrt[2]{2}}\right)\right) \\
& =\frac{1}{2} e^{\mu_{\omega, t}+\frac{\sigma_{\omega, t}^{2}}{2}}\left[1+\operatorname{erf}\left(\frac{\mu_{\omega, t}+\sigma_{\omega, t}^{2}-\ln \bar{\omega}_{t}}{\sigma_{\omega, t} \sqrt[2]{2}}\right)\right]
\end{aligned}
$$

and

$$
\Phi\left(\bar{\omega}_{t} \mid \mu_{\omega, t}, \sigma_{\omega, t}\right)=\frac{1}{2}\left[1+\operatorname{erf}\left(\frac{\ln \bar{\omega}_{t}-\mu_{\omega, t}}{\sigma_{\omega, t} \sqrt[2]{2}}\right)\right] .
$$

Hence, it is possible to characterize the share $f\left(\bar{\omega}_{t}, \sigma_{\omega, t}\right)$ in $(12)$ as:

$$
f\left(\bar{\omega}_{t}, \sigma_{\omega, t}\right)=\frac{1}{2} e^{\mu_{\omega, t}+\frac{\sigma_{\omega, t}^{2}}{2}}\left[1+\operatorname{erf}\left(\frac{\mu_{\omega, t}+\sigma_{\omega, t}^{2}-\ln \bar{\omega}_{t}}{\sigma_{\omega, t} \sqrt[2]{2}}\right)\right]-\bar{\omega}_{t}\left(1-\Phi\left(\bar{\omega}_{t} \mid \sigma_{\omega, t}\right)\right)
$$

in terms of the error function erf $(\cdot)$.

Similarly, given the log-normal assumption in $(35)-(37)$, the function $G\left(\bar{\omega}_{t}, \sigma_{\omega, t}\right)$ can be re-expressed as:

$$
\begin{aligned}
G\left(\bar{\omega}_{t}, \sigma_{\omega, t}\right) & \equiv \int_{0}^{\bar{\omega}_{t}} \omega_{t} \phi\left(\omega_{t} \mid \sigma_{\omega, t}\right) d \omega_{t} \\
& =\int_{0}^{+\infty} \omega_{t} \phi\left(\omega_{t} \mid \sigma_{\omega, t}\right) d \omega_{t}-\int_{\bar{\omega}_{t}}^{+\infty} \omega_{t} \phi\left(\omega_{t} \mid \sigma_{\omega, t}\right) d \omega_{t}
\end{aligned}
$$

where $\mathbb{E}\left(\omega_{t}\right)=\int_{0}^{+\infty} \omega_{t} \phi\left(\omega_{t} \mid \sigma_{\omega, t}\right) d \omega_{t}=e^{\mu_{\omega, t}+\frac{\sigma_{\omega, t}^{2}}{2}}$. Hence, the functional form for $G\left(\bar{\omega}_{t}, \sigma_{\omega, t}\right)$ becomes:

$$
\begin{aligned}
G\left(\bar{\omega}_{t}, \sigma_{\omega, t}\right) & =1-\int_{\bar{\omega}_{t}}^{+\infty} \omega_{t} \phi\left(\omega_{t} \mid \sigma_{\omega, t}\right) d \omega_{t}=1-\int_{\bar{\omega}_{t}}^{+\infty} \frac{1}{\sigma_{\omega, t} \sqrt[2]{2 \pi}} e^{-\frac{\left(\ln \omega_{t}-\mu_{\omega, t}\right)^{2}}{2 \sigma_{\omega, t}^{2}}} d \omega_{t} \\
& =1-\frac{1}{2} e^{\mu_{\omega, t}+\frac{\sigma_{\omega, t}^{2}}{2}}\left[1+\operatorname{erf}\left(\frac{\mu_{\omega, t}+\sigma_{\omega, t}^{2}-\ln \bar{\omega}_{t}}{\sigma_{\omega, t} \sqrt[2]{2}}\right)\right],
\end{aligned}
$$

\footnotetext{
${ }^{4}$ The properties of the error function erf $(\cdot)$ imply that $\lim _{x \rightarrow-\infty} \operatorname{erf}(x)=-1$.
} 
expressed in terms of the error function $\operatorname{erf}(\cdot)$. The functional forms for $f\left(\bar{\omega}_{t}, \sigma_{\omega, t}\right)$ and for $G\left(\bar{\omega}_{t}, \sigma_{\omega, t}\right)$ together with equation (17) completely characterize the split of the nominal capital income between entrepreneurs and financial intermediaries, while also accounting for the losses due to costly monitoring. As indicated before, here we assume a mean-preserving distribution that ensures the unconditional mean is equal to one in every period (i.e., $\mathbb{E}\left(\omega_{t}\right)=$ 1) by setting $\mu_{\omega, t}=-\frac{\sigma_{\omega, t}^{2}}{2}$.

Given the functional form $f\left(\bar{\omega}_{t}, \sigma_{\omega, t}\right)$ derived in (39)-(40), we can compute the derivative of $f\left(\bar{\omega}_{t}, \sigma_{\omega, t}\right)$ with respect to $\bar{\omega}_{t}$ as:

$$
\begin{aligned}
f\left(\bar{\omega}_{t}, \sigma_{\omega, t}\right) & =\frac{1}{2} e^{\mu_{\omega, t}+\frac{\sigma_{\omega, t}^{2}}{2}}\left[1+\operatorname{erf}\left(\frac{\mu_{\omega, t}+\sigma_{\omega, t}^{2}-\ln \bar{\omega}_{t}}{\sigma_{\omega, t} \sqrt[2]{2}}\right)\right]-\bar{\omega}_{t}\left(1-\frac{1}{2}\left[1+\operatorname{erf}\left(\frac{\ln \bar{\omega}_{t}-\mu_{\omega, t}}{\sigma_{\omega, t} \sqrt[2]{2}}\right)\right]\right) \\
f_{\omega}\left(\bar{\omega}_{t}, \sigma_{\omega, t}\right) & \equiv \frac{\partial f\left(\bar{\omega}_{t}, \sigma_{\omega, t}\right)}{\partial \bar{\omega}_{t}}=-\frac{1}{2}\left[1+\operatorname{erf}\left(\frac{\mu_{\omega, t}-\ln \left(\bar{\omega}_{t}\right)}{\sigma_{\omega, t} \sqrt[2]{2}}\right)\right]
\end{aligned}
$$

Similarly, we can compute the derivative of $G\left(\bar{\omega}_{t}, \sigma_{\omega, t}\right)$ with respect to $\bar{\omega}_{t}$ as:

$$
\begin{aligned}
G\left(\bar{\omega}_{t}, \sigma_{\omega, t}\right) & =1-\frac{1}{2} e^{\mu_{\omega, t}+\frac{\sigma_{\omega, t}^{2}}{2}}\left[1+\operatorname{erf}\left(\frac{\mu_{\omega, t}+\sigma_{\omega, t}^{2}-\ln \bar{\omega}_{t}}{\sigma_{\omega, t} \sqrt[2]{2}}\right)\right] \\
G_{\omega}\left(\bar{\omega}_{t}, \sigma_{\omega, t}\right) & \equiv \frac{\partial G\left(\bar{\omega}_{t}, \sigma_{\omega, t}\right)}{\partial \bar{\omega}_{t}}=0.398942\left(\frac{\left(\bar{\omega}_{t}\right)^{\frac{\mu_{\omega, t}}{\sigma_{\omega, t}}}}{\sigma_{\omega, t}}\right) e^{-\left(\frac{\left(\mu_{\omega, t}\right)^{2}+\left(\ln \left(\bar{\omega}_{t}\right)\right)^{2}}{2 \sigma_{\omega, t}^{2}}\right)} .
\end{aligned}
$$

Finally, the Lagrange multiplier $\lambda\left(\bar{\omega}_{t}, \sigma_{\omega, t}\right)=\frac{f_{\omega}\left(\bar{\omega}_{t}, \sigma_{\omega, t}\right)}{f_{\omega}\left(\bar{\omega}_{t}, \sigma_{\omega, t}\right)+\mu G_{\omega}\left(\bar{\omega}_{t}, \sigma_{\omega, t}\right)}$ can be expressed as:

$$
\lambda\left(\bar{\omega}_{t}, \sigma_{\omega, t}\right)=\frac{-\frac{1}{2}\left[1+\operatorname{erf}\left(\frac{\mu_{\omega, t}-\ln \left(\bar{\omega}_{t}\right)}{\sigma_{\omega, t} \sqrt[2]{2}}\right)\right]}{-\frac{1}{2}\left[1+\operatorname{erf}\left(\frac{\mu_{\omega, t}-\ln \left(\bar{\omega}_{t}\right)}{\sigma_{\omega, t} \sqrt[2]{2}}\right)\right]+\mu\left(0.398942\left(\frac{\left(\bar{\omega}_{t}\right)^{\frac{\mu_{\omega, t}}{\sigma_{\omega, t}^{2}}}}{\sigma_{\omega, t}^{2}}\right) e^{-\left(\frac{\left(\mu_{\omega, t}\right)^{2}+\left(\ln \left(\bar{\omega}_{t}\right)\right)^{2}}{2 \sigma_{\omega, t}^{2}}\right)}\right.} .
$$

In this case, it follows that $-1<f_{\omega}\left(\bar{\omega}_{t}, \sigma_{\omega, t}\right)<0$ and $G_{\omega}\left(\bar{\omega}_{t}, \sigma_{\omega, t}\right)>0$. From (22) and the Kuhn-Tucker condition $\lambda\left(\bar{\omega}_{t}, \sigma_{\omega, t}\right) \geq 0$, it follows that $\lambda\left(\bar{\omega}_{t}, \sigma_{\omega, t}\right)=\frac{f_{\omega}\left(\bar{\omega}_{t}, \sigma_{\omega, t}\right)}{f_{\omega}\left(\bar{\omega}_{t}, \sigma_{\omega, t}\right)+\mu G_{\omega}\left(\bar{\omega}_{t}, \sigma_{\omega, t}\right)} \geq 0$ and this, in turn, can only hold if $f_{\omega}\left(\bar{\omega}_{t}, \sigma_{\omega, t}\right)+\mu G_{\omega}\left(\bar{\omega}_{t}, \sigma_{\omega, t}\right)<0$. Moreover, given that $G_{\omega}\left(\bar{\omega}_{t}, \sigma_{\omega, t}\right)>0$, we can go further and show that $\lambda\left(\bar{\omega}_{t}, \sigma_{\omega, t}\right) \geq 1$ when the parameter that governs the fraction of nominal capital income lost due to monitoring costs $\mu$ lies within the unit interval. 
The Borrowing Costs' Channel. Under perfect information and costless monitoring, the risk-neutral entrepreneurs are willing to invest on an additional unit of physical capital if $\mathbb{E}_{t}\left[R_{t+1}^{e}\right] \geq I_{t}$ where $I_{t}$ is the nominal risk-free interest rate and $R_{t}^{e}$ the entrepreneurs' aggregate nominal return on physical capital. $I_{t}$ represents the cost of the loanable funds obtained from households' deposits by the financial intermediaries and, therefore, the opportunity cost of capital investment. If $\mathbb{E}_{t}\left[R_{t+1}^{e}\right]>I_{t}$, then the entrepreneurs' demand for funds is infinite. Hence, competitive market forces would imply the following arbitrage condition $\mathbb{E}_{t}\left[R_{t+1}^{e}\right]=I_{t}$. Moreover, under costless monitoring, credit frictions are removed and the Modigliani-Miller theorem holds in effect (capital investment decisions are independent of the capital structure, i.e., of whether entrepreneurs are equity or debt financed).

In our model, private information and costly monitoring with limited liability are the key assumptions needed to incorporate credit frictions and pin down the balance sheet structure of entrepreneurs (a key departure from the Modigliani-Miller theorem on the indeterminacy of the capital structure). The costly-state verification framework we propose also has the advantage that it endogenizes the probability of default, unlike what occurs with other models of credit distortions such as those based on borrowing constraints (Kiyotaki and Moore (1997)). Private information implies that only entrepreneurs can costlessly observe their own returns, while lenders must pay a fixed fraction of the realized nominal capital income to cover the costs of monitoring and verification of the defaulting entrepreneurs. Limited liability on the part of the entrepreneurs, in turn, introduces a lower bound (of zero) on the minimum payoff that the entrepreneurs can accrue.

In this costly-state verification setting, we end up with a modified efficiency condition to determine the optimal choice of physical capital in the model implied by equation (32) (33). If we look back at (29), given the definition of $\Psi\left(\bar{\omega}_{t}, \sigma_{\omega, t}\right)$ in (26), we can infer that $\frac{\lambda\left(\bar{\omega}_{t}, \sigma_{\omega, t}\right)}{\Psi\left(\bar{\omega}_{t}, \sigma_{\omega, t}\right)}=\frac{\lambda\left(\bar{\omega}_{t}, \sigma_{\omega, t}\right)}{f\left(\bar{\omega}_{t}, \sigma_{\omega, t}\right)+\lambda\left(\bar{\omega}_{t}, \sigma_{\omega, t}\right) g\left(\bar{\omega}_{t}, \sigma_{\omega, t}\right)} \geq \lambda\left(\bar{\omega}_{t}, \sigma_{\omega, t}\right) \geq 1$ since we have already shown before that $\lambda\left(\bar{\omega}_{t}, \sigma_{\omega, t}\right) \geq 1$. And, in the special case where monitoring becomes costless $(\mu=0)$, we immediately get that $\frac{\lambda\left(\bar{\omega}_{t}, \sigma_{\omega, t}\right)}{\Psi\left(\bar{\omega}_{t}, \sigma_{\omega, t}\right)}=1$. Hence, this proves the following proposition:

Proposition 1 In equilibrium, (32) - (33) implies that $\mathbb{E}_{t}\left(R_{t+1}^{e}\right)=\frac{\lambda\left(\bar{\omega}_{t}, \sigma_{\omega, t}\right)}{\Psi\left(\bar{\omega}_{t}, \sigma_{\omega, t}\right)} I_{t} \geq I_{t}$ which holds with equality only if $\mu=0$. In other words, the external borrowing costs (endogenous credit risk spread) faced by entrepreneurs must be at or above the nominal risk-free rate, $I_{t}$, when monitoring is costly $(\mu>0)$.

We show here that $\mathbb{E}_{t}\left(R_{t+1}^{e}\right)>I_{t}$ if the monitoring cost parameter satisfies that $0<\mu<1$ 
(and $\mathbb{E}_{t}\left(R_{t+1}^{e}\right)=I_{t}$ if $\mu=0$ ). Inverting the efficiency condition in (33), we obtain that

$$
\frac{P_{t} Q_{t} K_{t+1}}{N_{t}}=\phi\left(\mathbb{E}_{t}\left(\frac{R_{t+1}^{e}}{I_{t}}\right), \sigma_{\omega, t}\right)
$$

where $\phi\left(\mathbb{E}_{t}\left(\frac{R_{t+1}^{e}}{I_{t}}\right), \sigma_{\omega, t}\right) \equiv s^{-1}\left(\mathbb{E}_{t}\left(\frac{R_{t+1}^{e}}{I_{t}}\right), \sigma_{\omega, t}\right)$ is the optimal leverage (asset-to-equity ratio) and $s^{-1}\left(\cdot, \sigma_{\omega, t}\right)$ is the inverse of the credit spread $s\left(\frac{P_{t} Q_{t} K_{t+1}}{N_{t}}, \sigma_{\omega, t-1}\right)$ in (32). In equilibrium, the credit spread is inversely related to the net-worth-to-asset ratio (or equity ratio) of the entrepreneurs, $\frac{N_{t}}{P_{t} Q_{t} K_{t+1}}$, and directly related to micro-uncertainty, $\sigma_{\omega, t}$. We view $\sigma_{\omega, t}$ as a measure of the dispersion of the idiosyncratic shock $\omega_{t}$ and, accordingly, consider the consequences of a mean-preserving increases in the credit spread.

From here, the demand for physical capital in nominal terms can be expressed as follows:

$$
P_{t} Q_{t} K_{t+1}=\phi\left(\mathbb{E}_{t}\left(\frac{R_{t+1}^{e}}{I_{t}}\right), \sigma_{\omega, t}\right) N_{t}
$$

with $\frac{\partial \phi\left(\mathbb{E}_{t}\left(\frac{R_{t+1}^{e}}{I_{t}}\right), \sigma_{\omega, t}\right)}{\partial \mathbb{E}_{t}\left(\frac{R_{t+1}^{e}}{I_{t}}\right)}>0$ and $\frac{\partial \phi\left(\mathbb{E}_{t}\left(\frac{R_{t+1}^{e}}{I_{t}}\right), \sigma_{\omega, t}\right)}{\partial \sigma_{\omega, t}}<0$. Hence, increasing the idiosyncratic risk or reducing the excess returns for entrepreneurs over the risk-free rate reduces capital demand by tightening the margins and reducing the optimal leverage ratio required. This is the heart of the mechanism that we explore quantitatively and empirically in the Balke et al. (2017) model. Our theory motivates these (credit) risk shocks based on the idea of uncertainty about the idiosyncratic shocks - or micro-uncertainty - that cannot be fully insured against due to asymmetries of information between borrowers and lenders.

The leverage ratio moves with asset values (Tobin's q) - particularly on impact, as the stock of capital and net worth are slower to adjust - and influences capital demand as a result of a real options effect (from fixed adjustment costs on capital) or through a precautionary savings effect (from risk aversion). The macro-uncertainty and monetary policy uncertainty shocks only impact the credit spreads and the demand for capital to the extent that they feed through the endogenous leverage ratio. In turn, the demand for capital depends inversely on the uncertainty attached to the idiosyncratic shocks (direct effects of micro-uncertainty). And, what is most significant, the endogenous propagation of other shocks through the financial accelerator mechanism described here also depends on whether idiosyncratic shocks are more or less uncertain. In other words, the propagation of shocks is conditional on the amount of micro-uncertainty in the economy through this financial lever mechanism.

We find that whenever micro-uncertainty $\sigma_{\omega, t}$ is low, the optimal leverage is high and- 
concurrently - the credit risk spreads are low. In that environment, conventional monetary policy shocks would have a more muted impact on the capital demand resulting in smaller amplification effects on investment and economic activity. However, this also implies a higher level of capital. That is to say, we find that lower uncertainty about the idiosyncratic shocks results in higher leverage (lessening the precautionary motive) and also in higher capital accumulation. In turn, TFP level shocks are amplified given that the lower credit spreads and more benign financial conditions make it possible for entrepreneurs to take advantage more fully of the changes in aggregate productivity (TFP).

\section{Benchmark Model Equilibrium Conditions}

In the presence of habit preferences it follows that:

$$
\left(C_{t}-b C_{t-1}\right)^{-\chi}-b \beta \mathbb{E}_{t}\left[\left(C_{t+1}-b C_{t}\right)^{-\chi}\right]=\Lambda_{t}
$$

where $\Lambda_{t}$ is the Lagrange multiplier on the household's budget constraint expressed in units of the final good. ${ }^{5}$

\footnotetext{
${ }^{5}$ The local curvature of the household's preferences on consumption (the Arrow-Pratt coefficient of relative risk aversion) is given by$$
-\frac{C_{t}\left(\frac{\partial \Lambda_{t}}{\partial C_{t}}\right)}{\Lambda_{t}}=\left\{\begin{array}{l}
\frac{\chi}{\frac{\left(C_{t}-b C_{t-1}\right)^{-\chi}-b \beta \mathbb{E}_{t}\left[\left(C_{t+1}-b C_{t}\right)^{-\chi}\right]}{C_{t}\left[\left(C_{t}-b C_{t-1}\right)^{-\chi-1}+b^{2} \beta \mathbb{E}_{t}\left(\left(C_{t+1}-b C_{t}\right)^{-\chi-1}\right)\right]}} \text { if } 0<b \leq 1, \\
\chi \text { if } b=0,
\end{array}\right.
$$

while the corresponding Arrow-Pratt coefficient of relative prudence is given by

$$
-\frac{\left(\frac{\partial^{2} \Lambda_{t}}{\partial C_{t} \partial C_{t}}\right)}{\left(\frac{\partial \Lambda_{t}}{\partial C_{t}}\right)}=\left\{\begin{array}{l}
\frac{1+\chi}{\frac{\left(C_{t}-b C_{t-1}\right)^{-\chi-1}+b^{2} \beta \mathbb{\Xi}_{t}\left(\left(C_{t+1}-b C_{t}\right)^{-\chi-1}\right)}{C_{t}\left[\left(C_{t}-b C_{t-1}\right)^{-\chi-2}-b^{3} \beta \mathbb{\Xi}_{t}\left(\left(C_{t+1}-b C_{t}\right)^{-\chi-2}\right)\right]}} \text { if } 0<b \leq 1, \\
1+\chi \text { if } b=0 .
\end{array}\right.
$$

Local risk aversion on consumption refers to the curvature of the utility function, whereas local prudence refers to the curvature of the marginal utility function (the Lagrange multiplier $\Lambda_{t}$ ). If the marginal utility is convex (i.e., if the Lagrange multiplier $\Lambda_{t}$ satisfies that $\left(\frac{\partial^{2} \Lambda_{t}}{\partial C_{t}^{2}}\right)>0$ ), then households are "prudent" and, faced with higher uncertainty, may consume less and work more in order to self-insure against future shocks (a precautionary savings motive). When households have no internal habits $(b=0)$, their preferences feature precautionary savings but their relative prudence is constant and depends solely on the relative risk aversion parameter $\chi \geq 0$. When households have internal habits $(0<b \leq 1)$, their relative prudence depends not solely on the relative risk aversion parameter $\chi \geq 0$ but also on the internal habit persistence parameter $0<b \leq 1$. Thus a given relative risk aversion parameter $\chi$ can mean a time-varying relative risk aversion and risk prudence. As a result, the strength of the precautionary savings motive will vary as households become more or less prudent as a function of the consumption and internal habit paths.
} 


\subsection{Goods Market}

Equilibrium in the final goods market means the production of the final good $Y_{t}$ in each period $t$ is allocated either to households' consumption, $C_{t}$, and capital producers' investment, $X_{t}$, or gets lost due to price adjustment costs in the retail sector, $\frac{\varphi_{p}}{2}\left(\Pi_{t}-1\right)^{2} Y_{t}$, and to agency costs in the financial intermediation sector, $\mu G\left(\bar{\omega}_{t-1}, \sigma_{\omega, t-1}\right) \frac{R_{t}^{e}}{\Pi_{t}} Q_{t-1} K_{t}$. Hence, the resource constraint can be expressed as: ${ }^{6}$

$$
\left[1-\frac{\varphi_{p}}{2}\left(\Pi_{t}-1\right)^{2}\right] Y_{t}=C_{t}+X_{t}+\mu G\left(\bar{\omega}_{t-1}, \sigma_{\omega, t-1}\right) \frac{R_{t}^{e}}{\Pi_{t}} Q_{t-1} K_{t}
$$

In the symmetric equilibrium that characterizes the pricing decisions of retailers, final goods, $Y_{t}$, are equal to wholesale output produced with an aggregate Cobb-Douglas production function that uses labor from households, $H_{t}$, labor from entrepreneurs, $H_{t}^{e}$, and rented capital services from entrepreneurs, $K_{t}$, as inputs: ${ }^{7}$

$$
Y_{t}=e^{\widehat{a}_{t}}\left(K_{t}\right)^{\alpha}\left(H_{t}^{e}\right)^{\vartheta}\left(H_{t}\right)^{1-\alpha-\vartheta}
$$

where the TFP process in logs is given as $\widehat{a}_{t} \equiv a_{t}-a$ with $a=-\frac{1}{2} \frac{\sigma_{a}^{2}}{1-\left(\rho_{a}\right)^{2}}$.

The optimizing household's Euler equation can be written as:

$$
1=\beta \mathbb{E}_{t}\left[\left(\frac{\Lambda_{t+1}}{\Lambda_{t}}\right)\left(\frac{I_{t}}{\Pi_{t+1}}\right)\right]
$$

where $\Lambda_{t}$ is the Lagrange multiplier on the household's budget constraint expressed in units of the final good.

The optimizing firms' price-setting dynamics (the Phillips curve) in the symmetric equi-

\footnotetext{
${ }^{6}$ The entrepreneurial consumption $C_{t}^{e}$ in $(62)$ is the fraction of final output accumulated through entrepreneurial net worth that corresponds to the dying entrepreneurs and hence does not detract from current production of final goods (which explains why it does not appear in the resource constraint in (49)).

${ }^{7}$ Capital services are homogenous in production and, accordingly, perfectly substitutable across entrepreneurs. Given that in equilibrium the aggregate supply of capital services must equate the aggregate stock of physical capital owned by the entrepreneurs, we use the same notation $K_{t}$ interchangeably for physical capital and capital services. Furthermore, in equilibrium, each identical wholesale producer's demand for capital services is the same and therefore must be equal to the aggregate stock physical capital $K_{t}$.
} 
librium becomes: ${ }^{8}$

$$
\left(1-P_{t}^{w r}\right) \epsilon=\left[1-\varphi_{p}\left(\Pi_{t}-1\right) \Pi_{t}\right]+\varphi_{p} \beta \mathbb{E}_{t}\left[\left(\frac{\Lambda_{t+1}}{\Lambda_{t}}\right)\left(\left(\Pi_{t+1}-1\right) \Pi_{t+1} \frac{Y_{t+1}}{Y_{t}}\right)\right]
$$

where the gross inflation rate on final goods is given as $\Pi_{t} \equiv \frac{P_{t}}{P_{t-1}}$, and the inverse of $P_{t}^{w r} \equiv \frac{P_{t}^{w}}{P_{t}}$ can be interpreted as the gross markup of each retail variety over the wholesale good's price. ${ }^{9}$

\subsection{Labor Market}

We define households' real wages as $W_{t}^{r} \equiv \frac{W_{t}}{P_{t}}$ and entrepreneurial real wages as $W_{t}^{e r} \equiv \frac{W_{t}^{e}}{P_{t}}$. Hence, the labor demand equations can be expressed as:

$$
\begin{gathered}
W_{t}^{r}=(1-\alpha-\vartheta) \frac{P_{t}^{w r} Y_{t}}{H_{t}} \\
W_{t}^{e r}=\vartheta \frac{P_{t}^{w r} Y_{t}}{H_{t}^{e}}
\end{gathered}
$$

while the labor supply equations are give by:

$$
\begin{gathered}
H_{t}^{e}=1 \\
W_{t}^{r}=\frac{\kappa H_{t}^{\xi}}{\Lambda_{t}}
\end{gathered}
$$

\subsection{Capital Market}

We define the aggregate nominal return on capital as $R_{t}^{e}$ and the real rental rate on capital in units of the final good paid by the wholesale producers to the entrepreneurs for the capital

\footnotetext{
${ }^{8}$ Retailers change nominal prices every period but face a Rotemberg (1982) quadratic adjustment cost function $s_{p}\left(P_{t}(j), P_{t-1}(j)\right)$ given by: ${ }_{p}\left(P_{t}(j), P_{t-1}(j)\right)=\frac{\varphi_{p}}{2}\left(\frac{P_{t}(j)}{P_{t-1}(j)}-1\right)^{2}, \forall j \in[0,1]$, where $\varphi_{p} \geq 0$ scales the quadratic price adjustment cost. For their problem to be well-defined, it must hold that $\frac{\varphi_{p}}{2}\left(\pi_{t}-1\right)^{2}<1$, i.e., $\pi_{t} \in\left(1-\sqrt[2]{\frac{2}{\varphi_{p}}}, 1+\sqrt[2]{\frac{2}{\varphi_{p}}}\right)$.

${ }^{9}$ Absent nominal price adjustment costs $\left(\varphi_{p}=0\right)$, the gross markup $\frac{1}{P_{t}^{w r}}=\frac{\epsilon}{\epsilon-1}$ under monopolistic competition and flexible prices is a time-invariant function of the elasticity of substitution across variaties $\epsilon>1$. With nominal price adjustment costs $\left(\varphi_{p}>0\right)$, the gross markup implied by (52) becomes timevarying and dependent on macroeconomic factors (not just on the parameter $\epsilon$ ).
} 
services provided as $R_{t}^{w r} \equiv \frac{R_{t}^{w}}{P_{t}}$, i.e.,

$$
\frac{R_{t}^{e}}{\Pi_{t}}=\left[\frac{R_{t}^{w r}+\bar{Q}_{t}(1-\delta)}{Q_{t-1}}\right]
$$

where the rental rate on capital services is given by the marginal product of capital in the production of wholesale goods:

$$
R_{t}^{w r}=\alpha \frac{P_{t}^{w r} Y_{t}}{K_{t}}
$$

The optimization problem of the capital goods producers pins down the relative price of physical capital or Tobin's q, $Q_{t}$, as:

$$
Q_{t}=\left(\frac{\frac{X_{t}}{K_{t}}}{\delta}\right)^{\frac{1}{\varphi_{k}}}
$$

The relative resale value of capital $\bar{Q}_{t}$ is a function of Tobin's q, $Q_{t}$, and the investmentto-capital ratio, $\frac{X_{t}}{K_{t}}$, implied by the zero-profit condition for capital goods producers: ${ }^{10}$

$$
Q_{t}\left(\frac{(\delta)^{\frac{1}{\varphi_{k}}}\left(\frac{X_{t}}{K_{t}}\right)^{1-\frac{1}{\varphi_{k}}}-\frac{1}{\varphi_{k}} \delta}{1-\frac{1}{\varphi_{k}}}\right)-\frac{X_{t}}{K_{t}}-(1-\delta)\left(\bar{Q}_{t}-Q_{t}\right)=0
$$

We denote the law of motion for next period's physical capital, $K_{t+1}$, under the techno-

\footnotetext{
${ }^{10}$ Time-variation in the relative price of capital $Q_{t}$ serves as an additional amplification and propagation mechanism in the model. The difference between $\bar{Q}_{t}$ and $Q_{t}$ implied by (60) is of second-order importance and, accordingly, omitted by Bernanke et al. (1999) and Martínez-García (2014), which rely on a first-order perturbation solution. Unlike them, we take explicit account of the difference between $\bar{Q}_{t}$ and $Q_{t}$ in our set-up, as we solve our model up to a higher order of approximation. This matters in particular for the key definition of aggregate nominal returns on capital, $R_{t}^{e}$, in (57).
} 
logical constraints of these capital-producing firms as: ${ }^{11}$

$$
K_{t+1}=(1-\delta) K_{t}+\left(\frac{(\delta)^{\frac{1}{\varphi_{k}}}\left(\frac{X_{t}}{K_{t}}\right)^{1-\frac{1}{\varphi_{k}}}-\frac{1}{\varphi_{k}} \delta}{1-\frac{1}{\varphi_{k}}}\right) K_{t}
$$

From the entrepreneurs' optimization problem, we also obtain two equations that pin down the entrepreneurial consumption, $C_{t}^{e}$, and the law of motion for entrepreneurial net worth adjusted by inflation, $\frac{N_{t}}{P_{t}}$ :

$$
\begin{gathered}
C_{t}^{e}=(1-\gamma) \Psi\left(\bar{\omega}_{t-1}, \sigma_{\omega, t-1}\right) \frac{R_{t}^{e}}{\Pi_{t}} N_{t-1}^{r} \\
N_{t}^{r}=W_{t}^{e r} H_{t}^{e}+\gamma \Psi\left(\bar{\omega}_{t-1}, \sigma_{\omega, t-1}\right) \frac{R_{t}^{e}}{\Pi_{t}} N_{t-1}^{r}
\end{gathered}
$$

where $N_{t}^{r} \equiv \frac{N_{t}}{P_{t}}$ denotes the real net worth of the entrepreneurs in units of the final good while $N_{t}$ is the nominal net worth. Entrepreneurs are risk-neutral and die with probability $1-\gamma$ each period, so equation (62) reflects the fact that under such conditions entrepreneurs would postpone their consumption until death at which time they "eat" the entire net worth they have accumulated during their lifetime. ${ }^{12}$

${ }^{11}$ The adjustment cost function adopted here is $s_{k}\left(\frac{X_{t}}{K_{t}}\right)=\left(\frac{\delta}{1-\frac{1}{\varphi_{k}}}\right)\left[\left(\frac{\frac{X_{t}}{K_{t}}}{\delta}\right)^{1-\frac{1}{\varphi_{k}}}-\frac{1}{\varphi_{k}}\right]$. The adjustment cost function $s_{k}\left(\frac{X_{t}}{K_{t}}\right)$ satisfies that $s_{k}(\delta)=\delta$ and $s_{k}^{\prime}(\delta)=1$ ensuring that adjustment costs drop out in steady state. The adjustment cost function $s_{k}\left(\frac{X_{t}}{K_{t}}\right)$ also implies that $s_{k}(\cdot)>0$ if $\frac{X_{t}}{K_{t}} \geq \delta\left(\frac{1}{\varphi_{k}}\right)^{\frac{\varphi_{k}}{\varphi_{k}-1}}$ and $\varphi_{k}>1$, while $s_{k}^{\prime}(\cdot)>0$ and $s_{k}^{\prime \prime}(\cdot)<0$ for any $\frac{X_{t}}{K_{t}} \geq 0$ and any $\varphi_{k}>0$. The range of the parameter space for $\varphi_{k}$ that appears more plausible is given by $\varphi_{k}>1$ and, in this case, $0<\delta\left(\frac{1}{\varphi_{k}}\right)^{\frac{\varphi_{k}}{\varphi_{k}-1}}<\delta$ implies that $\frac{X_{t}}{K_{t}} \geq \delta$ suffices (but is not necessary) to ensure $s_{k}(\cdot)>0$. We interpret the adjustment cost function as penalizing underinvestment when investment falls significantly below the steady state replacement rate given by the depreciation rate $\delta$. In other words, when $X_{t}<\delta K_{t}$ it follows that $0<s_{k}\left(\frac{X_{t}}{K_{t}}\right) K_{t}<\delta K_{t}$ and, subject to the law of motion for physical capital in (61), that $K_{t+1}<K_{t}$. If investment is sufficiently low $\left(X_{t}<\delta\left(\frac{1}{\varphi_{k}}\right)^{\frac{\varphi_{k}}{\varphi_{k}-1}} K_{t}\right)$ then it holds that $s_{k}\left(\frac{X_{t}}{K_{t}}\right) K_{t}<0<\delta K_{t}$ and $K_{t+1}<(1-\delta) K_{t}$.

${ }^{12}$ The exogenous death makes entrepreneurs more impatient than households and induces them to be borrowers and households to be savers. It also prevents entrepreneurs from accumulating infinite wealth and becoming self-financing. The risk-neutrality of the entrepreneurs leads them to postpone consumption until death as long as the gross growth rate of nominal net worth $N_{t}$ is above the inverse of the discount factor $\gamma \beta$ as that would afford them a higher present-discounted consumption and lifetime utility than if they tried to anticipate some consumption. 


\subsection{Credit Market}

The first-order conditions that solve the optimal risky debt contract described in Section 3 are:

$$
\begin{aligned}
& \Phi\left(\bar{\omega}_{t} \mid \mu_{\omega, t}, \sigma_{\omega, t}\right)=\frac{1}{2}\left[1+\operatorname{erf}\left(\frac{\ln \bar{\omega}_{t}-\mu_{\omega, t}}{\sigma_{\omega} e^{\widehat{\sigma}_{\omega, t}} \sqrt[2]{2}}\right)\right] \\
& f\left(\bar{\omega}_{t}, \sigma_{\omega, t}\right)=\frac{1}{2} e^{\mu_{\omega, t}+\frac{\left(\sigma_{\omega} e^{\widehat{\sigma} \omega, t}\right)^{2}}{2}}\left[1+\operatorname{erf}\left(\frac{\mu_{\omega, t}+\left(\sigma_{\omega} e^{\widehat{\sigma}_{\omega, t}}\right)^{2}-\ln \bar{\omega}_{t}}{\sigma_{\omega} e^{\widehat{\sigma}_{\omega, t}} \sqrt[2]{2}}\right)\right]-\bar{\omega}_{t}\left(1-\Phi\left(\bar{\omega}_{t} \mid \sigma_{\omega, t}\right)\right) \\
& G\left(\bar{\omega}_{t}, \sigma_{\omega, t}\right)=1-\frac{1}{2} e^{\mu_{\omega, t}+\frac{\left(\sigma_{\omega} e^{\widehat{\sigma} \omega, t}\right)^{2}}{2}}\left[1+\operatorname{erf}\left(\frac{\mu_{\omega, t}+\left(\sigma_{\omega} e^{\widehat{\sigma}_{\omega, t}}\right)^{2}-\ln \bar{\omega}_{t}}{\sigma_{\omega} e^{\widehat{\sigma}_{\omega, t}} \sqrt[2]{2}}\right)\right] \\
& f\left(\bar{\omega}_{t}, \sigma_{\omega, t}\right)+g\left(\bar{\omega}_{t}, \sigma_{\omega, t}\right)=1-\mu G\left(\bar{\omega}_{t}, \sigma_{\omega, t}\right) \\
& \lambda\left(\bar{\omega}_{t}, \sigma_{\omega, t}\right)\left(f_{\omega}\left(\bar{\omega}_{t}, \sigma_{\omega, t}\right)+\mu G_{\omega}\left(\bar{\omega}_{t}, \sigma_{\omega, t}\right)\right)=f_{\omega}\left(\bar{\omega}_{t}, \sigma_{\omega, t}\right) \\
& f_{\omega}\left(\bar{\omega}_{t}, \sigma_{\omega, t}\right)=-\frac{1}{2}\left[1+\operatorname{erf}\left(\frac{\mu_{\omega, t}-\ln \left(\bar{\omega}_{t}\right)}{\sigma_{\omega} e^{\widehat{\sigma}_{\omega, t}} \sqrt[2]{2}}\right)\right] \\
& G_{\omega}\left(\bar{\omega}_{t}, \sigma_{\omega, t}\right)=0.398942\left(\frac{\left(\bar{\omega}_{t}\right)^{\frac{\mu_{\omega, t}}{\left(\sigma_{\omega} e^{\widehat{\sigma} \omega, t}\right)^{2}}}}{\sigma_{\omega} e^{\widehat{\sigma}_{\omega, t}}}\right) e^{-\left(\frac{\left(\mu_{\omega, t}\right)^{2}+\left(\ln \left(\bar{\omega}_{t}\right)\right)^{2}}{2\left(\sigma_{\omega} e^{\widehat{\sigma}_{\omega}, t}\right)^{2}}\right)} \\
& \Psi\left(\bar{\omega}_{t}, \sigma_{\omega, t}\right)=f\left(\bar{\omega}_{t}, \sigma_{\omega, t}\right)+\lambda\left(\bar{\omega}_{t}, \sigma_{\omega, t}\right) g\left(\bar{\omega}_{t}, \sigma_{\omega, t}\right) \\
& \frac{Q_{t} K_{t+1}}{N_{t}^{r}}=\frac{\Psi\left(\bar{\omega}_{t}, \sigma_{\omega, t}\right)}{f\left(\bar{\omega}_{t}, \sigma_{\omega, t}\right)} \\
& \mathbb{E}_{t}\left(R_{t+1}^{e}\right)=\frac{\lambda\left(\bar{\omega}_{t}, \sigma_{\omega, t}\right)}{\Psi\left(\bar{\omega}_{t}, \sigma_{\omega, t}\right)} I_{t}
\end{aligned}
$$

where the stochastic volatility of the idiosyncratic technology shock $\sigma_{\omega, t}$ is computed as $\sigma_{\omega, t}=\sigma_{\omega} e^{\widehat{\sigma}_{\omega, t}}$

\subsection{Monetary Policy}

The monetary authority sets the nominal interest rate, $I_{t}$, following a modified Taylor (1993)type monetary policy rule with inertia which we express in index form as:

$$
\frac{I_{t}}{I}=\left(\frac{I_{t-1}}{I}\right)^{\rho_{i}}\left(\left(\frac{\Pi_{t}}{\Pi_{t}^{*}}\right)^{\psi_{\pi}}\left(\frac{Y_{t}}{Y_{t-1}}\right)^{\psi_{x}}\right)^{1-\rho_{i}} e^{\widehat{m}_{t}}
$$


where $\widehat{m}_{t} \equiv m_{t}-m$ and $m=-\frac{1}{2} \frac{\sigma_{m}^{2}}{1-\left(\rho_{m}\right)^{2}}$. We also impose that the monetary authority targets zero net-inflation in every period, i.e., $\Pi_{t}^{*}=1$.

\subsection{Exogenous Shocks with Stochastic Volatility}

Denoting $\widehat{\sigma}_{a, t} \equiv \ln \sigma_{a, t}-\ln \sigma_{a}, \widehat{\sigma}_{\omega, t} \equiv \ln \sigma_{\omega, t}-\ln \sigma_{\omega}$ and $\widehat{\sigma}_{m, t} \equiv \ln \sigma_{m, t}-\ln \sigma_{m}$, we define the shock processes as follows:

\section{Aggregate Productivity (TFP) Shock (with Macro-Uncertainty)}

$$
\begin{gathered}
\mu_{a, t}=-\frac{\left(\sigma_{a} e^{\widehat{\sigma}_{a, t}}\right)^{2}}{2}+\left(\rho_{a}\right)^{2} \mu_{a, t-1} \\
a_{t}=\mu_{a, t}+\rho_{a}\left(a_{t-1}-\mu_{a, t-1}\right)+\sigma_{a} e^{\widehat{\sigma}_{a, t}} \varepsilon_{a, t} \\
\widehat{\sigma}_{a, t}=v_{a} \widehat{\sigma}_{a, t-1}+\eta_{a} u_{a, t} \\
A_{t} \equiv e^{\widehat{a}_{t}} \text { (auxiliary equation) }
\end{gathered}
$$

where the stochastic volatility of the TFP shock $\sigma_{a, t}$ is computed as $\sigma_{a, t}=\sigma_{a} e^{\widehat{\sigma}_{a, t}}$.

\section{Micro-Uncertainty Shock}

$$
\begin{gathered}
\mu_{\omega, t}=-\frac{\left(\sigma_{\omega} e^{\widehat{\sigma}_{\omega, t}}\right)^{2}}{2} \\
\widehat{\sigma}_{\omega, t}=v_{\omega} \widehat{\sigma}_{\omega, t-1}+\eta_{\omega} u_{\omega, t} \\
O_{t} \equiv \mathbb{E}\left(\omega_{t}\right)=e^{\mu_{\omega, t}+\frac{\left(\sigma_{\omega} e^{\widehat{\sigma}_{\omega, t}}\right)^{2}}{2}} \text { (auxiliary equation) }
\end{gathered}
$$

where the stochastic volatility of the idiosyncratic technology shock $\sigma_{\omega, t}$ is computed as $\sigma_{\omega, t}=\sigma_{\omega} e^{\widehat{\sigma}_{\omega, t}}$

\section{Monetary Policy Shock (with Policy-Uncertainty)}

$$
\begin{gathered}
\mu_{m, t}=-\frac{\left(\sigma_{m} e^{\widehat{\sigma}_{m, t}}\right)^{2}}{2}+\left(\rho_{m}\right)^{2} \mu_{m, t-1} \\
m_{t}=\mu_{m, t}+\rho_{m}\left(m_{t-1}-\mu_{m, t-1}\right)+\sigma_{m} e^{\widehat{\sigma}_{m, t}} \varepsilon_{m, t} \\
\widehat{\sigma}_{m, t}=v_{m} \widehat{\sigma}_{m, t-1}+\eta_{m} u_{m, t}
\end{gathered}
$$




$$
M_{t} \simeq 1+\widehat{m}_{t}(\text { auxiliary equation })
$$

where the stochastic volatility of the monetary policy shock $\sigma_{m, t}$ is computed as $\sigma_{m, t}=$ $\sigma_{m} e^{\widehat{\sigma}_{m, t}}$.

\section{Deterministic Steady State}

\subsection{Habit Preferences}

$$
(1-b \beta)(1-b)^{-\chi} C^{-\chi}=\Lambda
$$

\subsection{Goods Market}

$$
\begin{gathered}
{\left[1-\frac{\varphi_{p}}{2}(\Pi-1)^{2}\right] Y=C+X+\mu G\left(\bar{\omega}, \sigma_{\omega}\right) \frac{R^{e}}{\Pi} Q K} \\
Y=e^{\widehat{a}}(K)^{\alpha}\left(H^{e}\right)^{\vartheta}(H)^{1-\alpha-\vartheta} \\
1=\beta\left(\frac{I}{\Pi}\right) \\
\left(1-P^{w r}\right) \epsilon=1-(1-\beta) \varphi_{p}(\Pi-1) \Pi
\end{gathered}
$$

where $\widehat{a}=0$.

\subsection{Labor Market}

$$
\begin{gathered}
W^{r}=(1-\alpha-\vartheta) \frac{P^{w r} Y}{H} \\
W^{e r}=\vartheta \frac{P^{w r} Y}{H^{e}} \\
H^{e}=1 \\
W^{r}=\frac{\kappa H^{\xi}}{\Lambda}
\end{gathered}
$$

\subsection{Capital Market}

$$
Q=\left(\frac{\frac{X}{K}}{\delta}\right)^{\frac{1}{\varphi_{k}}}
$$




$$
\begin{gathered}
Q\left(\frac{(\delta)^{\frac{1}{\varphi_{k}}}\left(\frac{X}{K}\right)^{1-\frac{1}{\varphi_{k}}}-\frac{1}{\varphi_{k}} \delta}{1-\frac{1}{\varphi_{k}}}\right)-\frac{X}{K}-(1-\delta)(\bar{Q}-Q)=0 \\
\frac{R^{e}}{\Pi}=\left[\frac{R^{w r}+\bar{Q}(1-\delta)}{Q}\right] \\
R^{w r}=\alpha \frac{P^{w r} Y}{K} \\
K=(1-\delta) K+\left(\frac{(\delta)^{\frac{1}{\varphi_{k}}}\left(\frac{X}{K}\right)^{1-\frac{1}{\varphi_{k}}}-\frac{1}{\varphi_{k}} \delta}{1-\frac{1}{\varphi_{k}}}\right) K \\
C^{e}=(1-\gamma) \Psi\left(\bar{\omega}, \sigma_{\omega}\right) \frac{R^{e}}{\Pi} N^{r} \\
N^{r}=W^{e r} H^{e}+\gamma \Psi\left(\bar{\omega}, \sigma_{\omega}\right) \frac{R^{e}}{\Pi} N^{r}
\end{gathered}
$$

\subsection{Credit Market}

$$
\begin{aligned}
& \Phi\left(\bar{\omega} \mid \mu_{\omega}, \sigma_{\omega}\right)=\frac{1}{2}\left[1+\operatorname{erf}\left(\frac{\ln \bar{\omega}-\mu_{\omega}}{\sigma_{\omega} e^{\widehat{\sigma}_{\omega}} \sqrt[2]{2}}\right)\right] \\
& f\left(\bar{\omega}, \sigma_{\omega}\right)=\frac{1}{2} e^{\mu_{\omega}+\frac{\left(\sigma_{\omega} e^{\widehat{\sigma} \omega}\right)^{2}}{2}}\left[1+\operatorname{erf}\left(\frac{\mu_{\omega}+\left(\sigma_{\omega} e^{\widehat{\sigma}_{\omega}}\right)^{2}-\ln \bar{\omega}}{\sigma_{\omega} e^{\widehat{\sigma}_{\omega}} \sqrt[2]{2}}\right)\right]-\bar{\omega}\left(1-\Phi\left(\bar{\omega} \mid \sigma_{\omega}\right)\right) \\
& G\left(\bar{\omega}, \sigma_{\omega}\right)=1-\frac{1}{2} e^{\mu_{\omega}+\frac{\left(\sigma_{\omega} e^{\widehat{\sigma}_{\omega}}\right)^{2}}{2}}\left[1+\operatorname{erf}\left(\frac{\mu_{\omega}+\left(\sigma_{\omega} e^{\widehat{\sigma}_{\omega}}\right)^{2}-\ln \bar{\omega}}{\sigma_{\omega} e^{\widehat{\sigma}_{\omega}} \sqrt[2]{2}}\right)\right] \\
& f\left(\bar{\omega}, \sigma_{\omega}\right)+g\left(\bar{\omega}, \sigma_{\omega}\right)=1-\mu G\left(\bar{\omega}, \sigma_{\omega}\right) \\
& \lambda\left(\bar{\omega}, \sigma_{\omega}\right)\left(f_{\omega}\left(\bar{\omega}, \sigma_{\omega}\right)+\mu G_{\omega}\left(\bar{\omega}, \sigma_{\omega}\right)\right)=f_{\omega}\left(\bar{\omega}, \sigma_{\omega}\right) \\
& f_{\omega}\left(\bar{\omega}, \sigma_{\omega}\right)=-\frac{1}{2}\left[1+\operatorname{erf}\left(\frac{\mu_{\omega}-\ln (\bar{\omega})}{\sigma_{\omega} \sqrt[2]{2}}\right)\right] \\
& G_{\omega}\left(\bar{\omega}, \sigma_{\omega}\right)=0.398942\left(\frac{(\bar{\omega})^{\frac{\mu_{\omega}}{\left(\sigma_{\omega} e^{\widehat{\sigma} \omega}\right)^{2}}}}{\left(\sigma_{\omega} e^{\widehat{\sigma}_{\omega}}\right)}\right) e^{-\left(\frac{\left(\mu_{\omega}\right)^{2}+(\ln (\bar{\omega}))^{2}}{2\left(\sigma_{\omega} e^{\widehat{\sigma}_{\omega}}\right)^{2}}\right)} \\
& \Psi\left(\bar{\omega}, \sigma_{\omega}\right)=f\left(\bar{\omega}, \sigma_{\omega}\right)+\lambda\left(\bar{\omega}, \sigma_{\omega}\right) g\left(\bar{\omega}, \sigma_{\omega}\right) \\
& \frac{Q K}{N^{r}}=\frac{\Psi\left(\bar{\omega}, \sigma_{\omega}\right)}{f\left(\bar{\omega}, \sigma_{\omega}\right)}
\end{aligned}
$$




$$
R^{e}=\frac{\lambda\left(\bar{\omega}, \sigma_{\omega}\right)}{\Psi\left(\bar{\omega}, \sigma_{\omega}\right)} I
$$

\subsection{Monetary Policy Rule}

$$
\frac{I}{I}=\left(\frac{I}{I}\right)^{\rho_{i}}\left(\left(\frac{\Pi}{\Pi^{*}}\right)^{\psi_{\pi}}\left(\frac{Y}{Y}\right)^{\psi_{x}}\right)^{1-\rho_{i}} e^{\widehat{m}}
$$

where $\widehat{m}=0$.

\subsection{Exogenous Shocks with Stochastic Volatility}

Aggregate Productivity (TFP) Shock (with Macro-Uncertainty)

$$
\begin{gathered}
\mu_{a}=-\frac{\left(\sigma_{a} e^{\widehat{\sigma}_{a}}\right)^{2}}{2}+\left(\rho_{a}\right)^{2} \mu_{a} \\
a=\mu_{a}+\rho_{a}\left(a-\mu_{a}\right)+\sigma_{a} e^{\widehat{\sigma}_{a}} \cdot 0 \\
\widehat{\sigma}_{a}=v_{a} \widehat{\sigma}_{a}+\eta_{a} \cdot 0 \\
A \equiv e^{\widehat{a}} \text { (auxiliary equation) }
\end{gathered}
$$

Micro-Uncertainty Shock

$$
\begin{gathered}
\mu_{\omega}=-\frac{\left(\sigma_{\omega} e^{\widehat{\sigma}_{\omega}}\right)^{2}}{2} \\
\widehat{\sigma}_{\omega}=v_{\omega} \widehat{\sigma}_{\omega}+\eta_{\omega} \cdot 0 \\
O_{t} \equiv e^{\mu_{\omega}+\frac{\left(\sigma_{\omega} \hat{\sigma}^{\widehat{\sigma}}\right)^{2}}{2}} \text { (auxiliary equation) }
\end{gathered}
$$

Monetary Policy Shock (with Policy-Uncertainty)

$$
\begin{gathered}
\mu_{m}=-\frac{\left(\sigma_{m} e^{\widehat{\sigma}_{m}}\right)^{2}}{2}+\left(\rho_{m}\right)^{2} \mu_{m} \\
m=\mu_{m}+\rho_{m}\left(m-\mu_{m}\right)+\sigma_{m} e^{\widehat{\sigma}_{m}} \cdot 0 \\
\widehat{\sigma}_{m}=v_{m} \widehat{\sigma}_{m}+\eta_{m} \cdot 0 \\
M \simeq 1+\widehat{m} \text { (auxiliary equation) }
\end{gathered}
$$




\subsection{Steady State and Structural Parameters}

The zero-inflation deterministic steady state of the model can be specified as follows:

$$
\Pi=\Pi^{*}=1 .
$$

Let us impose $\widehat{a}=\widehat{m}=0$ and let's ignore the exogenous show equations in (113) - (123) for now. This ensures that the monetary policy rule in (112) is satisfied in steady state. We also assume that the characterization of the steady state implies that,

$$
\begin{aligned}
& X=\delta K, \\
& Q=1 .
\end{aligned}
$$

This satisfies (95) and (99) trivially, but it also implies from (96) that,

$$
\bar{Q}=1
$$

From (90), it follows that,

$$
P^{w r}=\frac{\epsilon-1}{\epsilon},
$$

and from (89) we get that,

$$
I=\frac{1}{\beta},
$$

while (93) simply says,

$$
H^{e}=1 \text {. }
$$

Then, we can re-write (86) in the following terms,

$$
\Lambda=(1-b \beta)(1-b)^{-\chi} C^{-\chi} .
$$

All these steady state conditions hold in equilibrium and are straightforward to characterize in setting up the deterministic steady state for the model.

The deterministic steady state of the different shock processes described in (113) - (123) 
can be summarized now as follows,

$$
\begin{aligned}
\mu_{a} & =-\frac{1}{2} \frac{\sigma_{a}^{2}}{1-\left(\rho_{a}\right)^{2}}, \\
a & =\mu_{a}, \\
\widehat{\sigma}_{a} & =0, \\
A & \equiv 1 \Leftrightarrow \widehat{a}=0,
\end{aligned}
$$

and

$$
\begin{aligned}
\mu_{\omega} & =-\frac{\sigma_{\omega}^{2}}{2}, \\
\widehat{\sigma}_{\omega} & =0, \\
O_{t} & =1,
\end{aligned}
$$

and

$$
\begin{aligned}
\mu_{m} & =-\frac{1}{2} \frac{\sigma_{m}^{2}}{1-\left(\rho_{m}\right)^{2}}, \\
m & =\mu_{m}, \\
\widehat{\sigma}_{m} & =0, \\
M & \equiv 1 \Leftrightarrow \widehat{m}=0 .
\end{aligned}
$$

Finally, the reminder of the steady state conditions not related to the loan contract-i.e., (87), (88), (91), (92), (94), (97), (98), (100) and (101) - can be re-written as,

$$
\begin{aligned}
& Y=C+\delta K+\mu G\left(\bar{\omega}, \sigma_{\omega}\right) R^{e} K, \\
& Y=e^{\widehat{a}}(K)^{\alpha}(H)^{1-\alpha-\vartheta},
\end{aligned}
$$




$$
\begin{aligned}
W^{r} & =(1-\alpha-\vartheta)\left(\frac{\epsilon-1}{\epsilon}\right) \frac{Y}{H}, \\
W^{e r} & =\vartheta\left(\frac{\epsilon-1}{\epsilon}\right) Y, \\
W^{r} & =\left(\frac{(1-b)^{\chi}}{1-b \beta}\right) \kappa H^{\xi} C^{\chi}, \\
R^{e} & =R^{w r}+(1-\delta), \\
R^{w r} & =\alpha\left(\frac{\epsilon-1}{\epsilon}\right) \frac{Y}{K}, \\
C^{e} & =(1-\gamma) \Psi\left(\bar{\omega}, \sigma_{\omega}\right) R^{e} N^{r}, \\
N^{r} & =\frac{W^{e r}}{1-\gamma \Psi\left(\bar{\omega}, \sigma_{\omega}\right) R^{e}} .
\end{aligned}
$$

The deterministic steady state is then complete with the corresponding equations that characterize the terms of the loan contract under log-normality in (102) - (111), i.e. with

$$
\begin{aligned}
\Phi\left(\bar{\omega} \mid \sigma_{\omega}\right) & =\frac{1}{2}\left[1+\operatorname{erf}\left(\frac{\ln \bar{\omega}+\frac{\sigma_{\omega}^{2}}{2}}{\sigma_{\omega} \sqrt[2]{2}}\right)\right], \\
f\left(\bar{\omega}, \sigma_{\omega}\right) & =\frac{1}{2}\left[1+\operatorname{erf}\left(\frac{\frac{\sigma_{\omega}^{2}}{2}-\ln \bar{\omega}}{\sigma_{\omega} \sqrt[2]{2}}\right)\right]-\bar{\omega}\left(1-\Phi\left(\bar{\omega} \mid \sigma_{\omega}\right)\right), \\
G\left(\bar{\omega}, \sigma_{\omega}\right) & =1-\frac{1}{2}\left[1+\operatorname{erf}\left(\frac{\frac{\sigma_{\omega}^{2}}{2}-\ln \bar{\omega}}{\sigma_{\omega} \sqrt[2]{2}}\right)\right] \\
\lambda\left(\bar{\omega}, \sigma_{\omega}\right)\left(f_{\omega}\left(\bar{\omega}, \sigma_{\omega}\right)+\mu \sigma_{\omega}\right)+g\left(\bar{\omega}, \sigma_{\omega}\right) & \left.=1-\mu G\left(\bar{\omega}, \sigma_{\omega}\right)\right) \\
f_{\omega}\left(\bar{\omega}, \sigma_{\omega}\right) & =-\frac{1}{2}\left[1+\operatorname{erf}\left(\frac{-\frac{\sigma_{\omega}^{2}}{2}-\ln \bar{\omega}}{\sigma_{\omega} \sqrt[2]{2}}\right)\right] \\
G_{\omega}\left(\bar{\omega}, \sigma_{\omega}\right) & =0.398942\left(\frac{1}{\left.\sigma_{\omega} \sqrt[2]{\bar{\omega}}\right)}\right) \\
\Psi\left(\bar{\omega}, \sigma_{\omega}\right) & =f\left(\bar{\omega}, \sigma_{\omega}\right)+\lambda\left(\bar{\omega}, \sigma_{\omega}\right) g\left(\bar{\omega}, \sigma_{\omega}\right) \\
\frac{K}{N^{r}} & =\frac{\Psi\left(\bar{\omega}, \sigma_{\omega}\right)}{f\left(\bar{\omega}, \sigma_{\omega}\right)}, \\
R^{e} & =\frac{\lambda\left(\bar{\omega}, \sigma_{\omega}\right)}{\Psi\left(\bar{\omega}, \sigma_{\omega}\right)} \frac{1}{\beta} .
\end{aligned}
$$


We solve this subset of equations to characterize the deterministic steady state of the model with frictions using the nonlinear solver fsolve in Matlab.

Remark: On Matters of Implementation. The deterministic steady state for the model can be expressed in a somewhat more tractable manner using equation (102) — which defines the probability of default in steady state $\Phi\left(\bar{\omega} \mid \sigma_{\omega}\right)$ under log-normality — as follows,

$$
\begin{aligned}
\Phi\left(\bar{\omega} \mid \sigma_{\omega}\right) & =\frac{1}{2}\left[1+\operatorname{erf}\left(\frac{\ln \bar{\omega}+\frac{\sigma_{\omega}^{2}}{2}}{\sigma_{\omega} \sqrt[2]{2}}\right)\right] \\
& \Leftrightarrow \operatorname{erf}\left(\frac{\ln \bar{\omega}+\frac{\sigma_{\omega}^{2}}{2}}{\sigma_{\omega} \sqrt[2]{2}}\right)=2 \Phi\left(\bar{\omega} \mid \sigma_{\omega}\right)-1 \\
& \Leftrightarrow \operatorname{erf}^{-1}\left(\operatorname{erf}\left(\frac{\ln \bar{\omega}+\frac{\sigma_{\omega}^{2}}{2}}{\sigma_{\omega} \sqrt[2]{2}}\right)\right)=\operatorname{erf}^{-1}\left(2 \Phi\left(\bar{\omega} \mid \sigma_{\omega}\right)-1\right) .
\end{aligned}
$$

From here, we get that,

$$
\ln \bar{\omega}=\sigma_{\omega} \sqrt[2]{2}\left[\operatorname{erf}^{-1}\left(2 \Phi\left(\bar{\omega} \mid \sigma_{\omega}\right)-1\right)\right]-\frac{\sigma_{\omega}^{2}}{2}
$$

or simply

$$
\bar{\omega}=e^{\sigma_{\omega} \sqrt[2]{2}\left[\operatorname{erf}^{-1}\left(2 \Phi\left(\bar{\omega} \mid \sigma_{\omega}\right)-1\right)\right]-\frac{\sigma_{\omega}^{2}}{2}}
$$

As a result, the steady state default threshold $\bar{\omega}$ can be expressed as a function of the steady state probability of default $\Phi\left(\bar{\omega} \mid \sigma_{\omega}\right)$. This is useful to facilitate the parameterization of the model.

\section{Data}

Through the Federal Reserve Bank of St. Louis' FRED database, we collect data on real GDP, consumption, investment and hours worked in per capita terms, together with time series for the real wage, the net-worth-to-asset ratio (or equity ratio) of nonfinancial corporations, the inflation rate, the short-term nominal interest rate and the interest rate credit risk spread for the U.S. economy. The data we use is quarterly and covers the period from 1984:Q1 to 2014:Q4.

Our measure of output is the real GDP from the U.S. Bureau of Economic Analysis' (BEA) National Income and Product Accounts (NIPA) in billions of Chained 2009 Dollars. 
Our measure of consumption is the sum of nondurable and services consumption, also in billions of Chained 2009 Dollars from the BEA's NIPA accounts. Investment is calculated as gross private fixed investment plus consumption expenditures on durable goods (since durable goods behave more similar to investment than to nondurable goods consumption). Durable consumption and investment data are also expressed in billions of Chained 2009 Dollars and reported in the BEA's NIPA accounts.

These national account series (GDP, consumption and investment) are then expressed in per capita terms after dividing them by the civilian non-institutionalized population aged 16 and over from the U.S. Bureau of Labor Statistics (BLS). This population series is simply a quarterly average of monthly data. The three of them are already reported in real terms, but we also index them to $2009=100$ and express them in logs times 400 . With these transformations we help express their cyclical component in percentages at an annualized rate after filtering each of them (consistently with what we do to the corresponding endogenous variables in the simulated model).

Total hours worked refers to the index series $(2009=100)$ on hours of all persons in the non-farm business sector reported by the BLS. This series is expressed in per capita terms divided by the civilian non-institutionalized population aged 16 and over from the BLS and re-based to $2009=100$. Then, the series is expressed in logs times 400 prior to filtering to maintain consistency with its simulated counterpart. Real wages are measured as real compensation per hour in the non-farm business sector, with the index series $(2009=100)$ obtained from the BLS. This wage series is already reported in real terms, but we also express it in logs times 400 to make it consistent with the corresponding definition in the simulated model.

The price level is measured as the implicit price deflator for GDP (2009=100), obtained from the BEA. The implicit GDP deflator is expressed in logs times 400 to keep it consistent with the corresponding model definition. We then calculate the inflation rate by simply computing the first differences of the series. The nominal short term interest rate corresponds to the three month Treasury Bill (secondary market) rate (henceforth, T-bill rate), obtained from the Board of Governors of the Federal Reserve System (H.15 Selected Interest Rates). The three-month T-bill rate is a quarterly average of daily data calculated on a discount basis, and reported annualized and in percentage terms. Therefore, the three-month T-bill rate does not have to be logged times 400 to keep it consistent with its counterpart in the model.

The interest rate credit spread is measured with the seasoned Baa corporate bond yield from Moody's relative to the yield on the 10-Year Treasury constant maturity, obtained from 
the Federal Reserve Bank of St. Louis. This spread is calculated as a quarterly average from monthly data, but does not have to be transformed in logs and multiplied by 400 to make it comparable with its endogenous counterpart from the model. The nonfinancial corporate net-worth-to-asset ratio (or equity ratio) is computed as the ratio of the net worth (level) of the nonfinancial corporate business sector over its total assets (level) times 100, using data from the Board of Governors of the Federal Reserve System (Z.1 Financial Accounts of the U.S.). This data is reported as end-of-period, at quarterly frequency. No further adjustment of the net-worth-to-asset ratio (or equity ratio) series is needed.

The Business Cycle Component and its Features. We extract the cyclical component of all these series by HP-filtering them with a one-sided filter using a lambda of 1600 and a power of 2, except for the net-worth-to-asset ratio (or equity ratio) that is demeaned instead. We apply the same filtering to the corresponding endogenous data simulated by the model to ensure the comparability between simulated and empirical moments. Some of the selected business cycle moments reported below are used for estimation with our simulated method of moment (SMM) approach and, more generally, they provide us with an empirical point of reference for the parameterization of the model.

Main files to replicate the data and examine the business cycle moments used to parameterize the model:

1. Business CyclesStylizedFacts.xlsx. This excel file contains links to the data downloaded from the Federal Reserve Bank of St. Louis' FRED database as well as the necessary calculations to compute the business cycle moments reported in the paper.

2. one_sided_hp_filter_serial.m. This matlab program extracts the cyclical component from a time series using the one-sided HP-filter based on spare matrices and exploiting their pattern. This code follows the approach described in Mehra (2004). The code was written by Alexander Meyer-Gohde.

3. one_sided_hp_filter_kalman.m. This matlab program extracts the cyclical component from a time series using the one-sided HP-filter based on implementing the Kalman filter. This code follows the approach described in page 301 of Stock and Watson (1999), as written by Alexander Meyer-Gohde. 


\section{Codes}

Driver files for simulating, estimating, and examining the benchmark model with a thirdorder approximation solution. ${ }^{13}$ These driver files also replicate the alternative model parameterizations considered in the paper.

1. BMZ_credit_moments_cases.m. This programs simulates the pruned $3^{\text {rd }}$ order approximation in order to generate stationary distribution and the model moment statistics. There are lines in the code that allows one to change specific parameter values. The results are saved to an external file (this is actually commented out in copy of the program so that existing results are not inadvertently overwritten).

2. BMZ_credit_conditional_GIRF_positive.m. This program generates conditional GIRF. To run this program one must first have generated the stationary distribution for the relevant model (see BMZ_credit_moments_cases.m). The default take the conditional at the approximately the $5^{\text {th }}$ and $95^{\text {th }}$ percentiles

3. BMZ_credit_unconditional_GIRF_positive.m. This program generates unconditional GIRF. To run this program one must first have generated the stationary distribution for the relevant model (see BMZ_credit_moments_cases.m). Here 500 starting values, randomly drawn from the unconditional distribution, are fed is an initial conditions for the IRF calculations.

4. BMZ_credit_esitmate.m. This program that estimates a select number of model parameters by SMM. The current version of the program calls initial parameter values from a file (the final estimated values). If you want to start from scratch, this would need to be commented out.

Files used for printing out and plotting results:

1. BMZ_credit_conditional_GIRF_plot.m. This graphs out various impulse response experiments. The program calls external files that contain the results of previously generated IRF experiments (both unconditional and conditional).

2. BMZ_credit_conditional_dist.m. This file graphs out various scatterplots and conditional density functions based on the unconditional distribution for a specific model. Draws from the unconditional distribution are read in from an external file.

\footnotetext{
${ }^{13}$ First- and second-order approximations do not properly account for time-varying uncertainty. See Schmitt-Grohé and Uribe (2004), Aruoba et al. (2006), Fernández-Villaverde (2010), and Martínez-García (2018), among others, for a further discussion of the relative merits of local approximation methods.
} 
3. BMZ_credit_moments_load_print.m. This file prints out a table with various moment statistics for the alternative models. The statistics are read in from various external data files.

To run these programs the "current folder" in MATLAB must be the same folder that bgg_with_SV.mod is in.

Supplementary files that are called by the driver programs and other subroutines. These files are specific for the project in this paper:

1. bgg_with_SV.mod. This is the main Dynare file for the code. This initializes Dynare and creates the global variables that will be used later to solve for the pruned $3^{\text {rd }}$ order approximation.

2. bgg_with_SV_SS.m. This matlab file is auxiliary external code needed to calculate the steady state of the model for Dynare. This program finds initial estimate of the steady state.

3. bgg_with_SV_Rev.m. This file allows the user to modify parameter values and to call modified Dynare subroutines that are then used to solve for $2^{\text {nd }}$ and $3^{\text {rd }}$ order approximations. These will be used to construct the pruned $3^{\text {rd }}$ order approximation.

4. bgg_with_SV_SS_Rev.m. This code solves for steady state (called from the bgg_with_SS_Rev.m subroutine).

The file bgg_with_SV.mod contains the compact set of equations that we use to simulate the model in our Dynare code (written for Dynare version 4.3.2 and Matlab R2012a (7.14.0.739)). Some preliminary considerations about the model and the Dynare code:

- Special cases of the model without frictions: The frictionless version of the model can be described with the same set of equilibrium conditions imposing certain restrictions on the parameterization of the code: $\varphi_{p}=0$ (no price adjustment costs), $\mu=0$ (no monitoring costs) and a policy rule that targets $\bar{\Pi}_{t}^{*}=1$ (setting the net inflation target to zero and the gross to one). This alone suffices to characterize the dynamics of the model without nominal rigidities and without the financial friction.

- Pre-determined variables and state variables in the model: There are two endogenous state variables in the model: capital, $K_{t}$, and real net worth, $N_{t}^{r} \equiv \frac{N_{t}}{P_{t}}$. We use the predetermined_variables option in Dynare to change the default convention which is 
that the timing of a variable reflects when this variable is actually decided. Hence, the endogenous variables declared as predetermined are decided one period ahead of all other endogenous variables. The convention is that $K_{t}$ is actually decided prior to $t$ with the investment decisions made at time $t-1$ and in previous periods, so it is declared as predetermined. In turn, the real net worth of entrepreneurs $N_{t}^{r}$ used to fund capital purchases at time $t$ depends on past decisions but also depends on the entrepreneurial income earned at time $t$. Given this timing, real net worth is not treated as a predetermined variable.

The following subroutines are from the "sim_folder." These subroutines are called with simulating the pruned 3rd order approximation or when generating impulse responses from the $3^{\text {rd }}$ order approximation.

1. postGIRFsim_3rd_aux.m. This subroutine generates pruned $3^{\text {rd }}$ order approximation and then calculates IRFs (given an initial condition).

2. postmoment_3rd_aux.m. This subroutine generates pruned $3^{\text {rd }}$ order approximation and the simulates and does moment calculations for the pruned $3^{\text {rd }}$ order approximation.

3. particle3rd_setup_aux.m. This subroutine constructs the components of the pruned $3^{\text {rd }}$ order approximation.

4. SIM3rd.m. Simulates the pruned $3^{\text {rd }}$ order approximation.

5. IRFSIM3rd.m. Calculates simulated estimates of the impulse responses (not currently used).

6. GIRF3rd.m. This subroutine calculates the analytical impulse responses (given an initial conditional).

7. Statemoments3rdvar.m. Calculates the unconditional mean of the model variables for pruned $3^{\text {rd }}$ order approximation.

8. stoch_simul2.m. Custom modified Dynare subroutine.

9. dynare_solve2.m. Custom modified Dynare subroutine.

10. resol2.m. Custom modified Dynare subroutine. 
11. evaluate_steady_state2.m. Custom modified Dynare subroutine.

12. k_order_pert2.m. Custom modified Dynare subroutine.

13. stochastic_solvers2.m. Custom modified Dynare subroutine.

14. mykron2.m. Custom kronecker product subroutine.

15. mykron.m. Custom kronecker product subroutine.

16. hpfilter.m. Regular 2-sided hp filter.

17. one_sided_hp_filter_kalman.m. One sided HP filter.

18. one_sided_hp_filter_serial.m. One sided HP filter.

The following files are from the GMM_folder. They are called when estimating the model by GMM/SMM.

1. SIM3rdSMM.m. This program simulates pruned $3^{\text {rd }}$ order approximation when doing SMM.

2. Estmoment.m. This subroutine calculates the empirical moment conditions used in estimation.

3. Estmoment_Wout.m. This subroutine calculates the empirical moment conditions as well as outputs the weighting matrix $W=S$.

4. GMMest_Moment_3rd.m. This subroutine generates pruned $3^{\text {rd }}$ order approximation and then calculates various moment conditions implied by the model.

5. GMMest_loss_3rd.m. Generates the empirical loss function for the GMM/SMM when using a pruned $3^{r d}$ order approximation.

6. GMMest_loss_3rd_exact.m. Generates the estimated moment conditions when using a pruned $3^{r d}$ order approximation.

7. GMMloss.m. Calculates the loss function for the moment conditions for a specific weighting matrix.

8. GMMest_Mout_3rd.m. Generates the estimated moment conditions when using a pruned $3^{\text {rd }}$ order approximation (outputs a "flag" that indicates that the model is valid). 
9. GMMest_Wout_3rd.m. Generates the estimated moment conditions and outputs the weighting matrix $W=S$ for pruned $3^{\text {rd }}$ order approximation.

10. numgrad2mod.m. Slightly modified subroutine to calculated numerical $2^{\text {nd }}$ derivatives (originally based on a program written by Chris Sims).

\section{Model Variables and Parameters}


Table 1. Correspondence Between the Notation Used in the Paper and in the Dynare Code

\begin{tabular}{|c|c|c|}
\hline Variables & Paper & Code \\
\hline \multicolumn{3}{|l|}{ Main Macro Variables } \\
\hline Output & $\ln \left(Y_{t}\right)$ & $\mathrm{y}$ \\
\hline Household Consumption & $\ln \left(C_{t}\right)$ & $\mathrm{c}$ \\
\hline Entrepreneurial Consumption & $\ln \left(C_{t}^{e}\right)$ & ce \\
\hline Capital Investment (Flow) & $\ln \left(X_{t}\right)$ & $\mathrm{x}$ \\
\hline Stock of Capital & $\ln \left(K_{t}\right)$ & $\mathrm{k}$ \\
\hline Household Labor & $\ln \left(H_{t}\right)$ & $\mathrm{h}$ \\
\hline Entrepreneurial Labor & $\ln \left(H_{t}^{e}\right)$ & he \\
\hline Real Wage for Household Labor & $\ln \left(W_{t}^{r}\right)$ & wr \\
\hline Real Wage for Entrepreneurial Labor & $\ln \left(W_{t}^{e r}\right)$ & wer \\
\hline Real Rental Rate on Capital & $\ln \left(R_{t}^{w r}\right)$ & rwr \\
\hline Real Net Worth of the Entrepreneurs & $\ln \left(N_{t}^{r}\right)$ & $\mathrm{nr}$ \\
\hline Average Nominal Return on Capital Accrued to the Entrepreneurs & $\ln \left(R_{t}^{e}\right)$ & $\operatorname{Re}$ \\
\hline Tobin's Q (Real Price of Investment) & $\ln \left(Q_{t}\right)$ & q \\
\hline Real Resale Value of Capital After Production & $\ln \left(\bar{Q}_{t}\right)$ & qbar \\
\hline Real Price of the Wholesale Good & $\ln \left(P_{t}^{w r}\right)$ & pwr \\
\hline Gross Inflation Rate & $\ln \left(\Pi_{t}\right)$ & $\mathrm{dp}$ \\
\hline Gross Nominal Interest Rate & $\ln \left(I_{t}\right)$ & i \\
\hline Lagrange Multiplier on the Household's Real Budget Constraint (Auxiliary) & $\Lambda_{t}$ & Lambdamult \\
\hline \multicolumn{3}{|l|}{ Variables Related to the Financial Contract } \\
\hline Threshold That Defines Whether Entrepreneur Defaults or Not & $\bar{\omega}_{t}$ & obar \\
\hline Fraction of Entrepreneurs that Default Every Period & $\Phi\left(\bar{\omega}_{t} \mid \mu_{\omega, t}, \sigma_{\omega, t}\right)$ & phio \\
\hline Fraction of Nominal Capital Return for Entrepreneurs & $f\left(\bar{\omega}_{t}, \sigma_{\omega, t}\right)$ & $\mathrm{f}$ \\
\hline Fraction of Nominal Capital Return for Financial Intermediaries & $g\left(\bar{\omega}_{t}, \sigma_{\omega, t}\right)$ & g \\
\hline Fraction of Nominal Capital Return Lost on Monitoring Costs $\left(\mu G\left(\bar{\omega}_{t}, \sigma_{\omega, t}\right)\right)$ & $G\left(\bar{\omega}_{t}, \sigma_{\omega, t}\right)$ & Gfun \\
\hline Lagrange Multiplier on the Lender's Participation Constraint & $\lambda\left(\bar{\omega}_{t}, \sigma_{\omega, t}\right)$ & Lambdafun \\
\hline Derivative of $f\left(\bar{\omega}_{t}, \sigma_{\omega, t}\right)$ with Respect to $\bar{\omega}_{t}$ & $f_{\omega}\left(\bar{\omega}_{t}, \sigma_{\omega, t}\right)$ & $\mathrm{fw}$ \\
\hline Derivative of $G\left(\bar{\omega}_{t}, \sigma_{\omega, t}\right)$ with Respect to $\bar{\omega}_{t}$ & $G_{\omega}\left(\bar{\omega}_{t}, \sigma_{\omega, t}\right)$ & Gfunw \\
\hline Auxiliary Variable: $\Psi\left(\bar{\omega}_{t}, \sigma_{\omega, t}\right)=f\left(\bar{\omega}_{t}, \sigma_{\omega, t}\right)+\lambda\left(\bar{\omega}_{t}, \sigma_{\omega, t}\right) g\left(\bar{\omega}_{t}, \sigma_{\omega, t}\right)$ & $\Psi\left(\bar{\omega}_{t}, \sigma_{\omega, t}\right)$ & PSIfun \\
\hline
\end{tabular}


Table 2. Correspondence Between the Notation Used in the Paper and in the Dynare Code

\begin{tabular}{|c|c|c|}
\hline Variables & Paper & Code \\
\hline \multicolumn{3}{|l|}{ Exogenous TFP and Macro-uncertainty Shocks } \\
\hline Log-TFP Shock & $a_{t} \equiv \ln \left(A_{t}\right)$ & $\mathrm{a}$ \\
\hline Time-Varying Unconditional Mean of the TFP Shock & $\mu_{a, t}$ & mua \\
\hline Time-Varying Unconditional Volatility of the TFP Shock $\left(\sigma_{a, t}=\sigma_{a} e^{\widehat{\sigma}_{a, t}}\right)$ & $\widehat{\sigma}_{a, t}$ & siga \\
\hline Shock Innovations to the Mean TFP Shock & $\varepsilon_{a, t}$ & ea \\
\hline Shock Innovations to the Volatility TFP Shock & $u_{a, t}$ & ua \\
\hline Auxiliary Variable: $A_{t} \equiv e^{a_{t}}$ & $A_{t}$ & $\mathrm{~A}$ \\
\hline \multicolumn{3}{|l|}{ Exogenous Micro-uncertainty Shock } \\
\hline Unconditional Mean of the Idiosyncratic Risk Shock & $\mu_{\omega, t}$ & muo \\
\hline Time-Varying Unconditional Volatility of the Idiosyncratic Risk Shock $\left(\sigma_{\omega, t}=\sigma_{\omega} e^{\widehat{\sigma}_{\omega, t}}\right)$ & $\widehat{\sigma}_{\omega, t}$ & sigo \\
\hline $\begin{array}{l}\text { Shock Innovations to the Volatility of the Idiosyncratic Risk Shock } \\
\qquad\left(\sigma_{\omega} \hat{\sigma}_{\omega, t}\right)^{2}\end{array}$ & $u_{\omega, t}$ & uo \\
\hline Auxiliary Variable: $O_{t} \equiv \mathbb{E}\left(\omega_{t}\right)=e^{\mu_{\omega, t}+\frac{\left(\sigma_{\omega e} e^{\omega, t}\right)}{2}}$ & $O_{t} \equiv \mathbb{E}\left(\omega_{t}\right)$ & $\mathrm{O}$ \\
\hline \multicolumn{3}{|l|}{ Exogenous Monetary Policy and Policy-uncertainty Shocks } \\
\hline Monetary Policy Shock & $m_{t}$ & $\mathrm{~m}$ \\
\hline Time-Varying Unconditional Mean of the Monetary Policy Shock & $\mu_{m, t}$ & mum \\
\hline Time-Varying Unconditional Volatility of the Monetary Policy Shock $\left(\sigma_{m, t}=\sigma_{m} e^{\widehat{\sigma}_{m, t}}\right)$ & $\widehat{\sigma}_{m, t}$ & sigi \\
\hline Shock Innovations to the Mean Monetary Policy Shock & $\varepsilon_{m, t}$ & ei \\
\hline Shock Innovations to the Volatility Monetary Policy Shock & $u_{m, t}$ & ui \\
\hline Auxiliary Variable: $M_{t} \equiv 1+m_{t}$ & $M_{t}$ & M \\
\hline
\end{tabular}


Table 3. Correspondence Between the Notation Used in the Paper and in the Dynare Code

\begin{tabular}{|c|c|c|}
\hline Variables & Paper & Code \\
\hline \multicolumn{3}{|l|}{ Auxiliary Variables for Plotting the GIRFs } \\
\hline Output (In Logs, Annualized \%) & $400 * \ln \left(Y_{t}\right)$ & $P_{-} y=400^{*} \mathrm{y}$ \\
\hline Household Consumption (In Logs, Annualized \%) & $400 * \ln \left(C_{t}\right)$ & $\mathrm{P}_{-} \mathrm{c}=400^{*} \mathrm{c}$ \\
\hline Entrepreneurial Consumption (In Logs, Annualized \%) & $400 * \ln \left(C_{t}^{e}\right)$ & $\mathrm{P}_{-} \mathrm{ce}=400^{*} \mathrm{ce}$ \\
\hline Household and Entrepreneurial Consumption (In Logs, Annualized \%) & $400 *\left(\ln \left(C_{t}\right)+\ln \left(C_{t}^{e}\right)\right)$ & $\mathrm{P}_{-}{ }^{\mathrm{c}}{ }^{\mathrm{all}}=\mathrm{P}{ }_{-} \mathrm{c}+\mathrm{P}{ }_{-} \mathrm{ce}$ \\
\hline Capital Investment (Flow) (In Logs, Annualized \%) & $400 * \ln \left(X_{t}\right)$ & $P_{-} \mathrm{x}=400^{*} \mathrm{x}$ \\
\hline Household Labor (In Logs, Annualized \%) & $400 * \ln \left(H_{t}\right)$ & $\mathrm{P}_{-} \mathrm{h}=400^{*} \mathrm{~h}$ \\
\hline Inflation Rate (Quarter-Over-Quarter Changes, Annualized \%) & $400 * \ln \left(\Pi_{t}\right)$ & $P_{-} d p=400^{*} d p$ \\
\hline Nominal One-period Risk-Free Interest Rate (Annualized \%) & $400 * \ln \left(I_{t}\right)$ & $\mathrm{P}_{-} \mathrm{i}=400 * \mathrm{i}$ \\
\hline Entrepreneurs' Net Worth Over Total Capital (\%) & $100 * \frac{N_{t}^{r}}{Q_{t} K_{t+1}}$ & $\begin{array}{c}\mathrm{P}_{-} \mathrm{lev}= \\
100^{*} \exp (\mathrm{nr}) / \exp (\mathrm{q}) * \exp (\mathrm{k}(+1))\end{array}$ \\
\hline External Finance Premium (Annualized \%) & $400 * \mathbb{E}_{t}\left(\ln \left(\frac{R_{t+1}^{e}}{I_{t}}\right)\right)$ & $\begin{array}{c}\text { P_spread }= \\
400^{*} \ln (\text { Lambdafun }(+1) / \text { PSIfun }(+1))\end{array}$ \\
\hline Fraction of Entrepreneurs that Default in Each Period & $100 * \Phi\left(\bar{\omega}_{t} \mid \mu_{\omega, t}, \sigma_{\omega, t}\right)$ & $\mathrm{P}_{-}$phio $=100^{*}$ phio \\
\hline Stock of Capital (In Logs, Annualized \%) & $400 * \ln \left(K_{t+1}\right)$ & $\mathrm{P}_{-} \mathrm{k}=400 * \mathrm{k}(+1)$ \\
\hline Real Net Worth of the Entrepreneurs (In Logs, Annualized \%) & $400 * \ln \left(N_{t}^{r}\right)$ & $\mathrm{P}_{-} \mathrm{nr}=400^{*} \mathrm{nr}$ \\
\hline Tobin's q (In Logs, Annualized \%) & $400 * \ln \left(Q_{t}\right)$ & $\mathrm{P}_{-} \mathrm{q}=400 * \mathrm{q}$ \\
\hline Real Resale Value of Capital (In Logs, Annualized \%) & $400 * \ln \left(\bar{Q}_{t}\right)$ & $\mathrm{P}_{\text {_qbar }}=400^{*}$ qbar \\
\hline Real One-period Risk-Free Interest Rate (Annualized \%) & $400 * \mathbb{E}_{t}\left(\ln \left(\frac{I_{t}}{\Pi_{t+1}}\right)\right)$ & $\mathrm{P}_{-}$realrate $=400 * \mathrm{i}-400 * \mathrm{dp}(+1)$ \\
\hline Household's Real Wages & $400 * \ln \left(W_{t}^{r}\right)$ & $\mathrm{P}_{-} \mathrm{wr}=400^{*} \mathrm{wr}$ \\
\hline Entrepreneur's Real Wages & $400 * \ln \left(W_{t}^{e r}\right)$ & $\mathrm{P}_{\text {_wer }}=400^{*}$ wer \\
\hline
\end{tabular}




\begin{tabular}{|c|c|c|c|}
\hline Preference and Technological Parameters & & code & \\
\hline Households' Intertemporal Discount Factor & $0<\beta<1$ & beta & 0.99 (Bernanke et al. (1999)) \\
\hline Households' Inverse of the Intertemporal Elasticity of Substitution & $\chi \geq 0$ & chi & 1 (Bernanke et al. (1999), Christiano et al. (2 \\
\hline Households' Inverse of the Frisch Elasticity of Labor Supply & $\xi \geq 0$ & $\mathrm{xi}$ & $\frac{1}{3}$ (Bernanke et al. (1999)) \\
\hline Households' Scaling Parameter on Labor Disutility & $\kappa \geq 0$ & kappa & 0.738 (Simulated Method of Moments) \\
\hline Households' Habit Parameter & $0 \leq b \leq 1$ & $\mathrm{~b}$ & 0.738 (Simulated Method of Moments) \\
\hline Elasticity of Substitution Across Varieties & $\epsilon>1$ & eps & $10(\operatorname{Basu}(1996))$ \\
\hline Capital Share & $0 \leq \alpha \leq 1$ & alpha & 0.35 (Bernanke et al. (1999)) \\
\hline Entrepreneurial Labor Share & $0 \leq \vartheta \leq 1$ & vartheta & 0.01 (Bernanke et al. (1999)) \\
\hline Depreciation Rate & $0<\delta \leq 1$ & delta & 0.025 (Bernanke et al. (1999)) \\
\hline \multicolumn{4}{|l|}{ Adjustment Cost $\&$ Agency Cost Parameters } \\
\hline Capital Adjustment Cost & $\varphi_{k}>0$ & phik & 3.369 (Simulated Method of Moments) \\
\hline \multirow[t]{2}{*}{ Rotemberg (1982) Price Adjustment Cost } & $\varphi_{p} \geq 0$ & phip & 121.73 (Simulated Method of Moments) \\
\hline & & phipstar & 0 (without price stickiness) \\
\hline \multirow[t]{2}{*}{ Monitoring Cost } & $0 \leq \mu<1$ & $\mathrm{mu}$ & 0.145 (Simulated Method of Moments) \\
\hline & & & 0 (without monitoring costs) \\
\hline Survival Rate of Entrepreneurs & $0<\gamma<1$ & gamma & 0.978 (Simulated Method of Moments) \\
\hline \multicolumn{4}{|l|}{ Taylor Rule Policy Parameters } \\
\hline Interest Rate Smoothing & $0 \leq \rho_{i}<1$ & rhoi & 0.836 (Born and Pfeifer (2014)) \\
\hline Sensitivity to Inflation Deviations from Target & $\psi_{\pi}>1$ & psidp & 1.777 (Born and Pfeifer (2014)) \\
\hline Sensitivity to Output Gap & $\psi_{x}>0$ & psix & 0.319 (Born and Pfeifer (2014)) \\
\hline \multicolumn{4}{|l|}{ Exogenous Shock Parameters } \\
\hline Unconditional Std. Dev. of Idiosyncratic Risk Shock & $\sigma_{\omega}>0$ & stdevo & 0.300 (Simulated Method of Moments) \\
\hline Persistence of the Stochastic Volatility of Idiosyncratic Risk Shock & $0<v_{\omega}<1$ & rhosigo & 0.966 (Simulated Method of Moments) \\
\hline Std. Dev. of the Stochastic Volatility of Idiosyncratic Risk Shock & $\eta_{\omega} \geq 0$ & stdevsigo & 0.0254 (Simulated Method of Moments) \\
\hline TFP Shock Persistence & $0<\rho_{a}<1$ & rhoa & 0.814 (Born and Pfeifer (2014)) \\
\hline TFP Shock Unconditional Standard Deviation & $\sigma_{a}>0$ & stdeva & $e^{-5.233}=0.0054($ Born and Pfeifer (2014)) \\
\hline Persistence of the Stochastic Volatility on TFP & $0<v_{a}<1$ & rhosiga & 0.632 (Born and Pfeifer (2014)) \\
\hline Std. Dev. of the Stochastic Volatility on TFP & $\eta_{a} \geq 0$ & stdevsiga & 0.312 (Born and Pfeifer (2014)) \\
\hline Monetary Shock Persistence & $0<\rho_{m}<1$ & rhom & 0.367 (Born and Pfeifer (2014)) \\
\hline Monetary Shock Unconditional Standard Deviation & $\sigma_{m}>0$ & stdevi & $e^{-6.551}=0.0014($ Born and Pfeifer $(2014))$ \\
\hline Persistence of the Stochastic Volatility of Monetary Shock & $0<v_{m}<1$ & rhosigi & 0.921 (Born and Pfeifer (2014)) \\
\hline Std. Dev. of the Stochastic Volatility of Monetary Shock & $\eta_{m} \geq 0$ & stdevsigi & 0.363 (Born and Pfeifer (2014)) \\
\hline
\end{tabular}




\section{Quantitative Findings}

1. Figure 1. We explore the responses of output, the credit spread, and the nominal interest rate to a +1 shock, conditional on high uncertainty (micro-uncertainty, TFP uncertainty and monetary policy uncertainty, individually), Figure 1A captures the non-normalized responses while Figure 1B describes the response normalized by the size of each shock innovation. We show here that TFP uncertainty and monetary policy uncertainty effects are largely driven by the size of their volatility. Figure $1 \mathrm{C}$ shows the normalized responses for the case where we eliminate the monitoring costs $(\mu=0)$ which shuts down the financial accelerator channel. This shows that the propagation of shocks through the credit spread is no longer present-only microuncertainty matters to explain movements of the credit spread. Finally, Figure 1D represents the generalized impulse responses of output to all shocks conditional on the values of macro- and policy-uncertainty showcasing the scalability of TFP shocks and monetary policy shocks (both level shocks) to their own uncertainty.

2. Figure 2. We explore the responses of output, the credit spread, the nominal interest rate and other key macro-finance variables (inflation, Tobin's Q, investment, the default probability, aggregate net-worth-to-asset ratio or equity ratio, and real net worth) to $\mathrm{a}+1 /-1$ standard deviation shock conditional on high, mid, and low values of the endogenous credit spread. Figure 2A plots those responses to a TFP shock, Figure 2B to a TFP stochastic volatility shock, Figure $2 \mathrm{C}$ to a micro-uncertainty shock, Figure 2D to an interest rate rule shock, and Figure $2 \mathrm{E}$ to an interest rate rule stochastic volatility shock. The evidence shows that the propagation of one standard deviation shocks is largely symmetric. We also note that micro-uncertainty shocks tend to have much larger effects than other stochastic volatility shocks (TFP uncertainty and monetary policy uncertainty) - yet monetary policy uncertainty can have stronger real effects than TFP uncertainty. ${ }^{14}$

3. Figure 3. We explore the responses of output, the credit spread, the nominal interest rate and other key macro-finance variables (inflation, Tobin's Q, investment, the default probability, aggregate net-worth-to-asset ratio or equity ratio, and real net worth) to

\footnotetext{
${ }^{14}$ Credit spreads introduce an important nonlinearity that arises from the credit friction with which we have augmented the standard medium-scale New Keynesian setup. Still, as the time-varying stochastic volatility on TFP and monetary policy shocks shows up in the higher order ( $3^{\text {rd }}$ and above) approximation terms, the lack of substantial nonlinearities in the neighborhood of the deterministic steady state other than that embedded in the credit spread means these higher-order terms are relatively small. Hence, the direct effects of macro- and policy-uncertainty shocks remain relatively small with and without credit frictions.
} 
a $+2 /-2$ standard deviation shock conditional on high, mid, and low values of the endogenous credit spread. Figure 3A plots those responses to a TFP shock, Figure 3B to a TFP stochastic volatility shock, Figure 3C to a micro-uncertainty shock, Figure 3D to an interest rate rule shock, and Figure $3 \mathrm{E}$ to an interest rate rule stochastic volatility shock. The evidence complements that of Figure 2 showing that shocks that are twice as large (which occur less frequently too) have more sizeable effects, but generally those effects are approximately twice bigger. Therefore, findings do not appear to show that larger (and less frequent) shocks will have an effect above and beyond what one would expect given the increase in the size of the shock.

4. Figure 4. We explore the responses of output, the credit spread, the nominal interest rate and other key macro-finance variables (inflation, Tobin's Q, investment, the default probability, aggregate net-worth-to-asset ratio or equity ratio, and real net worth) to a $+1 /-1$ standard deviation shock conditional on high, mid, and low values of the micro-uncertainty shock. Figure 4A plots those responses to a TFP shock, Figure 4B to a TFP stochastic volatility shock, Figure 4C to a micro-uncertainty shock, Figure 4D to an interest rate rule shock, and Figure $4 \mathrm{E}$ to an interest rate rule stochastic volatility shock. The evidence shows that the propagation of one standard deviation shocks is largely symmetric. We also note that micro-uncertainty shocks tend to have much larger effects than other stochastic volatility shocks (TFP and the monetary policy rule) - yet monetary policy uncertainty can have stronger real effects than TFP uncertainty. Finally, we find that the results conditional on the level of micro-uncertainty are very similar to those reported based on the level of the endogenous credit spread (Figure 2).

5. Figure 5. We illustrate in Figure 5A the responses of output, the credit spread, the nominal interest rate and other key macro-finance variables (investment, Tobin's Q, inflation, consumption, hours worked, and net-worth-to-asset ratio or equity ratio) to $\mathrm{a}+1 /-1$ standard deviation shock (TFP shock, TFP uncertainty, micro-uncertainty, monetary policy shock, monetary policy uncertainty) whenever the initial conditions are set equal to the unconditional mean. We illustrate in Figure $5 \mathrm{~B}$ and Figure $5 \mathrm{C}$ the responses of output, the credit spread, the nominal interest rate to $\mathrm{a}+1 /-1$ standard deviation shock (TFP shock, TFP uncertainty, micro-uncertainty, monetary policy shock, monetary policy uncertainty) whenever we consider setting the initial conditions conditional on the $5^{\text {th }}, 50^{\text {th }}$, and $95^{\text {th }}$ percentiles of the endogenous credit spread (Figure 5B) and whenever we consider setting the initial conditions conditional 
on the $1^{s t}, 50^{t h}$, and $99^{t h}$ percentiles of the endogenous credit spread (Figure 5C). Our results show that the initial conditions have a modest effect on the propagation of the shocks.

6. Figure 6. We show in Figure $6 \mathrm{~A}$ the responses of output, the credit spread, and the nominal interest rate to a +1 standard deviation of the shocks (TFP shock, TFP uncertainty, micro-uncertainty, monetary policy shock, monetary policy uncertainty) conditional on high credit spreads, under alternative specifications: the baseline compared against a model with high relative risk aversion or low intertemporal elasticity of substitution $(\chi=7)$, with no monitoring costs and no financial accelerator $(\mu=0)$, and with no nominal rigidities $\left(\varphi_{p}=0\right)$. There findings indicate that the monitoring costs and the risk aversion parameter play a significant role in the propagation of shocks and their effectiveness through the financial accelerator channel (nominal rates and credit spreads). In turn, nominal rigidities appear particularly important for the propagation of monetary policy and even monetary policy uncertainty shocks. Figure 6B conducts the same exercise plotting the responses of output, the credit spread, and the nominal interest rate to $\mathrm{a}+1$ standard deviation of the shocks (TFP shock, TFP uncertainty, micro-uncertainty, monetary policy shock, monetary policy uncertainty) conditional on high and low credit spreads, under the baseline specification and an alternative specification without micro-uncertainty stochastic volatility. This exercise comes to show that the addition of micro-uncertainty shocks adds another source of fluctuations to the model, but has otherwise only modest effects on the propagation path for the rest of the shocks (except perhaps on the effects it has on the credit spread). We interpret this as showing that augmenting the model with micro-uncertainty does not alter all that much what we know about the propagation of other shocks. Figure $6 \mathrm{C}$ considers another experiment where we report the responses of output, the credit spread, and the nominal interest rate to a +1 standard deviation of the shocks (TFP shock, TFP uncertainty, micro-uncertainty, monetary policy shock, monetary policy uncertainty) conditional on high credit spreads, and high uncertainty (micro-uncertainty, TFP uncertainty, and monetary policy uncertainty) individually. Our findings most notably reveal that periods of high macro (TFP) uncertainty lead to significantly larger effects of TFP level shocks on real economic activity than in any other circumstance. Similarly, periods of high monetary policy uncertainty lead to significantly larger effects of the monetary policy shock on real economic activity (almost twice as large on impact) than in any other circumstance. Figure 6D provides another illustrative experiment 
where the responses of output, the credit spread, and the nominal interest rate to a +1 standard deviation of the shocks (TFP shock, TFP uncertainty, micro-uncertainty, monetary policy shock, monetary policy uncertainty) conditional on all three stochastic volatility shocks (micro-uncertainty, TFP, and monetary policy) being either high $\left(>80^{t h}\right.$ percentile) or low $\left(<20^{\text {th }}\right.$ percentile) simultaneously. Our findings show that periods of high uncertainty across all stochastic volatility shocks and low uncertainty have only modest effects on the propagation of uncertainty shocks themselves. However, our evidence suggests that the response of real economic activity to TFP level shocks is markedly stronger in periods of high overall uncertainty and, similarly, that the response to monetary policy shocks can be more than twice as large during periods of high overall uncertainty.

7. Figure 7. We show in Figure 7 the responses of output, the credit spread, the nominal interest rate, and other macro-finance variables (investment, Tobin's Q, inflation, consumption, hours worked, and net-worth-to-asset ratio or equity ratio) to a +1 standard deviation of each one of the shocks (TFP shock, TFP uncertainty, micro-uncertainty, monetary policy shock, monetary policy uncertainty) conditional on mid-values of the endogenous credit spreads, under alternative specifications: the baseline compared against a model with high relative risk aversion or low intertemporal elasticity of substitution $(\chi=7)$, with no monitoring costs and no financial accelerator $(\mu=0)$, and with no nominal rigidities $\left(\varphi_{p}=0\right)$. Our findings show that reducing nominal rigidities tends to mitigate the real effects of TFP shocks while high risk-aversion amplifies those effects for mid-values of the endogenous credit spread (Figure 7A), while the responses to TFP uncertainty are rather modest across model specifications (Figure 7B). We also show that micro-uncertainty and its propagation depends crucially on the presence of a financial accelerator channel (Figure 7C) - hence, the interaction between credit frictions and micro-uncertainty is a crucial aspect of our model. Not surprisingly, the propagation of monetary policy shocks depends critically on the presence of nominal rigidities in the model (Figure 7D) and similarly for the propagation of monetary policy uncertainty (Figure 7E). The propagation of monetary policy uncertainty and its real effects appear to become amplified whenever the risk aversion is high-interestingly, the monetary policy uncertainty shock appears to have some effect even when there are no nominal rigidities mainly because it still influences the endogenous credit spreads implied by the nominal financial contract.

8. Figure 8. We show in Figure 8 the responses of output, the credit spread, the nominal 
interest rate, and other macro-finance variables (investment, Tobin's Q, inflation, consumption, hours worked, and net-worth-to-asset ratio or equity ratio) to a +1 standard deviation of each one of the shocks (TFP shock, TFP uncertainty, micro-uncertainty, monetary policy shock, monetary policy uncertainty) conditional on high-values of the endogenous credit spreads, under alternative specifications: the baseline compared against a model with high relative risk aversion or low intertemporal elasticity of substitution $(\chi=7)$, with no monitoring costs and no financial accelerator $(\mu=0)$, and with no nominal rigidities $\left(\varphi_{p}=0\right)$. Our findings show that reducing nominal rigidities tends to mitigate the real effects of TFP shocks while high risk-aversion amplifies those effects for mid-values of the endogenous credit spread (Figure 8A), while the responses to TFP uncertainty are rather modest across model specifications (Figure 8B). We also show that micro-uncertainty and its propagation depends crucially on the presence of a financial accelerator channel (Figure 8C)-hence, the interaction between credit frictions and micro-uncertainty is a crucial aspect of our model. Not surprisingly, the propagation of monetary policy shocks depends critically on the presence of nominal rigidities in the model (Figure 8D) and similarly for the propagation of monetary policy uncertainty (Figure 7E). Qualitatively these results are the same as those reported in Figure 7 conditional on mid-values of the endogenous credit spread, except that we observe high credit spreads tend to attenuate the effect of TFP level shocks and amplify somewhat the effect of the monetary policy shock itself. In turn, the effect of high credit spreads on the propagation of all forms of uncertainty is rather modest.

9. Figure 9. We show in Figure 9 the responses of output, the credit spread, the nominal interest rate, and other macro-finance variables (investment, Tobin's Q, inflation, consumption, hours worked, and net-worth-to-asset ratio or equity ratio) to a +1 standard deviation of each one of the shocks (TFP shock, TFP uncertainty, micro-uncertainty, monetary policy shock, monetary policy uncertainty) conditional on high-values of the endogenous credit spread, high-values of the nominal interest rate, and high-values of the net-worth-to-asset ratio (or equity ratio). Figure 9A suggests that the response of the credit spread to TFP level shocks might be somewhat muted with high interest rates, while Figure 9B indicates that there are only small differences in the transmission of TFP uncertainty shocks. Figure $9 \mathrm{C}$ shows that the propagation of micro-uncertainty shocks is somewhat more muted with high net-worth-to-asset ratio (or equity ratio) and particularly with high interest rates. Figure 9D suggests that monetary policy shocks are amplified with high interest rates and dampened somewhat with high net- 
worth-to-asset ratio (or equity ratio), while Figure $9 \mathrm{E}$ indicates that the differences in the transmission of monetary policy uncertainty are rather modest.

10. Figure 10. We show in Figure 10 the responses of output, the credit spread, the nominal interest rate, and other macro-finance variables (investment, Tobin's Q, inflation, consumption, hours worked, and net-worth-to-asset ratio or equity ratio) to $\mathrm{a}+1$ standard deviation of each one of the shocks (TFP shock, TFP uncertainty, micro-uncertainty, monetary policy shock, monetary policy uncertainty) conditional on high-values of the endogenous credit spread, high-values of TFP uncertainty, highvalues of interest rate uncertainty, and high-values of the micro-uncertainty. Figure 10A suggests that the real effects of a TFP level shock might be amplified with high TFP uncertainty, while Figure 10B indicates that there are only small differences in the transmission of TFP uncertainty shocks. Figure 10C shows that the propagation of micro-uncertainty shocks through the endogenous credit spread is somewhat more muted with high interest rate uncertainty and high TFP uncertainty. Figure 10D suggests that monetary policy shocks are significantly amplified with high interest rate uncertainty, while Figure 10E indicates that the differences in the transmission of monetary policy uncertainty are rather modest with small amplification effects arising from high interest rate uncertainty.

11. Figure 11. We show in Figure 11 the probability density of the volatility of TFP, the TFP level, the micro-uncertainty volatility, the net-worth-to-asset ratio or equity ratio, the monetary policy rule volatility, and the interest rate, all of them conditional on the endogenous credit spread: unconditional, conditional on $5^{\text {th }}$ percentile of the endogenous credit spread, and conditional on the $95^{t h}$ percentile of the credit spread. The density is smoothed by the Epanechnikov kernel. Our findings illustrate the differences between the different percentiles of the conditioning variable (the credit spread) - most notably, we show that the distribution of the micro-uncertainty volatility is shifted to the left and that of the nominal interest rate is shifted to the right whenever the endogenous credit spread is conditioned at the $5^{\text {th }}$ percentile.

12. Figure 12. We show in Figure 12 the joint distribution of the endogenous credit spread with a number of relevant macro-finance variables. Figure 12A illustrates the joint distribution between the endogenous credit spread and TFP, Figure 12B the joint distribution between the endogenous credit spread and the nominal interest rate, Figure $12 \mathrm{C}$ the joint distribution of the endogenous credit spread and TFP stochastic volatility 
(TFP uncertainty), Figure 12D the joint distribution of the endogenous credit spread and the interest rate stochastic volatility (monetary policy uncertainty), Figure 12E the joint distribution of the endogenous credit spread and micro-uncertainty, Figure $12 \mathrm{~F}$ the joint distribution of the endogenous credit spread and capital, Figure $12 \mathrm{G}$ the joint distribution of the endogenous credit spread and real net worth, and Figure $12 \mathrm{H}$ the joint distribution of the endogenous credit spread and output. We observe that there is a noticeable nonlinearity in the relationship between the endogenous credit spread and the nominal interest rate as well as in the relationship between the endogenous credit spread and micro-uncertainty. Moreover, we also find nonlinearities in the relationship between the endogenous credit spread and both capital and output.

13. Figure 13. We show in Figure 13 the joint distribution of the endogenous credit spread with a number of relevant macro-finance variables whenever the households' inverse of the intertemporal elasticity of substitution is increased to $\chi=7$. Figure 13A illustrates the joint distribution between the endogenous credit spread and TFP, Figure 13B the joint distribution between the endogenous credit spread and the nominal interest rate, Figure 13C the joint distribution of the endogenous credit spread and TFP stochastic volatility (TFP uncertainty), Figure 13D the joint distribution of the endogenous credit spread and the interest rate stochastic volatility (monetary policy uncertainty), Figure 13E the joint distribution of the endogenous credit spread and micro-uncertainty, Figure $13 \mathrm{~F}$ the joint distribution of the endogenous credit spread and capital, Figure $13 \mathrm{G}$ the joint distribution of the endogenous credit spread and real net worth, and Figure 13H the joint distribution of the endogenous credit spread and output. We note that the increase in the parameter $\chi$ (which also determines the risk aversion) produces similar results to those reported under the baseline in Figure 12. It's worth noticing that higher values of $\chi$ lead to a higher frequency of zero-lower bound occurrences whereby the interest rate may fall near or below zero. Moreover, we also see that low interest rate episodes tend to be more strongly associated with periods of high endogenous credit spreads.

\section{On the Direct Effects of Uncertainty: Second Moment Shocks, First-Order Ef-} fects? We obtain relatively small effects from fluctuations in aggregate uncertainty (modeled as stochastic volatility in TFP and monetary policy). Aside from implying that macro shocks are on average larger in magnitude when aggregate uncertainty is high, this type of uncertainty is of second-order importance in the baseline model. Increasing risk aversion 
increases the effects of these uncertainty shocks, but they are still relatively small compared to shocks that affect the level of TFP or monetary policy directly.

Why are the macro- and policy-uncertainty effects relatively small?

It is because the primary effect of aggregate uncertainty in our model appears to work through the precautionary saving motive which is generally modest. Even though we have adjustment costs for changing the prices and the capital stock, the option value of waiting when making decisions that are costly to undo still doesn't appear to have large quantitative effects. Another way to think about why uncertainty has relatively small effects is there is simply not a lot of curvature in the model. The standard neoclassical medium-size DSGE model - and even the New Keynesian variant that we consider here - is not that far from being log-linear, and the addition of quadratic adjustment costs for price changes as well as adjustment costs on capital (real rigidities that introduce a real options value of waiting motive) does not add much more nonlinear structure. As the time-varying stochastic volatility shows up in the higher order ( $3^{r d}$ and above) approximation terms, the lack of substantial nonlinearities in the neighborhood of the deterministic steady state means these higher-order terms are relatively small. Perhaps, additional adjustment costs such as the costs of changing labor input might provide greater avenues for uncertainty to matter. Alternatively, adding additional sources of uncertainty might increase the quantitative influence of aggregate uncertainty. However, given the results of Born and Pfeifer (2014), other forms of uncertainty (such as on taxes) are not likely to have large effects either.

In contrast, we find that micro-uncertainty is important both qualitatively and quantitatively in our analysis - we would say of first-order importance. It is not the micro-uncertainty in and of itself that matters, but the fact that this form of uncertainty exacerbates the credit frictions that arise because of asymmetric information. In our benchmark model, taking away the credit frictions kills the financial accelerator channel and removes any role that micro-uncertainty can play on economic activity. If there are other frictions that are related to micro-uncertainty (e.g., firm hiring decisions or firm-specific adjustment costs), then increases in micro-uncertainty could have an additional effect independent of credit frictions.

While micro-uncertainty and credit frictions interact with one another, the interactions between aggregate uncertainty (on TFP or on monetary policy) and credit frictions are relatively small. The effects of aggregate uncertainty shocks do not depend on the current credit conditions, nor does the effect of a credit friction shock (micro-uncertainty) depend significantly on current aggregate uncertainty (neither related to TFP nor to monetary policy). In fact, credit spread fluctuations appear to be driven primarily by exogenous shocks in micro-uncertainty. All the macro (TFP and monetary policy) shocks including to the level 
and to uncertainty have relatively small effects on the credit spread.

What accounts for the minimal interaction between credit frictions and the macro shocks?

The only way for macroeconomic conditions to affect the credit spread is through their effect on the net-worth-to-asset ratio $\left(100 \times \frac{N_{t}}{P_{t} Q_{t} K_{t+1}}\right)$. It turns out that while macro shocks can have a large effect on individual components of the net-worth-to-asset ratio ratio, these affect the numerator and denominator of the net-worth-to-asset ratio ratio roughly in the same proportion (through their effect on the price of capital or Tobin's q). As a result, the net-worth-to-asset ratio moves very little which in turn implies a small response by the credit spread. Consequently, only changes in the exogenous micro-uncertainty have substantial effects on the credit spread and on the extent of credit frictions.

The inverse relationship between the endogenous external finance premium and the nominal interest rate found in Balke et al. (2017) suggests that low nominal short-term interest rates tend to be associated with periods of high spreads (due to high financial risk or micro-uncertainty). Hence, our research shows that periods of high financial risk (or microuncertainty) like those experienced during the Great Recession of 2008 - 2009 tend to be associated with high endogenous credit spreads and low interest rates. The message is clear: financial risks can be a major drag on economic activity while they also partly contribute to account for the low interest rate environment that has characterized the aftermath of the $2008-2009$ recession. 
Figure 1A. Generalized Impulse Response Functions: Response to a +1 Shock, Conditional on High Uncertainty, Individually (Non-Normalized).
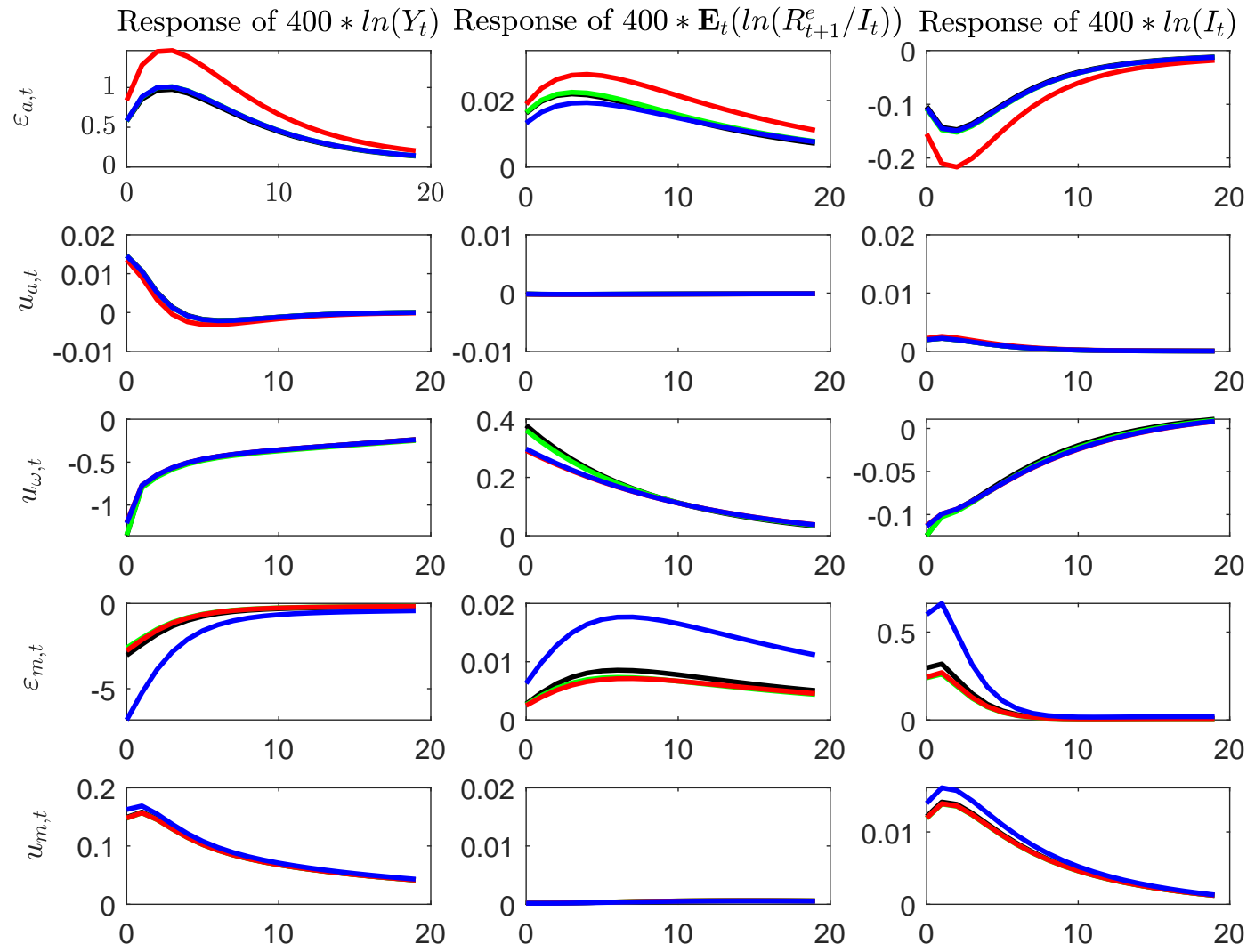

High spread - High micro uncertainty

High TFP uncertainty

High monetary uncertainty 
Figure 1B. Generalized Impulse Response Functions: Response to a +1 Shock, Conditional on High Uncertainty, Individually (Normalized by Initial Size of the Underlying Shock).
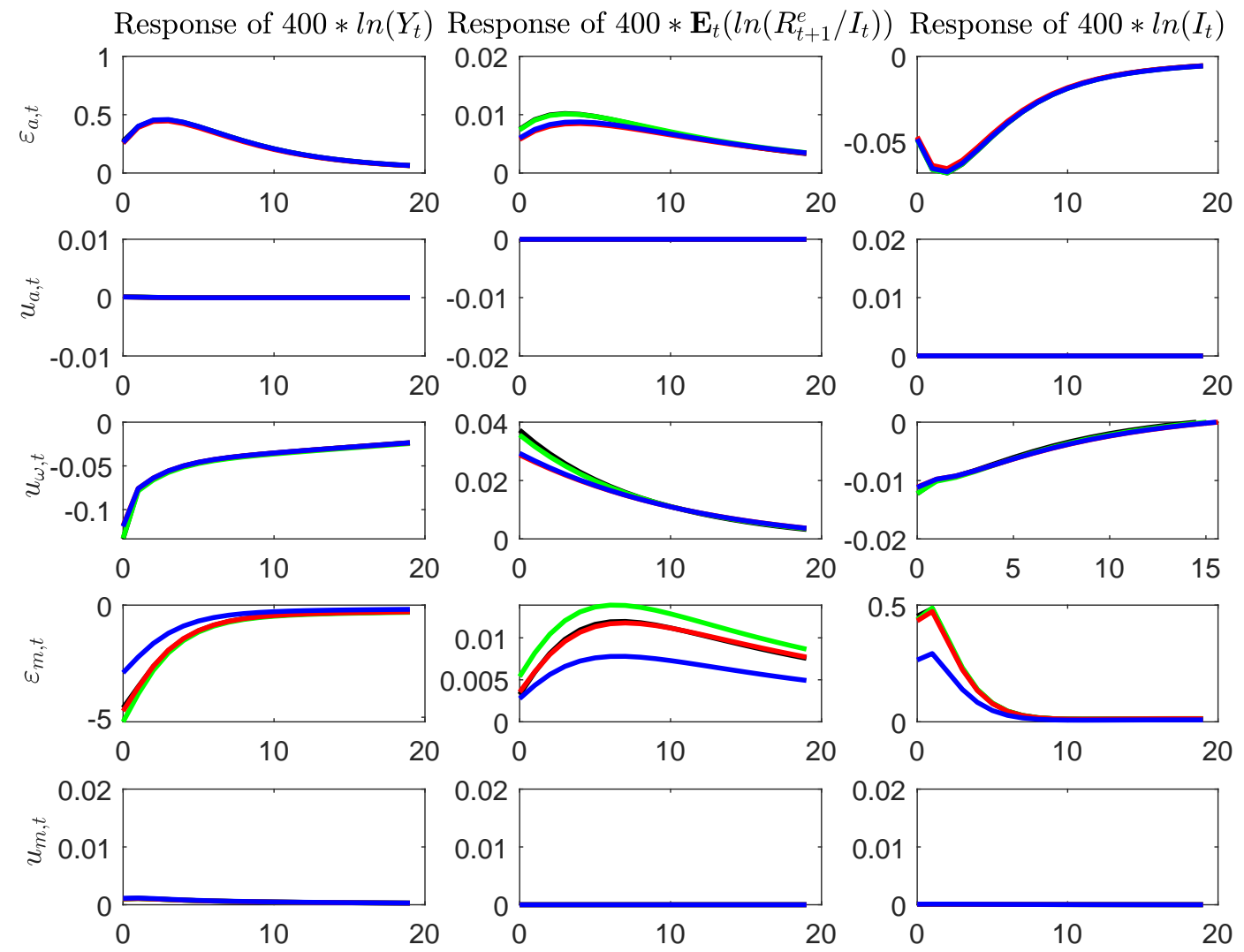

High spread $=$ High micro uncertainty — High TFP uncertainty

- High monetary uncertainty 
Figure 1C. Generalized Impulse Response Functions: Response to a +1 Shock, Conditional on High Uncertainty, Individually (Normalized by Initial Size of the Underlying Shock) whenever Default Costs Are Set to Zero $(\mu=0)$ in Order to Abstract from the Financial Accelerator Mechanism in the Model.
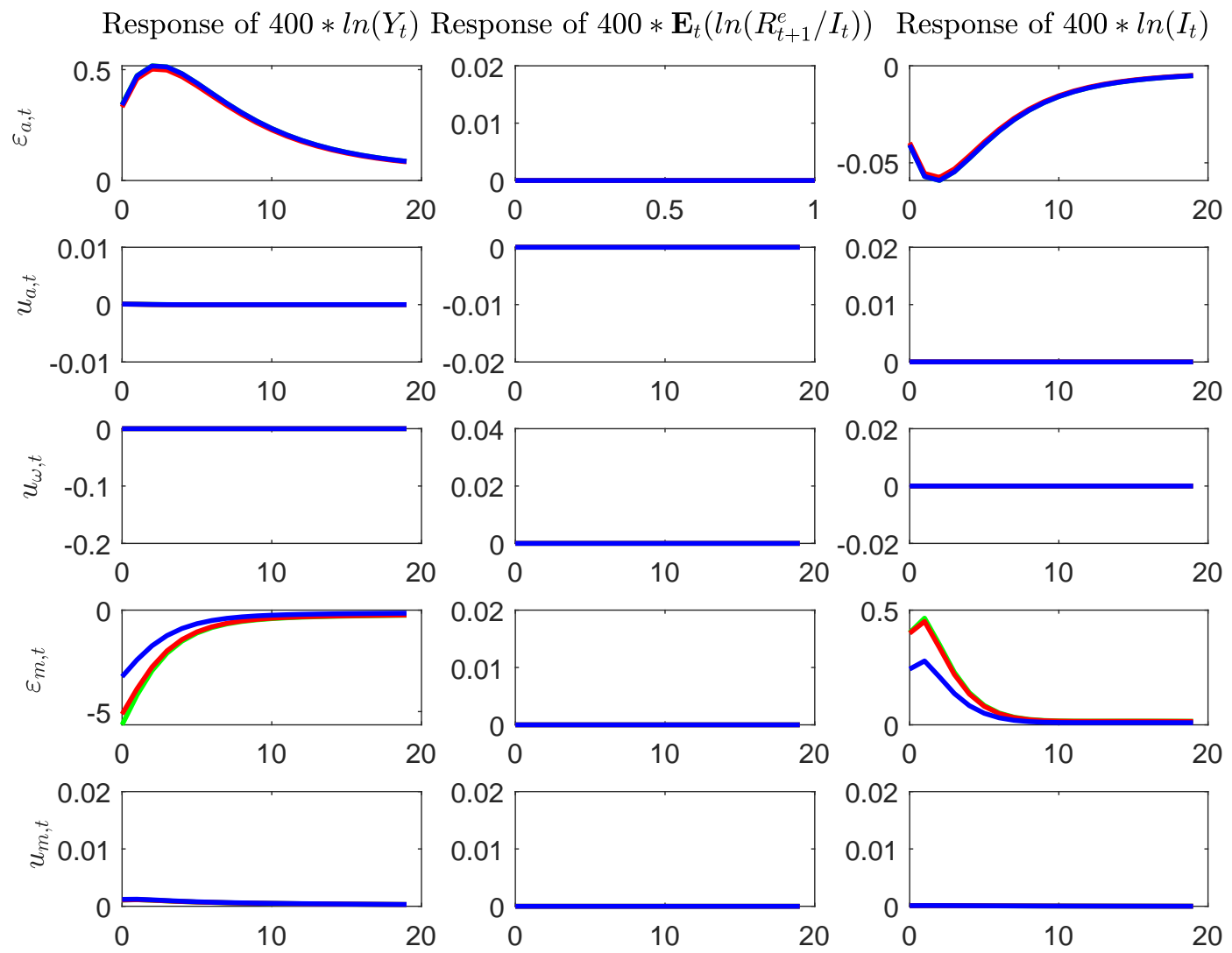

High micro uncertainty — High TFP uncertainty — High monetary uncertainty 


\section{Figure 1D. Generalized Impulse Response Functions: Response of Output to a +1 Shock, Conditional on the Values (High and Low) of Macro- and Policy-Uncertainty, Individually (Non-Normalized).}
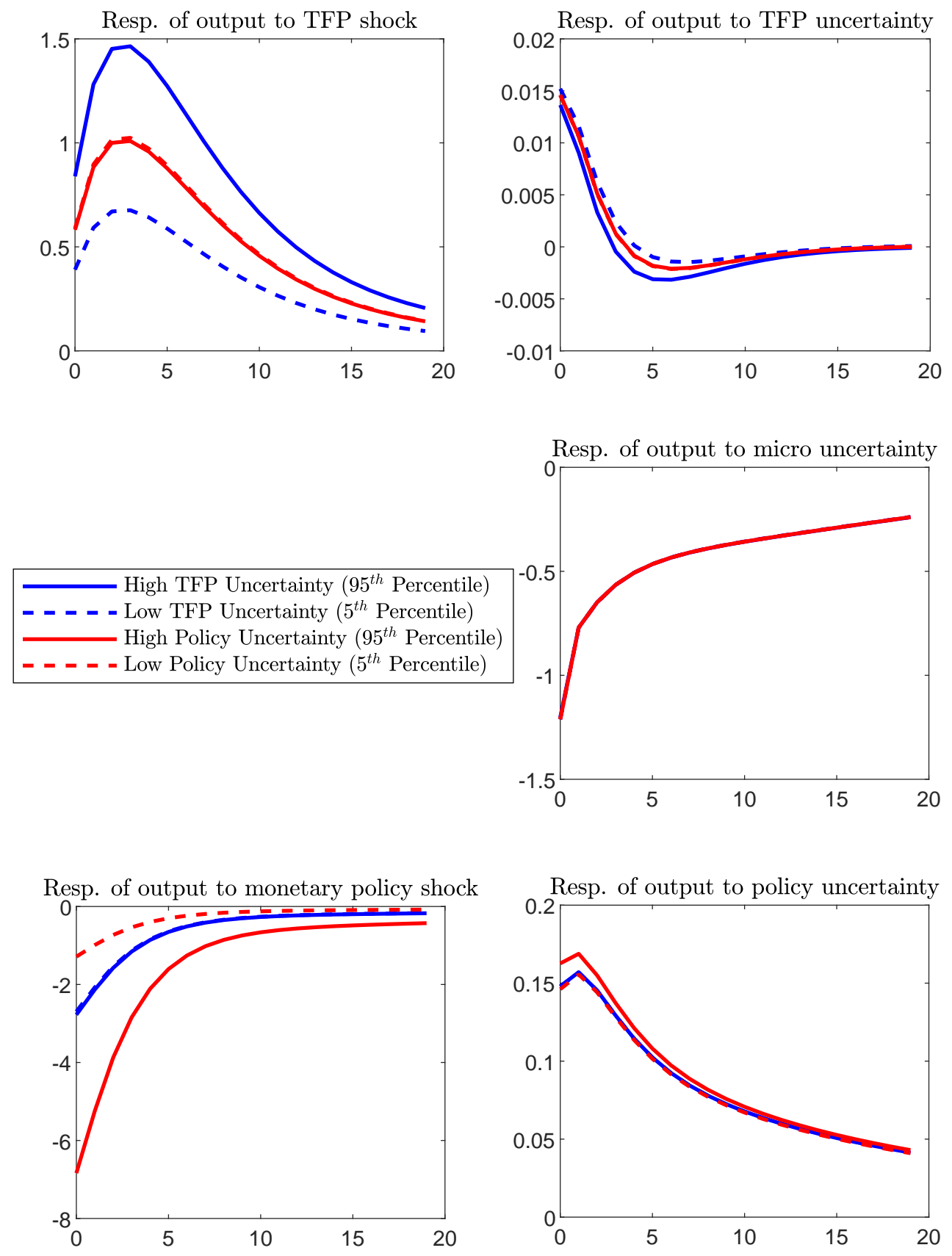
Figure 2A. Generalized Impulse Response Functions: Response to a $+\mathbf{1} /-\mathbf{1}$ Standard Deviation TFP Shock, Conditional on High, Mid, Low Values of the Endogenous Spread.
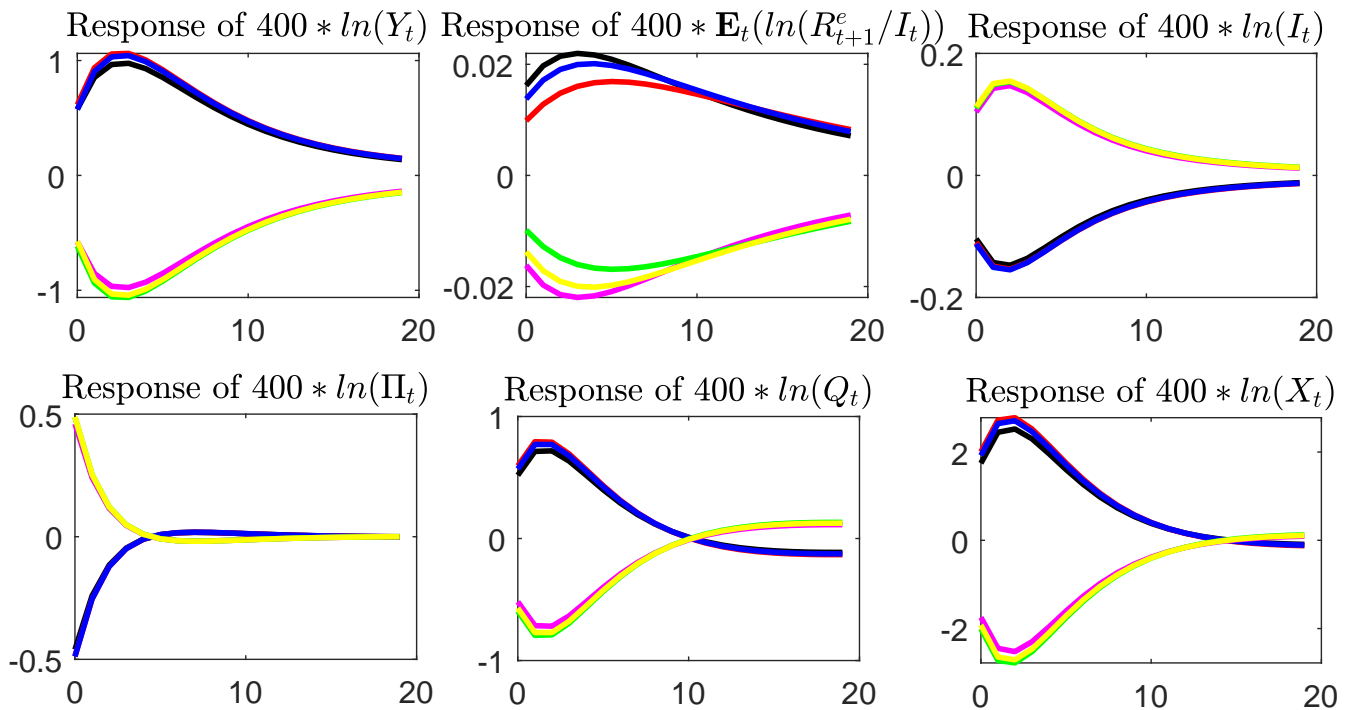

Response of $400 * \ln \left(X_{t}\right)$

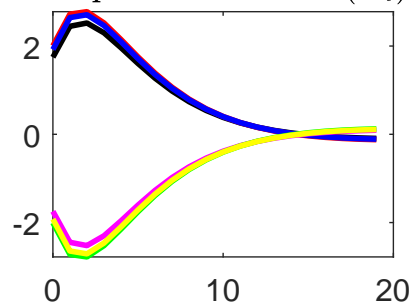

Response of $100 * \Phi\left(\bar{\omega}_{t} \mid \mu_{\omega, t}, \sigma_{\omega, t}\right)$ Response of $100 * N_{t}^{r} / Q_{t} K_{t+1}$
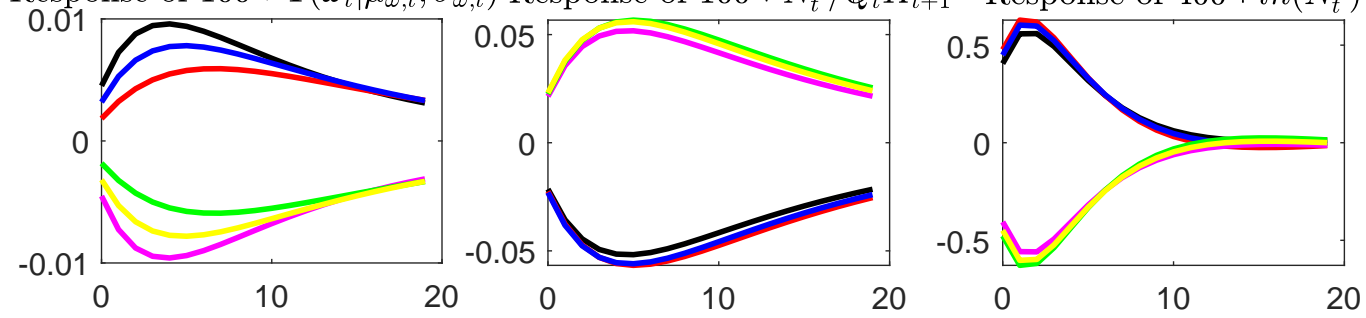

$+1 \mathrm{sd}-$ high

$+1 \mathrm{sd}-$ low $\longrightarrow+1 \mathrm{sd}-$ medium

$-1 \mathrm{sd}-$ high

$-1 \mathrm{sd}-\mathrm{low}$

$-1 \mathrm{sd}-$ medium 
Figure 2B. Generalized Impulse Response Functions: Response to a +1/-1 Standard Deviation TFP Stochastic Volatility Shock, Conditional on High, Mid, Low Values of the Endogenous Spread.
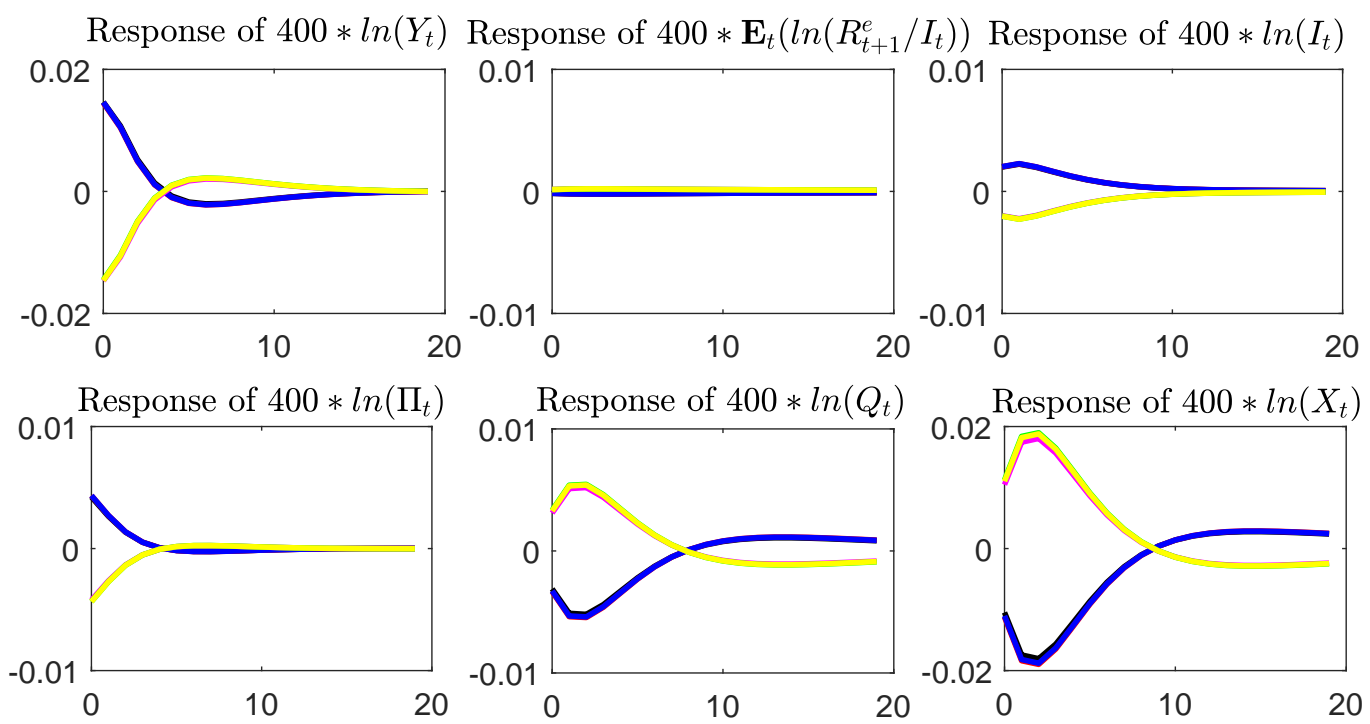

Response of $100 * \Phi\left(\bar{\omega}_{t} \mid \mu_{\omega, t}, \sigma_{\omega, t}\right)$ Response of $100 * N_{t}^{r} / Q_{t} K_{t+1} \quad$ Response of $400 * \ln \left(N_{t}^{r}\right)$
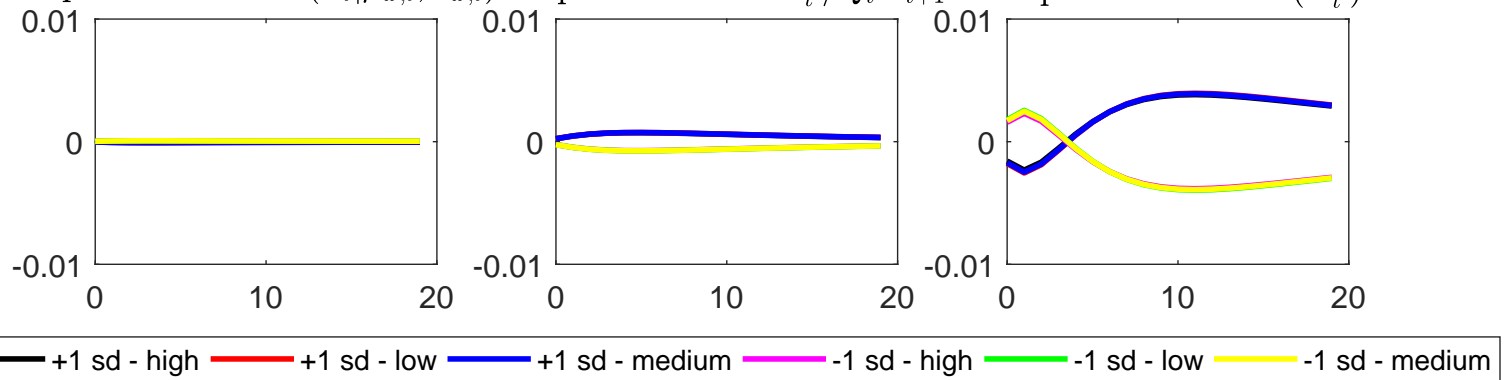
Figure 2C. Generalized Impulse Response Functions: Response to a $+\mathbf{1} / \mathbf{- 1}$ Standard Deviation Micro-Uncertainty Shock, Conditional on High, Mid, Low Values of the Endogenous Spread.
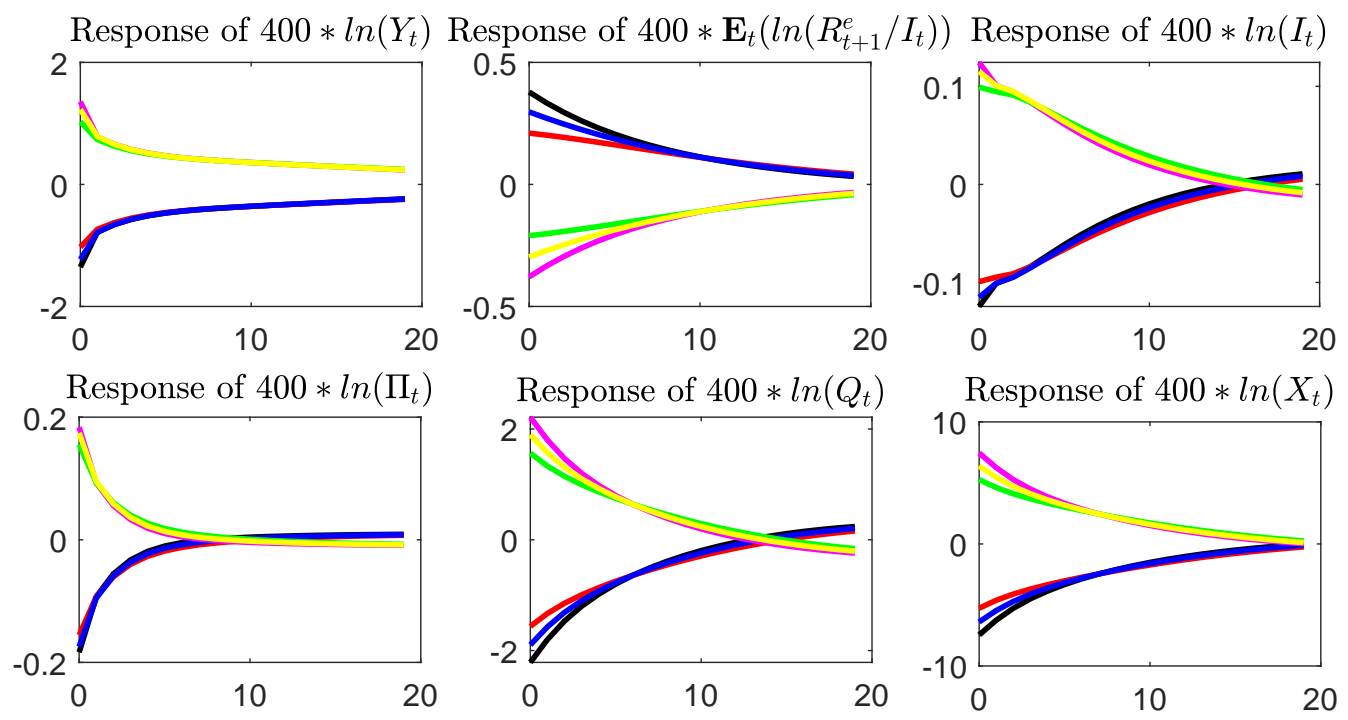

Response of $100 * \Phi\left(\bar{\omega}_{t} \mid \mu_{\omega, t}, \sigma_{\omega, t}\right)$ Response of $100 * N_{t}^{r} / Q_{t} K_{t+1}$
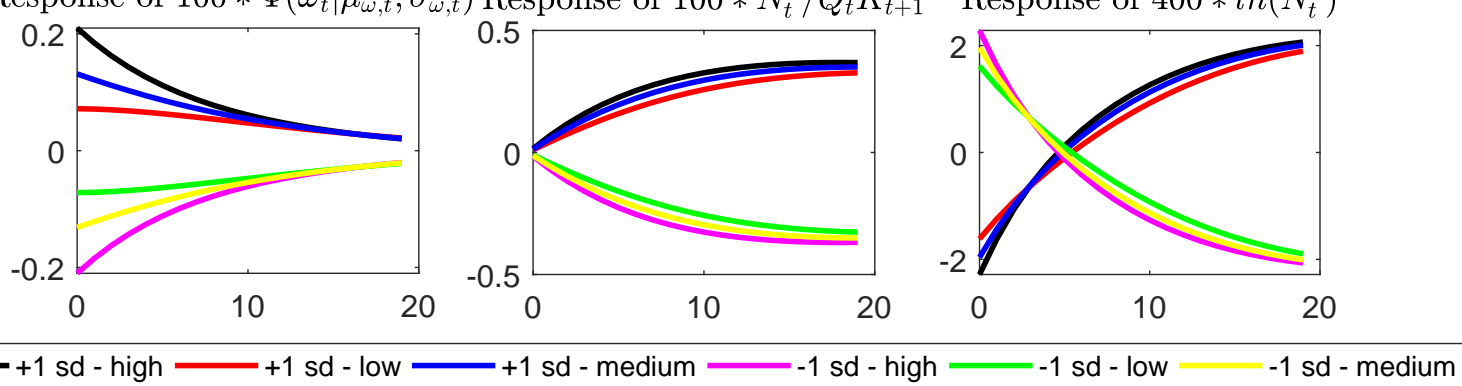
Figure 2D. Generalized Impulse Response Functions: Response to a $+\mathbf{1} / \mathbf{- 1}$ Standard Deviation Interest Rate Rule Shock, Conditional on High, Mid, Low Values of the Endogenous Spread.
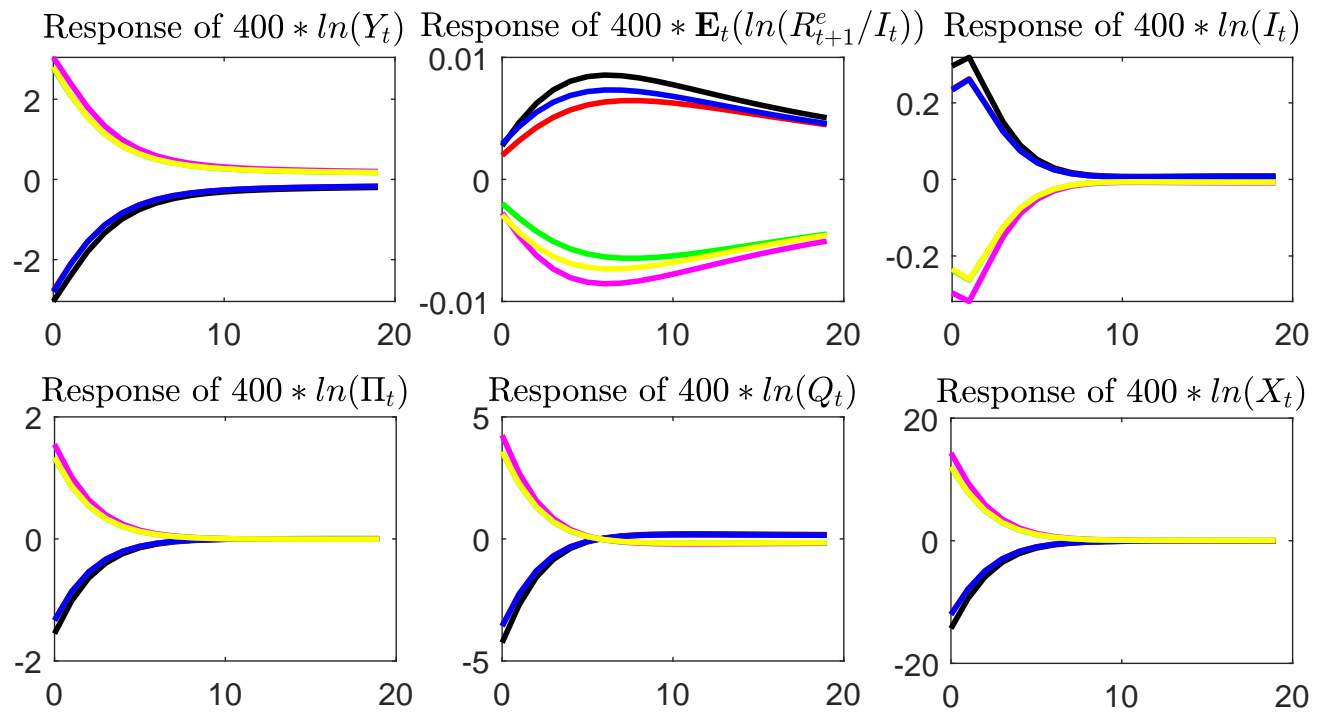

Response of $100 * \Phi\left(\bar{\omega}_{t} \mid \mu_{\omega, t}, \sigma_{\omega, t}\right)$ Response of $100 * N_{t}^{r} / Q_{t} K_{t+1}$
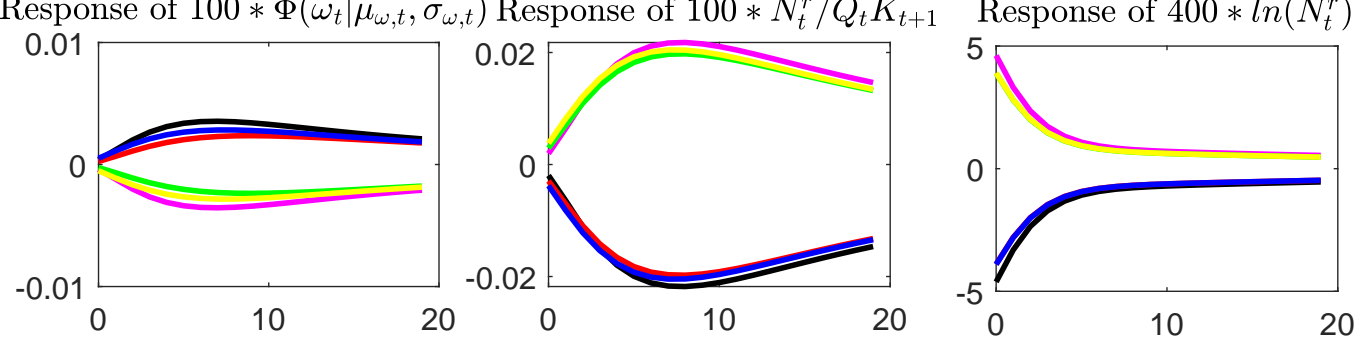

$+1 \mathrm{sd}-$ high $=+1 \mathrm{sd}-$ low $\longrightarrow+1 \mathrm{sd}-$ medium

$-1 \mathrm{sd}$ - high $=-1 \mathrm{sd}-$ low 
Figure 2E. Generalized Impulse Response Functions: Response to a $+1 /-1$ Standard Deviation Interest Rate Rule Stochastic Volatility Shock, Conditional on High, Mid, Low Values of the Endogenous Spread.
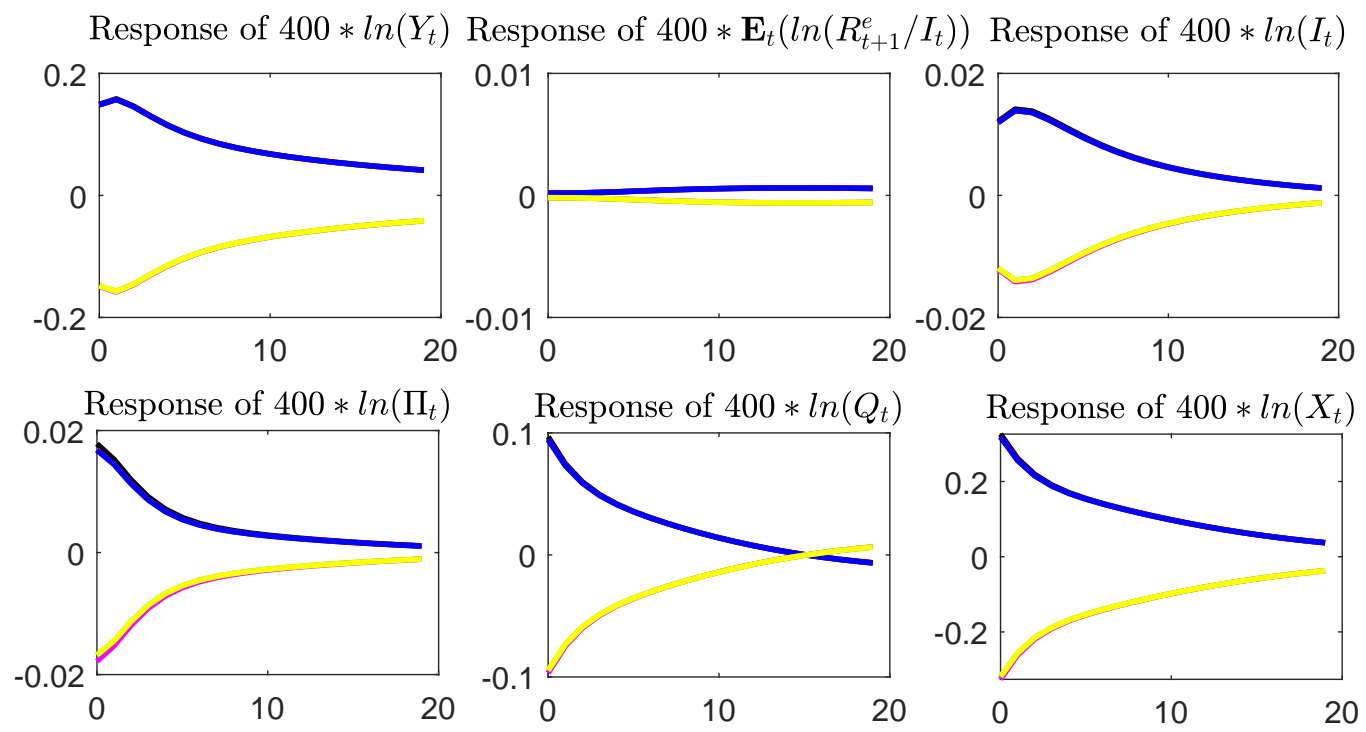

Response of $100 * \Phi\left(\bar{\omega}_{t} \mid \mu_{\omega, t}, \sigma_{\omega, t}\right)$ Response of $100 * N_{t}^{r} / Q_{t} K_{t+1}$
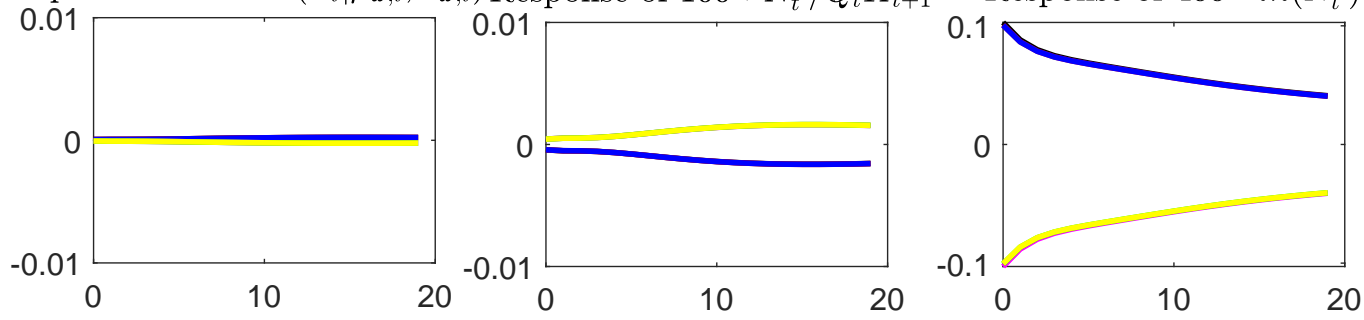

$+1 \mathrm{sd}-\mathrm{high}$

$+1 \mathrm{sd}-$ low

$+1 \mathrm{sd}-$ medium

$-1 \mathrm{sd}-\mathrm{high}=-1 \mathrm{sd}-\mathrm{low}$

$-1 \mathrm{sd}-$ medium 
Figure 3A. Generalized Impulse Response Functions: Response to a $+\mathbf{2} / \mathbf{- 2}$ Standard Deviation TFP Shock, Conditional on High, Mid, Low Values of the Endogenous Spread.
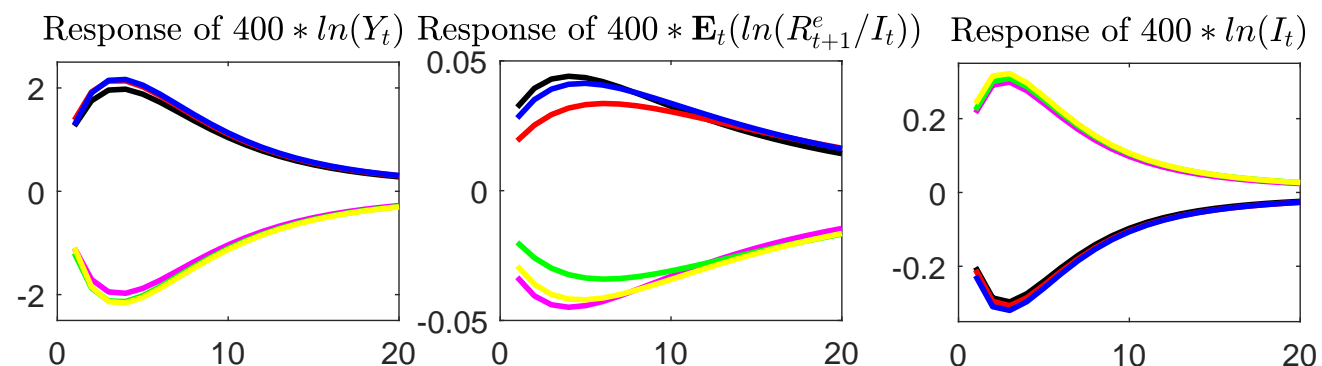

Response of $400 * \ln \left(\Pi_{t}\right)$
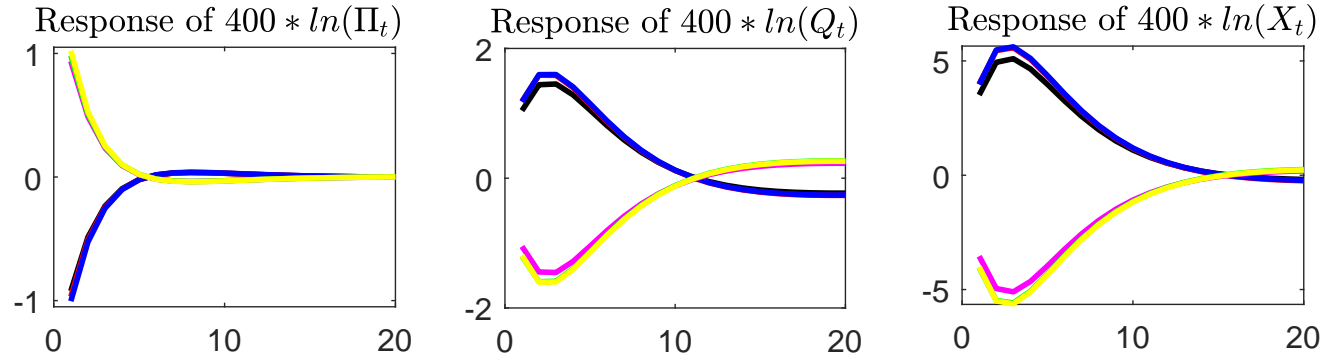

Response of $100 * \Phi\left(\bar{\omega}_{t} \mid \mu_{\omega, t}, \sigma_{\omega, t}\right)$ Response of $100 * N_{t}^{r} / Q_{t} K_{t+1}$
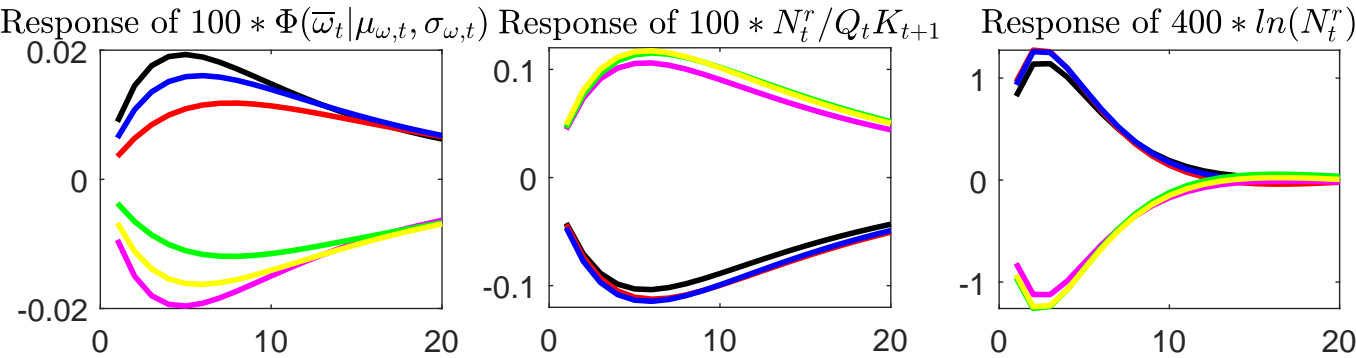

$+2 \mathrm{sd}-$ high $=+2 \mathrm{sd}-$ low

$+2 \mathrm{sd}$ - medium

-2 sd - high

-2 sd - low

$-2 \mathrm{sd}$ - medium 
Figure 3B. Generalized Impulse Response Functions: Response to a +2/-2 Standard Deviation TFP Stochastic Volatility Shock, Conditional on High, Mid, Low Values of the Endogenous Spread.
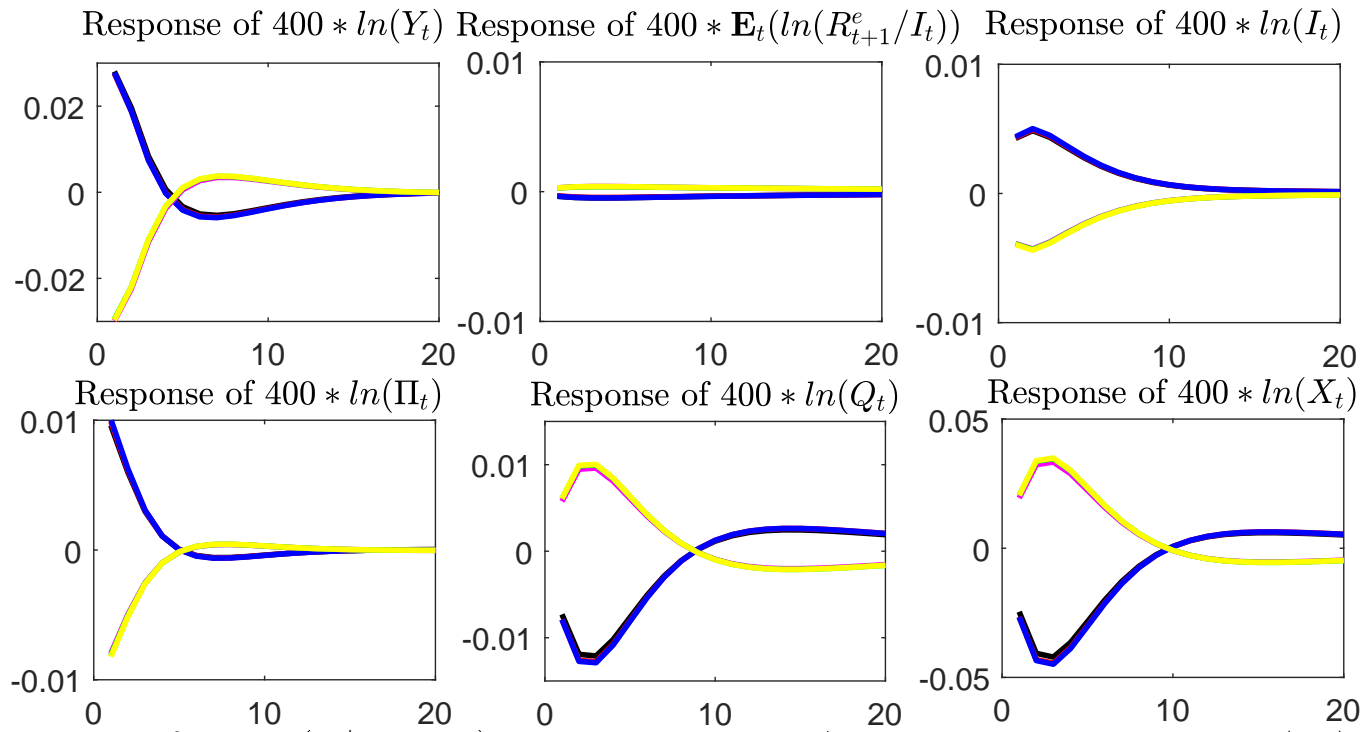

Response of $100 * \Phi\left(\bar{\omega}_{t} \mid \mu_{\omega, t}, \sigma_{\omega, t}\right)$ Response of $100 * N_{t}^{r} / Q_{t} K_{t+1} \quad$ Response of $400 * \ln \left(N_{t}^{r}\right)$
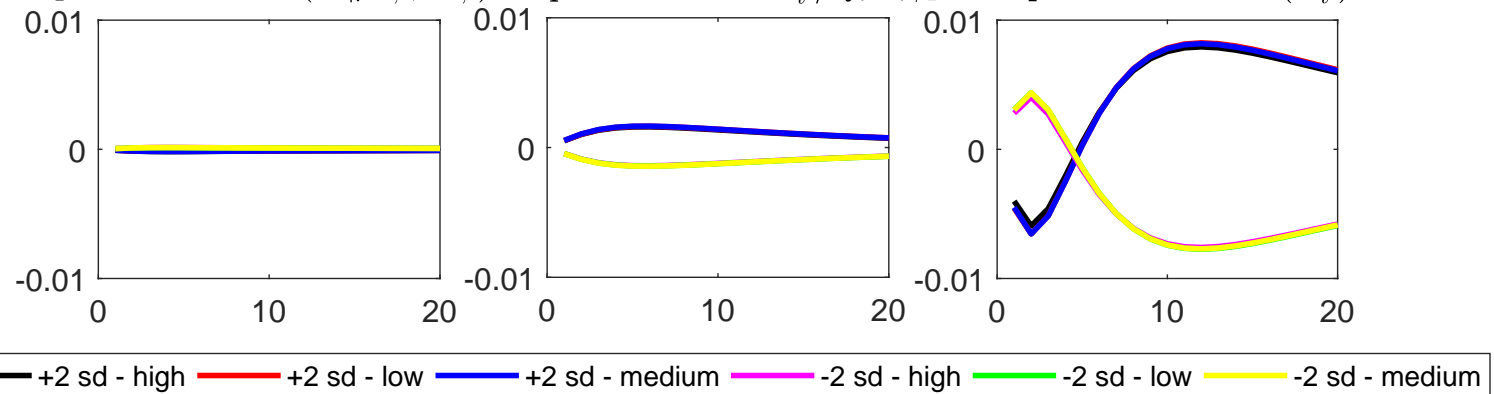
Figure 3C. Generalized Impulse Response Functions: Response to a $+2 /-2$ Standard Deviation Micro-Uncertainty Shock, Conditional on High, Mid, Low Values of the Endogenous Spread.
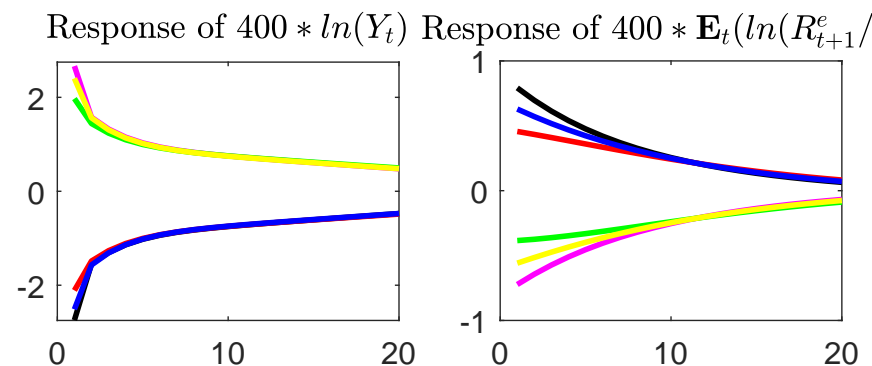

$\left.\left.{ }_{-1} / I_{t}\right)\right) \quad$ Response of $400 * \ln \left(I_{t}\right)$
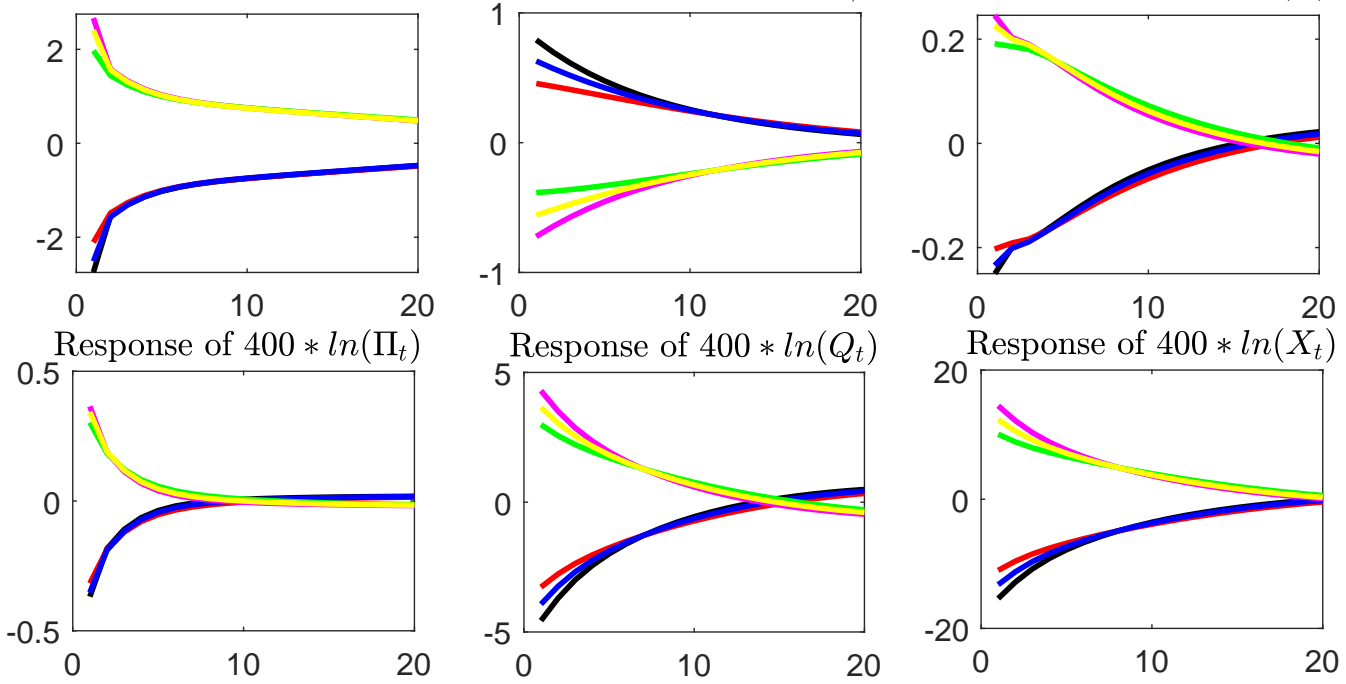

Response of $100 * \Phi\left(\bar{\omega}_{t} \mid \mu_{\omega, t}, \sigma_{\omega, t}\right)$ Response of $100 * N_{t}^{r} / Q_{t} K_{t+1}$
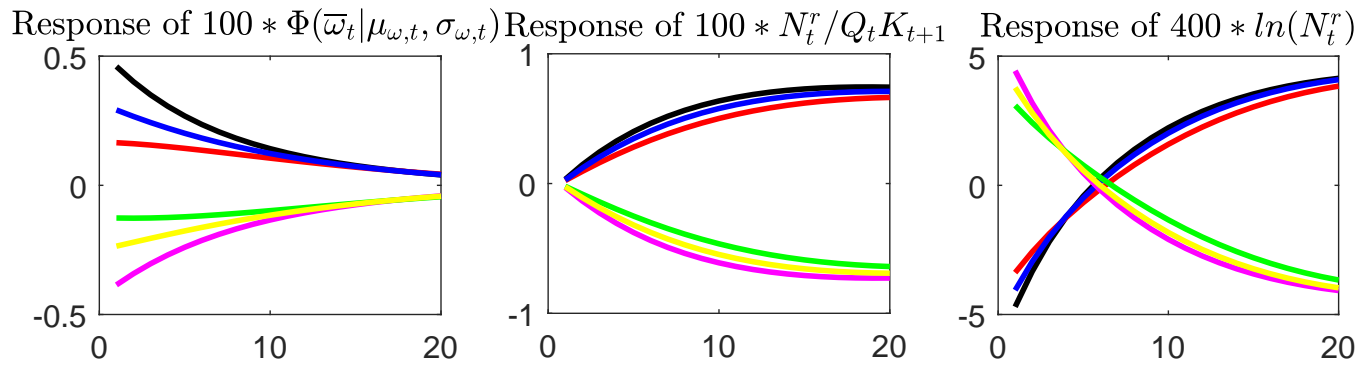

+2 sd - high +2 sd - low

+2 sd - medium

-2 sd - high

-2 sd - low

-2 sd - medium 
Figure 3D. Generalized Impulse Response Functions: Response to a $+\mathbf{2} /-2$ Standard Deviation Interest Rate Rule Shock, Conditional on High, Mid, Low Values of the Endogenous Spread.
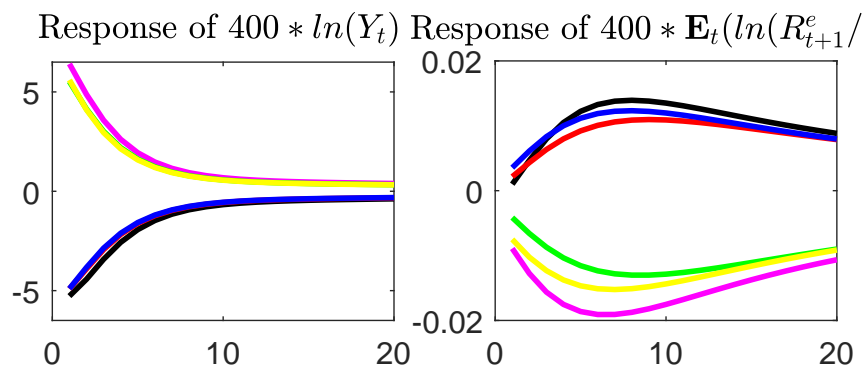

Response of $400 * \ln \left(\Pi_{t}\right)$
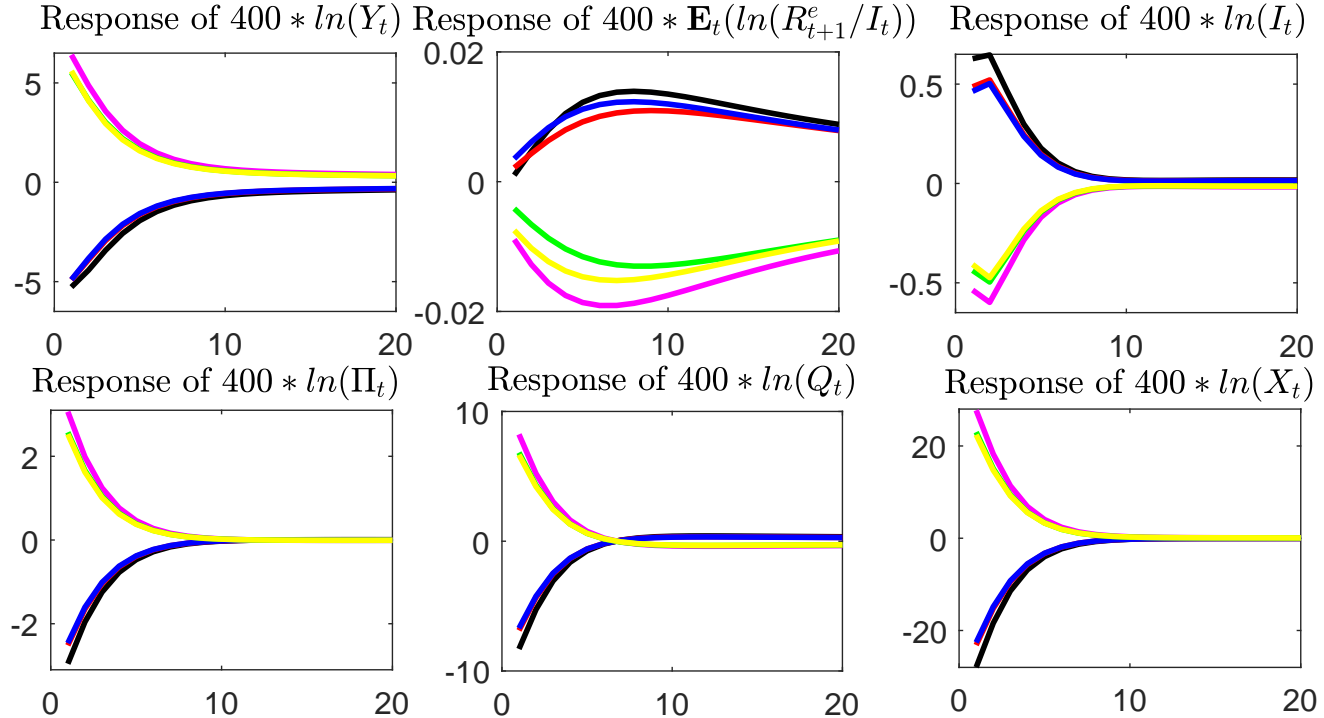

Response of $100 * \Phi\left(\bar{\omega}_{t} \mid \mu_{\omega, t}, \sigma_{\omega, t}\right.$
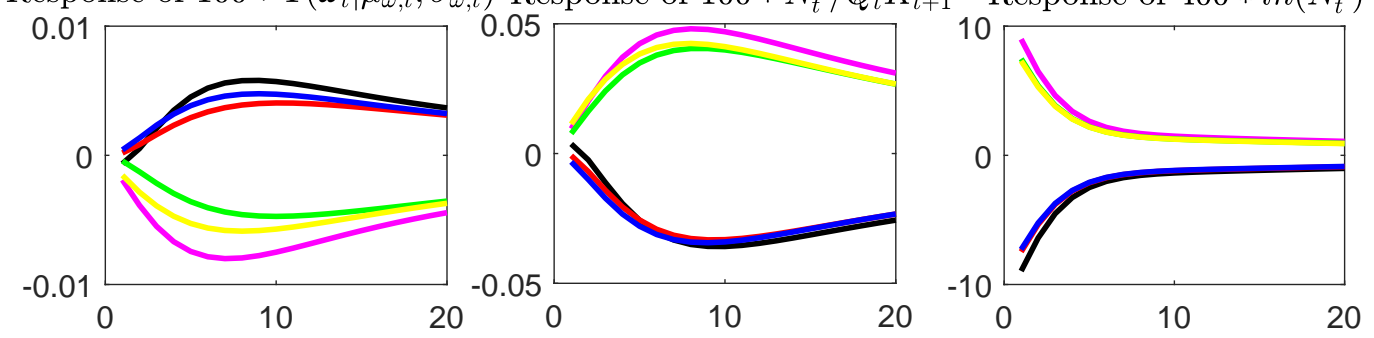

$+2 \mathrm{sd}$ - high $\longrightarrow+2 \mathrm{sd}-$ low $\longrightarrow+2 \mathrm{sd}-$ medium $\longrightarrow-2 \mathrm{sd}-$ high

-2 sd - low

$-2 \mathrm{sd}-$ medium 
Figure 3E. Generalized Impulse Response Functions: Response to a +2/-2 Standard Deviation Interest Rate Rule Stochastic Volatility Shock, Conditional on High, Mid, Low Values of the Endogenous Spread.

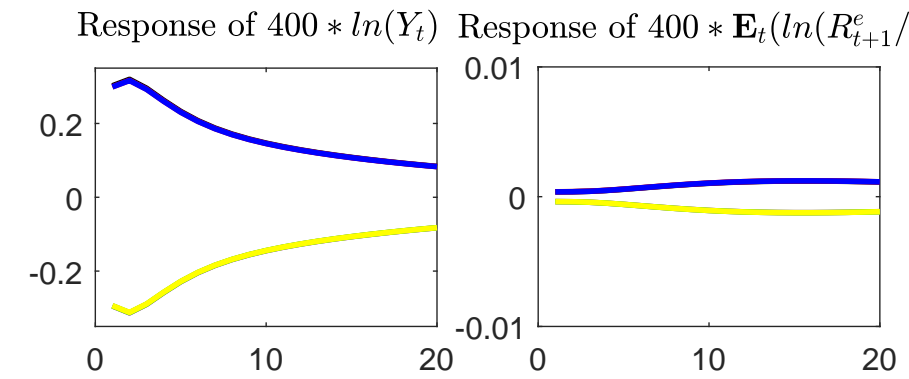

$\left.I_{t}\right)$ ) Response of $400 * \ln \left(I_{t}\right)$

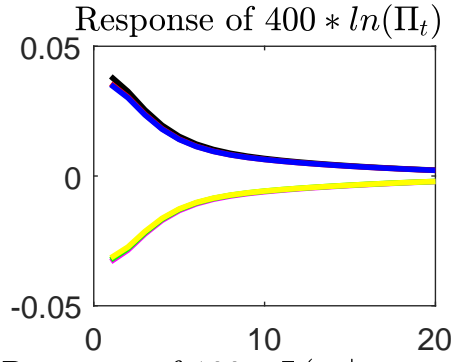

Response of $400 * \ln \left(Q_{t}\right)$
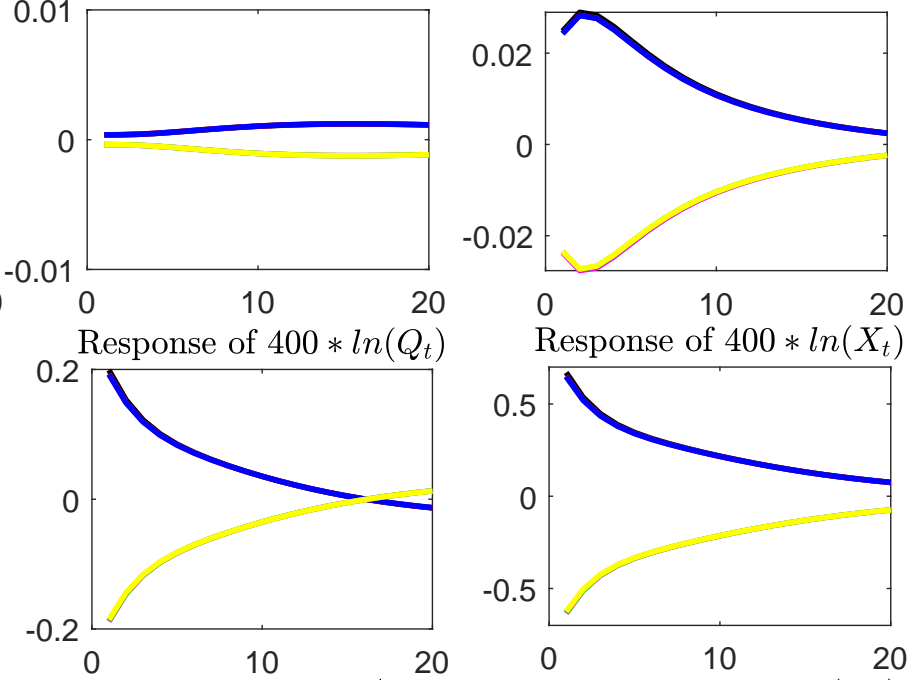

Response of $100 * \Phi\left(\bar{\omega}_{t} \mid \mu_{\omega, t}, \sigma_{\omega, t}\right)$ Response of $100 * N_{t}^{r} / Q_{t} K_{t+1}$
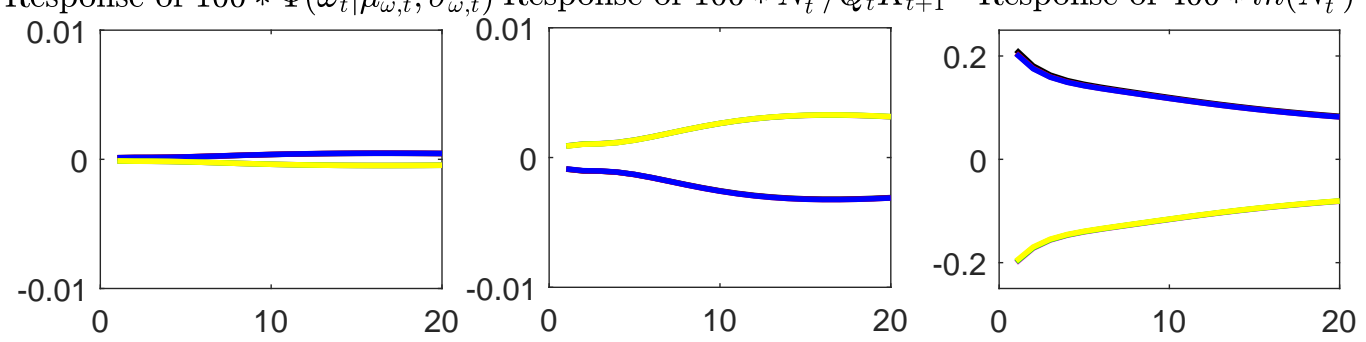

$+2 \mathrm{sd}-$ high $-+2 \mathrm{sd}-$ low

$+2 \mathrm{sd}-$ medium $--2 \mathrm{sd}-\mathrm{high}$

-2 sd - low

-2 sd - medium 
Figure 4A. Generalized Impulse Response Functions: Response to a +1 Standard Deviation TFP Shock, Conditional on the Level of Micro-Uncertainty.
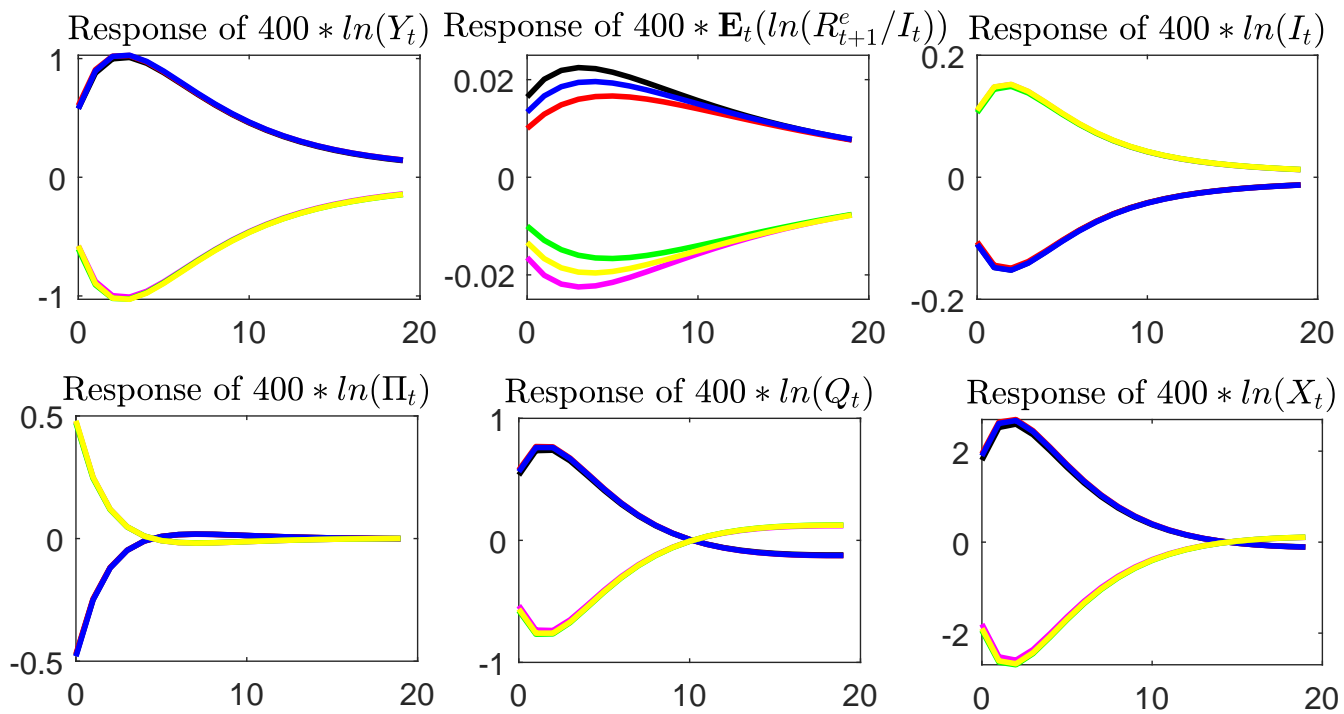

Response of $400 * \ln \left(X_{t}\right)$
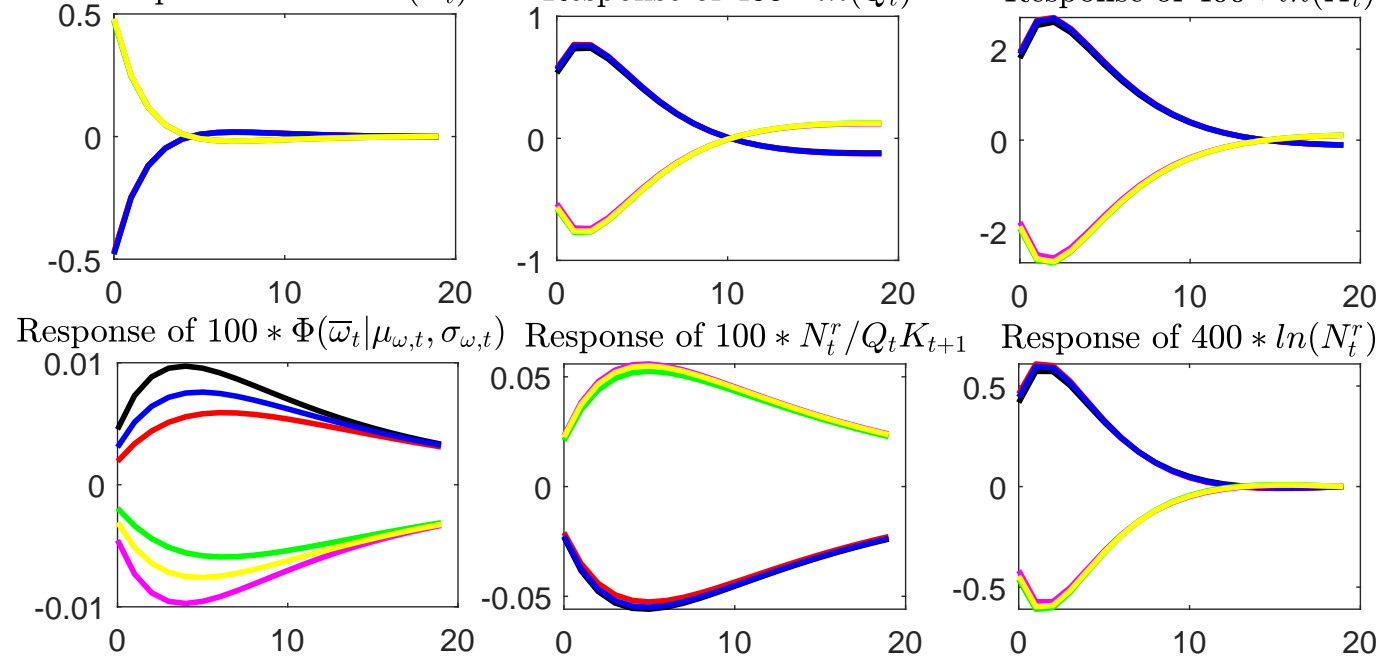
Figure 4B. Generalized Impulse Response Functions: Response to a +1 Standard Deviation TFP Stochastic Volatility Shock, Conditional on the Level of Micro-Uncertainty.
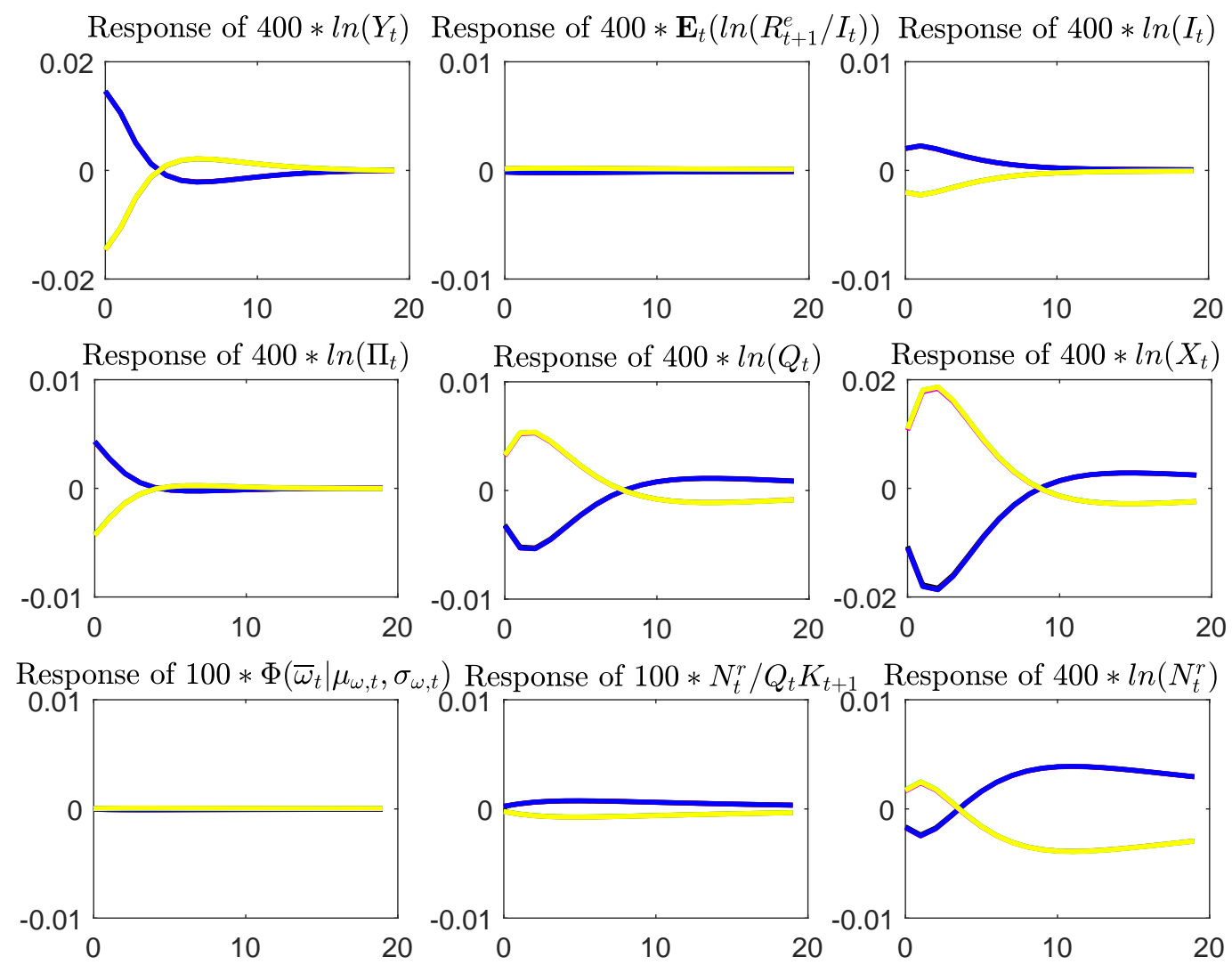

$+1 \mathrm{sd}-$ high $=+1 \mathrm{sd}-$ low

$+1 \mathrm{sd}-$ medium

-1 sd - high

$-1 \mathrm{sd}-$ low

$-1 \mathrm{sd}$ - medium 
Figure 4C. Generalized Impulse Response Functions: Response to a +1 Standard Deviation Micro-Uncertainty Shock, Conditional on the Level of Micro-Uncertainty.
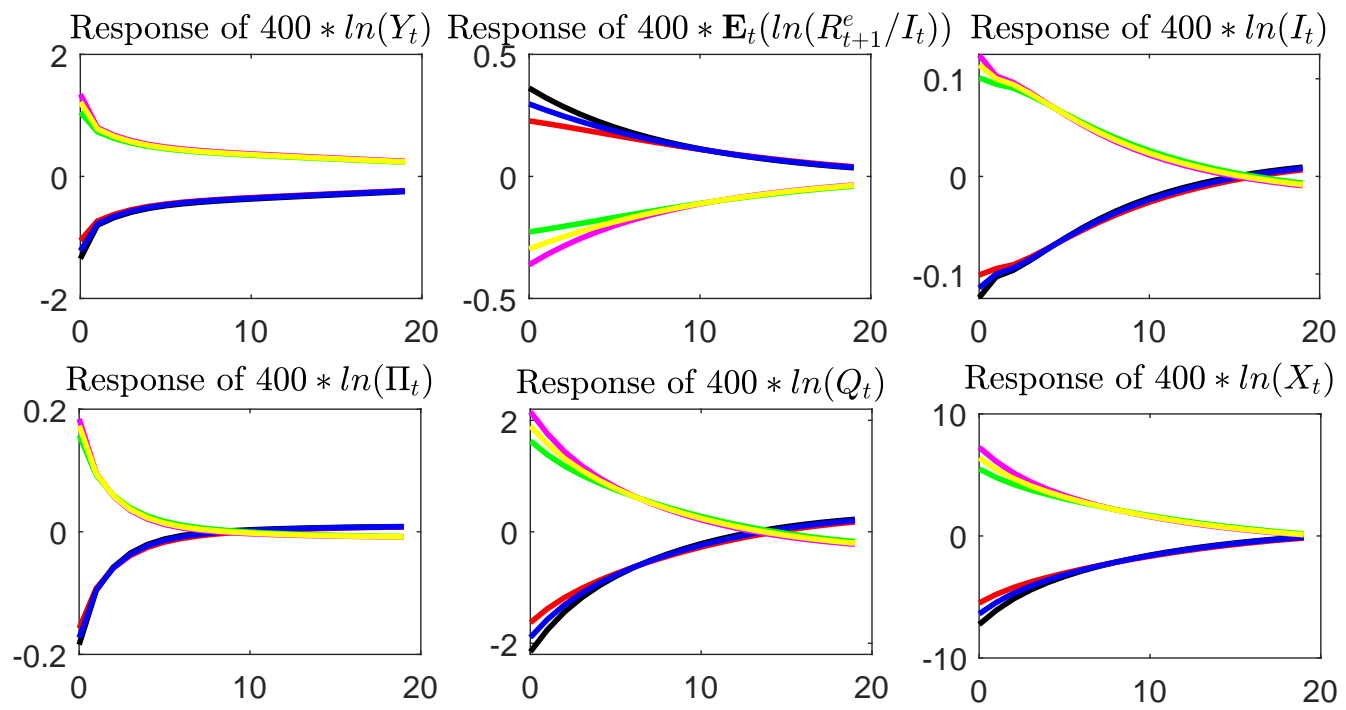

Response of $100 * \Phi\left(\bar{\omega}_{t} \mid \mu_{\omega, t}, \sigma_{\omega, t}\right)$ Response of $100 * N_{t}^{r} / Q_{t} K_{t+1} \quad$ Response of $400 * \ln \left(N_{t}^{r}\right)$
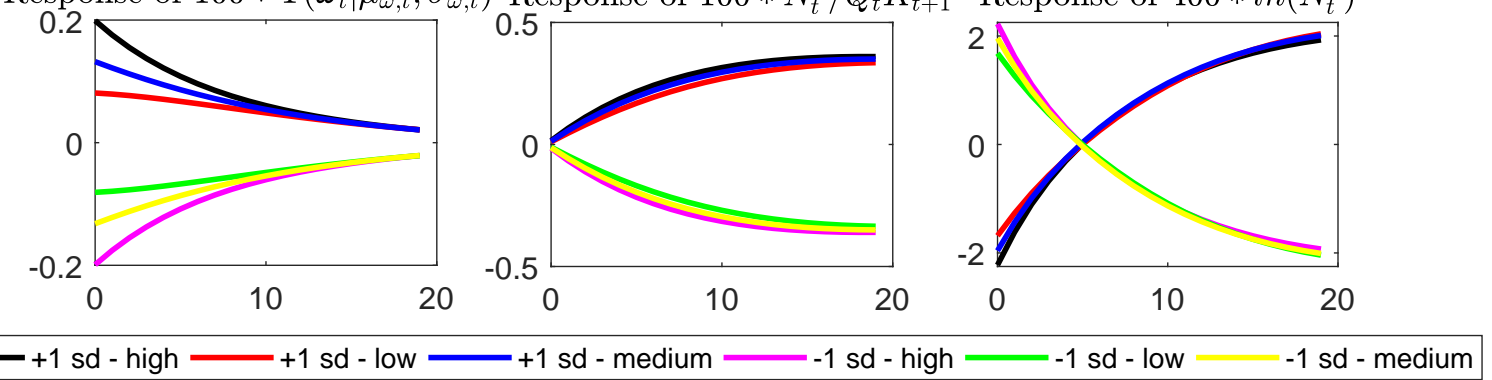
Figure 4D. Generalized Impulse Response Functions: Response to a +1 Standard Deviation Interest Rate Rule Shock, Conditional on the Level of Micro-Uncertainty.
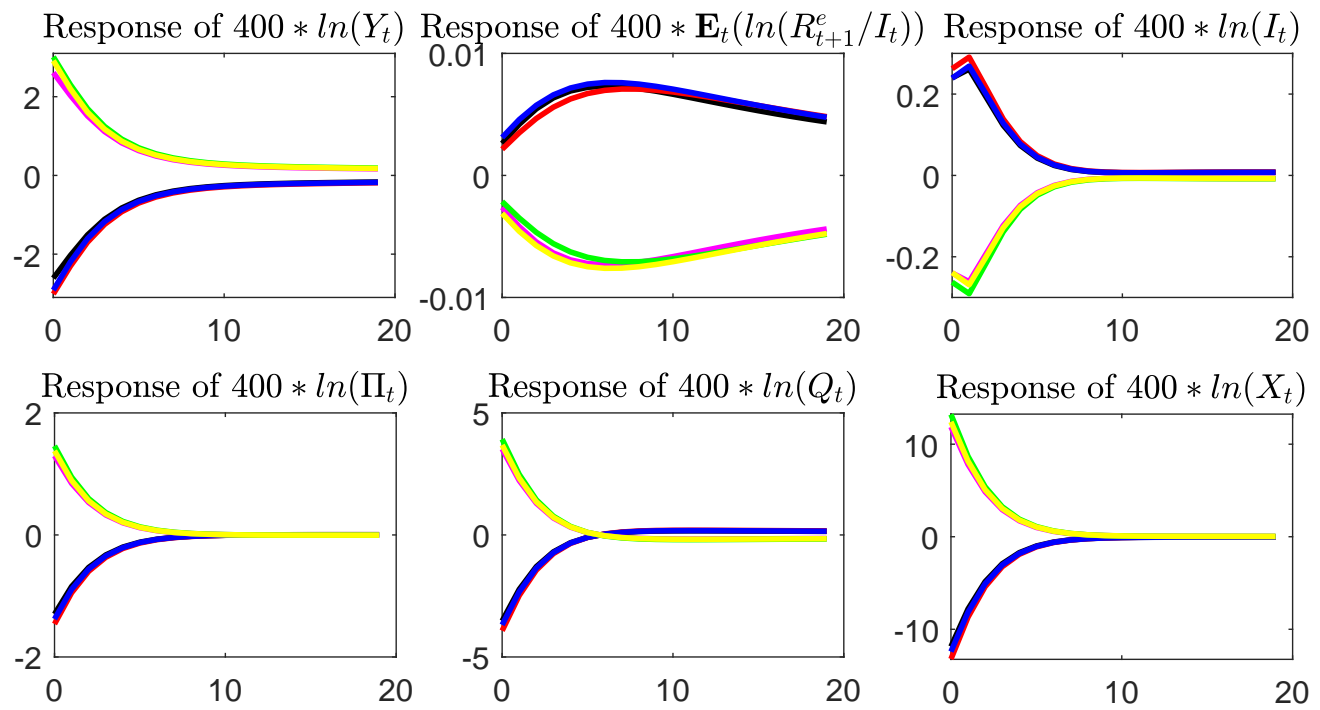

Response of $100 * \Phi\left(\bar{\omega}_{t} \mid \mu_{\omega, t}, \sigma_{\omega, t}\right)$
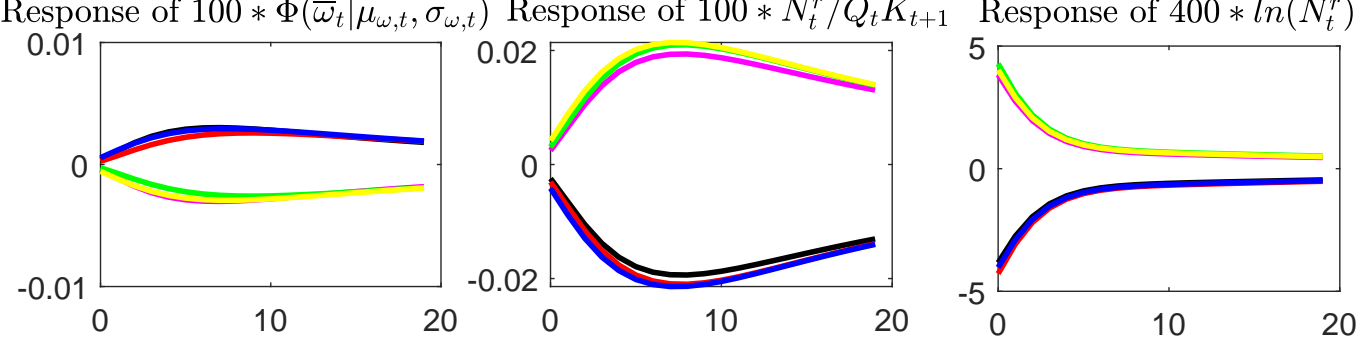
Figure 4E. Generalized Impulse Response Functions: Response to a +1 Standard Deviation Interest Rate Rule Stochastic Volatility Shock, Conditional on the Level of Micro-Uncertainty.
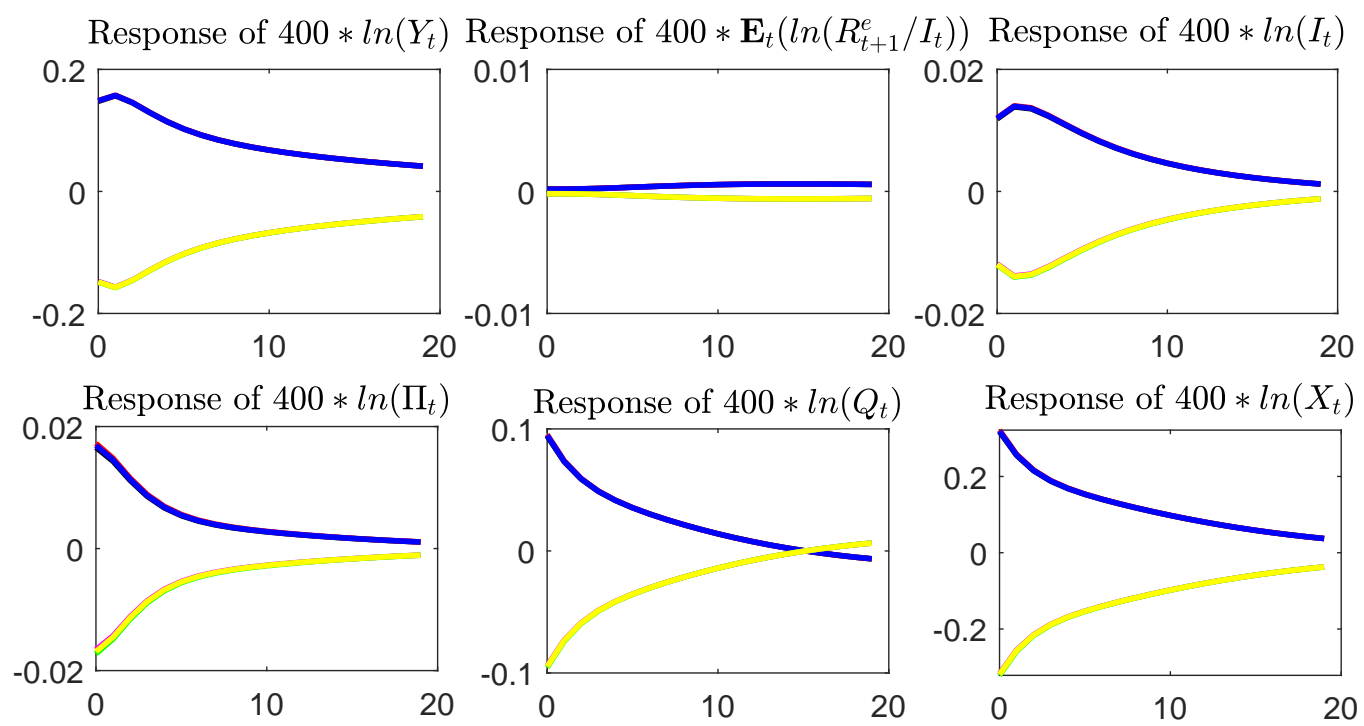

Response of $100 * \Phi\left(\bar{\omega}_{t} \mid \mu_{\omega, t}, \sigma_{\omega, t}\right)$ Response of $100 * N_{t}^{r} / Q_{t} K_{t+1} \quad$ Response of $400 * \ln \left(N_{t}^{r}\right)$
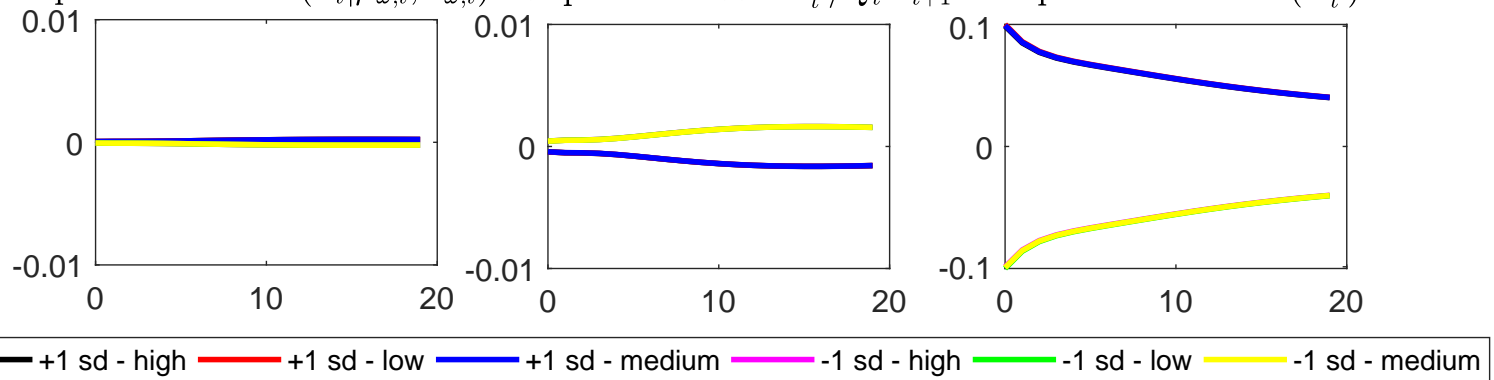
Figure 5A. Generalized Impulse Response Functions: Response to a +1 Shock, Initial Condition: Unconditional Mean.

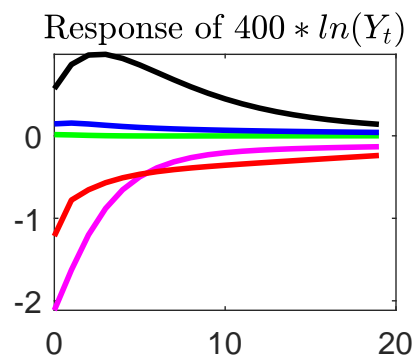

Response of $400 * \ln \left(X_{t}\right)$
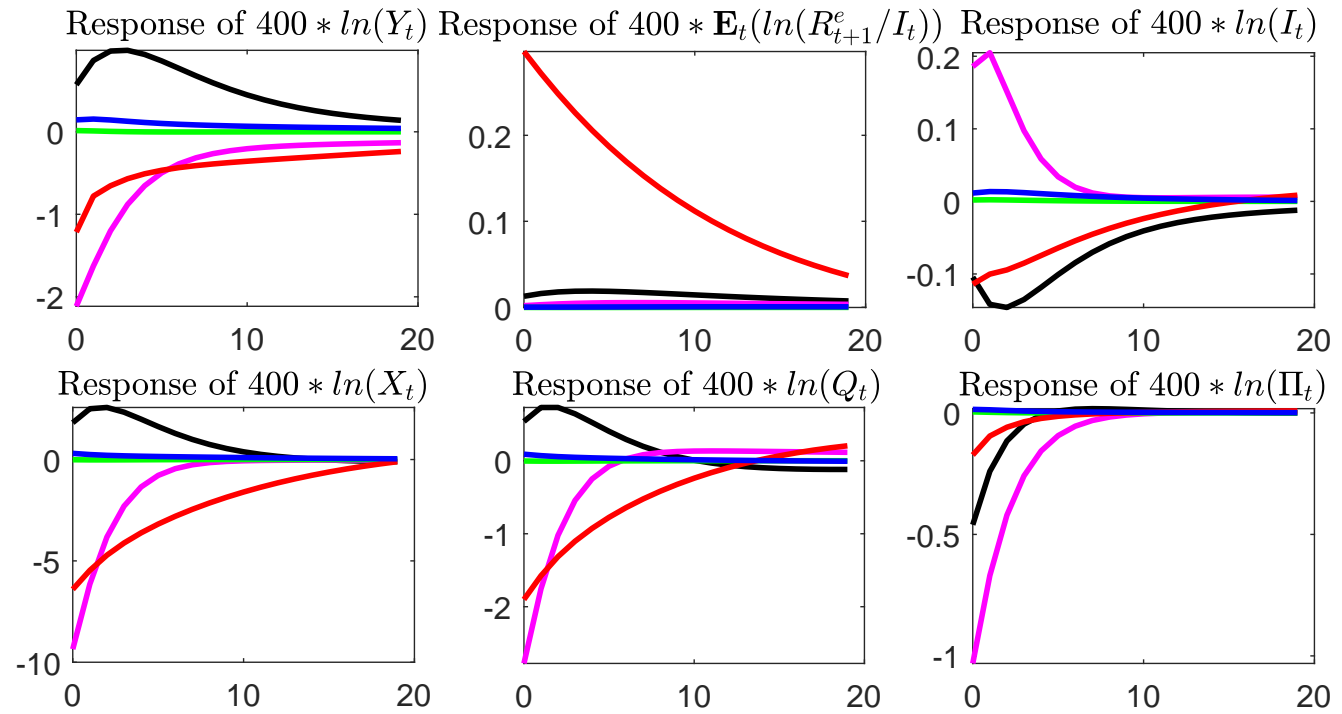

Response of $400 * \ln \left(Q_{t}\right)$
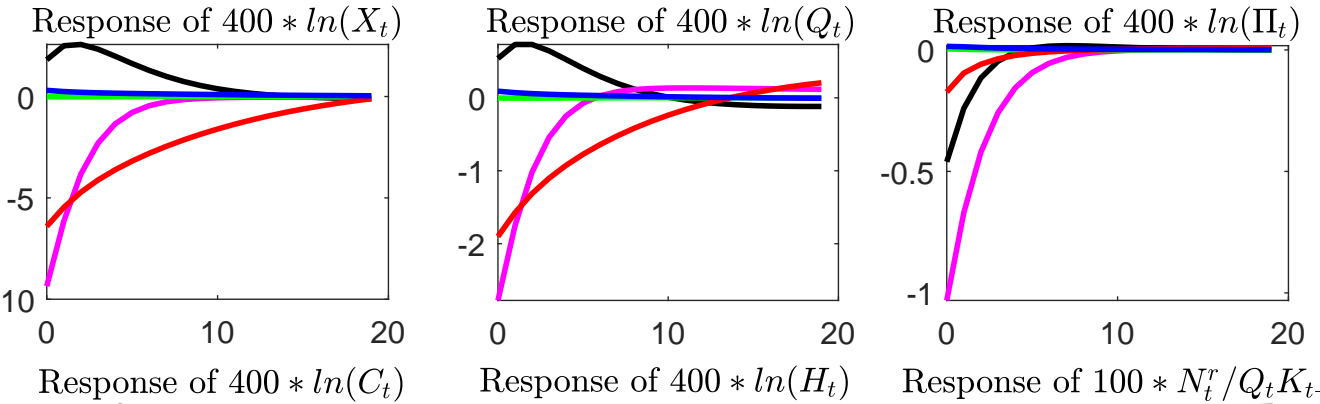

Response of $400 * \ln \left(H_{t}\right)$
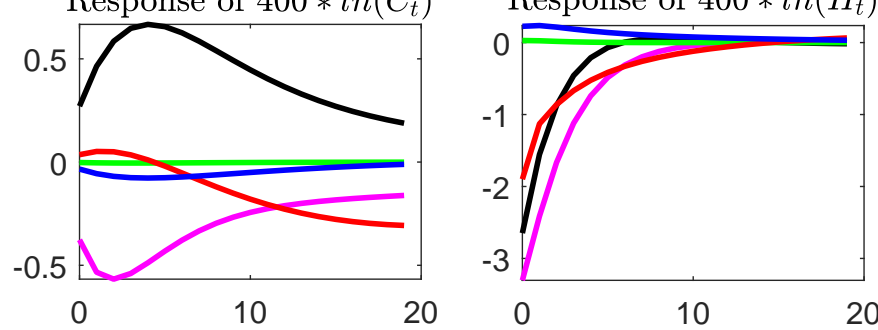

Response of $100 * N_{t}^{r} / Q_{t} K_{t+1}$

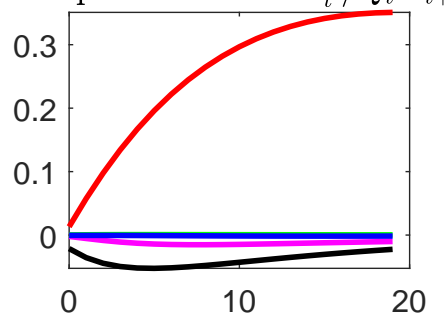

TFP Monetary policy TFP uncertainty Monetary uncertainty 
Figure 5B. Generalized Impulse Response Functions: Response to a +1 Shock, Initial Condition: Conditional on 5th and 95th Percentiles on Spread.
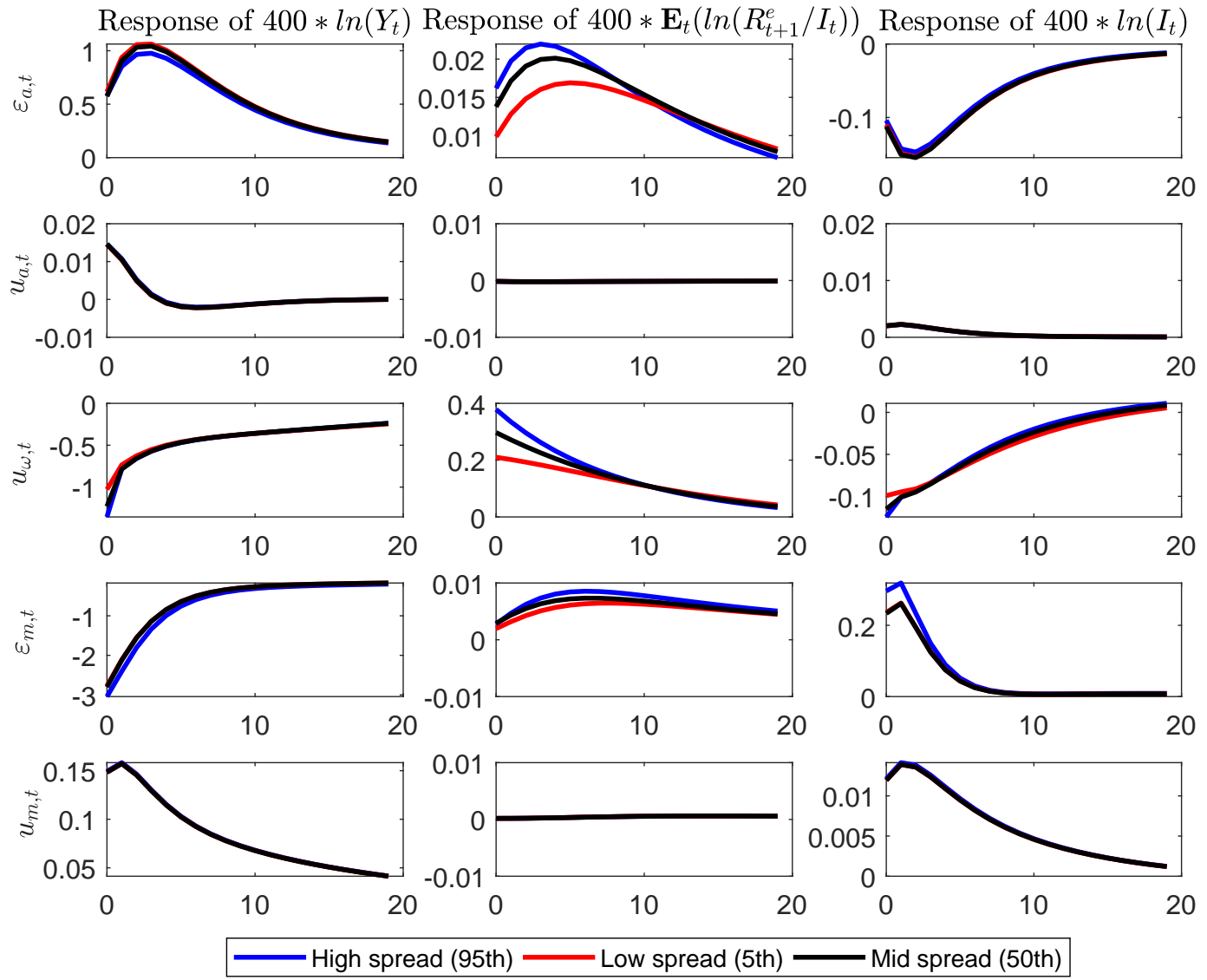
Figure 5C. Generalized Impulse Response Functions: Response to a +1 Shock, Initial Condition: Conditional on 1st and 99th Percentiles on Spread.
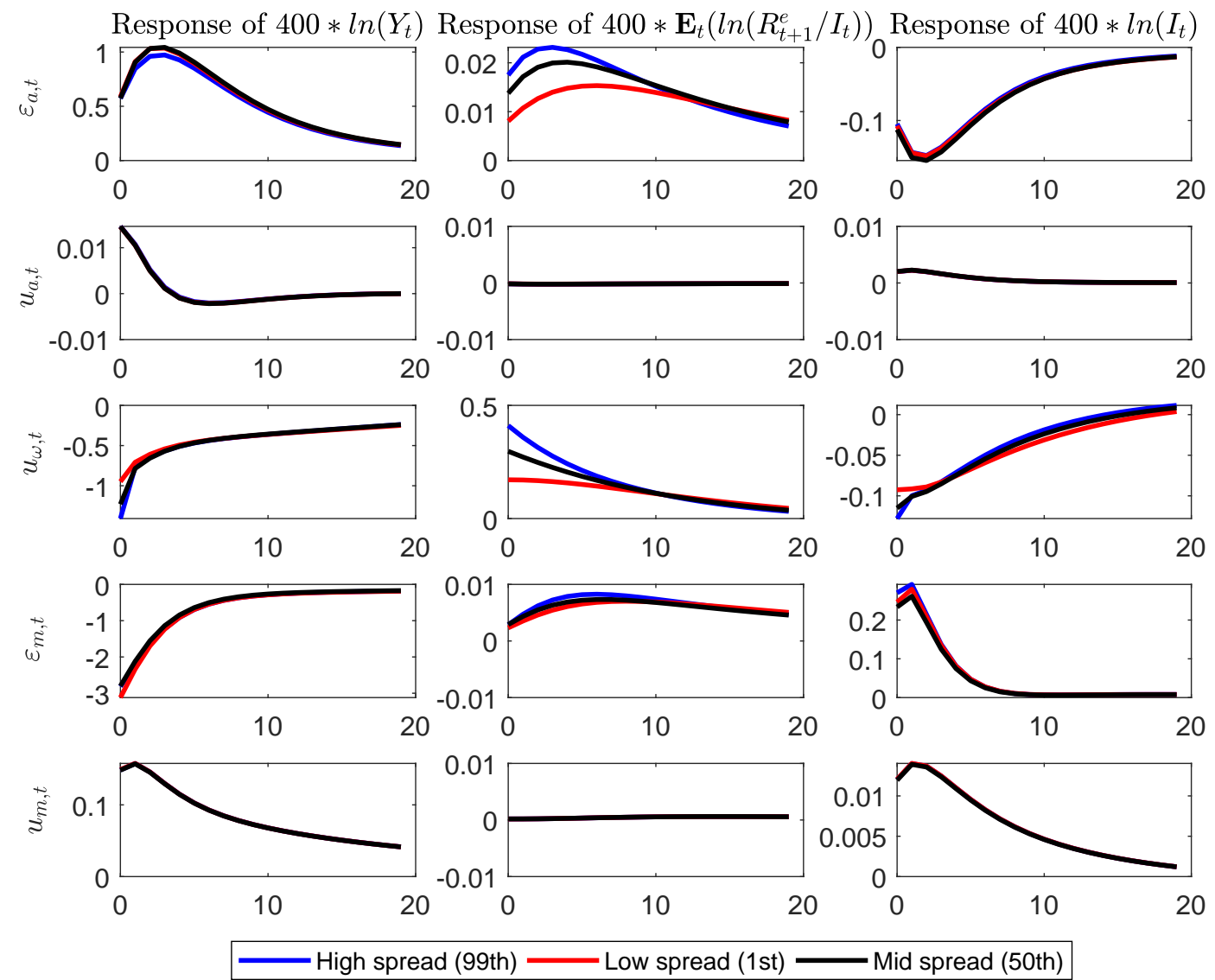
Figure 6A. Generalized Impulse Response Functions: Response to a +1 Shock, For Alternative Model Specifications, Conditional on High Spreads.
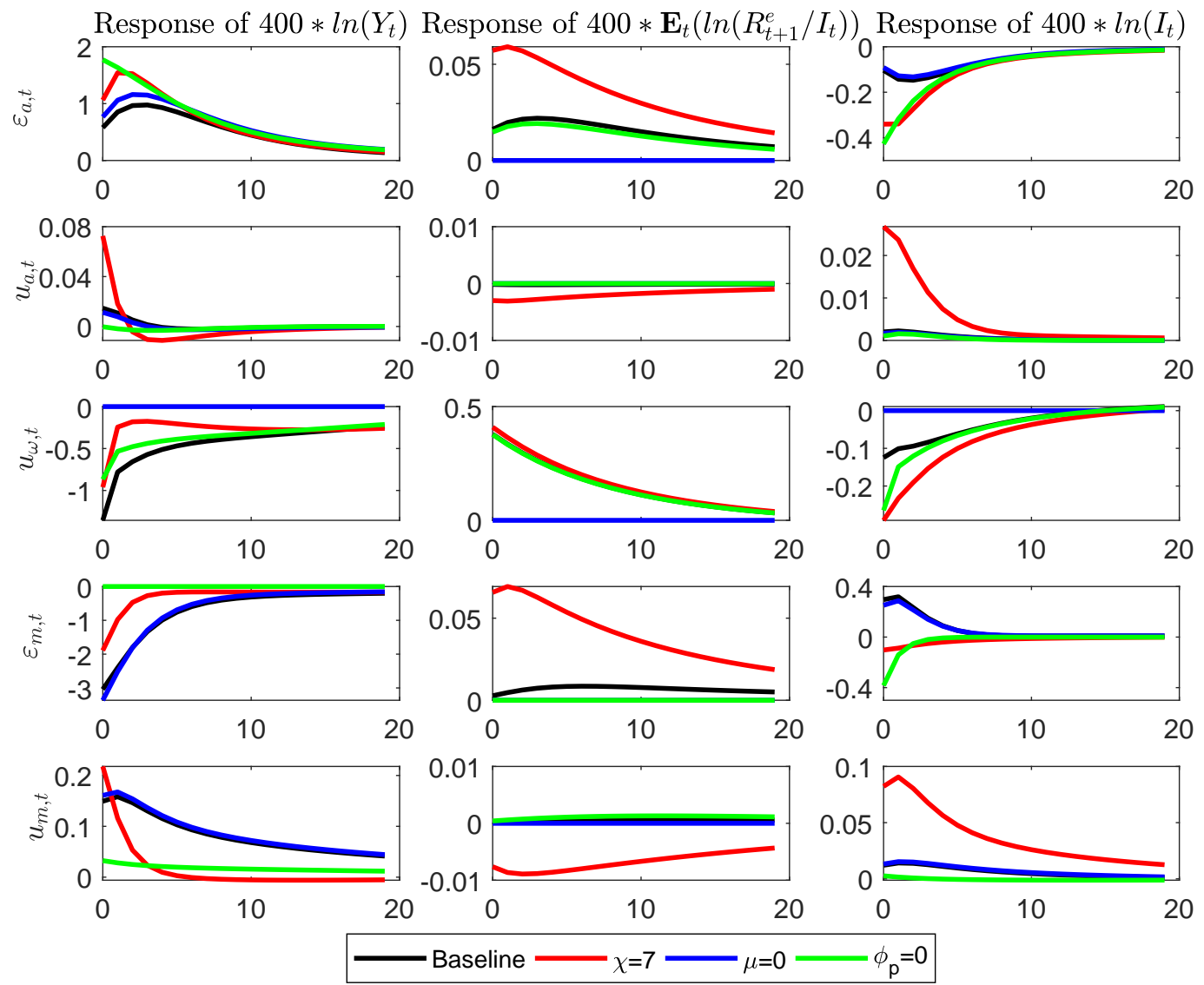
Figure 6B. Generalized Impulse Response Functions: Response to a +1 Shock, Conditional on High and Low Spreads, With and Without Micro-Uncertainty.
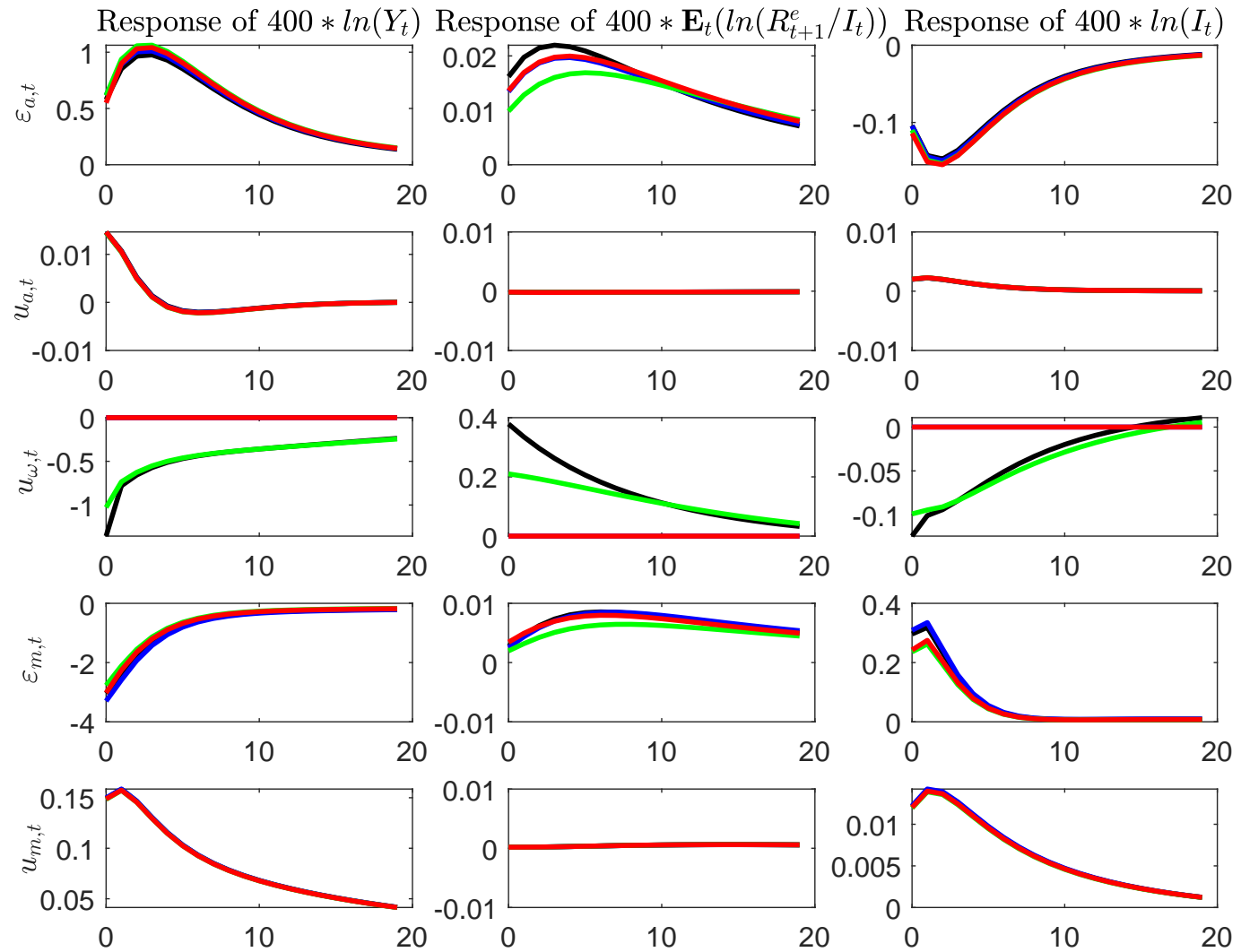

- High spread — High spread, no micro uncert. —— Low spread — Low spread, no micro uncert.

- High spread - High spread, no micro uncert. $—$ Low spread — Low spread, no micro uncert.

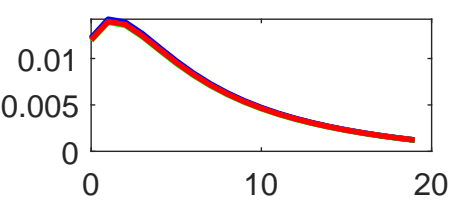


Figure 6C. Generalized Impulse Response Functions: Response to a +1 Shock, Conditional on High Uncertainty (Individually).
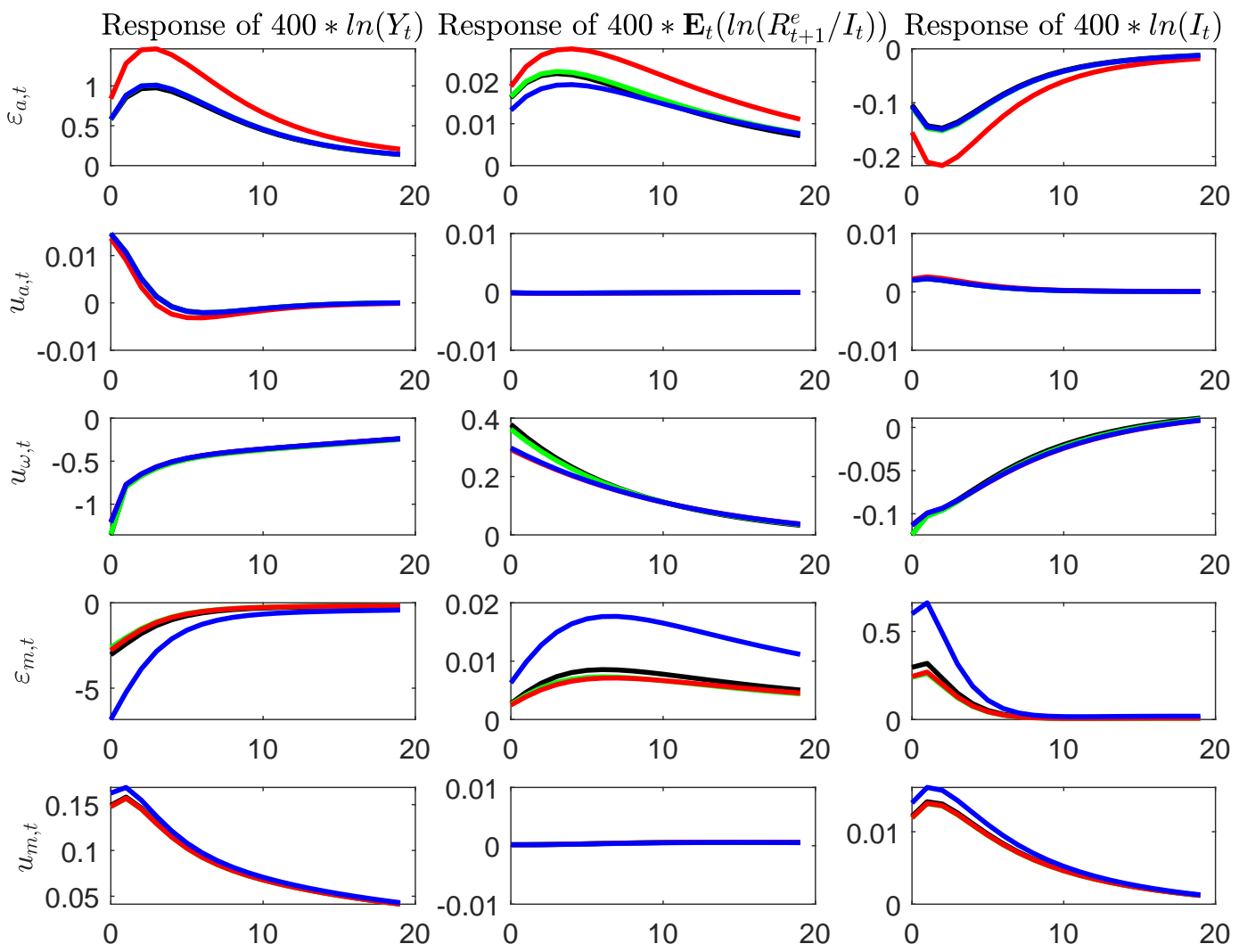

High spread $=$ High micro uncertainty $=$ High TFP uncertainty

High monetary uncertainty 
Figure 6D. Generalized Impulse Response Functions: Response to a +1 Shock, When All Three Stochastic Volatility Shocks are High or Low Simultaneously ( $>$ 80th Percentile or $<$ 20th Percentile).
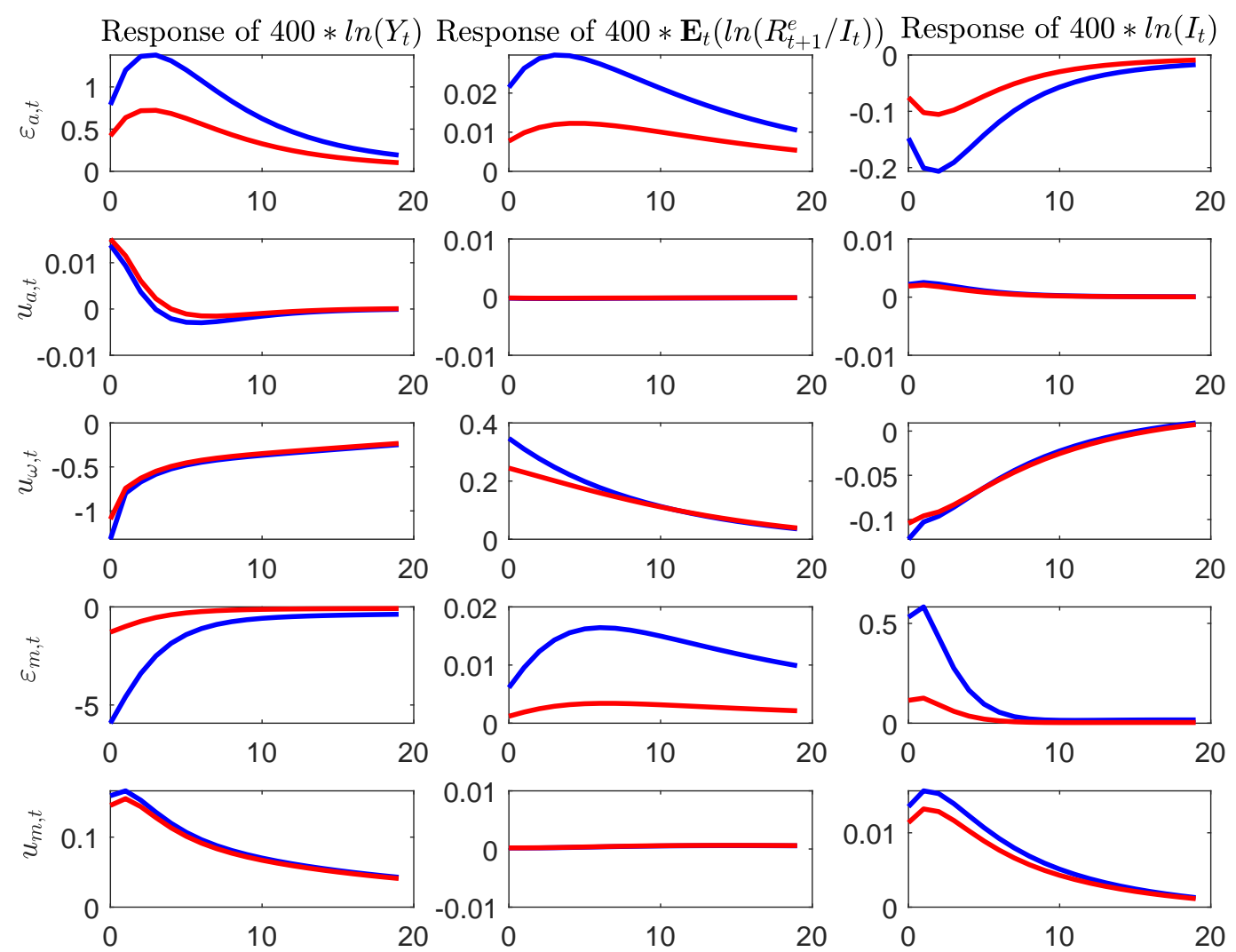

High uncertainty — Low uncertainty 
Figure 7A. Generalized Impulse Response Functions: Response to a +1 Standard Deviation TFP Shock, Various Model Specifications, Conditional on Mid-Values of the Endogenous Spread.
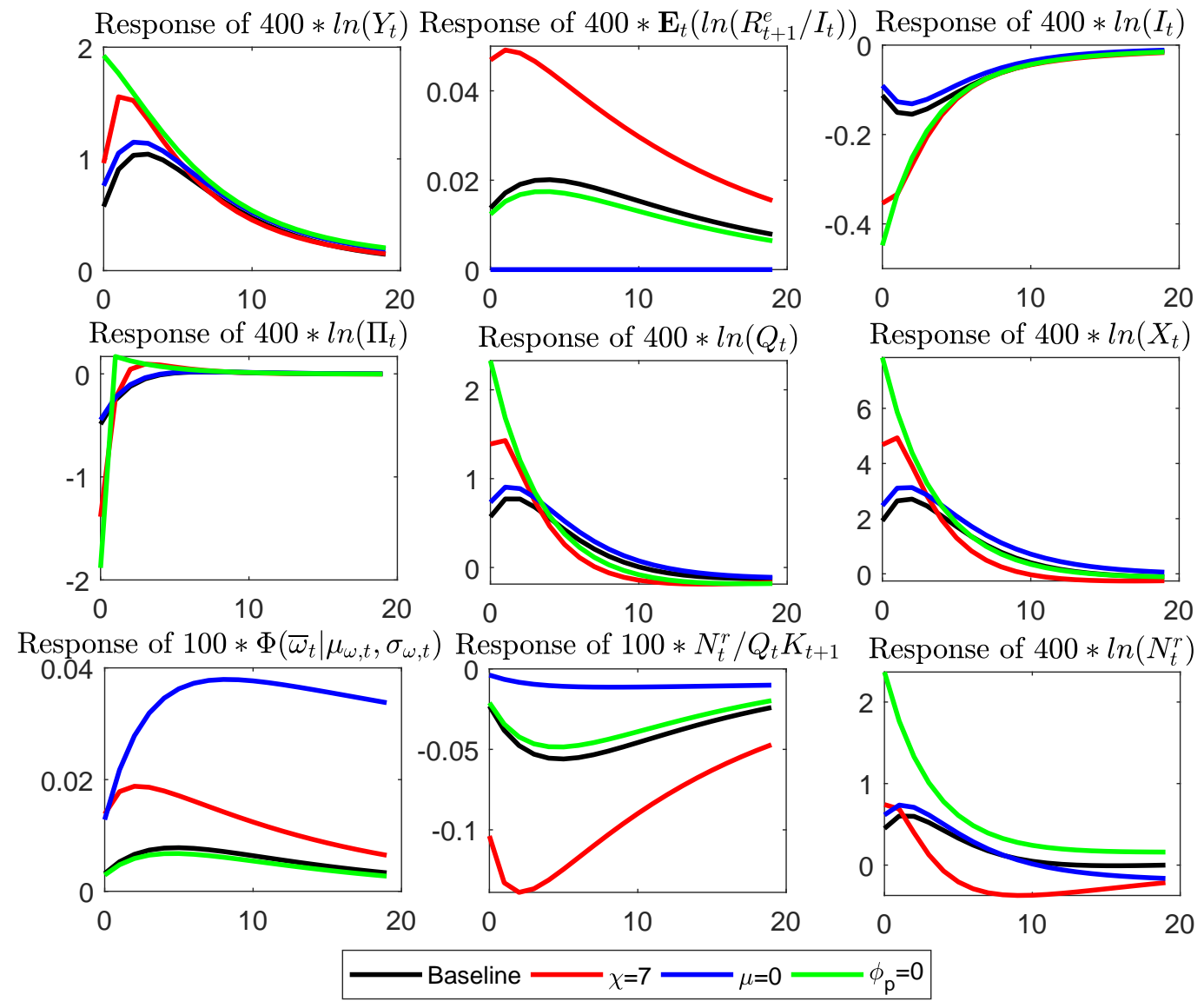
Figure 7B. Generalized Impulse Response Functions: Response to a +1 Standard Deviation TFP Stochastic VolatilityShock, Various Model Specifications, Conditional on Mid-Values of the Endogenous Spread.
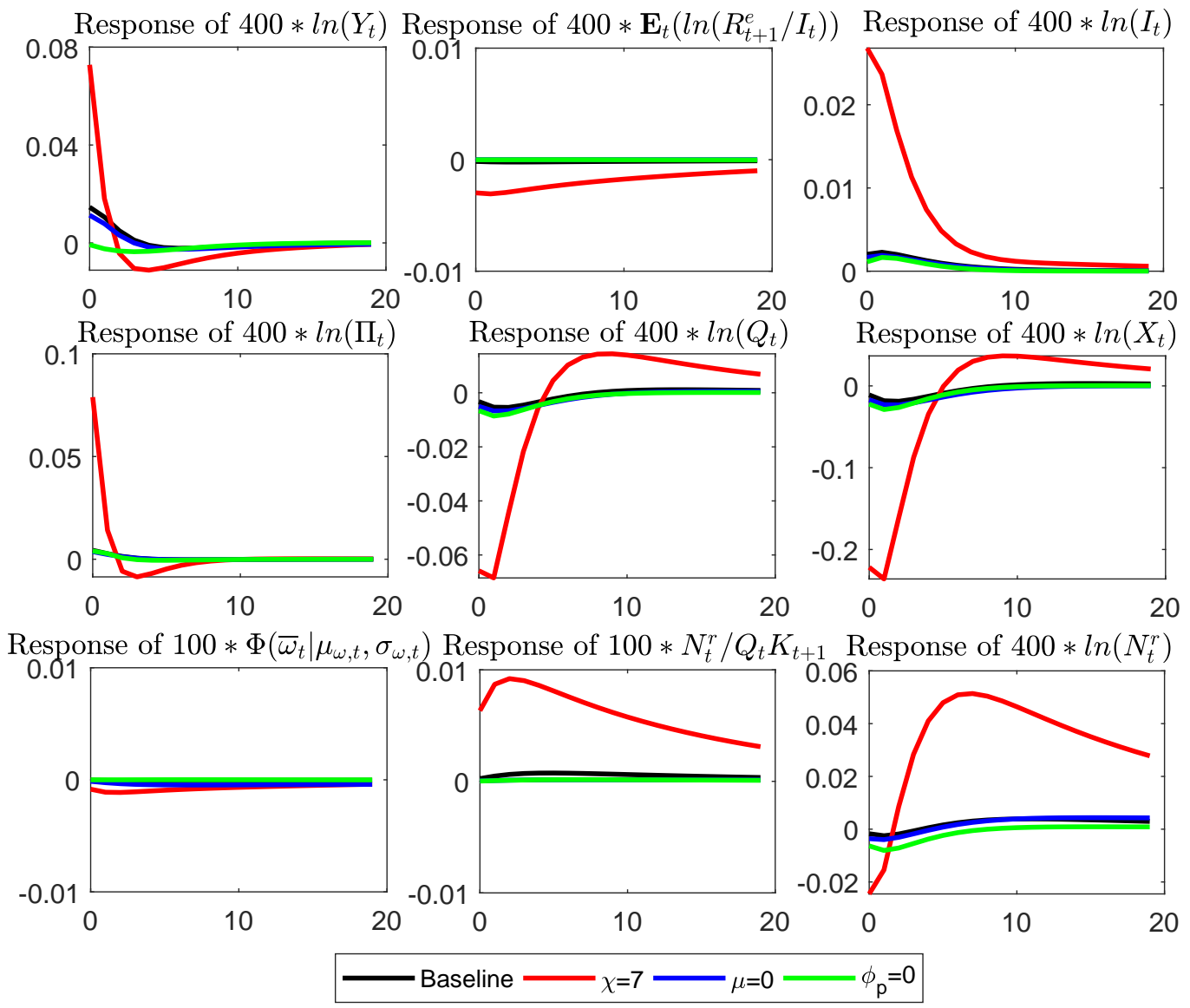
Figure 7C. Generalized Impulse Response Functions: Response to a +1 Standard Deviation Micro-Uncertainty Shock, Various Model Specifications, Conditional on Mid-Values of the Endogenous Spread.
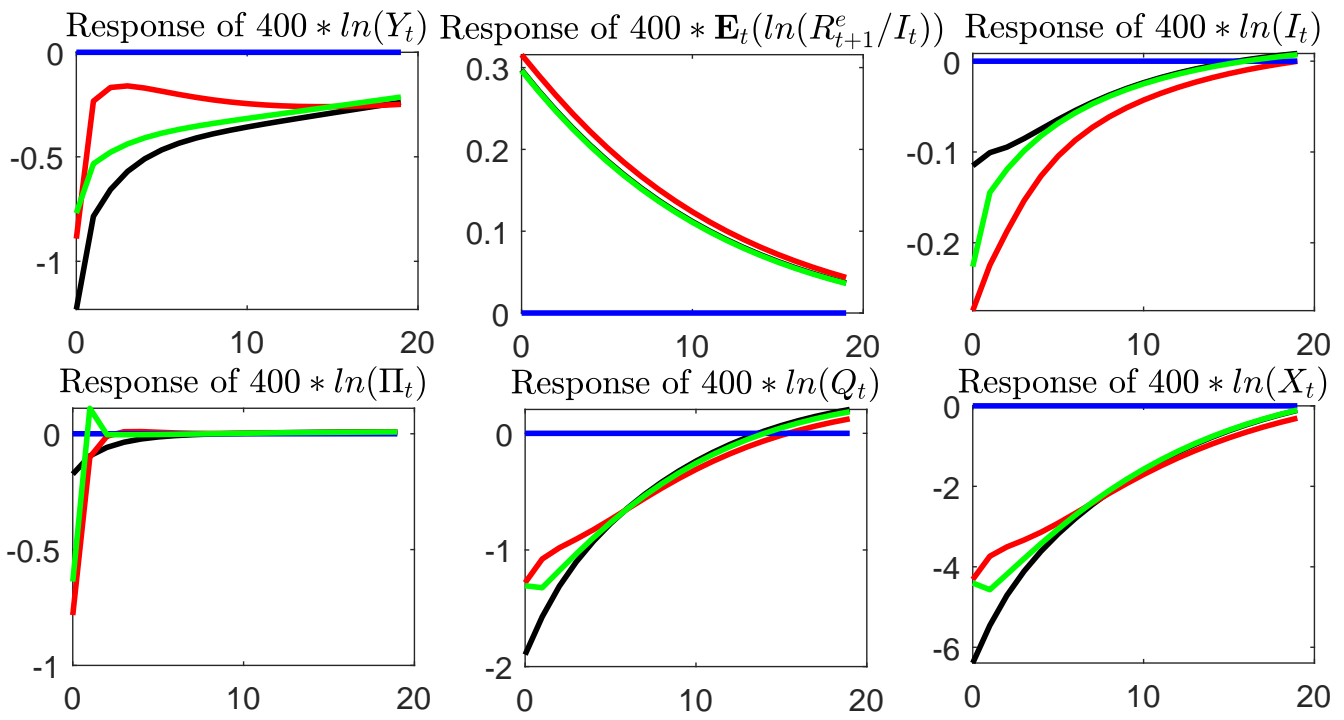

Response of $100 * \Phi\left(\bar{\omega}_{t} \mid \mu_{\omega, t}, \sigma_{\omega, t}\right)$ Response of $100 * N_{t}^{r} / Q_{t} K_{t+1}$

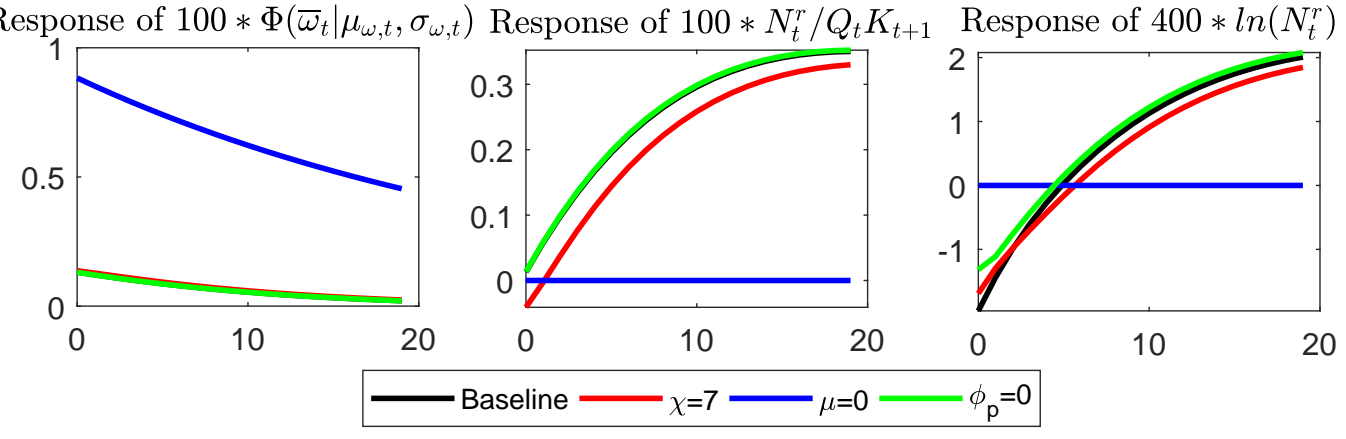


Figure 7D. Generalized Impulse Response Functions: Response to a +1 Standard Deviation Interest Rate Rule Shock, Various Model Specifications, Conditional on Mid-Values of the Endogenous Spread.
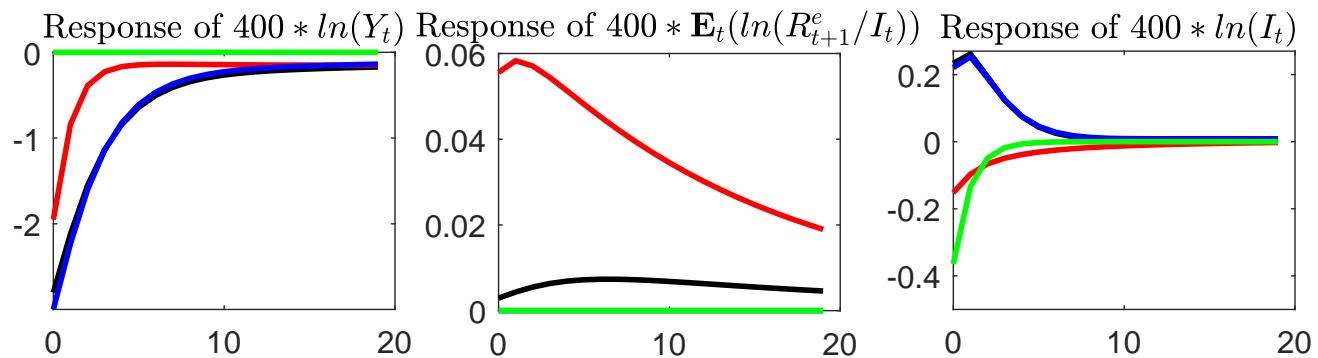

Response of $400 * \ln \left(\Pi_{t}\right)$
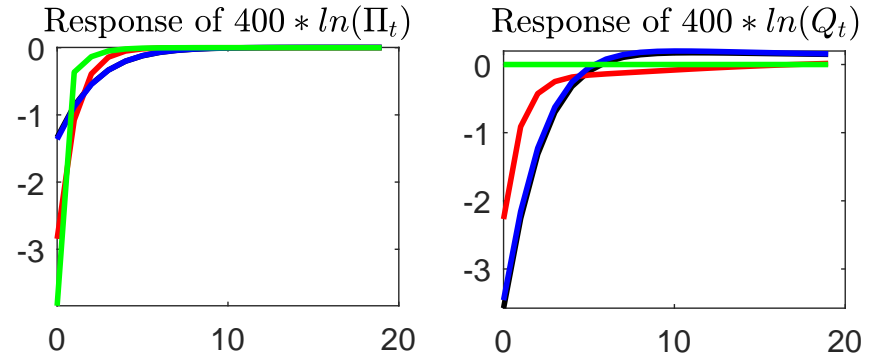

Response of $400 * \ln \left(X_{t}\right)$
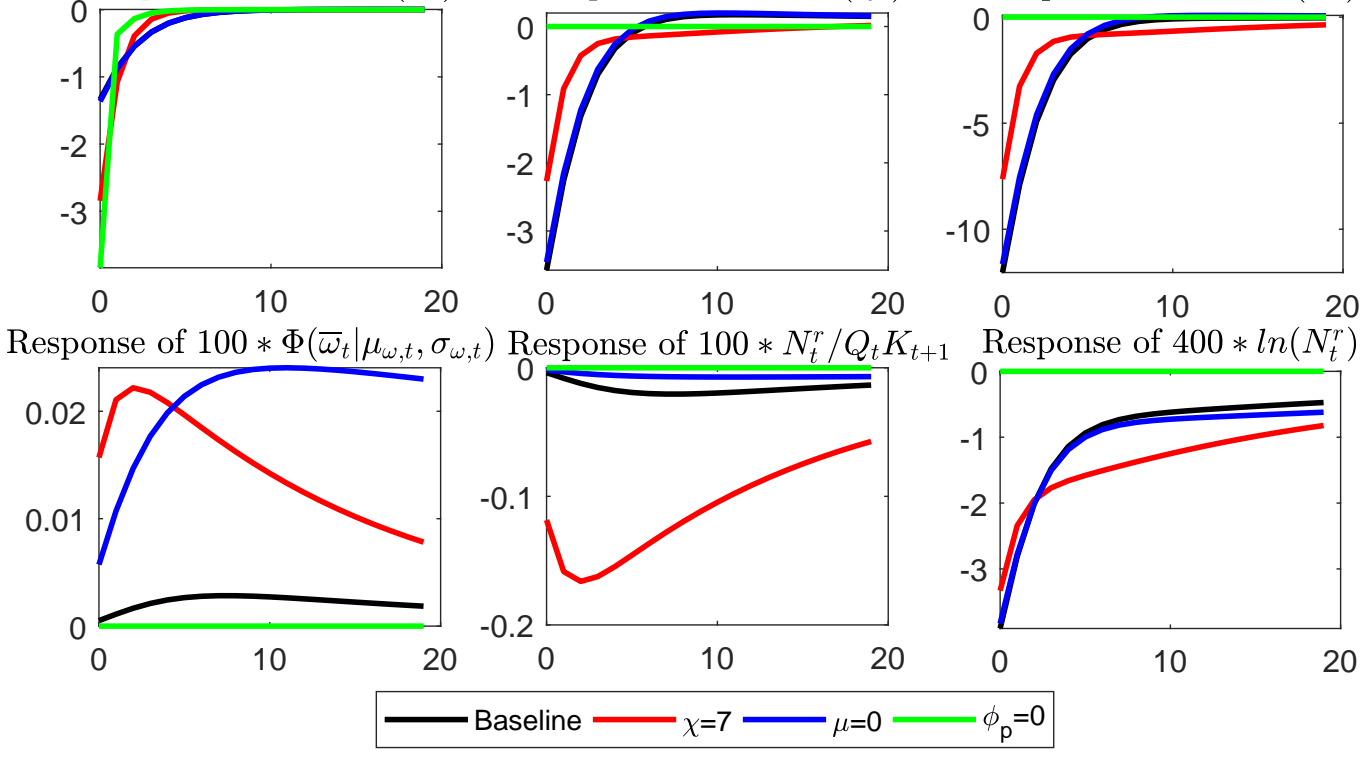
Figure 7E. Generalized Impulse Response Functions: Response to a +1 Standard Deviation Interest Rate Stochastic Volatility Shock, Various Model Specifications, Conditional on Mid-Values of the Endogenous Spread.

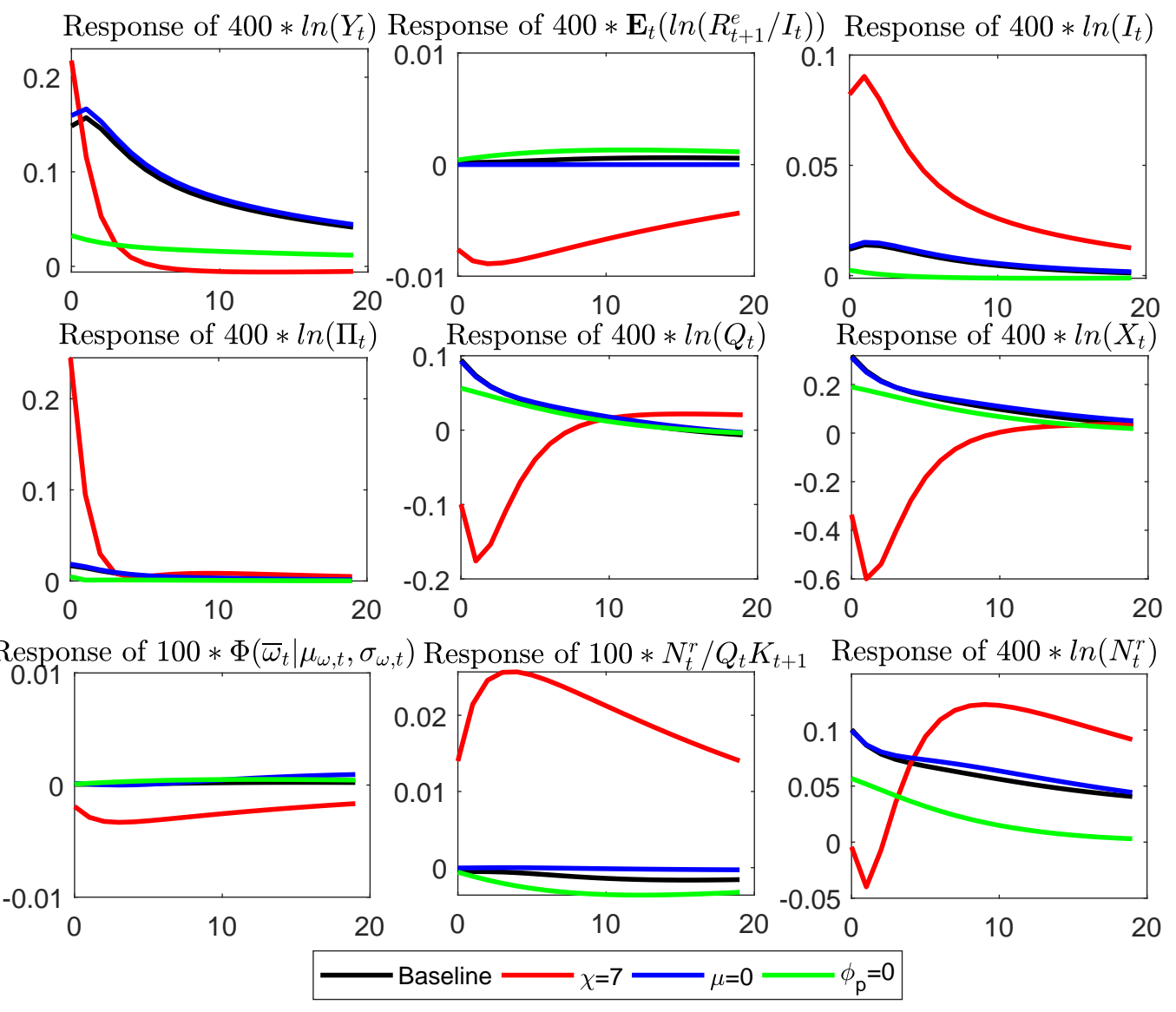


Figure 8A. Generalized Impulse Response Functions: Response to a +1 Standard Deviation TFP Shock, Various Model Specifications, Conditional on High-Values of the Endogenous Spread.
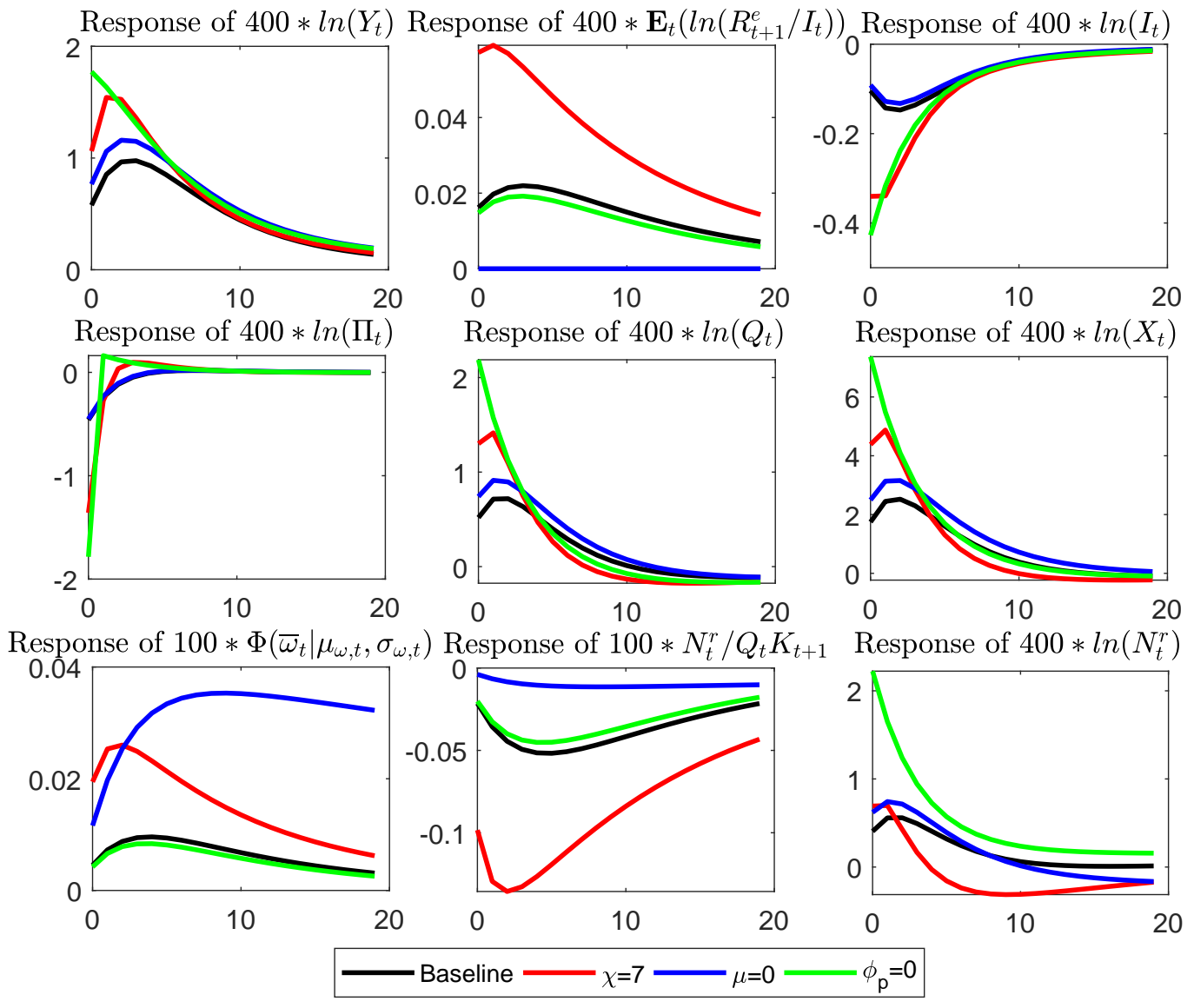
Figure 8B. Generalized Impulse Response Functions: Response to a +1 Standard Deviation TFP Stochastic VolatilityShock, Various Model Specifications, Conditional on Hih-Values of the Endogenous Spread.
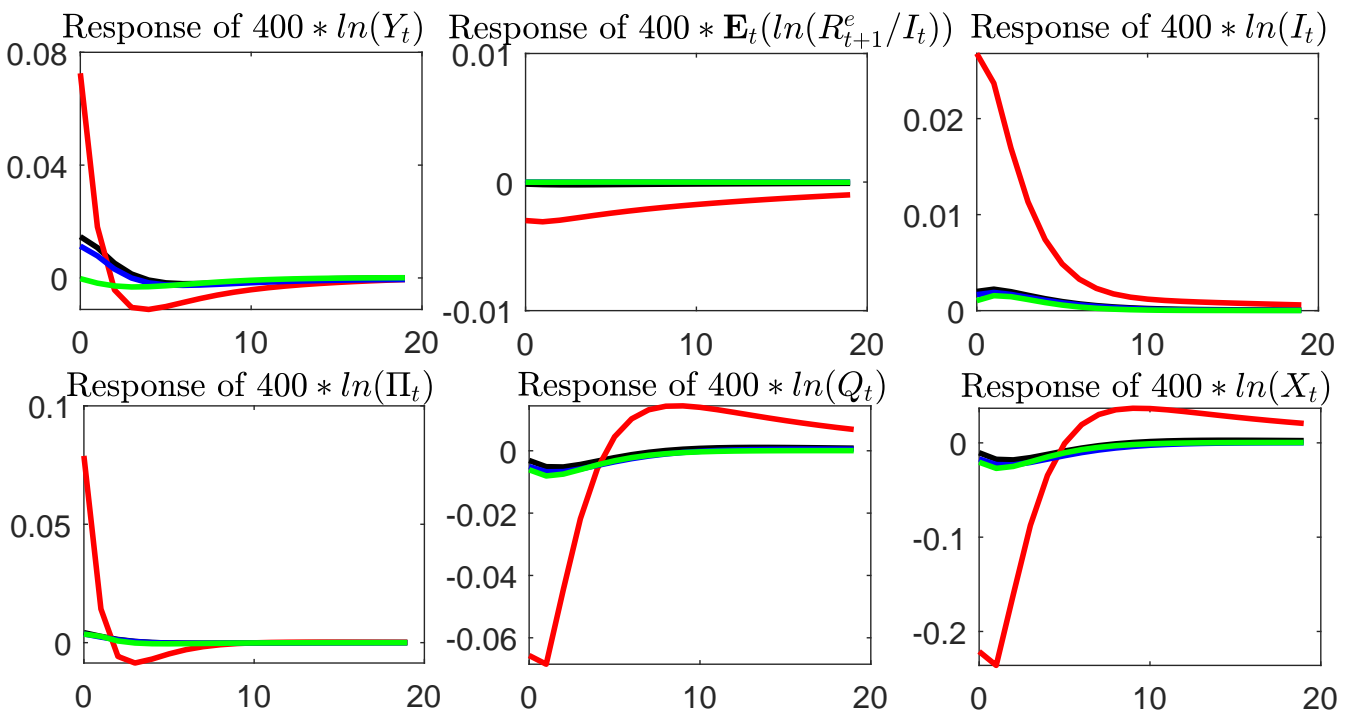

Response of $100 * \Phi\left(\bar{\omega}_{t} \mid \mu_{\omega, t}, \sigma_{\omega, t}\right)$ Response of $100 * N_{t}^{r} / Q_{t} K_{t+1}$ Response of $400 * \ln \left(N_{t}^{r}\right)$
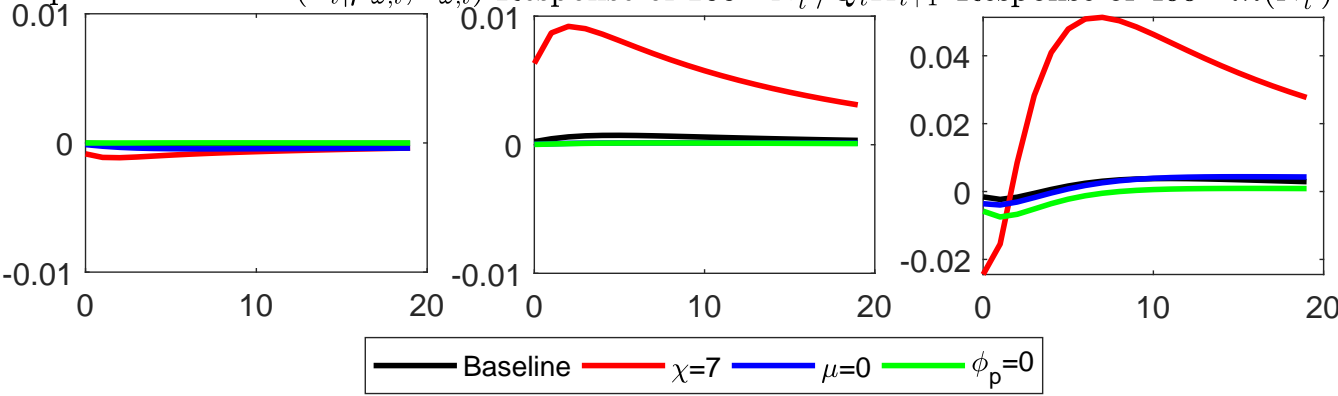
Figure 8C. Generalized Impulse Response Functions: Response to a $+\mathbf{1}$ Standard Deviation Micro-Uncertainty Shock, Various Model Specifications, Conditional on High-Values of the Endogenous Spread.
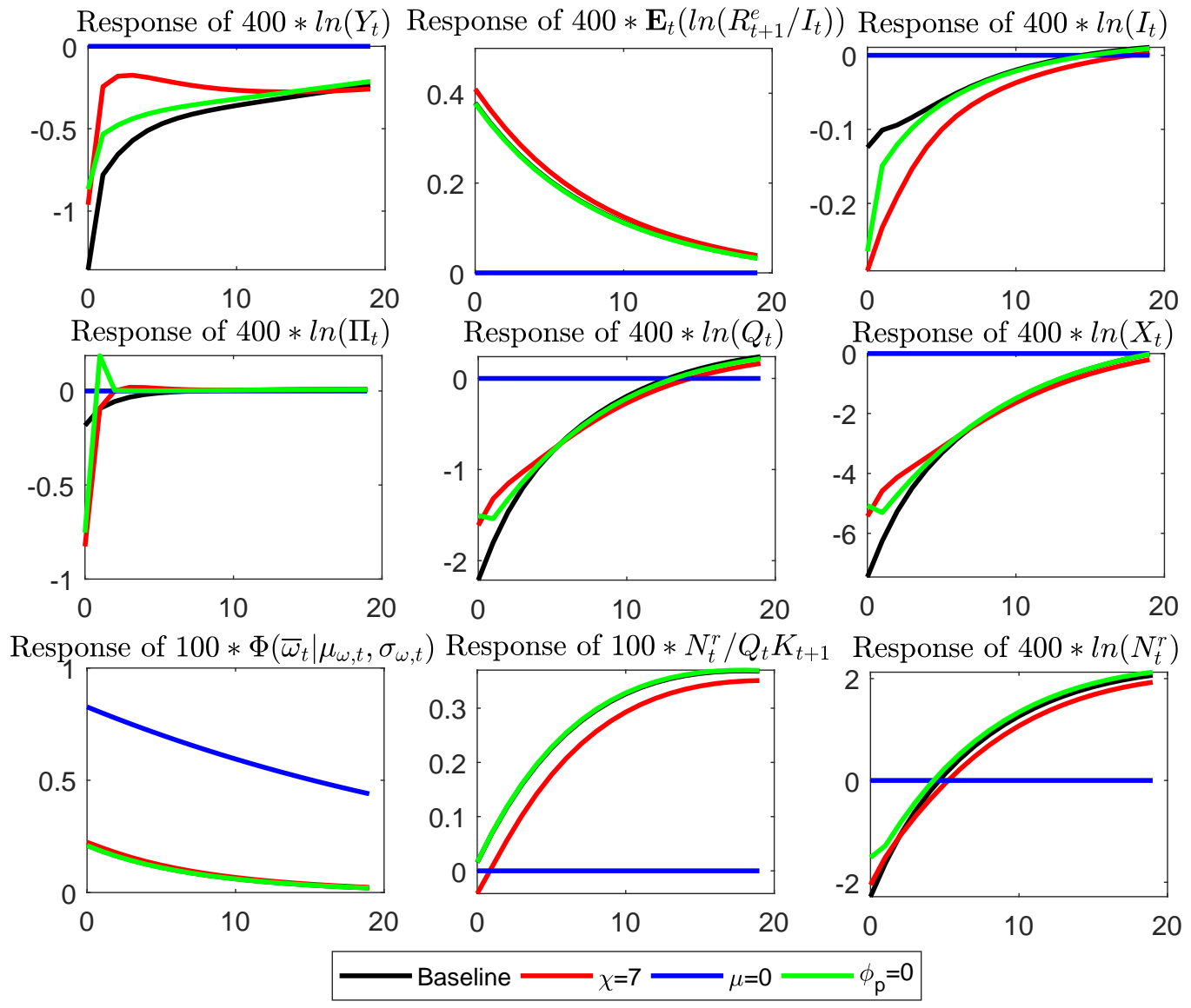
Figure 8D. Generalized Impulse Response Functions: Response to a +1 Standard Deviation Interest Rate Rule Shock, Various Model Specifications, Conditional on High-Values of the Endogenous Spread.
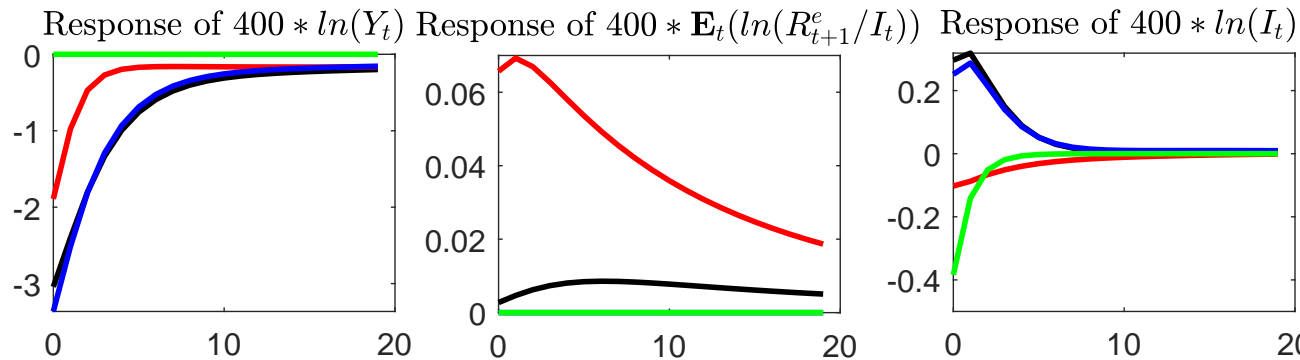

Response of $400 * \ln \left(\Pi_{t}\right)$

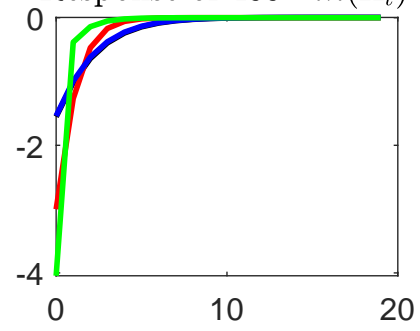

Response of $400 * \ln \left(Q_{t}\right)$
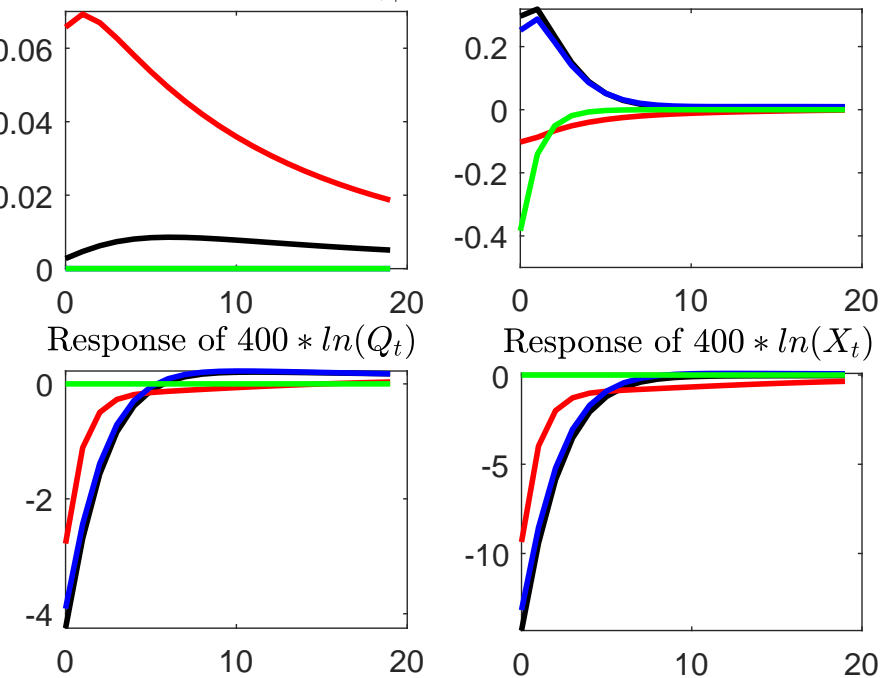

Response of $400 * \ln \left(X_{t}\right)$

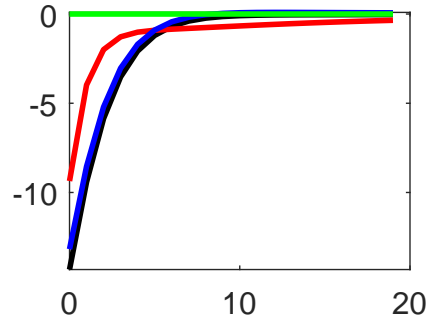

Response of $100 * \Phi\left(\bar{\omega}_{t} \mid \mu_{\omega, t}, \sigma_{\omega, t}\right.$
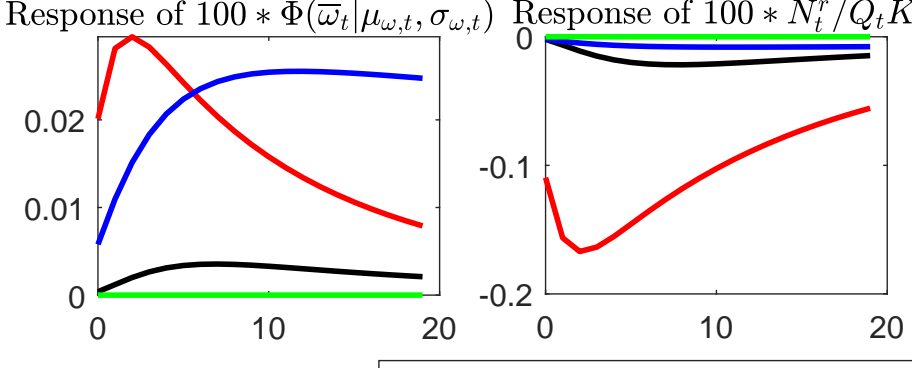

Response of $400 * \ln \left(N_{t}^{r}\right)$

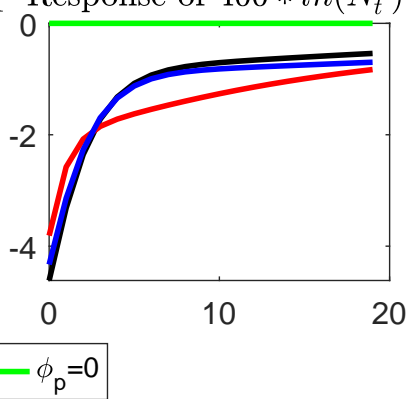


Figure 8E. Generalized Impulse Response Functions: Response to a +1 Standard Deviation Interest Rate Stochastic Volatility Shock, Various Model Specifications, Conditional on High-Values of the Endogenous Spread.
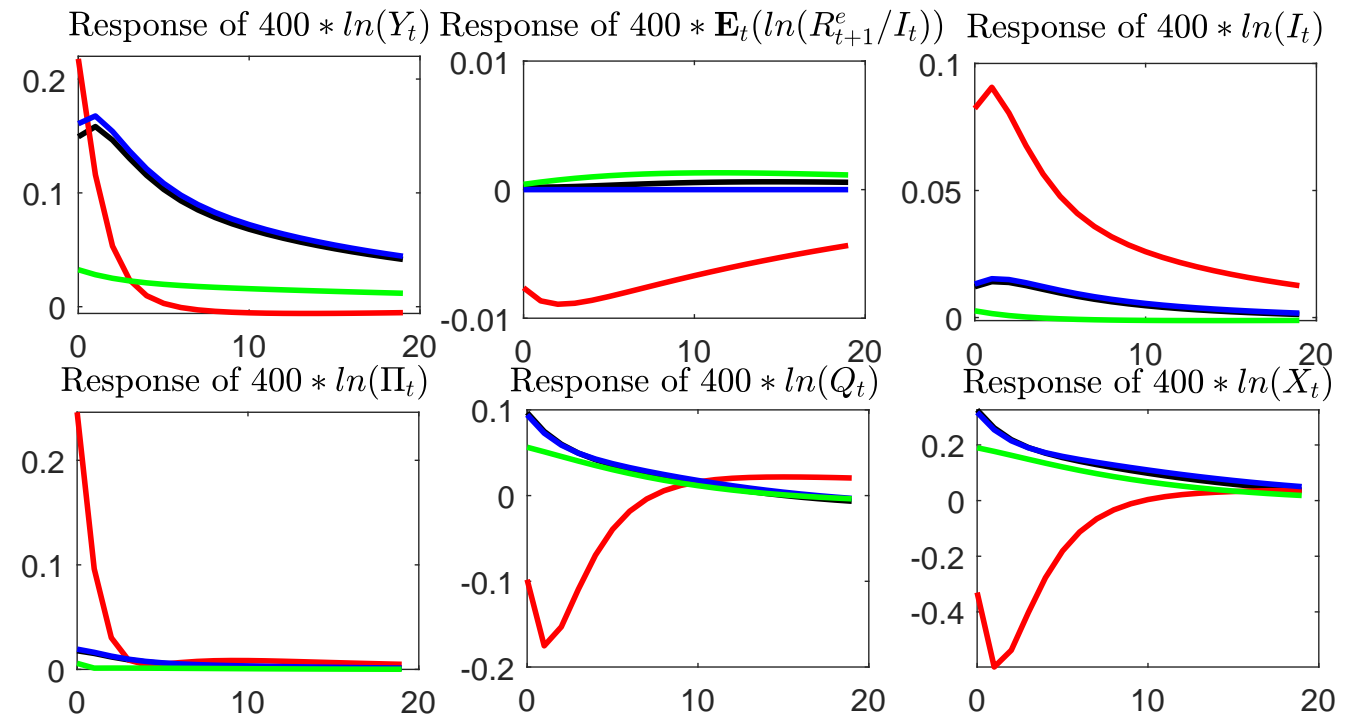

Response of $100 * \Phi\left(\bar{\omega}_{t} \mid \mu_{\omega, t}, \sigma_{\omega, t}\right)$ Response of $100 * N_{t}^{r} / Q_{t} K_{t+1}$ Response of $400 * \ln \left(N_{t}^{r}\right)$

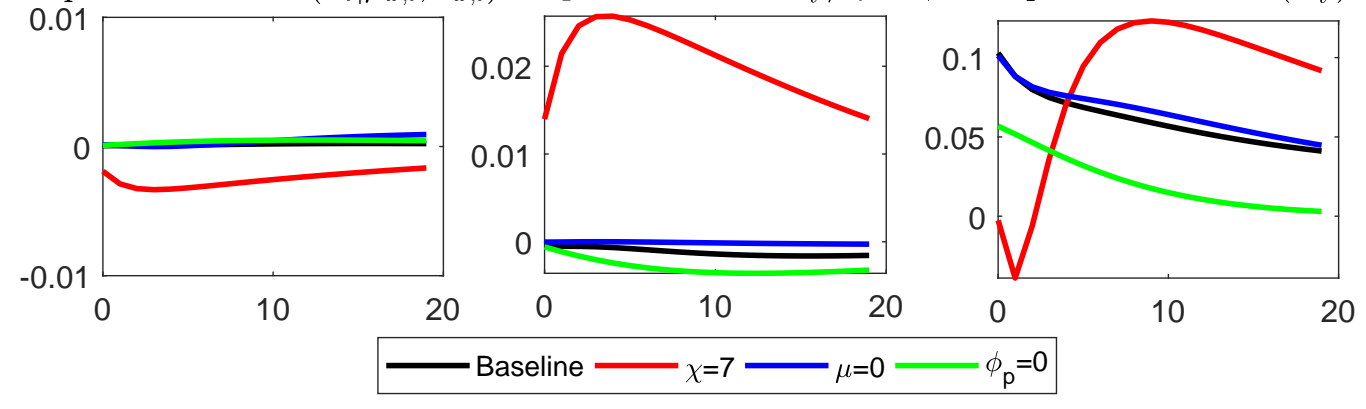


Figure 9A. Generalized Impulse Response Functions: Response to a +1 Standard Deviation TFP Shock, Conditional on High-Values of Spread, Interest Rate, and Equity Ratio.
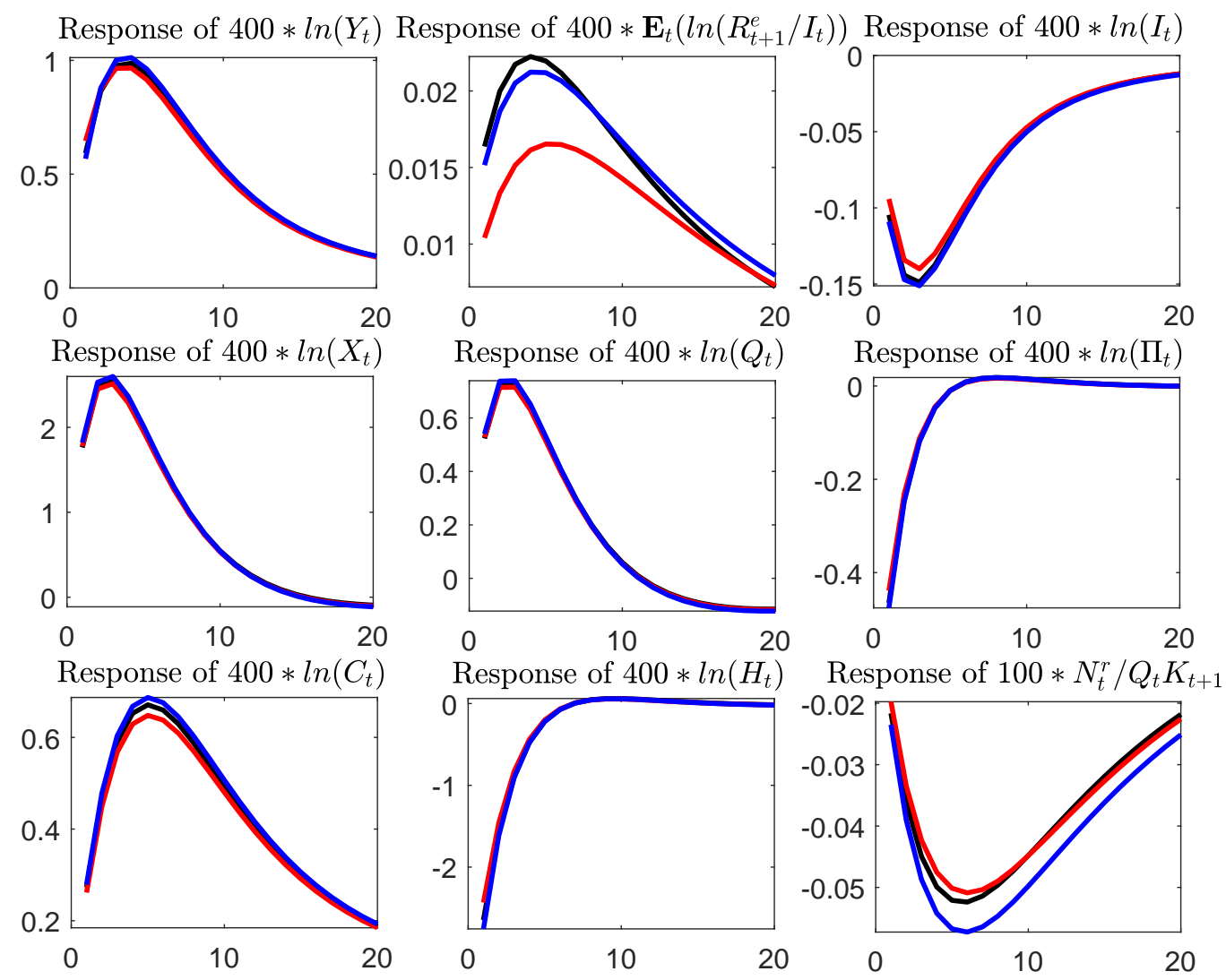

High spread

High interest rate

High equity ratio 
Figure 9B. Generalized Impulse Response Functions: Response to a +1 Standard Deviation TFP Stochastic Volatility Shock, Conditional on High-Values of Spread, Interest Rate, and Equity Ratio.
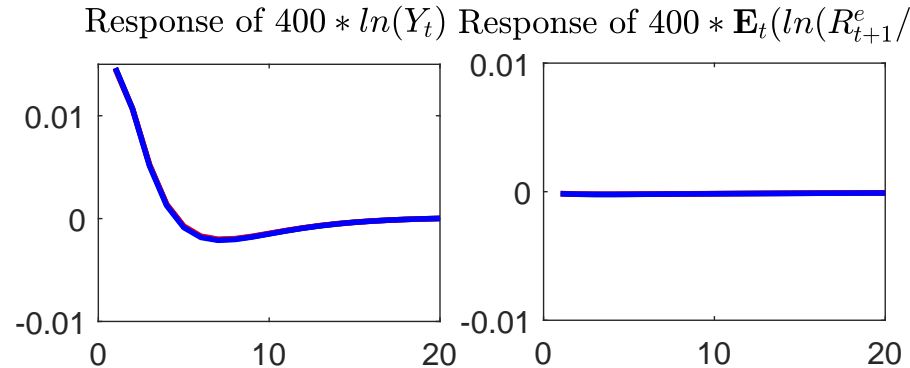

$\left.\left.I_{t}\right)\right)$ Response of $400 * \ln \left(I_{t}\right)$
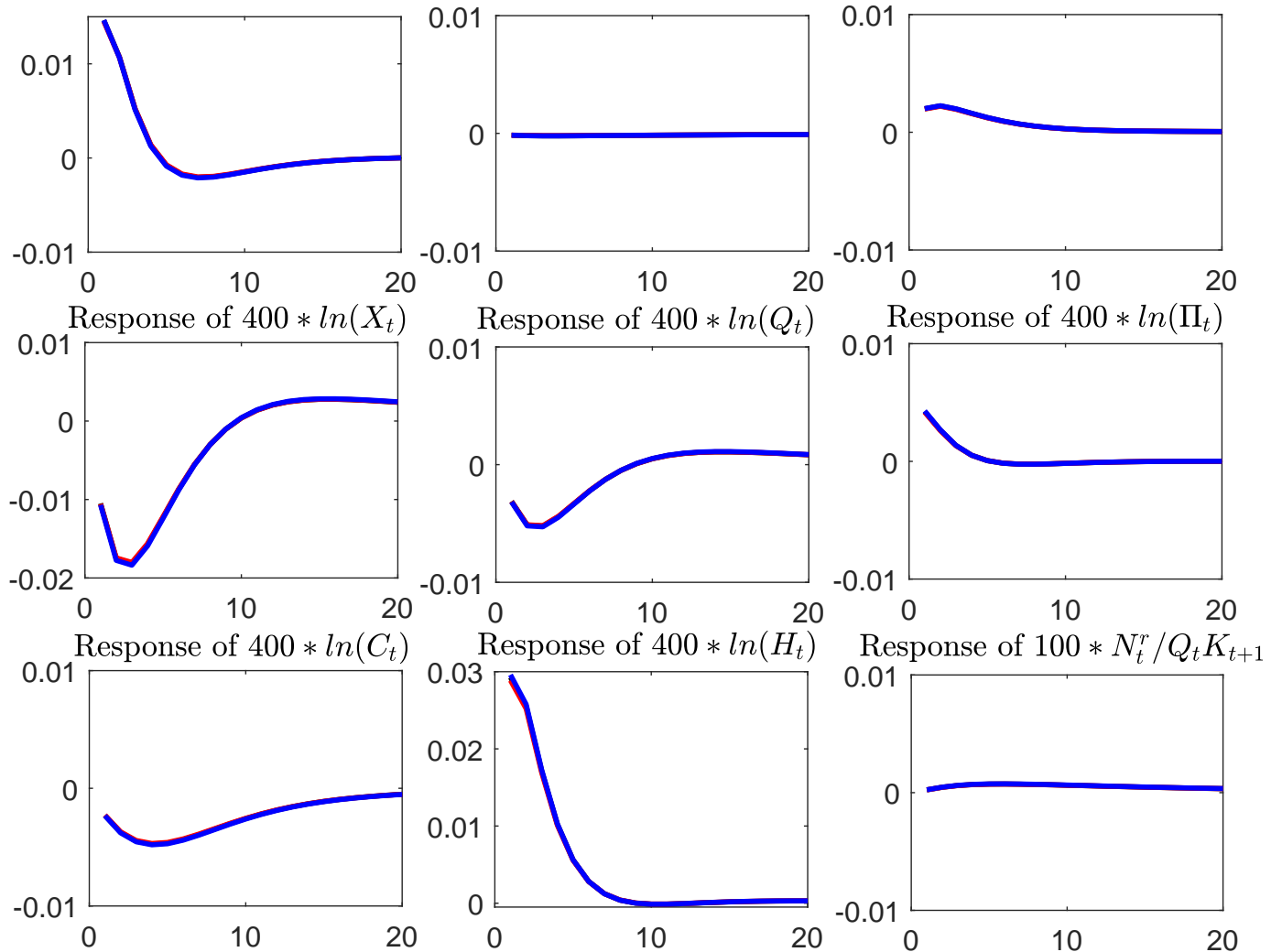

High spread $\longrightarrow$ High interest rate $\longrightarrow$ High equity ratio 
Figure 9C. Generalized Impulse Response Functions: Response to a +1 Standard Deviation Micro-Uncertainty Shock, Conditional on High-Values of Spread, Interest Rate, and Equity Ratio.
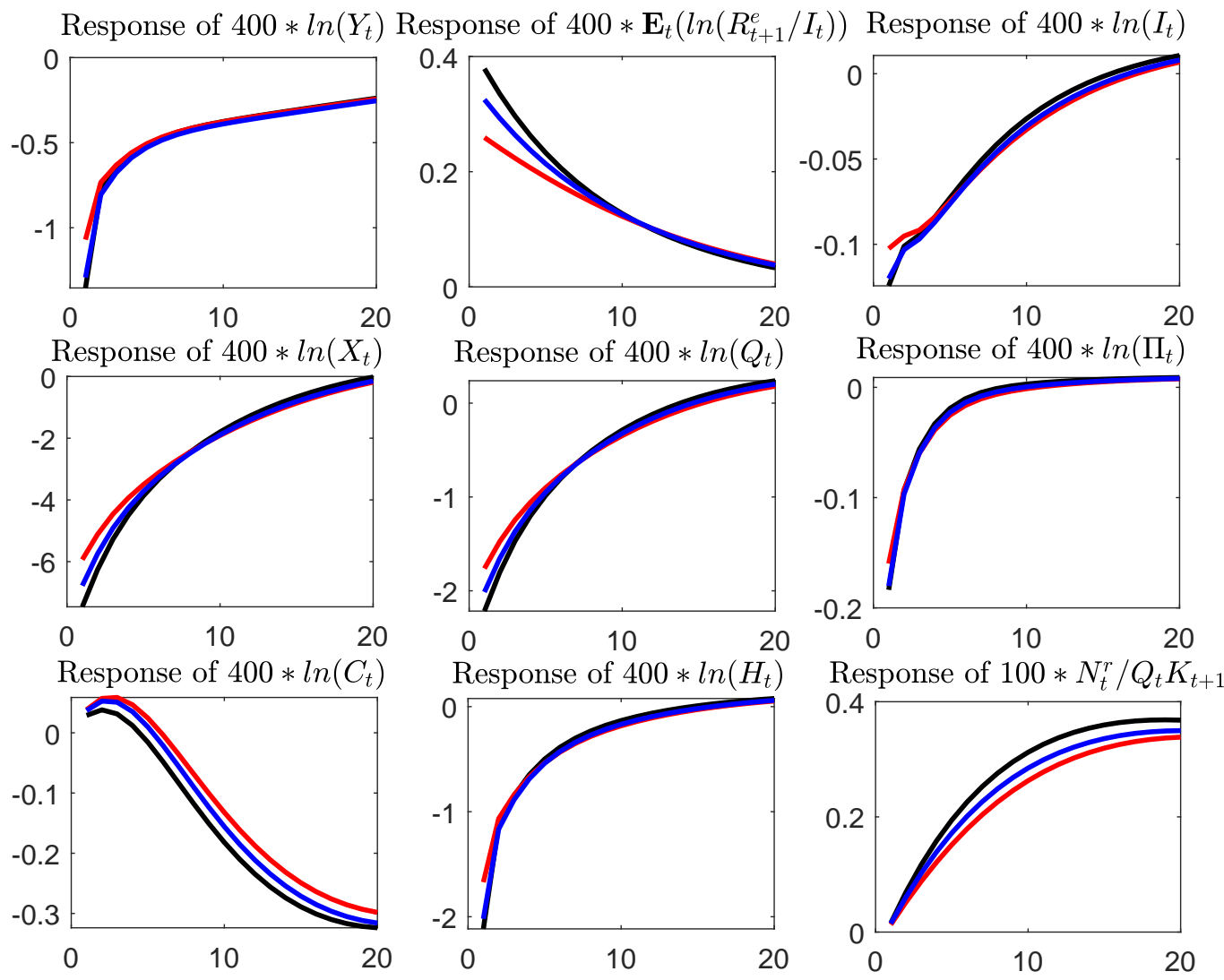

$\longrightarrow$ High spread $\longrightarrow$ High interest rate $\longrightarrow$ High equity ratio 
Figure 9D. Generalized Impulse Response Functions: Response to a +1 Standard Deviation Interest Rate Rule Shock, Conditional on High-Values of Spread, Interest Rate, and Equity Ratio.
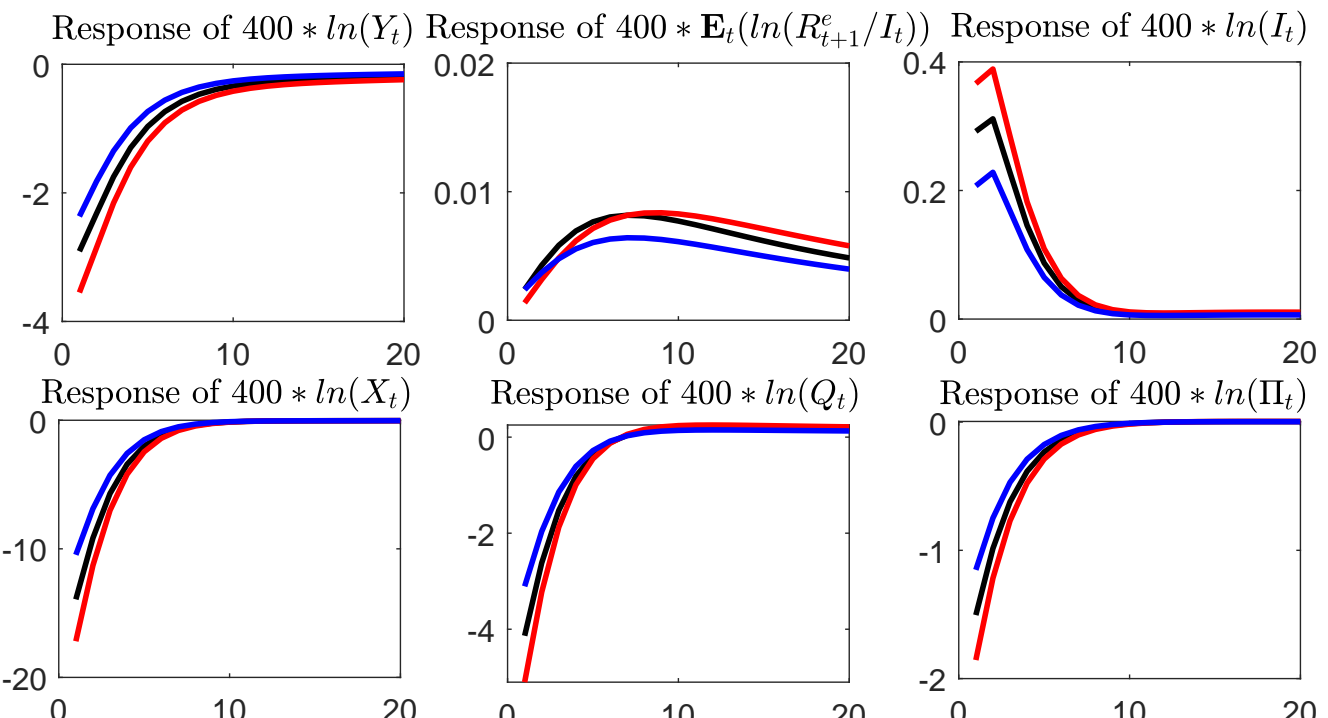

Response of $400 * \ln \left(Q_{t}\right)$

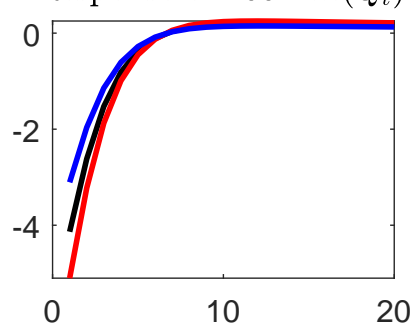

Response of $400 * \ln \left(\Pi_{t}\right)$
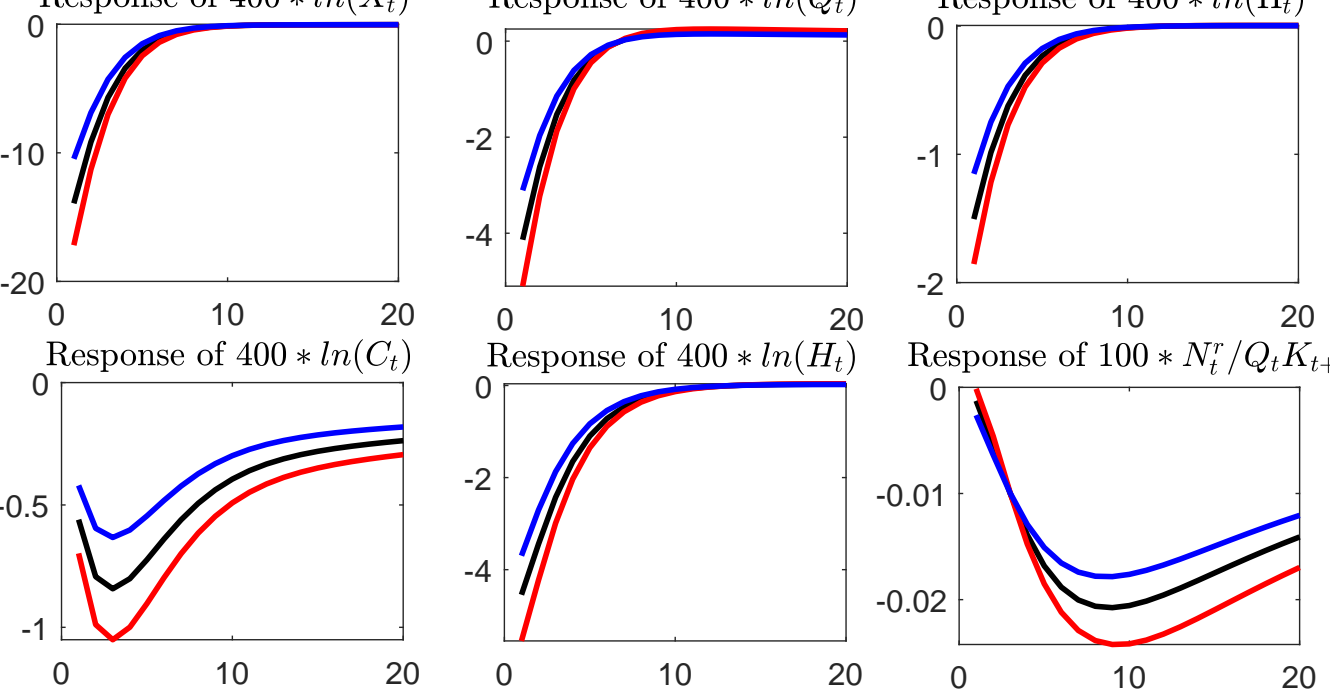

Response of $400 * \ln \left(H_{t}\right)$
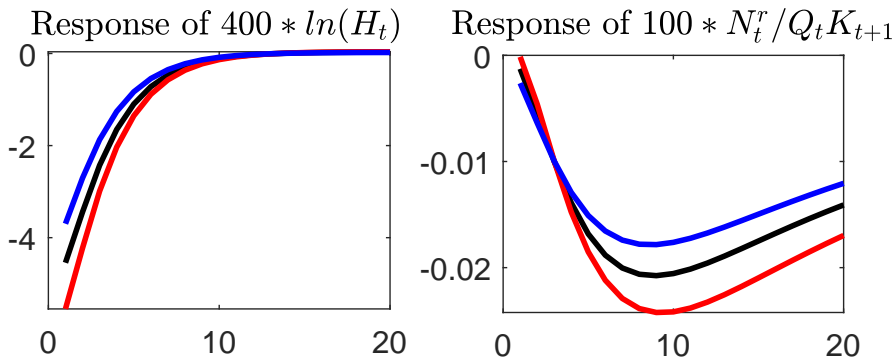

$\longrightarrow$ High spread $\longrightarrow$ High interest rate $\longrightarrow$ High equity ratio 
Figure 9E. Generalized Impulse Response Functions: Response to a +1 Standard Deviation Interest Rate Stochastic Volatility Shock, Conditional on High-Values of Spread, Interest Rate, and Equity Ratio.
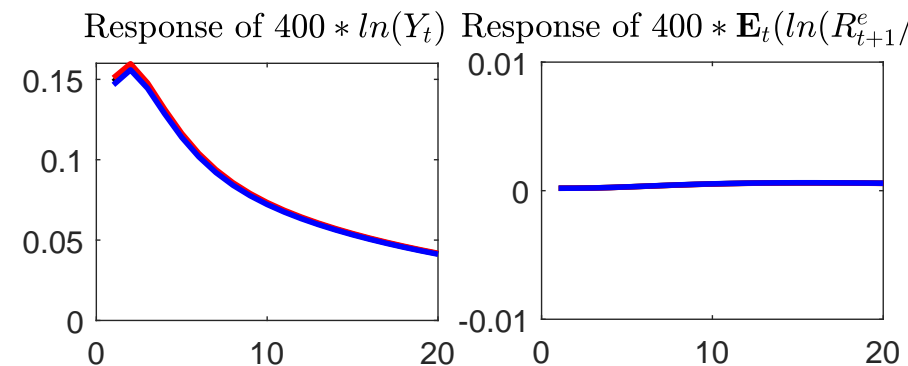

$\left.\left./ I_{t}\right)\right)$ Response of $400 * \ln \left(I_{t}\right)$
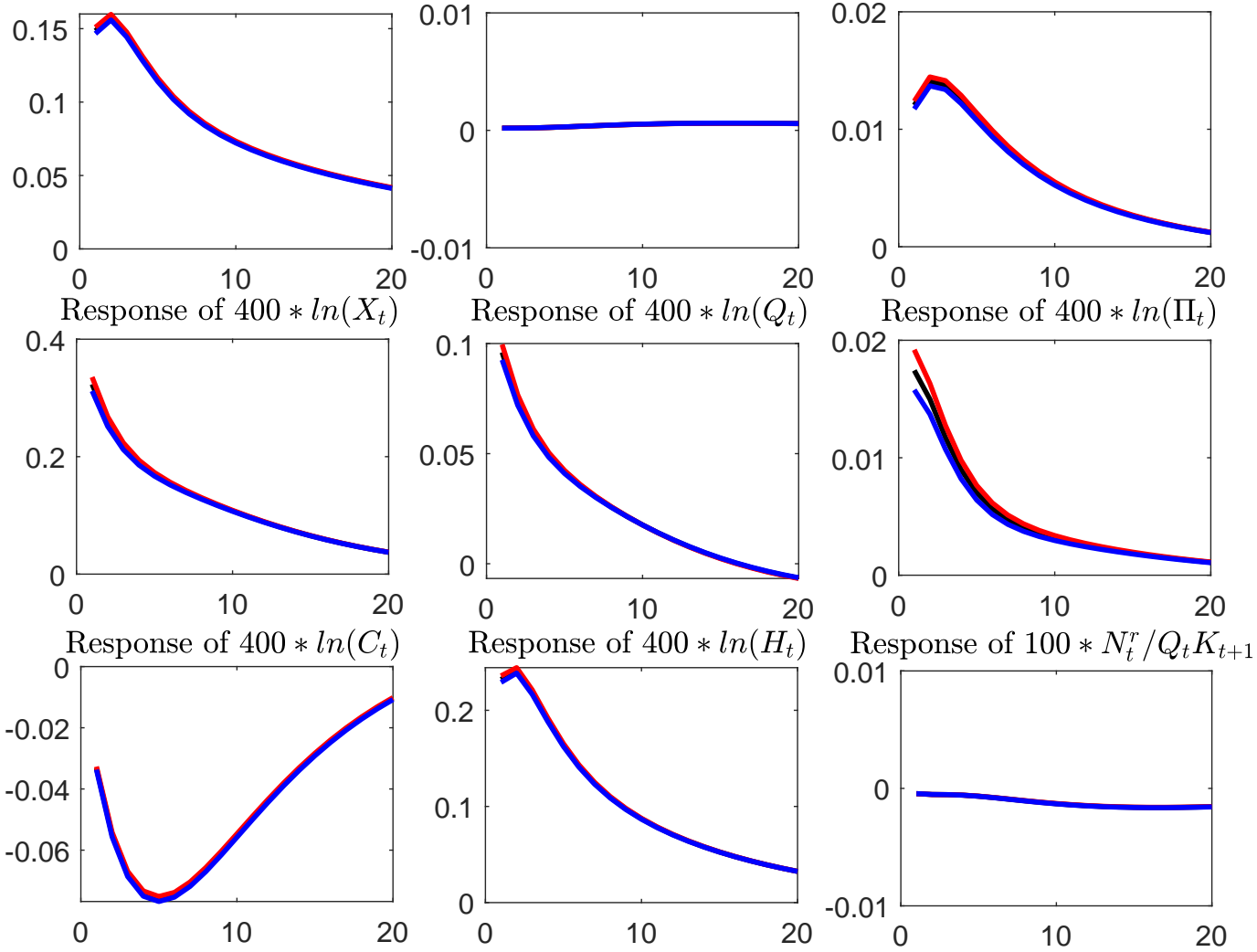

High spread $\longrightarrow$ High interest rate

High equity ratio 
Figure 10A. Generalized Impulse Response Functions: Response to a +1 Standard Deviation TFP Shock, Conditional on High-Values of Spread, TFP Stochastic Volatility, Interest Rate Stochastic Volatility, and Micro-Uncertainty.
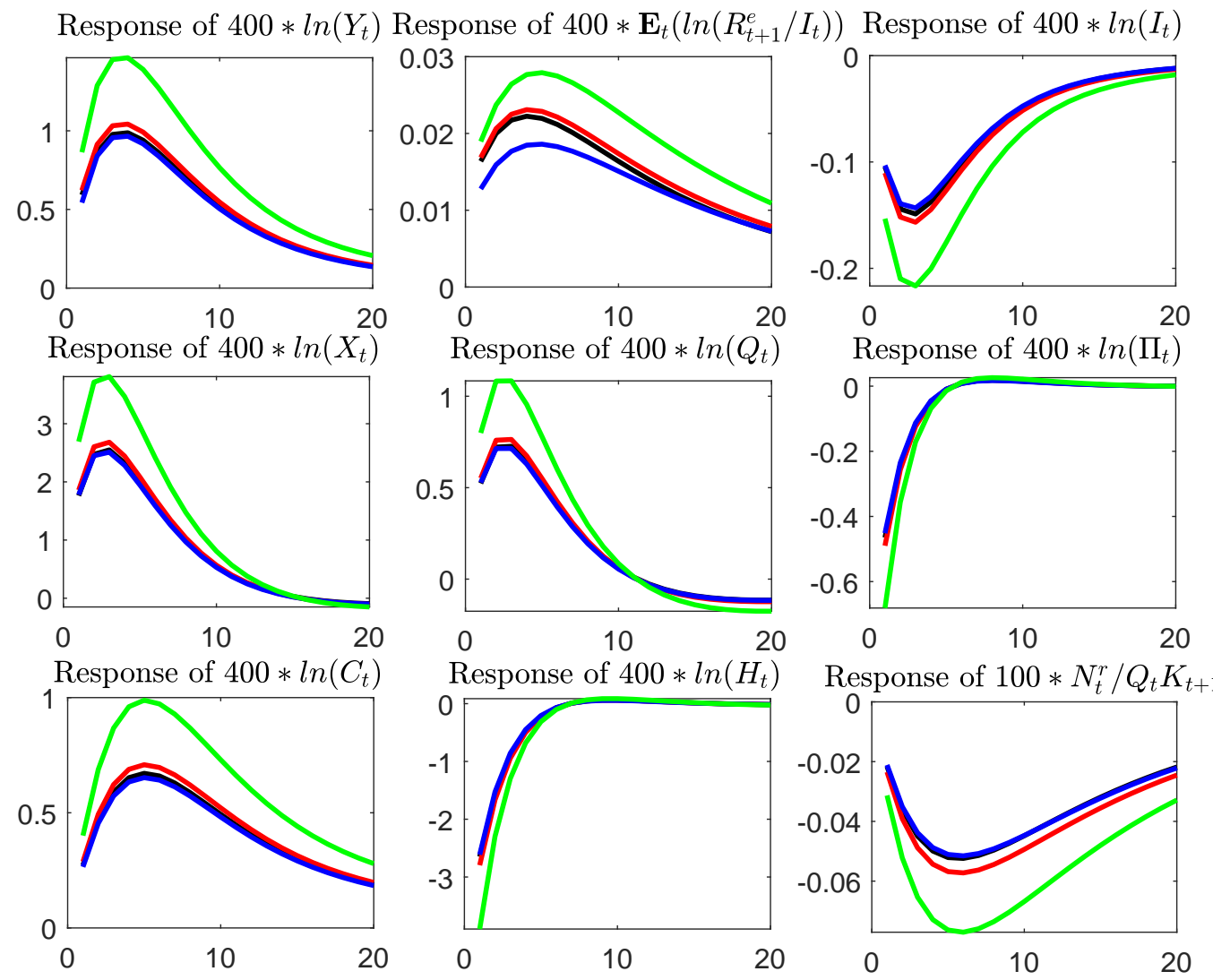

High spread $\longrightarrow$ High micro uncertainty

High policy uncertainty

- High TFP uncertainty 
Figure 10B. Generalized Impulse Response Functions: Response to a +1 Standard Deviation TFP Stochastic Volatility Shock, Conditional on High-Values of Spread, TFP Stochastic Volatility, Interest Rate Stochastic Volatility, and Micro-Uncertainty.

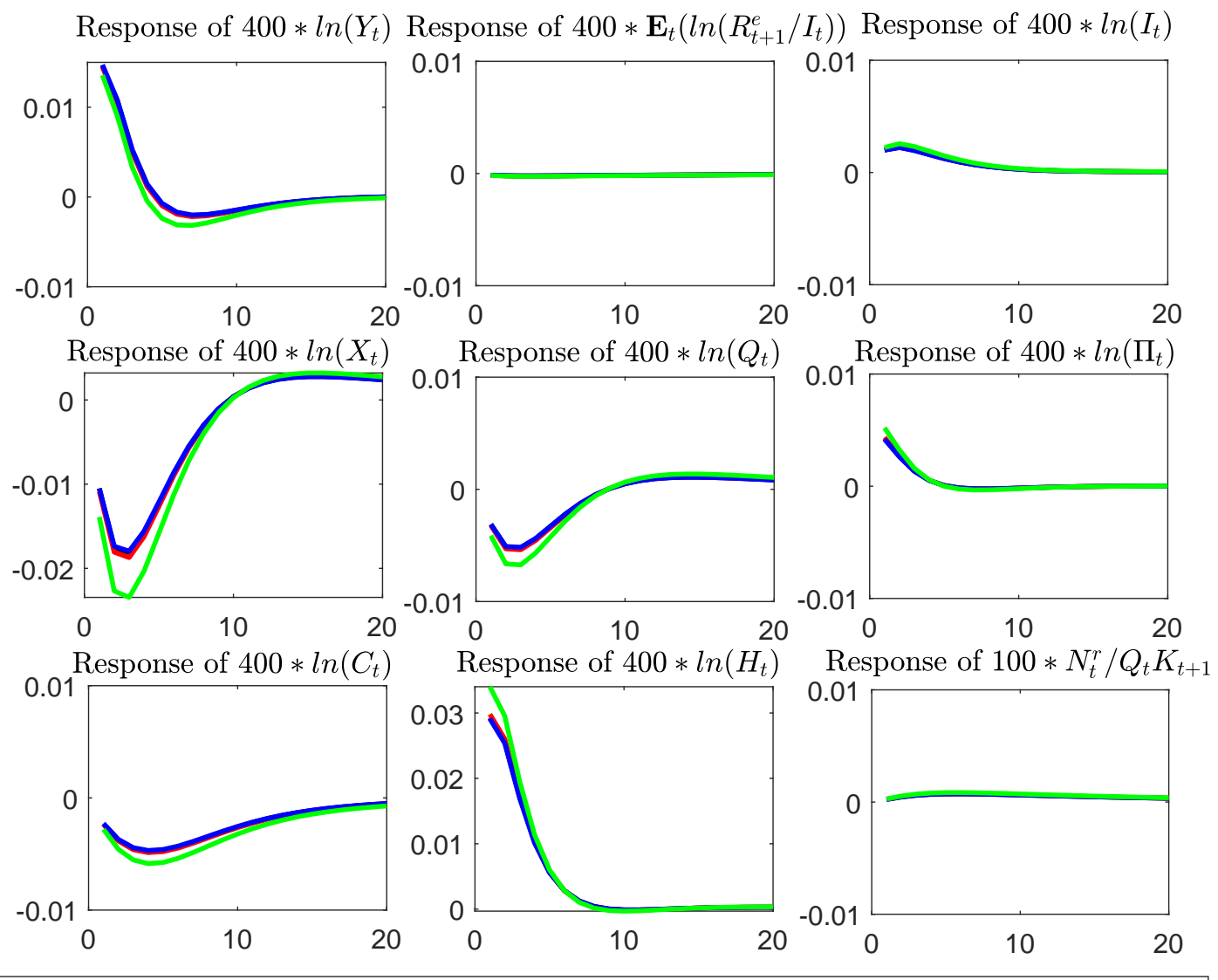

High spread $\longrightarrow$ High micro uncertainty

High policy uncertainty

-High TFP uncertainty 
Figure 10C. Generalized Impulse Response Functions: Response to a +1 Standard Deviation Micro-Uncertainty Shock, Conditional on High-Values of Spread, TFP Stochastic Volatility, Interest Rate Stochastic Volatility, and Micro-Uncertainty.
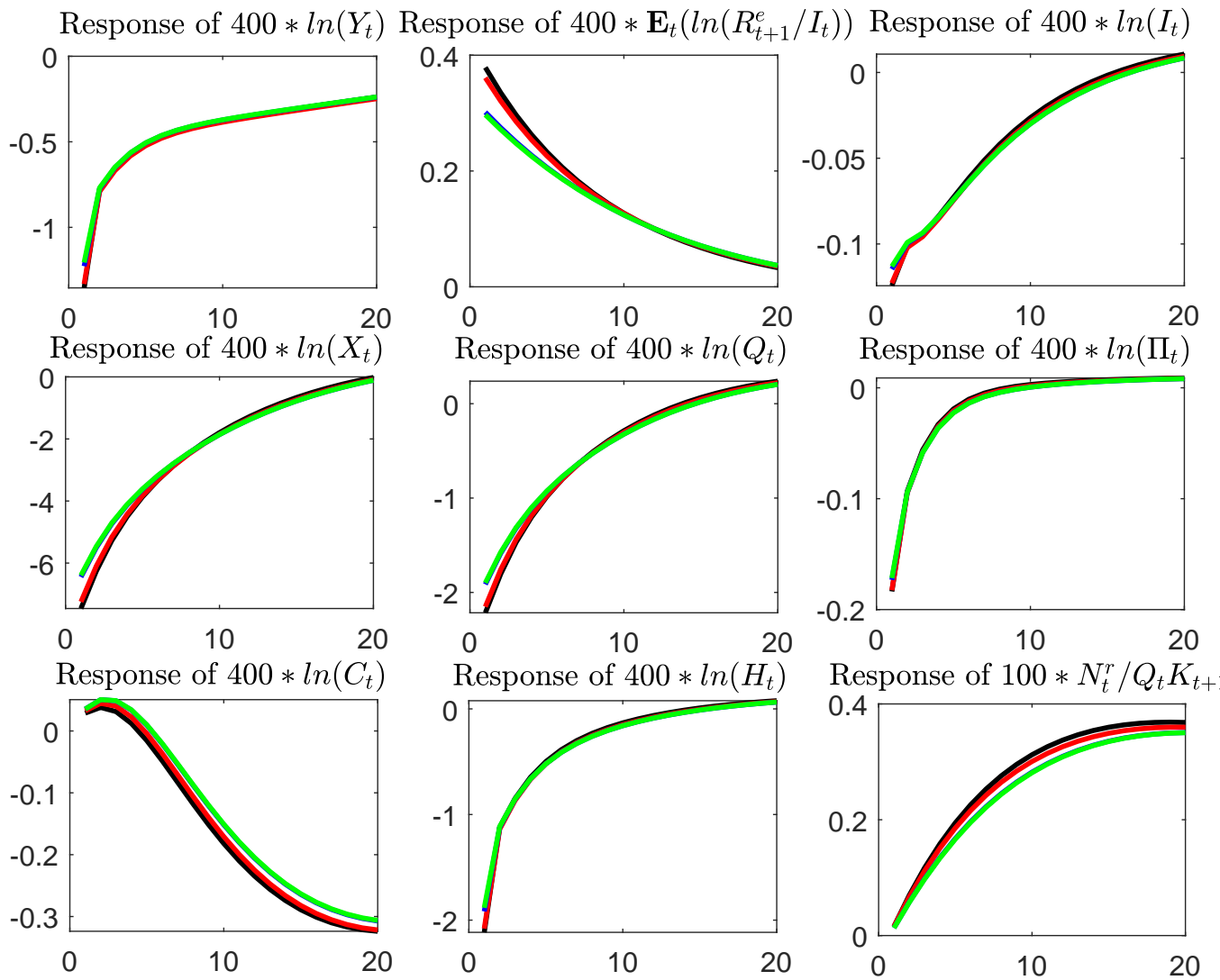
Figure 10D. Generalized Impulse Response Functions: Response to a +1 Standard Deviation Interest Rate Rule Shock, Conditional on High-Values of Spread, TFP Stochastic Volatility, Interest Rate Stochastic Volatility, and Micro-Uncertainty.
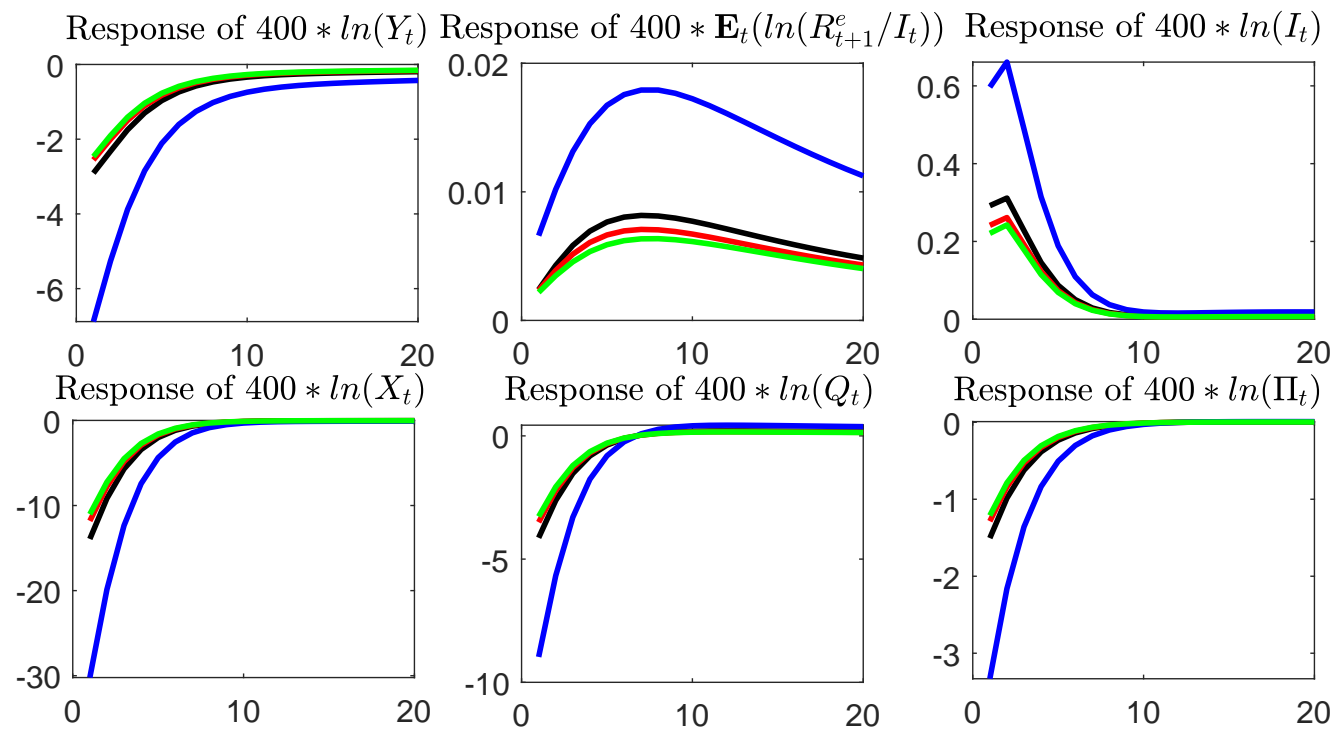

Response of $400 * \ln \left(C_{t}\right)$
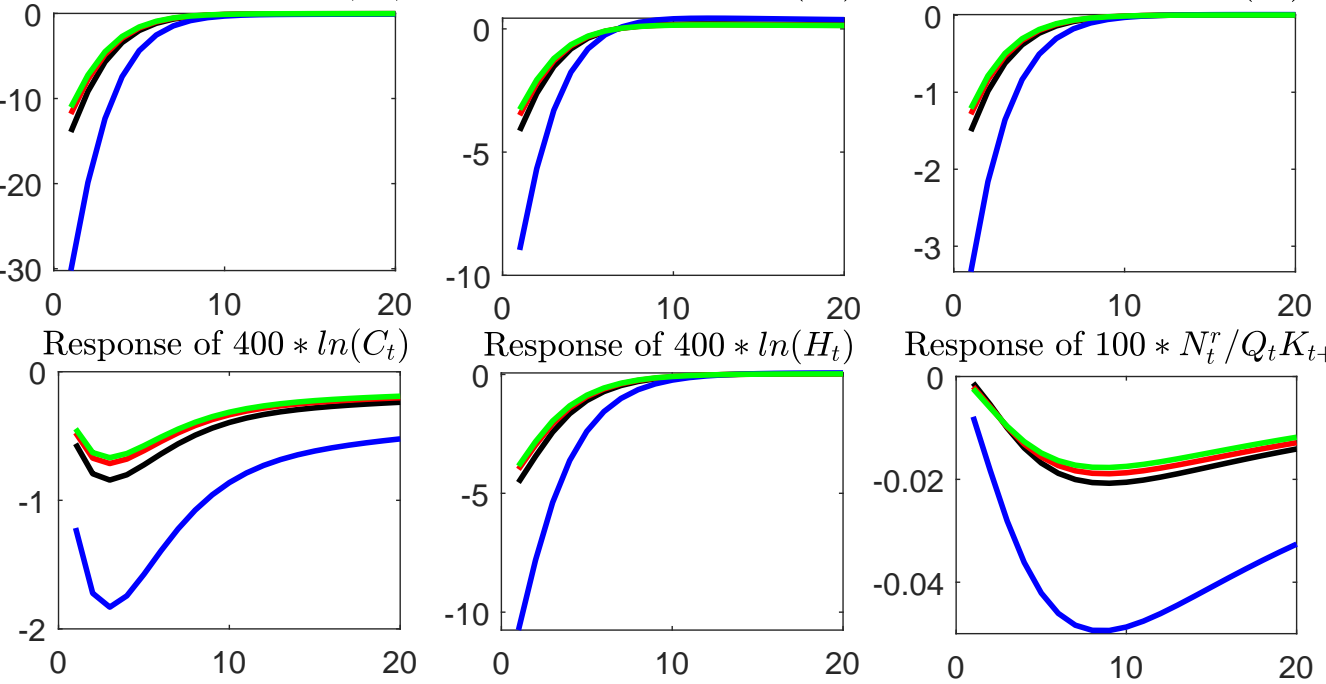

Response of $100 * N_{t}^{r} / Q_{t} K_{t+1}$

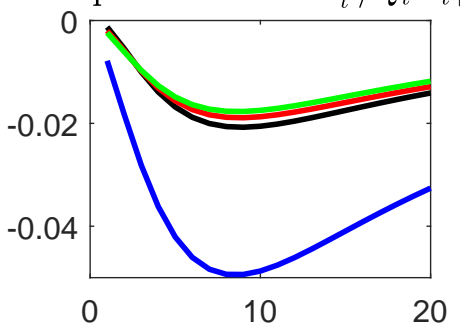

High spread

High micro uncertainty

High policy uncertainty

- High TFP uncertainty 
Figure 10E. Generalized Impulse Response Functions: Response to a +1 Standard Deviation Interest Rate Stochastic Volatility Shock, Conditional on High-Values of Spread, TFP Stochastic Volatility, Interest Rate Stochastic Volatility, and Micro-Uncertainty.
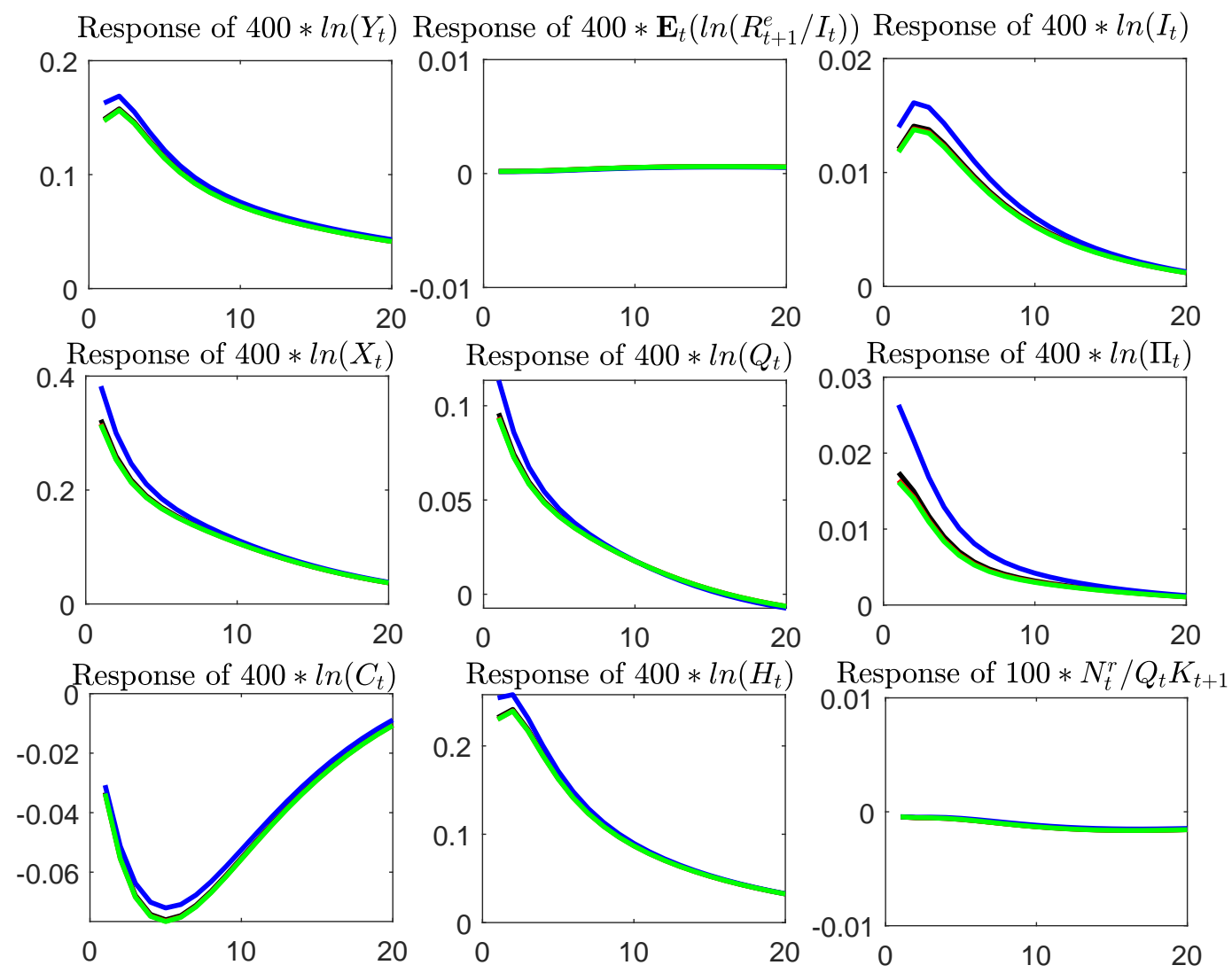
Figure 11. Probability Density Conditional on the Endogenous Spread: Unconditional, Conditional (5th and 95th percentile of Conditioning Variable). Density Smoothed by Epanechnikov Kernel.
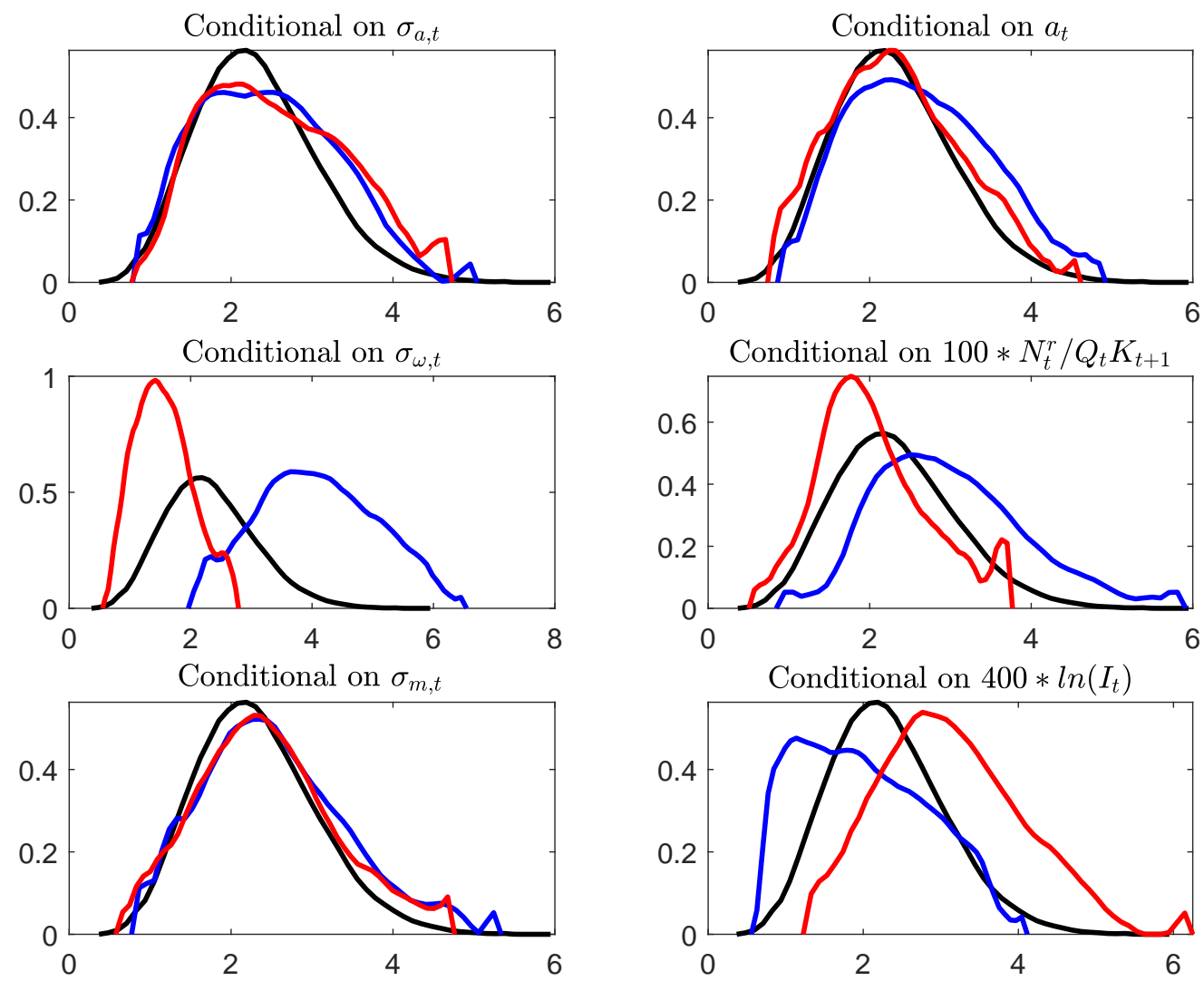

Unconditional $\longrightarrow$ Conditional (95\%)

Conditional (5\%) 
Figure 12A. Joint Distribution of the Endogenous Spread and TFP.

Joint distribution of $400 * \mathbf{E}_{t}\left(\ln \left(R_{t+1}^{e} / I_{t}\right)\right)$ and $a_{t}$
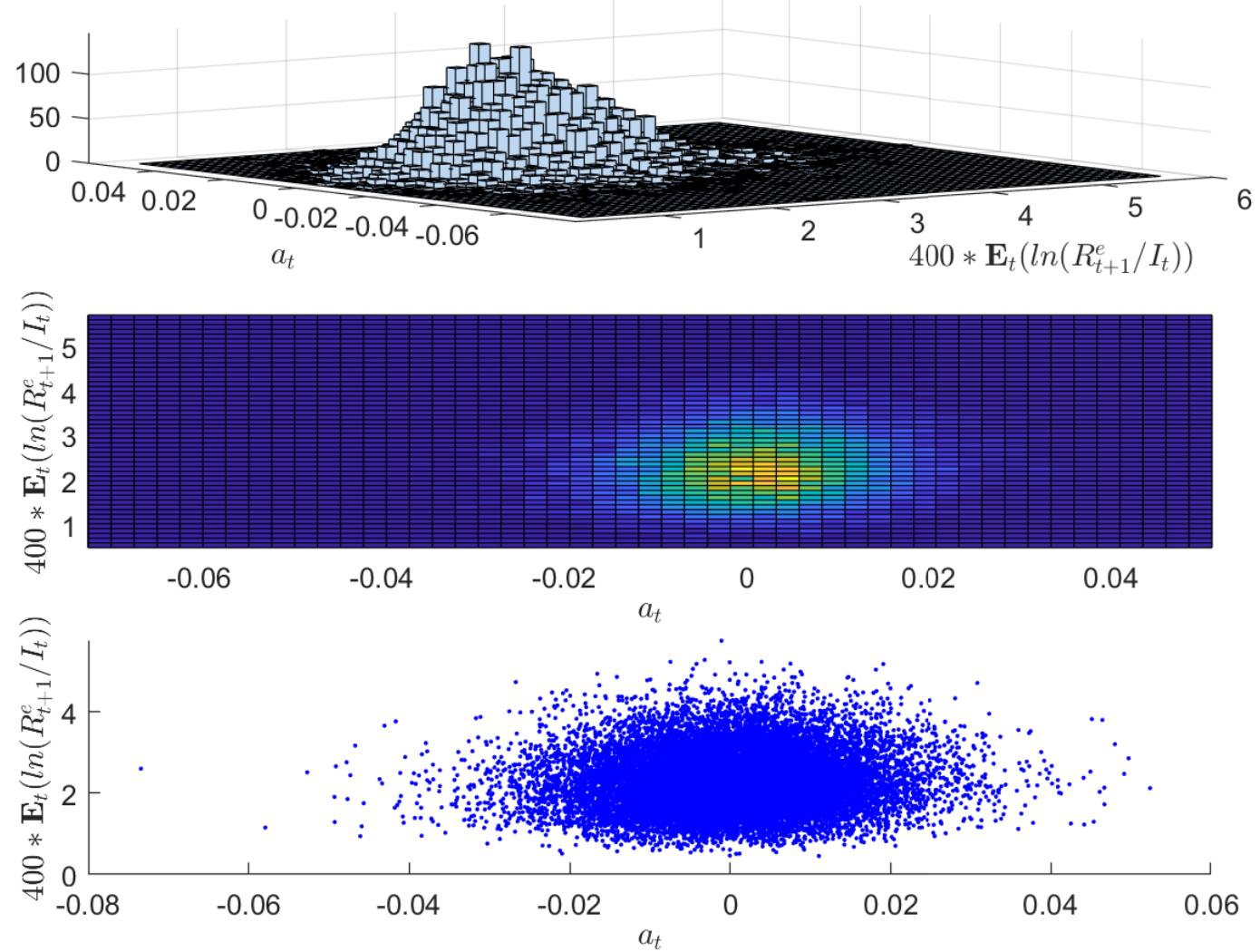
Figure 12B. Joint Distribution of the Endogenous Spread and Interest Rate.
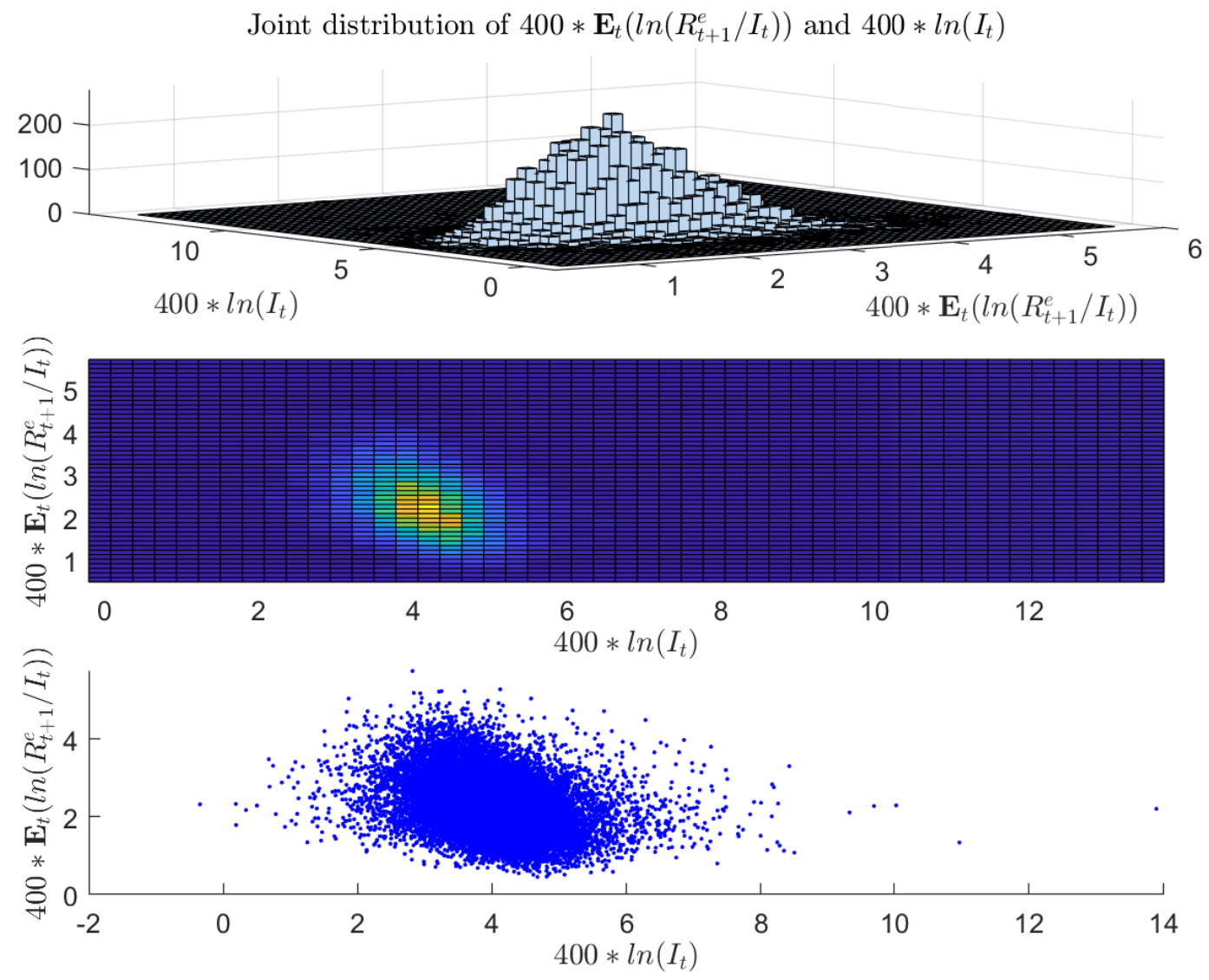
Figure 12C. Joint Distribution of the Endogenous Spread and TFP Stochastic Volatility.

Joint distribution of $400 * \mathbf{E}_{t}\left(\ln \left(R_{t+1}^{e} / I_{t}\right)\right)$ and $\hat{\sigma}_{a, t}$
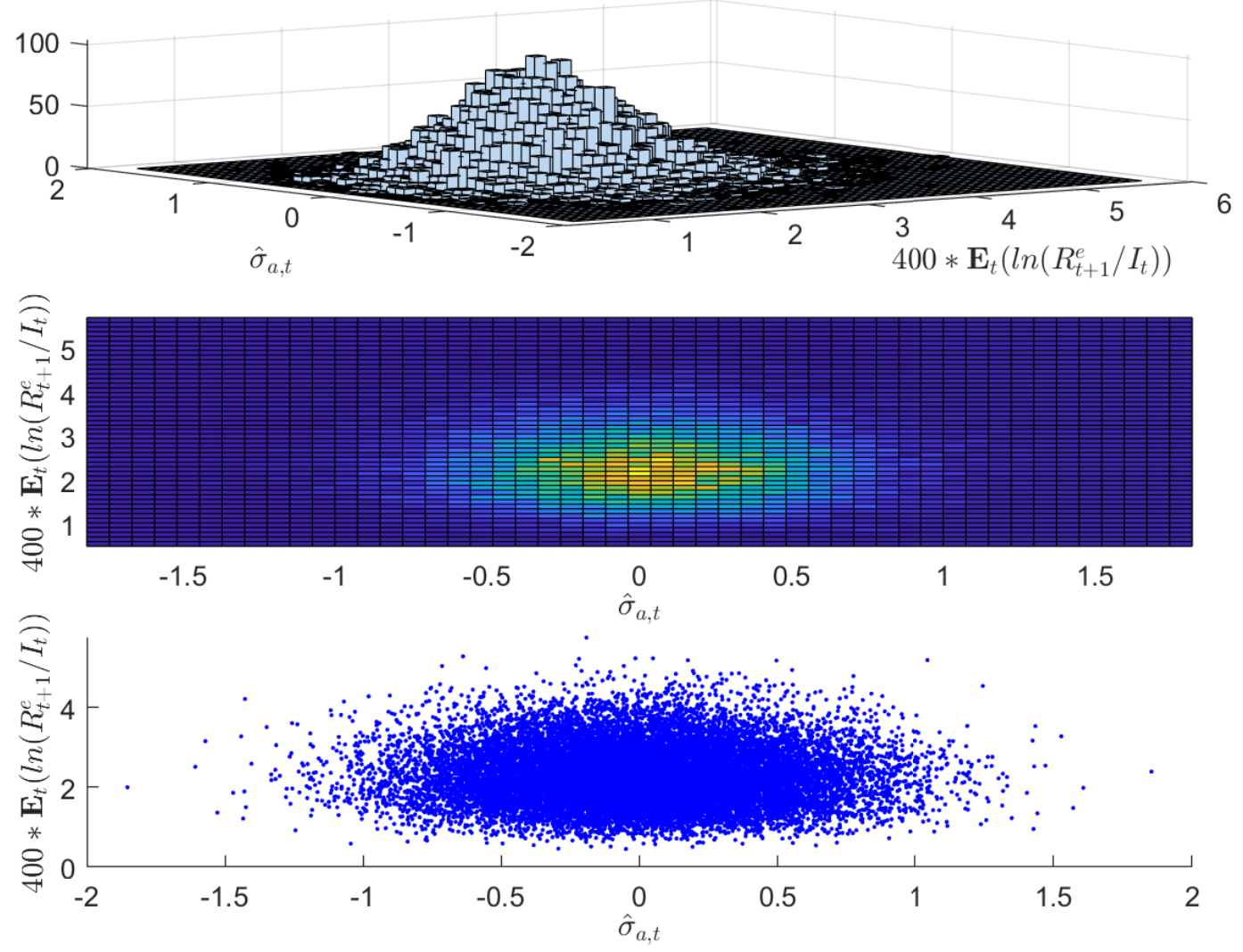
Figure 12D. Joint Distribution of the Endogenous Spread and Interest Rate Stochastic Volatility.

Joint distribution of $400 * \mathbf{E}_{t}\left(\ln \left(R_{t+1}^{e} / I_{t}\right)\right)$ and $\hat{\sigma}_{m, t}$
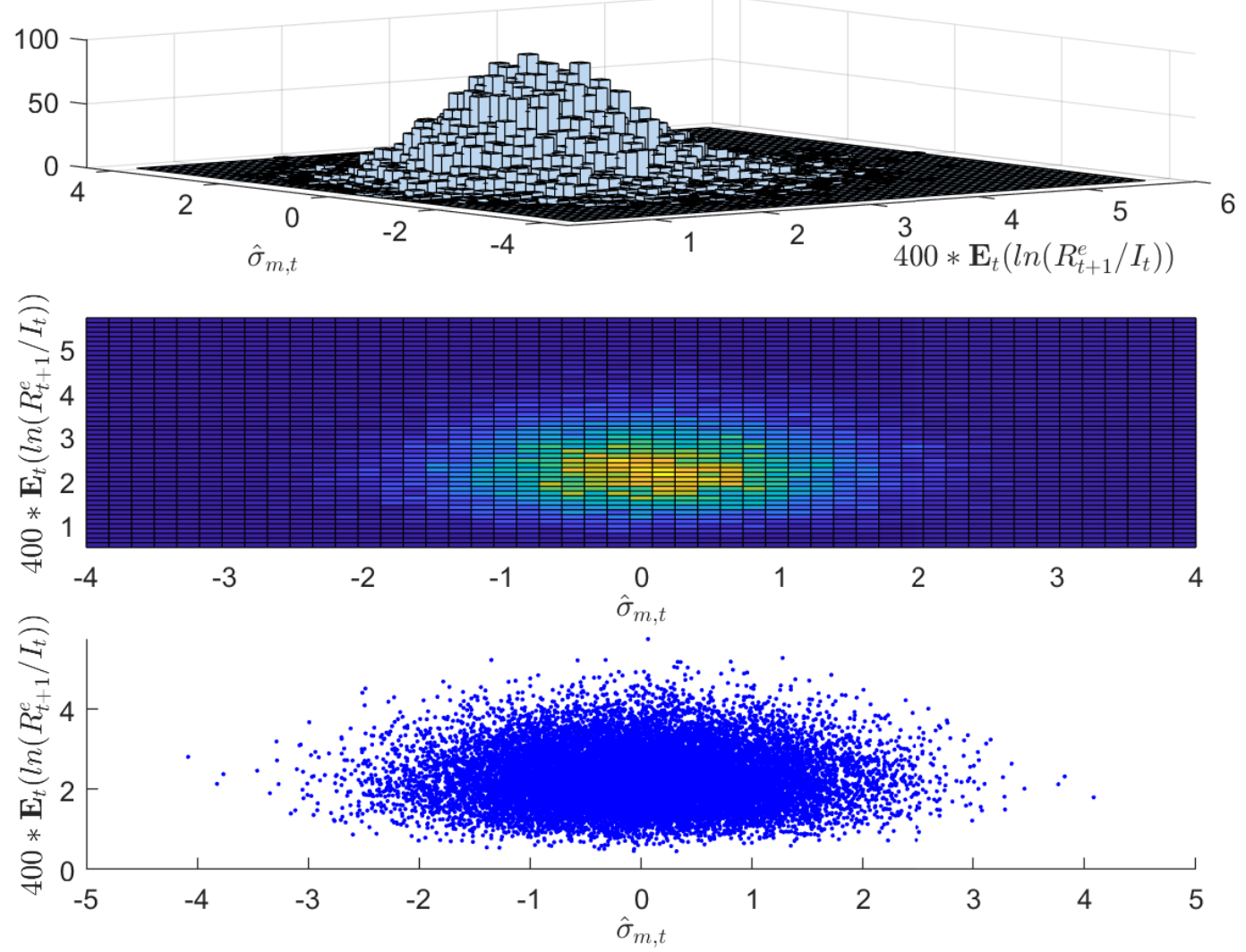
Figure 12E. Joint Distribution of the Endogenous Spread and Micro-Uncertainty.

Joint distribution of $400 * \mathbf{E}_{t}\left(\ln \left(R_{t+1}^{e} / I_{t}\right)\right)$ and $\hat{\sigma}_{\omega, t}$
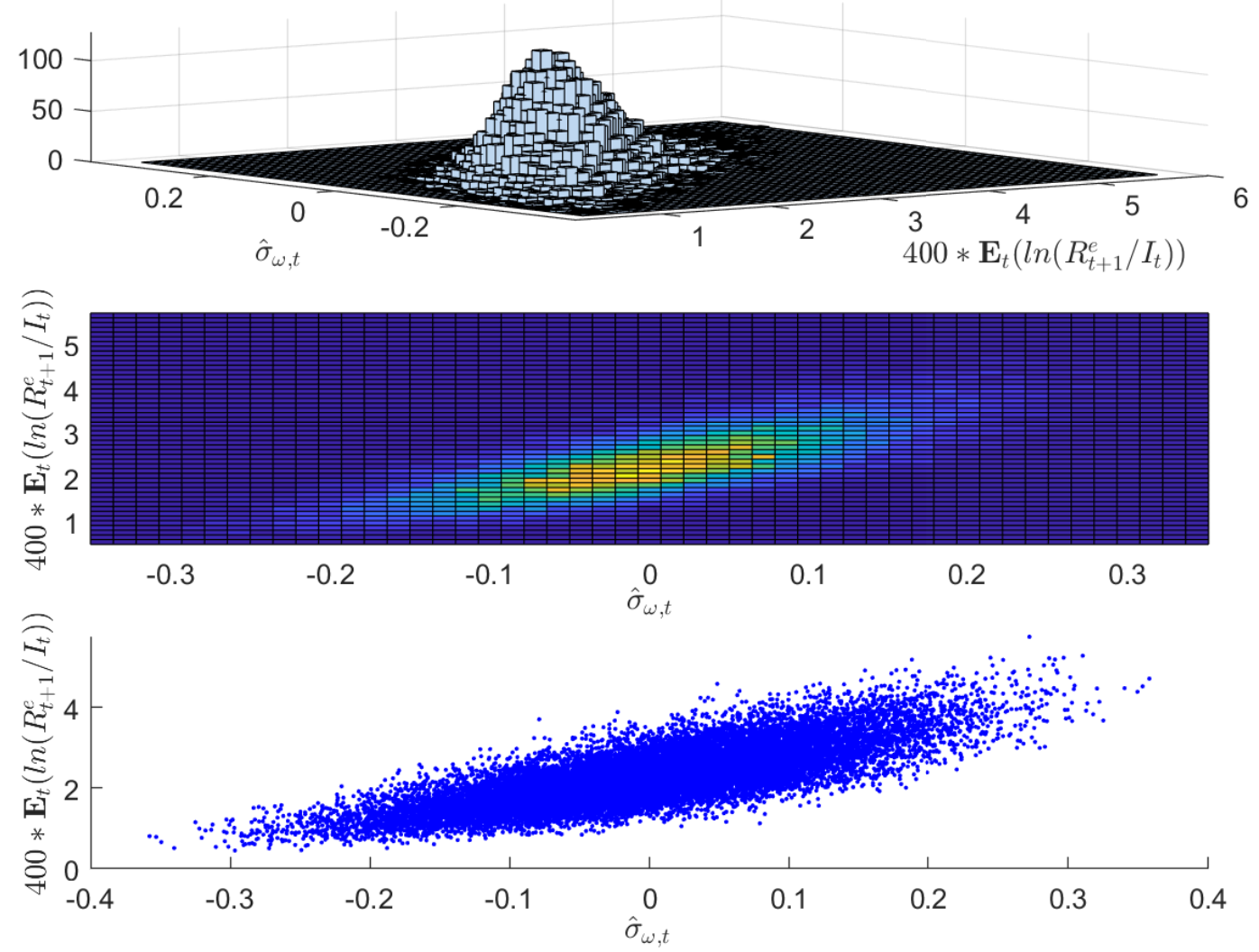
Figure 12F. Joint Distribution of the Endogenous Spread and Capital.

Joint distribution of $400 * \mathbf{E}_{t}\left(\ln \left(R_{t+1}^{e} / I_{t}\right)\right)$ and $400 * \ln \left(K_{t+1}\right)$
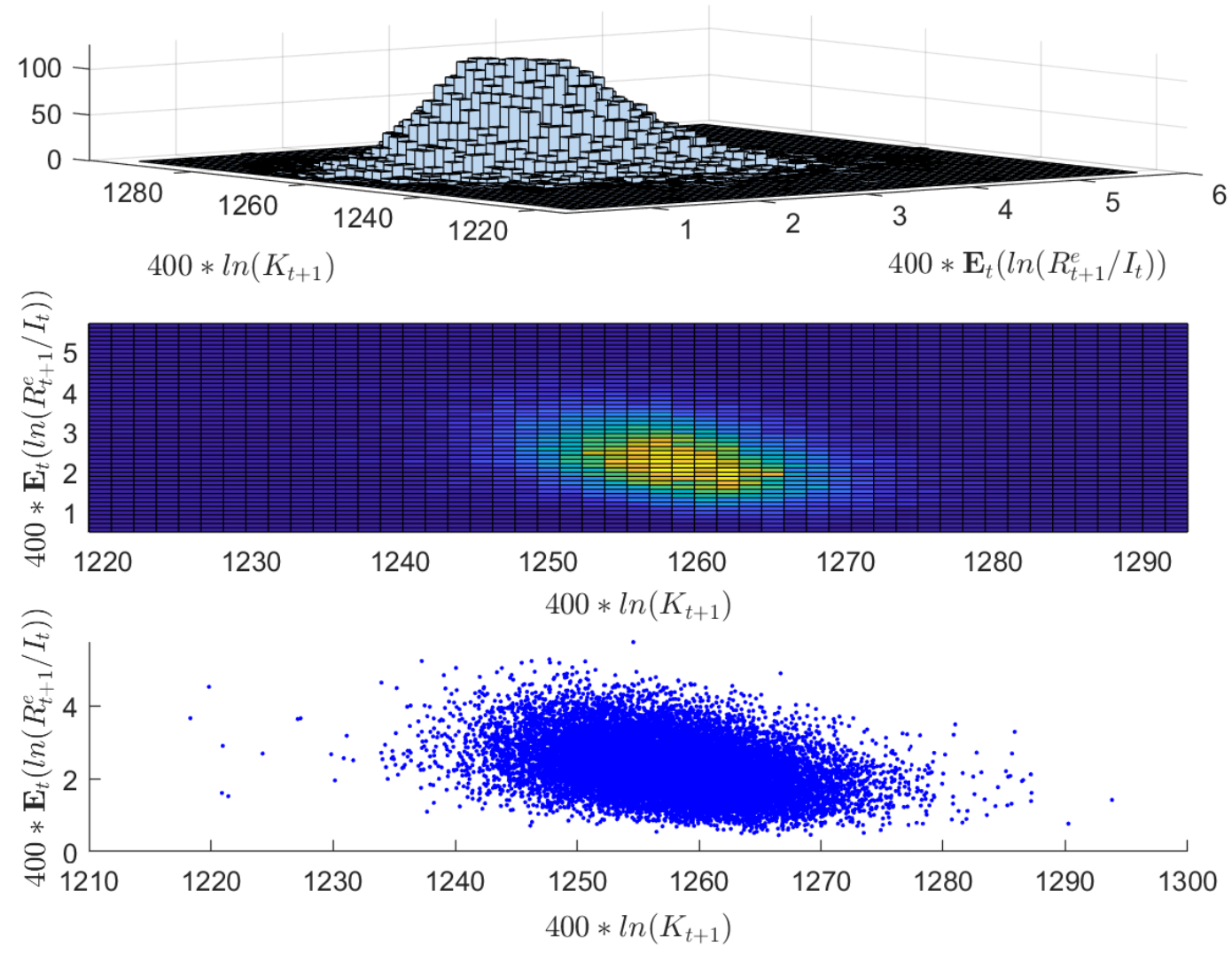
Figure 12G. Joint Distribution of the Endogenous Spread and Real Net Worth.

Joint distribution of $400 * \mathbf{E}_{t}\left(\ln \left(R_{t+1}^{e} / I_{t}\right)\right)$ and $400 * \ln \left(N_{t}^{r}\right)$
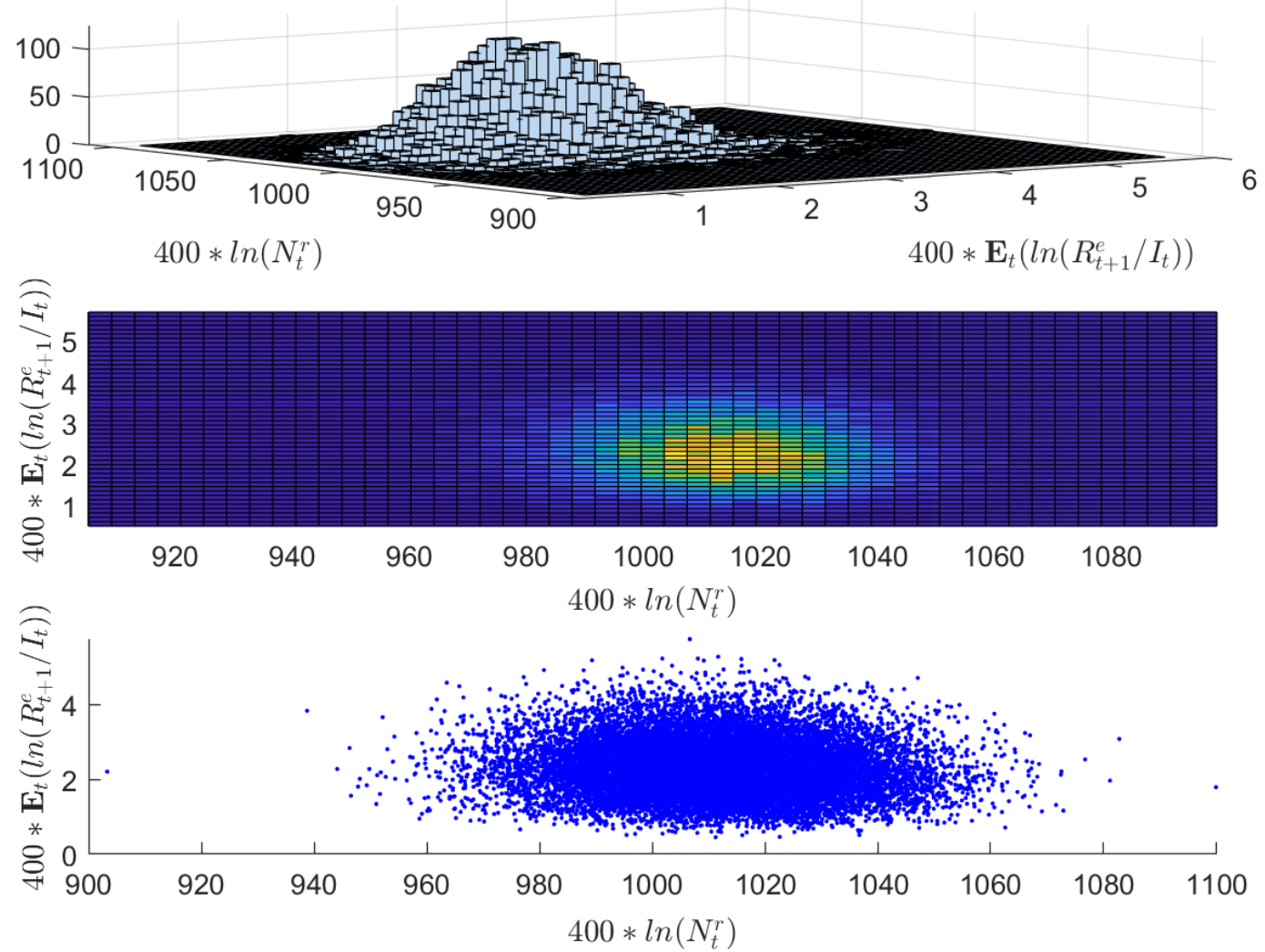
Figure 12H. Joint Distribution of the Endogenous Spread and Output.

Joint distribution of $400 * \mathbf{E}_{t}\left(\ln \left(R_{t+1}^{e} / I_{t}\right)\right)$ and $400 * \ln \left(Y_{t}\right)$
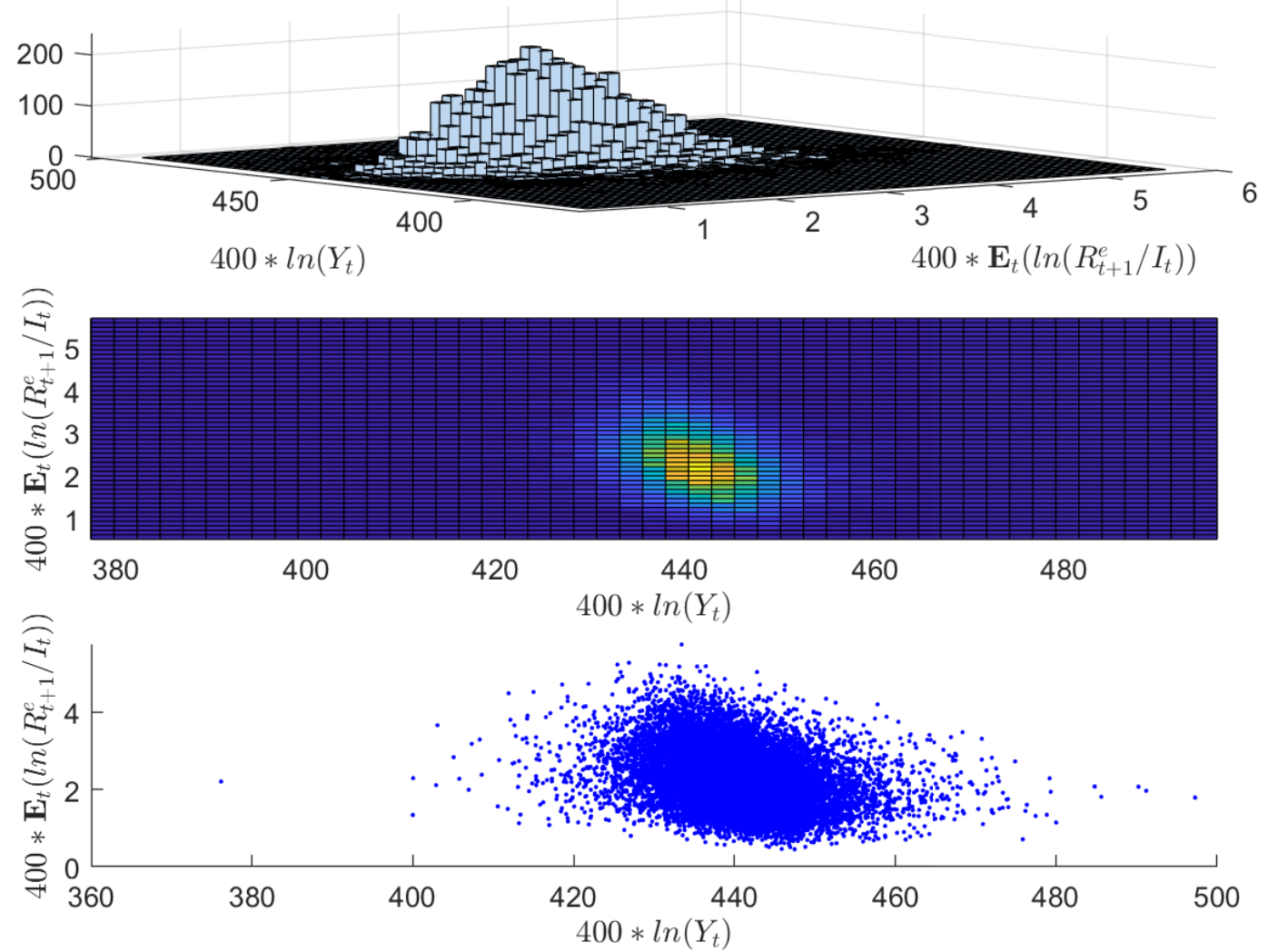
Figure 13A. Joint Distribution of the Endogenous Spread and TFP when Households' Inverse of the Intertemporal Elasticity of Substitution is Increased to $\chi=7$.
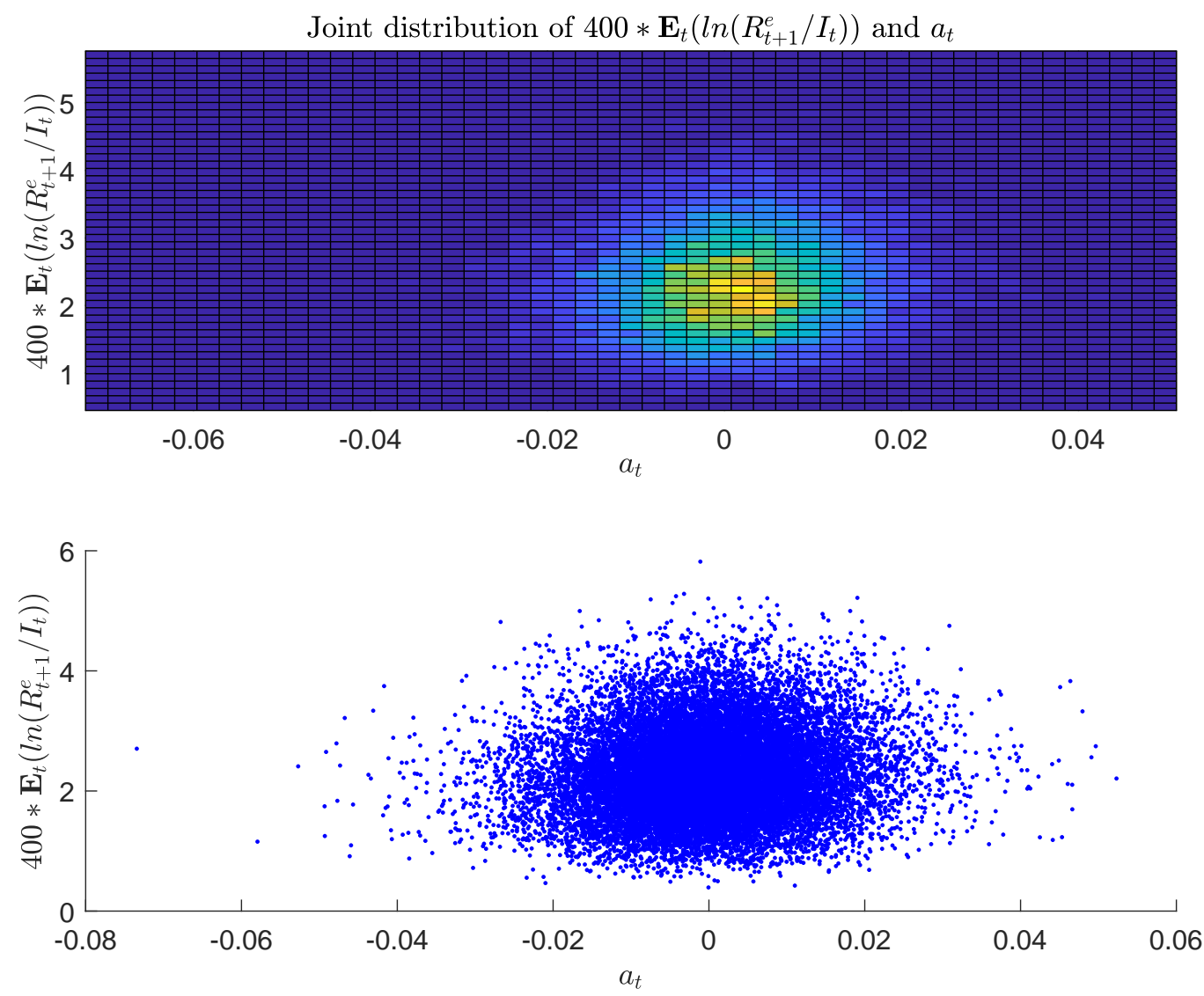
Figure 13B. Joint Distribution of the Endogenous Spread and Interest Rate when Households' Inverse of the Intertemporal Elasticity of Substitution is Increased to $\chi=7$.
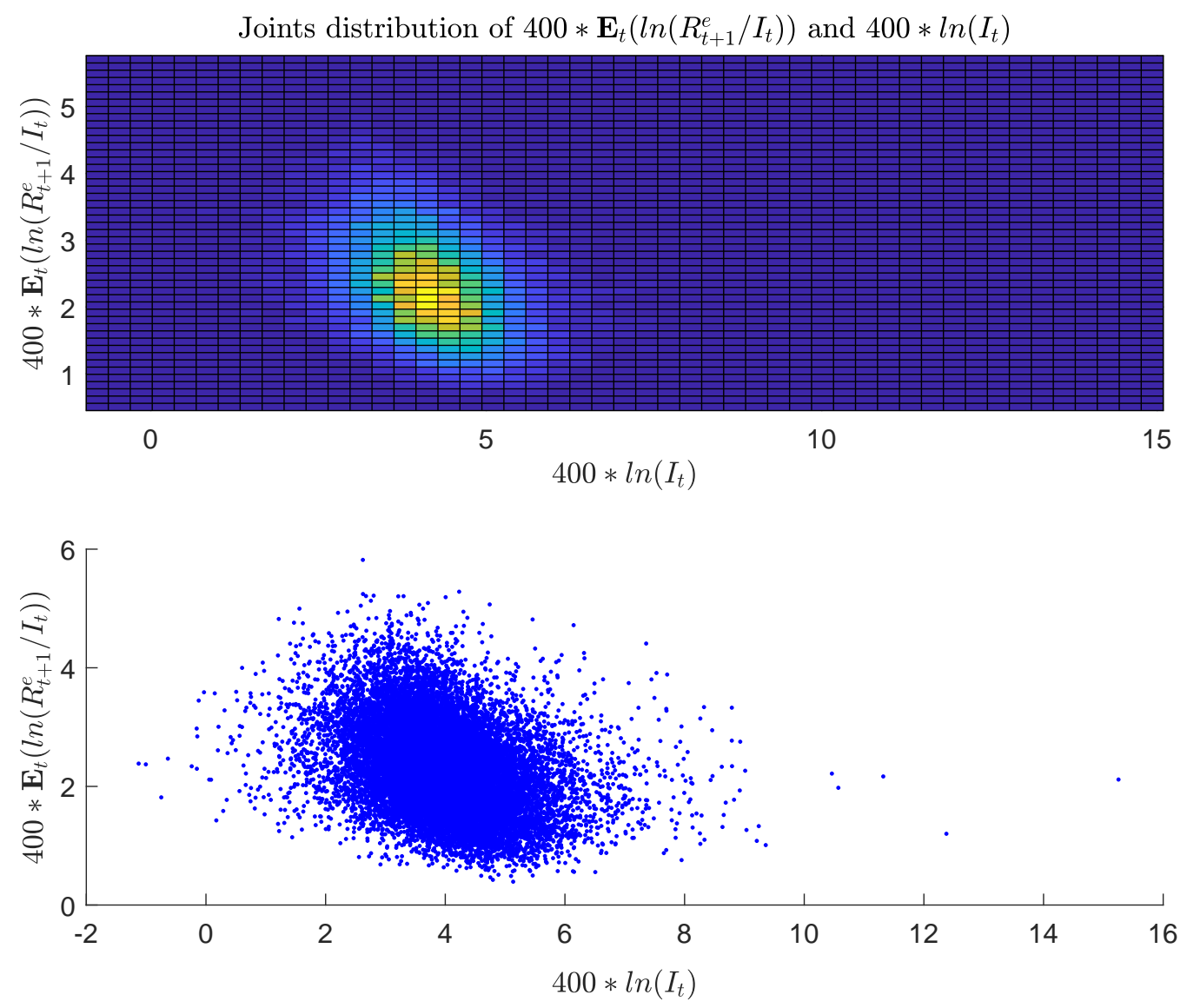
Figure 13C. Joint Distribution of the Endogenous Spread and TFP Stochastic Volatility when Households' Inverse of the Intertemporal Elasticity of Substitution is Increased to $\chi=7$.
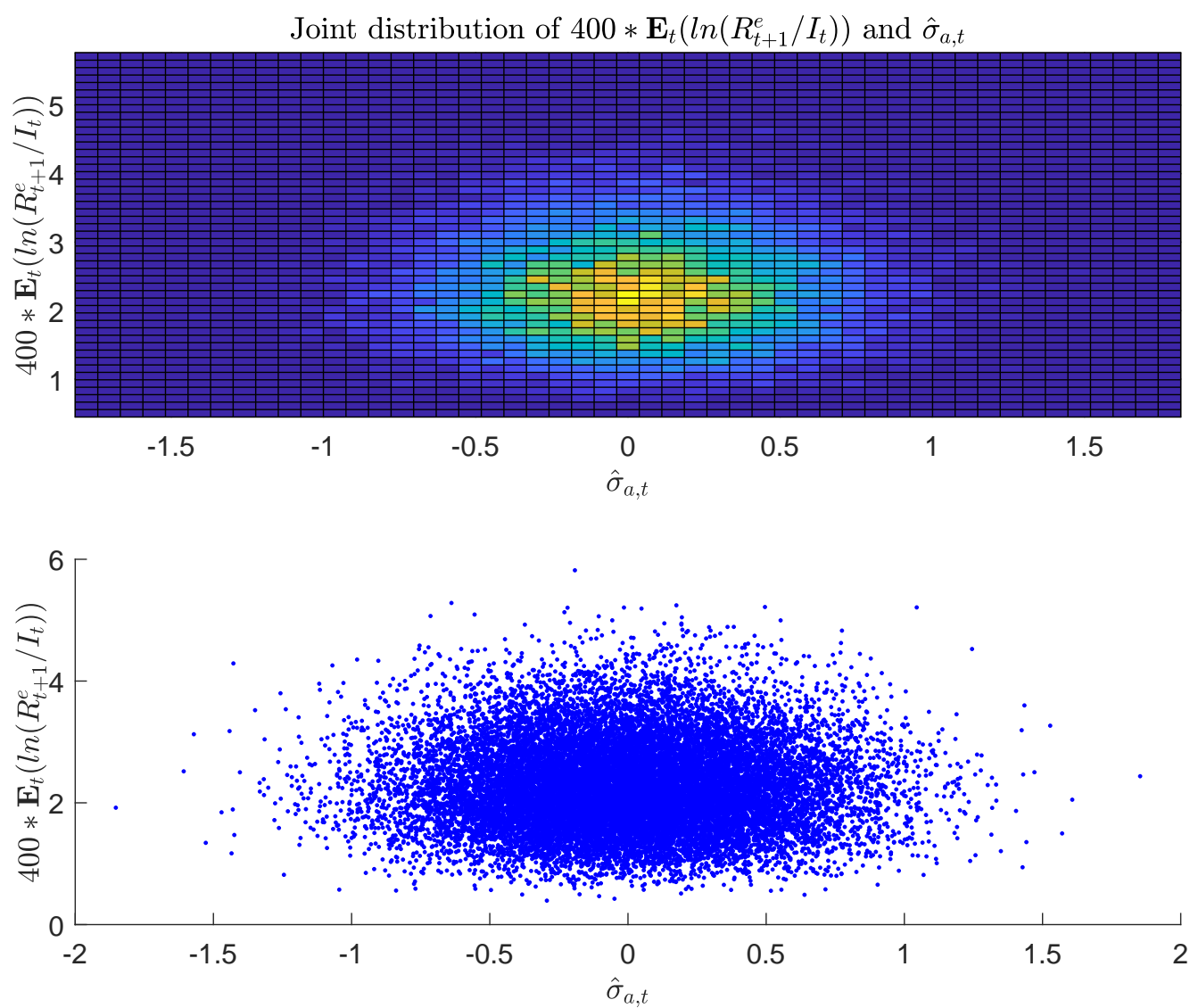
Figure 13D. Joint Distribution of the Endogenous Spread and Interest Rate Stochastic Volatility when Households' Inverse of the Intertemporal Elasticity of Substitution is Increased to $\chi=7$.
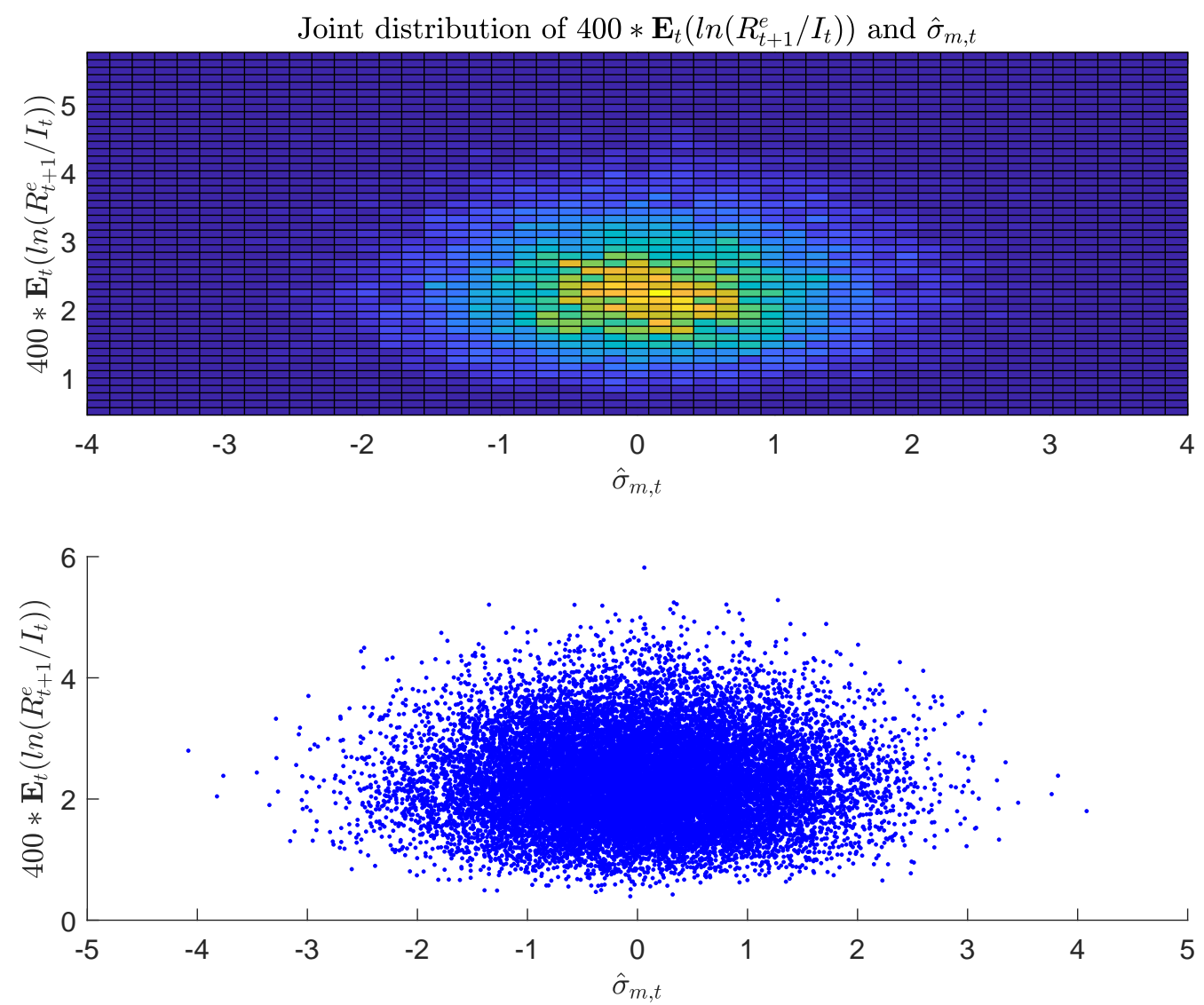
Figure 13E. Joint Distribution of the Endogenous Spread and Micro-Uncertainty when Households' Inverse of the Intertemporal Elasticity of Substitution is Increased to $\chi=7$.
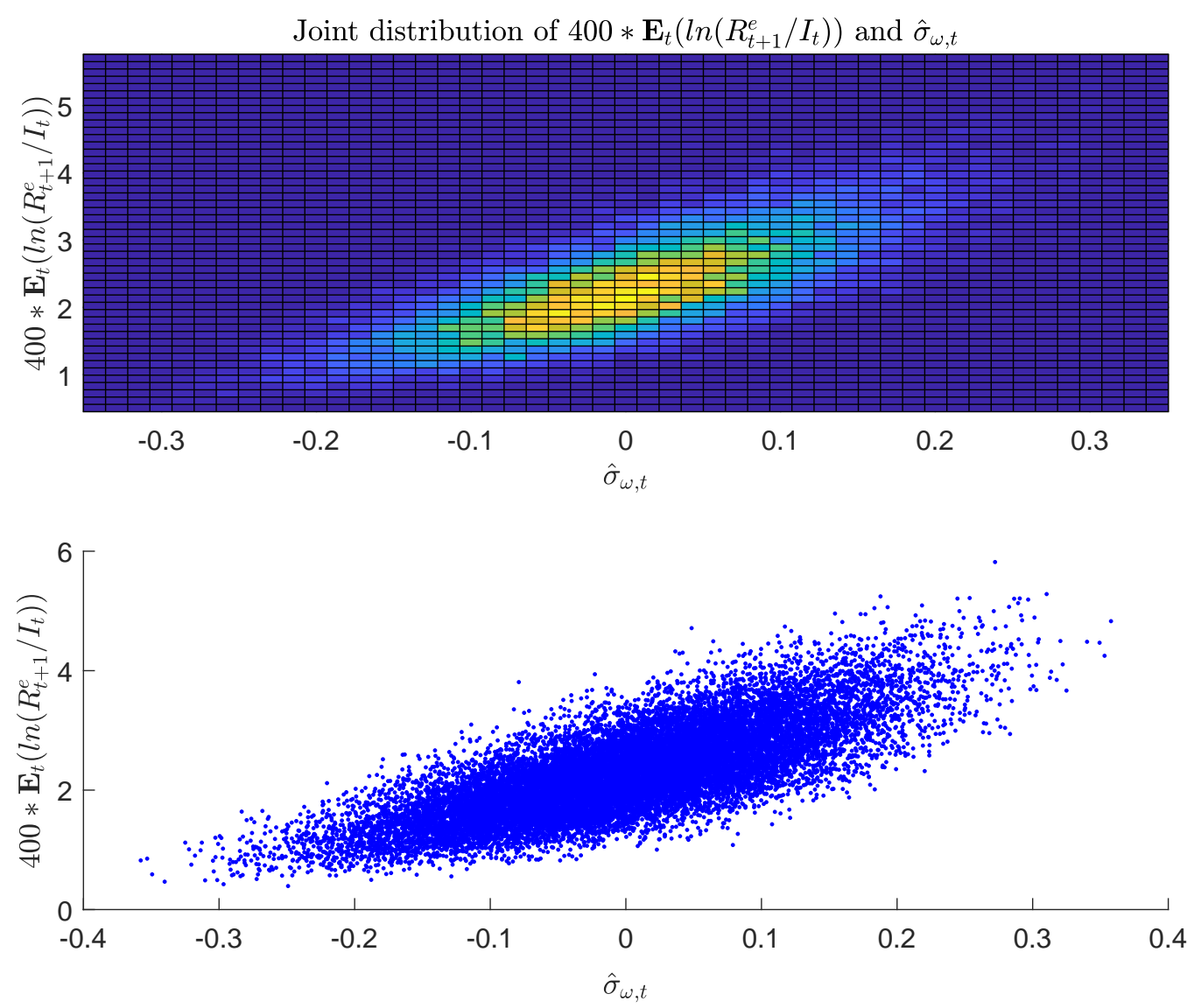
Figure 13F. Joint Distribution of the Endogenous Spread and Capital when Households' Inverse of the Intertemporal Elasticity of Substitution is Increased to $\chi=7$.
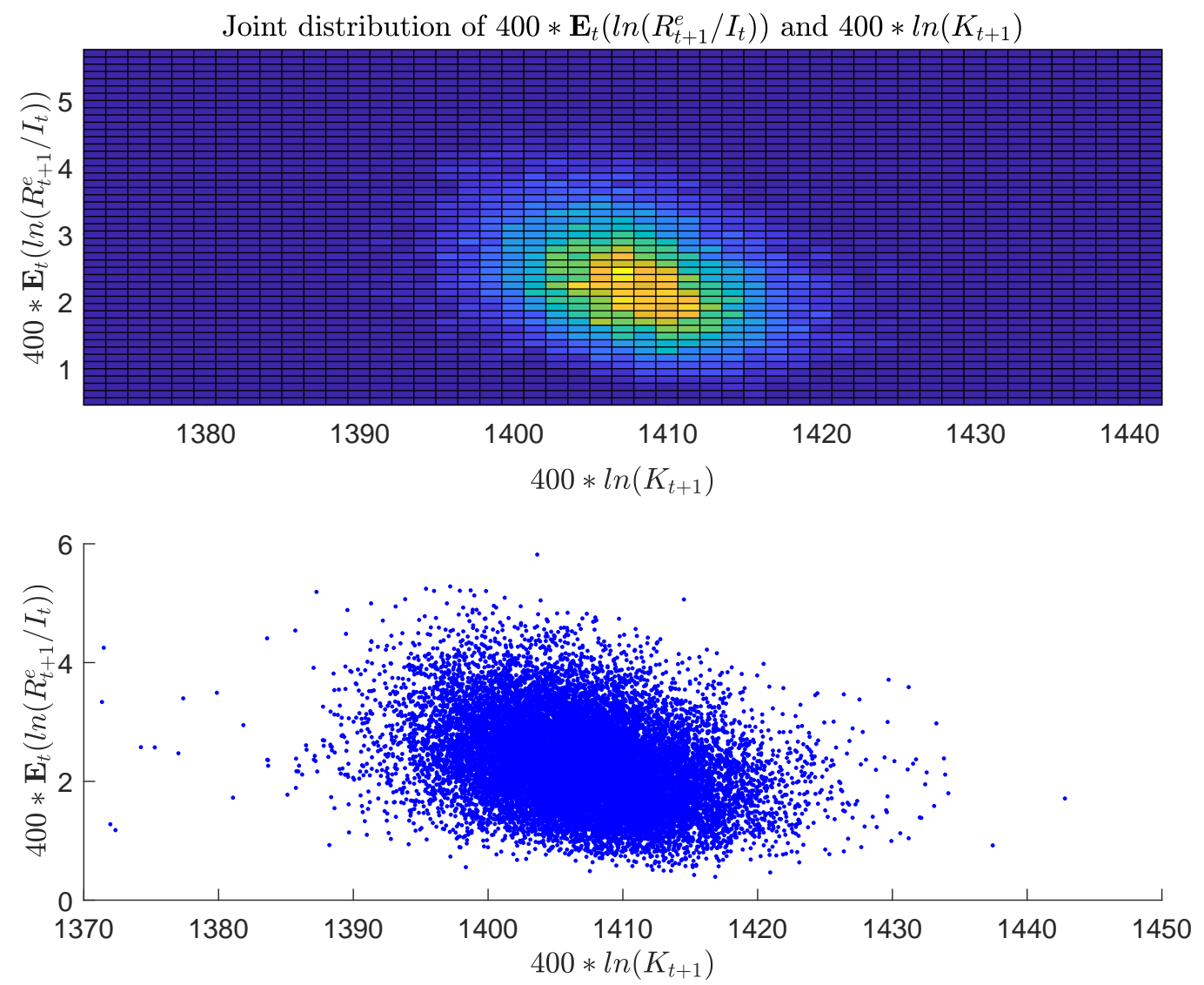
Figure 13G. Joint Distribution of the Endogenous Spread and Real Net Worth when Households' Inverse of the Intertemporal Elasticity of Substitution is Increased to $\chi=7$.
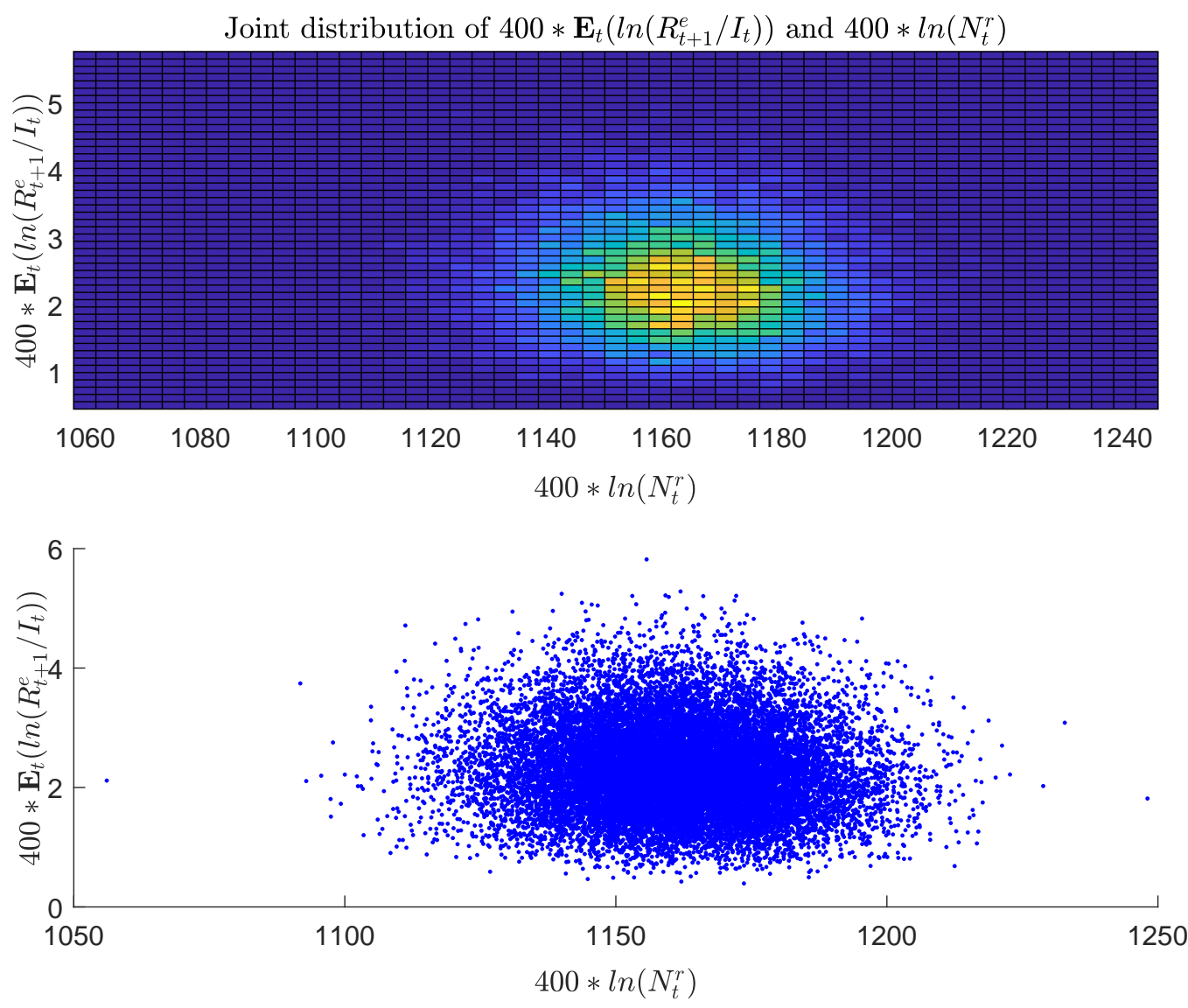
Figure 13H. Joint Distribution of the Endogenous Spread and Output when Households' Inverse of the Intertemporal Elasticity of Substitution is Increased to $\chi=7$.
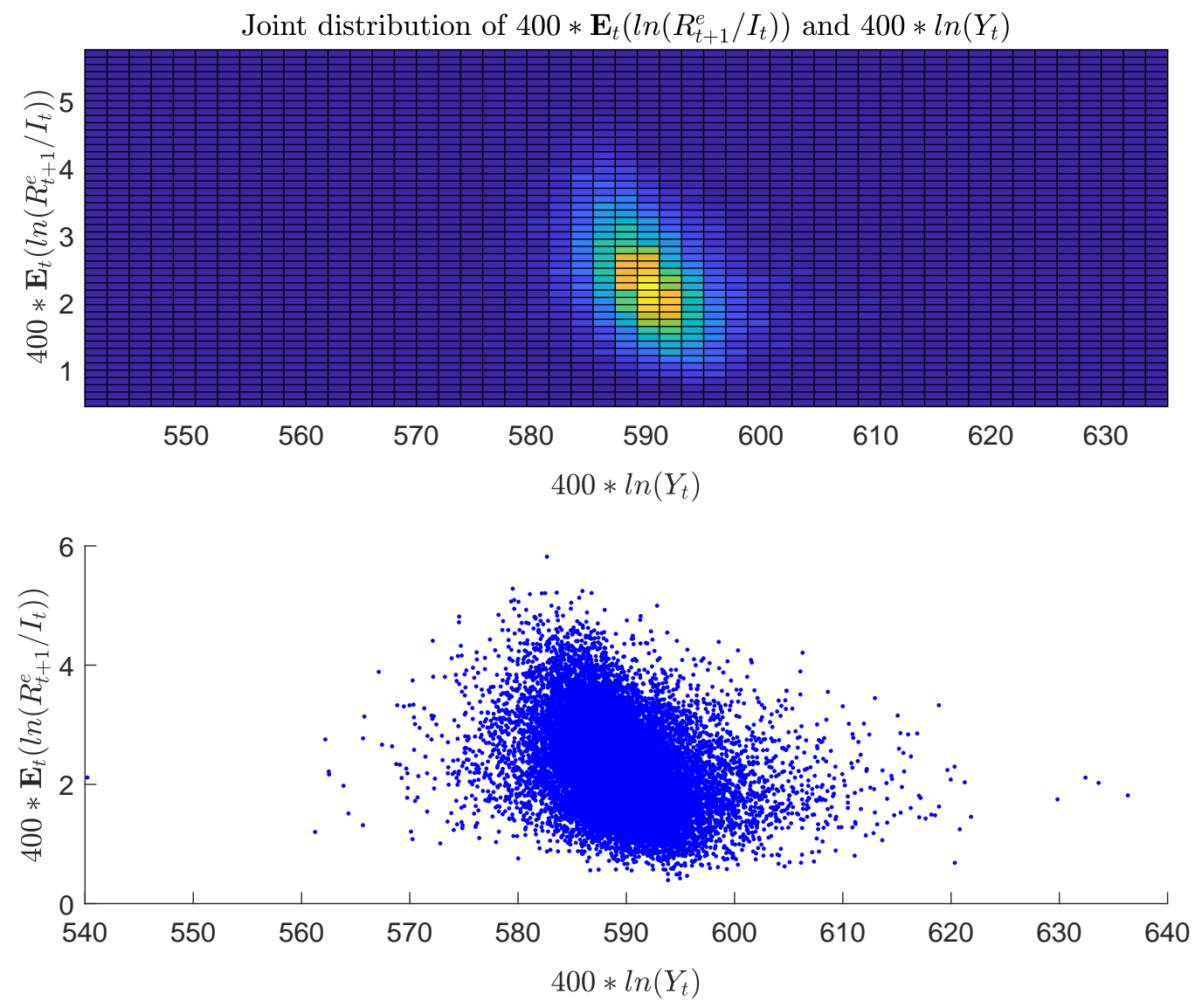


\section{Bibliography}

Aruoba, S. B., J. Fernández-Villaverde, and J. F. Rubio-Ramírez (2006). Comparing Solution Methods for Dynamic Equilibrium Economies. Journal of Economic Dynamics and Control 30(12), 2477-2508. https://doi.org/10.1016/j.jedc.2005.07.008.

Balke, N., E. Martínez-García, and Z. Zeng (2017). Understanding the Aggregate Effects of Credit Frictions and Uncertainty. Federal Reserve Bank of Dallas Globalization and Monetary Policy Institute Working Paper No. 317. June 2017 (Revised: October 2019). https://doi.org/10.24149/gwp317r1.

Basu, S. (1996). Procyclical Productivity: Increasing Returns or Cyclical Utilization? Quarterly Journal of Economics 111(3), 719-51. https://doi.org/10.2307/2946670.

Bernanke, B. S., M. L. Gertler, and S. Gilchrist (1999). The Financial Accelerator in a Quantitative Business Cycle Framework. In J. B. Taylor and M. Woodford (Eds.), Handbook of Macroeconomics, vol. 1, Chapter 21, pp. 1341-1393. Elsevier Science B.V. https://doi.org/10.1016/s1574-0048(99)10034-x.

Born, B. and J. Pfeifer (2014). Policy Risk and the Business Cycle. Journal of Monetary Economics 68(68-85). https://doi.org/10.1016/j.jmoneco.2014.07.012.

Carlstrom, C. T., T. S. Fuerst, and M. Paustian (2016). Optimal Contracts, Aggregate Risk, and the Financial Accelerator. American Economic Journal: Macroeconomics 8(1), 119-147. https://doi.org/10.1257/mac.20120024.

Cesa-Bianchi, A. and E. Fernández-Corugedo (2018). Uncertainty, Financial Frictions, and Nominal Rigidities: A Quantiative Investigation. Journal of Money, Credit and Banking 50(4), 603-636. https://doi.org/10.1111/jmcb.12505.

Christiano, L. J., M. Eichenbaum, and C. L. Evans (2005). Nominal Rigidities and the Dynamic Effects of a Shock to Monetary Policy. Journal of Political Economy 113(1), 1-45. https://doi.org/10.1086/426038.

Christiano, L. J., R. Motto, and M. Rostagno (2014). Risk Shocks. American Economic Review 104(1), 27-65. https://www.doi.org/10.1257/aer.104.1.27.

Dorofeenko, V., G. S. Lee, and K. D. Salyer (2008). Time-Varying Uncertainty and the Credit Channel. Bulletin of Economic Research 60(4), 375-403. https://doi.org/10.1111/j.1467-8586.2008.00284.x.

Fernández-Villaverde, J. (2010). The Econometrics of DSGE Models. SERIEs 1(1-2), 349. https://doi.org/10.1007/s13209-009-0014-7. 
Fernández-Villaverde, J., P. Guerrón-Quintana, and J. F. Rubio-Ramírez (2010). Fortune or Virtue: Time-Variant Volatilities Versus Parameter Drifting in U.S. Data. NBER Working Paper no. 15928. https://doi.org/10.3386/w15928.

Hellwig, M. F. (2001). Risk Aversion and Incentive Compatibility with Ex Post Information Asymmetry. Economic Theory 18(2), 415-438. https://doi.org/10.1007/PL00004192.

Kiyotaki, N. and J. Moore (1997). Credit Cycles. Journal of Political Economy 105(2), 211-248. https://doi.org/10.1086/262072.

Martínez-García, E. (2014). U.S. Business Cycles, Monetary Policy and the External Finance Premium. In F. Schleer-van Gellecom (Ed.), Advances in Non-Linear Economic Modeling: Theory and Applications, Volume 17 of Dynamic Modeling and Econometrics in Economics and Finance, Chapter 2, pp. 41-114. Berlin, Heidelberg: Spriger.

Martínez-García, E. (2018). Finite-Order VAR Representation of Linear Rational Expectations Models: With Some Lessons for Monetary Policy. Federal Reserve Bank of Dallas, Globalization and Monetary Policy Institute Working Paper no. 285. September 2016 (Revised: August 2018). https://doi.org/10.24149/gwp285r2.

Mehra, Y. (2004). The Output Gap, Expected Future Inflation and Inflation Dynamics: Another Look. The B.E. Journal of Macroeconomics 4(1), 1-19. https://doi.org/10.2202/1534-5998.1194.

Monnet, C. and E. Quintin (2005). Optimal Contracts in a Dynamic Costly State Verification Model. Economic Theory 26(4), 867-885. https://doi.org/10.1007/s00199-0040535-z.

Rotemberg, J. J. (1982). Sticky Prices in the United States. Journal of Political Economy $90(6)$, 1187-1211. https://www.jstor.org/stable/1830944.

Schmitt-Grohé, S. and M. Uribe (2004). Solving Dynamic General Equilibrium Models Using a Second-Order Approximation to the Policy Function. Journal of Economic Dynamics and Control 28(4), 755-775. https://doi.org/10.1016/S0165-1889(03)000435.

Smets, F. and R. Wouters (2007). Shocks and Frictions in U.S. Business Cycles: A Bayesian DSGE Approach. American Economic Review 97(3), 586-606. https://doi.org/10.1257/aer.97.3.586. 
Stock, J. H. and M. W. Watson (1999). Forecasting Inflation. Journal of Monetary Economics 44(2), 293-335. https://doi.org/10.1016/S0304-3932(99)00027-6.

Taylor, J. B. (1993). Discretion versus Policy Rules in Practice. Carnegie-Rochester Conference Series on Public Policy 39, 195-214. https://doi.org/10.1016/01672231(93)90009-1. 